\title{
POLITÉCNICA
}

\author{
Universidad Politécnica de Madrid
}

Escuela Técnica Superior de Ingenieros Informáticos

\section{ANÁLISIS Y GESTIÓN DEL RIESGO EN LOS SISTEMAS DE INFORMACIÓN: UN ENFOQUE BORROSO}

\section{TESIS DOCTORAL}

\section{ELOY VICENTE CESTERO}

Licenciado en Matemáticas 



\section{Universidad Politécnica de Madrid}

Escuela Técnica Superior de Ingenieros Informáticos

Departamento de Inteligencia Artificial

\section{ANÁLISIS Y GESTIÓN DEL RIESGO EN LOS SISTEMAS DE INFORMACIÓN: UN ENFOQUE BORROSO}

Autor: Eloy Vicente Cestero

Licenciado en Matemáticas

Directores: D. Alfonso Mateos Caballero Licenciado en CC. Matemáticas y Doctor en Informática

D. Antonio Jiménez Martín

Licenciado en Informática y Doctor en Informática

Septiembre, 2016 



$$
e^{\pi i}=-1
$$

...Esa bella dama que sólo enseña sus encantos a quien porfía con ella... No queda sino batirse...

A mis padres.

A Susana. 



\section{Agradecimientos}

En primer lugar, agradecer a los profesores Alfonso Mateos Caballero y Antonio Jiménez Martín las enseñanzas y ayudas recibidas y el compañerismo mostrado en los diferentes proyectos de investigación que hemos compartido.

A toda mi familia, y en particular a mis padres, por la ayuda y la comprensión recibidas a lo largo de mi amplia etapa académica. No fue fácil pero tampoco desistimos.

A Susana por compartir conmigo este esfuerzo y por hacerme feliz.

A mi compañero de máster y doctorado Italo Farfán Vera con quien espero mantener siempre una buena relación de camaradería.

El desarrollo de esta tesis ha sido posible gracias a la financiación de los proyectos de investigación S2009/ESP-1685 de la Comunidad de Madrid, MTM2011-28983-C03-03 del Ministerio de Ciencia e Innovación y MTM2014-56949-C3-2R del Ministerio de Economía y Competitividad. 



\section{Resumen}

Las metodologías oficiales de análisis y gestión de riesgos en sistemas de información (SI), reguladas por las normativas internacionales de la serie ISO/IEC 27000, sugieren definir relaciones entre los activos del sistema, de modo que el ataque sobre uno de ellos se puede transmitir con cierta probabilidad a lo largo de toda la red, llegando a alcanzar a los activos más valiosos para la organización. De este modo, los activos de un SI constituyen los nodos de un grafo dirigido y acíclico en el que cada arco está ponderado por la probabilidad de transmisión de fallos entre dos activos.

Por otro lado, deben considerarse el valor de los activos, la probabilidad de materialización de una amenaza y la degradación que ésta puede provocar sobre los activos, y deben establecerse indicadores de impacto y riesgo a partir de estas variables.

Sin embargo, los expertos encargados de asignar tales valores a menudo aportan únicamente información imprecisa, de modo que la Lógica Borrosa puede ser muy útil en este ámbito. Es necesario facilitar a los expertos un método con el que puedan expresar de forma imprecisa sus juicios probabilísticos o sus valoraciones sobre los activos, y una vez obtenidos tales valores, hemos de construir algoritmos que nos permitan establecer, bajo este tratamiento borroso, los indicadores de impacto y riesgo para las amenazas que se ciernen sobre los activos de información.

Finalmente, debemos encontrar un conjunto óptimo de salvaguardas que evite la materialización de una amenaza o disminuya la degradación provocada sobre los activos, reduciendo así el riesgo asociado al sistema.

En esta tesis doctoral proponemos un enfoque borroso del análisis y gestión de riesgos en los SI que toma como base la metodología MAGERIT, establecida para adoptar las normas ISO/IEC 27000 en nuestro país. 



\section{Abstract}

Official information system (IS) risk analysis and management methodologies, regulated by the ISO/IEC family of international standards suggest defining relationships between system assets. Consequently, an attack on one asset has a certain probability of being propagated throughout the network and reaching the organization's most valuable assets. Thus, IS assets are the nodes of a directed and acyclic graph in which each edge is weighted by the probability of transmission between two assets.

On the other hand, asset values, the threat probabilities and potential asset degradation, and impact and risk indicators should be established based on these variables.

However, the experts responsible for assigning these values often provide only vague information. Fuzzy Logic can be very useful in this situation. Experts have need of a method to express their probabilistic judgments or asset valuations. Algorithms then have to be built that can use the resulting values to establish impact and risk indicators for potential threats to assets within this fuzzy environment.

Finally, we have identified an optimal set of safeguards to prevent the materialization of a threat or reduce the resulting asset degradation, thereby reducing the risk associated with the system.

In this $\mathrm{PhD}$ thesis we propose a fuzzy IS risk analysis and management approach that considers all these elements based on ISO / IEC 27000 standards, and particularly the Spanish MAGERIT methodology which is compliant international standards. 



\section{Índice general}

1. Introducción $\quad 1$

2. Objetivos 5

3. Elementos de lógica borrosa en el modelado de la incertidumbre $\quad 7$

3.1. Introducción . . . . . . . . . . . . . . . . . 7

3.2. Números borrosos. Aritmética y topología . . . . . . . . . . . . . 11

3.3. Modelado lingüístico borroso . . . . . . . . . . . . . . 16

3.4. El modelo computacional y la función de similitud . . . . . . . . . . . . 19

3.5. Educción de probabilidades mediante números borrosos . . . . . . . . 20

3.5.1. De la teoría clásica de la probabilidad a la teoría de la probabilidad lingüística . . . . . . . . . . . . . . . 21

3.5.2. Asignación de probabilidades mediante apuestas y loterías . . . . . 24

3.5.3. Asignación de probabilidades borrosas mediante apuestas y loterías 29

3.5.4. Calidad de la información. Medidas de borrosidad y de consistencia

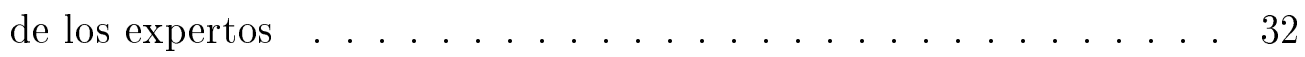

3.6. Un experimento computacional . . . . . . . . . . . . . 37

3.6.1. Modelización de la importancia de la experiencia del experto . . . . 38

3.6.2. Simulación de diálogos analista-experto . . . . . . . . . . . 39

3.6.3. Experimento . . . . . . . . . . . . . . . . 41

4. Similitud de números borrosos trapezoidales generalizados $\quad 51$

4.1. Funciones de similitud de números borrosos trapezoidales . . . . . . . . . 52

4.2. Similitud de consenso . . . . . . . . . . . . . 57 
4.2.1. Función de similitud de consenso . . . . . . . . . . . . . 60

4.2.2. Ventajas de la similitud de consenso . . . . . . . . . . . . . . 72

5. Metodologías de análisis y gestión de riesgos en los sistemas de información $\quad 73$

5.1. El ciclo de mejora continua . . . . . . . . . . . . . . . . . . . 74

5.2. La serie ISO $/$ IEC $27000 \ldots \ldots \ldots \ldots \ldots$

5.3. Términos y definiciones . . . . . . . . . . . . . . . 79

5.4. La norma ISO $/$ IEC $27001 . \ldots \ldots \ldots \ldots$. . . . . . . . . 80

5.5. Análisis y gestión de riesgos en los sistemas de gestión de seguridad de la información. La norma ISO/IEC $27005 \ldots$. . . . . . . . . . . 86

5.5.1. Identificación y valoración de los riesgos . . . . . . . . . . 87

5.5.2. Tratamiento de los riesgos . . . . . . . . . . . . . 92

6. Análisis y gestión borrosa de riesgos en los sistemas de información 95

6.1. Análisis y gestión de riesgos basado en la metodología MAGERIT . . . . . 96

6.2. Borrosificación del análisis de riesgos basado en la metodología MAGERIT 101

7. Selección de salvaguardas para la gestión borrosa del riesgo mediante $\begin{array}{ll}\text { metaheurísticas y programación dinámica } & 109\end{array}$

7.1. Introducción . . . . . . . . . . . . . . . . . . . . . 109

7.1.1. Recocido simulado . . . . . . . . . . . . . . . 114

7.1.2. Programación dinámica . . . . . . . . . . . . . 116

7.2. Tipos de salvaguardas y su efecto sobre el riesgo . . . . . . . . . . . . 121

7.3. Minimización de la probabilidad de transmisión de fallos . . . . . . . . . 122

7.4. Selección óptima de salvaguardas en la gestión de riesgos . . . . . . . . . 131

8. Un ejemplo ilustrativo $\quad 139$

8.1. Introducción . . . . . . . . . . . . . . . . . 139

8.2. Análisis de riesgos . . . . . . . . . . . . . . 140

8.2.1. Servicio de tramitación . . . . . . . . . . . . . . . 140

8.2.2. Servicio de archivo central . . . . . . . . . . . . . . 141

8.2.3. Equipamiento informático . . . . . . . . . . . . . . . 141 
8.2.4. Comunicaciones . . . . . . . . . . . . . . . . 142

8.2.5. Seguridad física . . . . . . . . . . . . . . . . 142

8.2.6. Análisis borroso . . . . . . . . . . . . . . . 143

8.3. Selección de salvaguardas . . . . . . . . . . . . . . . . 147

9. Conclusiones y líneas futuras de investigación $\quad 155$

9.1. Líneas futuras de investigación . . . . . . . . . . . . . . . . . . 159 


\section{Capítulo 1 INTRODUCCIÓN}

En multitud de situaciones el enfoque subjetivo es el único enfoque posible para la asignación de probabilidades de ocurrencia de sucesos para los cuales no se tienen datos históricos previos, ni existe la posibilidad de poner en práctica mecanismos empíricos para la obtención de datos. Consideraciones de tipo ético, legal, técnico o económico impiden la elaboración de tales experimentos. De este modo, la Estadística Bayesiana se erige en el paradigma fundamental de asignación de probabilidades subjetivas, ya que permite la actualización de las probabilidades conforme los expertos van adquiriendo información sobre el suceso. Por esta razón, dicho paradigma es el más utilizado en los sistemas de ayuda a la decisión que necesitan valoraciones de expertos, como puede ser el análisis de riesgos en cualquier ámbito.

Sin embargo, en multitud de ocasiones el ambiente en el que se desarrolla un sistema de ayuda a la decisión es de incertidumbre, imprecisión y vaguedad, de modo que expertos y decisores son incapaces de dar valores concretos a las utilidades de las alternativas entre las cuales decidir, a los pesos de los distintos criterios de decisión, o a las probabilidades de ocurrencia de los distintos escenarios.

La Lógica Borrosa, creada por Lotfy A. Zadeh a mediados de la década de los años 60 del siglo pasado, es un paradigma de representación del conocimiento donde los objetos no tienen por qué "ser" o "no ser" elementos de un determinado conjunto, sino que puede haber matices continuos entre ambas condiciones. Dicho paradigma nos permite modelar términos lingüísticos vagos e imprecisos, del tipo "mucho", "mediano" o "poco", y operar con ellos en un proceso de inferencia.

Por ejemplo, cuando los expertos son incapaces de decidirse por un valor concreto al 
asignar probabilidades subjetivas a un suceso, puede ser útil, y de hecho es muy común, establecer escalas de términos lingüísticos como "muy bajo", "bajo", "medio", "alto", "muy alto" para asignar probabilidades sin exigir un compromiso excesivo del experto en cuanto a la precisión de sus juicios. Estos términos lingüísticos se pueden asociar a números borrosos trapezoidales normalizados con soporte en [0,1] para poder operar con ellos dentro de una aritmética adecuada. También existen métodos eficientes para extraer el juicio probabilístico borroso de los expertos, evitando cometer sesgos derivados del uso de escalas rígidas que centran la atención de los expertos en unos cuantos números borrosos trapezoidales.

En las metodologías estatales de análisis y gestión de riesgos en los Sistemas de Información (SI), tales como la norteamericana NIST [45], la francesa MEHARI [41] o la española MAGERIT [37, 38, 39], que toman como base la normas internacionales de la serie ISO/IEC 27000, se propone habitualmente la adopción de un modelo cuantitativo, en el que probabilidades y valoraciones son números reales; o un modelo cualitativo, en que se asigna un número natural a una escala de términos lingüísticos para dar probabilidades o valoraciones.

Los inconvenientes de ambos métodos son claros: El modelo cuantitativo asume que tenemos conocimientos precisos de la probabilidad de ocurrencia de todos los eventos y del valor de todos los activos, lo cual en la mayoría de los casos no es cierto. Por otro lado, el modelo cualitativo no justifica la asignación de un número natural a un término lingüístico, sino que más bien esta asignación obedece a cuestiones operativas, transformando información imprecisa suministrada por el experto en información nítida de manera arbitraria, únicamente manteniendo el orden natural de la escala lingüística.

A medio camino entre ambos enfoques, cualitativo y cuantitativo, se posiciona el enfoque borroso, que transforma la escala lingüística en números borrosos de modo que se mantiene la imprecisión suministrada por el experto y el orden de la escala lingüística, permitiendo a su vez, el uso de aritméticas borrosas adecuadas para operar con tales números. Por tanto, este enfoque recoge las ventajas de los modelos actuales promovidos por las metodologías estatales de análisis y gestión de riesgos en los SI, y evita los inconvenientes de cada uno de ellos.

Como hemos indicado anteriormente, la información lingüística suministrada por los 
expertos puede ser representada con números borrosos trapezoidales con los que se puede operar por medio de una aritmética adecuada o, en el caso de sistemas de control borrosos, son las entradas de un sistema basado en reglas de tipo IF/THEN. Pues bien, tanto los resultados de las aritméticas borrosas, como los de las reglas de inferencia, no son, en general, números borrosos de las escalas previamente definidas, de modo que para informar convenientemente de tales resultados, hemos de transportarlos a dichas escalas, y esto se hace por medio de funciones de similitud de números trapezoidales, de modo que el término lingüístico que se elige para informar de un resultado borroso es el término de la escala representado por el número borroso trapezoidal más parecido al resultado.

Para establecer la similitud entre los números borrosos implicados se puede recurrir a diferentes parámetros que dan una idea de la distancia entre ambos, de la forma o del tamaño. Estos parámetros se agregan en expresiones que definen el grado de similitud entre dos números borrosos. Sin embargo, todas las medidas de similitud tienen inconvenientes, debido a que los parámetros utilizados para definirlas no siempre son los más adecuados según las circunstancias del problema y de los números que el modelo maneja.

En esta tesis desarrollamos un nuevo enfoque para el análisis de riesgos en los sistemas de información basado en la lógica borrosa, proponiendo un algoritmo interactivo de educción del juicio probabilístico borroso del experto, una aritmética de números borrosos trapezoidales adecuada que, junto con un algoritmo recursivo, simplifica y mejora el modelo propuesto por las metodologías estatales consultadas, una nueva función de similitud de números borrosos trapezoidales que mejora al resto de funciones de la literatura, y un modelo de optimización en la selección de salvaguardas y controles en la gestión del riesgo en los SI mediante metaheurísticas y programación dinámica.

En el Capítulo 2 de esta tesis enumeramos los objetivos de la misma. En el Capítulo 3, exponemos los elementos de lógica borrosa que vamos a necesitar a lo largo de toda la tesis y proponemos un método de educción de probabilidades borrosas. En el Capítulo 4 realizamos un estado del arte sobre funciones de similitud de números borrosos trapezoidales, evaluando cada función en cuanto a sus ventajas e inconvenientes. También proponemos una función que mejora a las funciones encontradas en la literatura, y realizamos una comparativa.

En el Capítulo 5 se proporciona información sobre las normas internacionales relativas 
al análisis y gestión de riesgos en los SI, definiéndose los distintos términos empleados en las mismas. En el Capítulo 6 se describe la borrosificación de la metodología de análisis y gestión de riesgos MAGERIT, utilizada en España, mientras que el Capítulo 7 se centra en el problema de selección de salvaguardas para reducir el riesgo ante las amenazas mediante el uso de metaheurísticas y progamación dinámica.

En el Capítulo 8 se muestra un ejemplo ilustrativo de una unidad administrativa que utiliza un SI para el desarrollo de sus tareas internas y para la prestación de servicios de atención administrativa a los ciudadanos (administración electrónica). Finalmente, presentamos las conclusiones de este trabajo e indicamos líneas futuras de investigación en el Capítulo 9. 


\section{Capítulo 2 OBJETIVOS}

El objetivo fundamental de esta tesis consiste en proponer un modelo de análisis y gestión de riesgos en los sistemas de información (SI) bajo un enfoque borroso. Este objetivo general se puede subdividir en los siguientes:

1. Definir y describir aquellos elementos de lógica borrosa necesarios en el modelo, estableciendo un estado del arte sobre números borrosos trapezoidales, aritméticas borrosas y funciones de similitud de dichos números.

2. Proponer un método interactivo analista-experto mediante el cual podamos extraer el juicio probabilístico borroso del experto sobre un evento cualquiera, evitando sesgos. Para ello, hemos de describir los elementos básicos de la Teoría de la Probabilidad Lingüística, extendiendo a la Teoría Clásica de la Probabilidad.

3. Proponer una aritmética adecuada que mejore las aritméticas borrosas usuales, simplificando los algoritmos que vamos a utilizar en el análisis de riesgos en los SI.

4. Proponer una función de similitud que mejore las funciones señaladas en la literatura, aportando propiedades deseables y corrigiendo los inconvenientes encontrados en otras funciones de similitud. Probar la bondad de la función propuesta mediante un análisis comparativo.

5. Aplicar los elementos anteriores en el ámbito del análisis y la gestión de riesgos en los SI.

6. Describir técnicas de selección óptima de salvaguardas en la gestión del riesgo en los SI mediante metaheurísticas y programación dinámica. 


\section{Capítulo 3 ELEMENTOS DE LÓGICA BORROSA EN EL MODELADO DE LA INCERTIDUMBRE}

\subsection{Introducción}

El paradigma de la lógica borrosa comienza a partir de la Teoría de Conjuntos Borrosos, definiendo un conjunto $A$ de una categoría $\chi$ como un término lingüístico junto con una función $\mu_{A}: \chi \rightarrow[0,1]$ que indica el grado de pertenencia de cualquier elemento de $\chi$ al conjunto $A$. Esto nos permite modelar términos vagos o imprecisos. Por ejemplo, podemos definir el conjunto borroso $A=$ "Cercano al punto $(2,2)$ " de $\mathbb{R}^{2}$ (Figura 3.1.1) mediante la función de pertenencia (Figura 3.1.2)

$$
\mu_{A}(x, y)=e^{(x-2)^{2}+(y-2)^{2}} .
$$

Esto quiere decir que el punto $(2,2)$ tiene un grado de pertenencia $\mu_{A}(2,2)=1$ a "Cercano a $(2,2)$ ", mientras que el $(5,7)$ tiene un grado de pertenencia $\mu_{A}(5,7)=e^{-34}=$ $1.7 \times 10^{-15} \approx 0$. Sin embargo, la frontera entre "Cercano a $(2,2)$ " y "No cercano a $(2,2)$ " es borrosa.

La Teoría de Conjuntos Borrosos extiende a la Teoría Clásica de Conjuntos, que en lo sucesivo llamaremos Teoría de Conjuntos Nítidos, ya que un conjunto clásico es un caso degenerado de conjunto borroso con función de pertenencia

$$
\mu_{A}(x)=\left\{\begin{array}{ll}
1, & \text { si } x \in A \\
0, & \text { si } x \notin A
\end{array}\right. \text {. }
$$




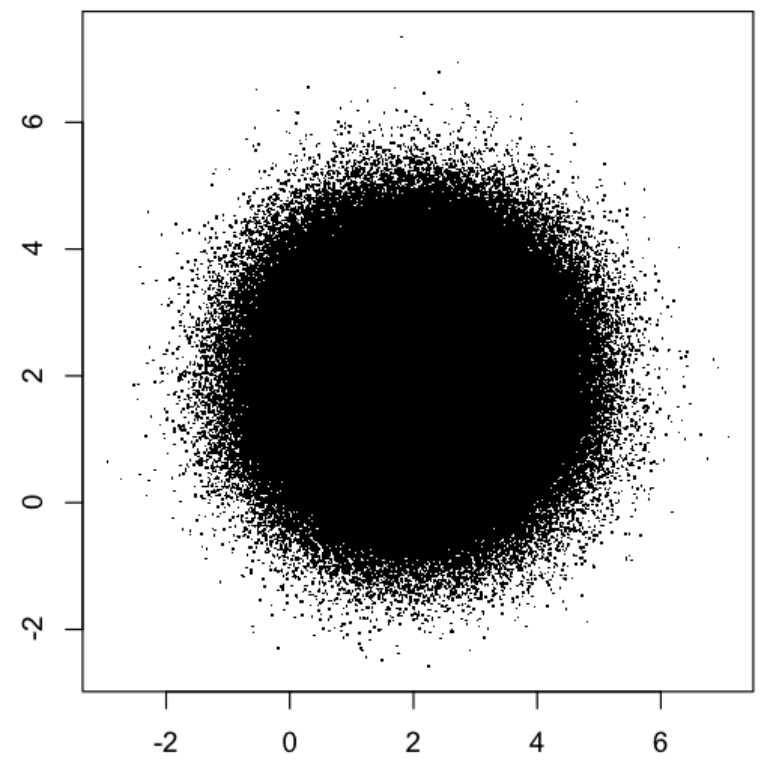

Figura 3.1.1: Conjunto borroso "Cercano a $(2,2)$ "

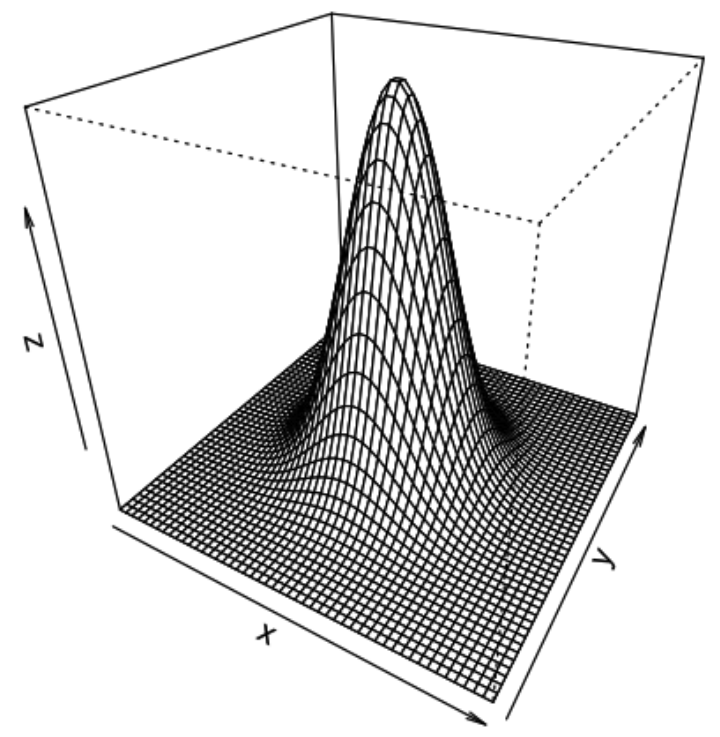

Figura 3.1.2: Función de pertenencia de "Cercano a $(2,2)$ " 
La Teoría de Conjuntos Borrosos, en muchos casos, es más natural y eficiente que la Teoría de Conjuntos Nítidos. Por ejemplo, pensemos en el conjunto de personas de estatura alta. La Teoría de Conjuntos Nítidos identifica una cota por debajo de la cual las personas ya no son consideradas altas. Supongamos que se considera que una persona es alta a partir de una estatura de $180 \mathrm{~cm}$. La Teoría de Conjuntos Nítidos considera "no alta" a una persona que mide $179 \mathrm{~cm}$, mientras que la Teoría de Conjuntos Borrosos considera que esta persona tiene un grado de pertenencia cercano a 1 al conjunto de las personas altas, y por tanto "tiene más de alta que de no alta”. Más formalmente, si asignamos una función de pertenencia

$$
\mu_{A}(x)=\left\{\begin{array}{cc}
0, & \text { si } x<170 \\
1-e^{-0.4(x-170)^{2}}, & \text { si } 170 \leq x
\end{array}\right.
$$

para el conjunto "personas altas", donde $x$ mide la estatura en $\mathrm{cm}$, entonces el grado de pertenencia de una persona con $179 \mathrm{~cm}$ de estatura es de 0.9983 , mientras que para una persona de $180 \mathrm{~cm}$ su grado de pertenencia es de 0.9999 .

Sin embargo, con la Teoría de Conjuntos Nítidos, el grado de pertenencia de una persona con $179 \mathrm{~cm}$ es de 0 (no es alta) y el de una persona con $180 \mathrm{~cm}$ es de 1 (es alta). Entonces, la Teoría de Conjuntos Borrosos representa mejor el razonamiento que la teoría clásica.

La Teoría de Conjuntos Borrosos fue introducida por primera vez en 1965 por Lotfy A. Zadeh [79], de la Universidad de California en Berkeley, aunque su aplicación natural, la Teoría de Controladores Borrosos, no fue presentada hasta 1973 [81]. Esta teoría, denominada recientemente por el propio Zadeh como Computación con Palabras [40], consiste en confeccionar una base de reglas en las que intervienen términos lingüísticos imprecisos para tomar decisiones automáticas que controlan un proceso determinado. Se han desarrollado aplicaciones inmediatas de la teoría en procesos de purificación del agua, sistemas de calefacción, aparcamiento automático y autoconducción inteligente de vehículos, análisis de riesgos, etcétera [40].

La Teoría de Conjuntos Borrosos junto con la Teoría de Controladores Borrosos forman la Lógica Borrosa. Esta disciplina tuvo dificultades para prosperar en sus orígenes, ya que 
únicamente la comunidad científica y técnica japonesa supo verla y acogerla como una disciplina que revolucionaría la ingeniería. Actualmente, empresas como NASA, Boeing, Ford o Bell utilizan la lógica borrosa en sus procesos, mientras que Zadeh recibe numerosos galardones honoríficos en todo el mundo por su invento.

De la Teoría de Conjuntos Borrosos se obtiene naturalmente una Teoría de Números Borrosos sin más que considerar $\chi=\mathbb{R}$. Esta teoría permite sintetizar en un único modelo las ventajas de sistemas cualitativos y cuantitativos, de modo que por un lado permite tratar la información en términos lingüísticos cualitativos y, por otro lado, permite el establecimiento de herramientas de cálculo (aritméticas) que permiten dar un tratamiento cuantitativo a la información.

Este enfoque es especialmente útil en los sistemas expertos en los que la información aportada es vaga o imprecisa. En esta dirección pueden verse, a modo de ejemplo, las redes bayesianas borrosas utilizadas por Halliwell [25].

En estos modelos, la información lingüística imprecisa aportada por los expertos es tratada por medio de una aritmética borrosa para obtener indicadores útiles que informen a los decisores sobre los procesos tratados. El valor final de estos indicadores no tiene por qué coincidir con una de las etiquetas lingüísticas de las que partíamos, y es aquí donde necesitaremos el uso de funciones de similitud de números borrosos para la asignación de una etiqueta.

En este capítulo describiremos las herramientas necesarias para el establecimiento de estos sistemas expertos borrosos y veremos una técnica de extracción (educción) del juicio probabilístico mediante un diálogo interactivo analista-experto.

En la Sección 3.2 exponemos las herramientas sobre números borrosos que vamos a utilizar a lo largo de esta tesis. En la Sección 3.3 exponemos el concepto de variable lingüistica. En la Sección 3.4 planteamos la necesidad de utilizar funciones de similitud de números borrosos trapezoidales generalizados y en la Sección 3.5 presentamos el concepto de variable aleatoria lingüistica y el método de educción de probabilidades. 


\subsection{Números borrosos. Aritmética y topología}

Como se ha indicado, un conjunto borroso $A$ de una categoría $\chi$ se define a partir de una función $\mu_{A}: \chi \rightarrow[0,1]$ que indica el grado de pertenencia de cada elemento de la categoría al conjunto $A$. En particular, si $\chi=\mathbb{R}$ podemos hablar de números borrosos:

Definición 3.1. Un número borroso normalizado $\tilde{A}$ se define por medio de una función de pertenencia $\mu_{\tilde{A}}: \mathbb{R} \longrightarrow[0,1]$ verificando las siguientes propiedades [82, 83, 84]:

1. $\max _{x \in \mathbb{R}}\left\{\mu_{\tilde{A}}(x)\right\}=1$.

2. $\forall x \leq y \leq z$ se tiene $\mu_{\tilde{A}}(y) \geq \min \left\{\mu_{\tilde{A}}(x), \mu_{\tilde{A}}(z)\right\}$.

3. El soporte de $\mu_{\tilde{A}}$ es acotado.

4. $\forall \alpha>0$ el conjunto $\left\{x \in \mathbb{R}: \mu_{\tilde{A}}(x) \geq \alpha\right\}$ es cerrado.

Además, si $\max _{x \in \mathbb{R}}\left\{\mu_{\tilde{A}}(x)\right\}=w \in[0,1)$ el número se denomina generalizado [9].

Existe una serie de elementos notables en los conjuntos y números borrosos que pasamos a definir.

Definición 3.2. Se define el soporte de $\tilde{A}$ como el conjunto de elementos cuyo grado de pertenencia a $\tilde{A}$ es no nulo: $\operatorname{sop}(\tilde{A})=\left\{x \in \chi: \mu_{\tilde{A}}(x)>0\right\}$.

Definición 3.3. Se define el núcleo de $\tilde{A}$ como el conjunto de elementos cuyo grado de pertenencia a $\tilde{A}$ es 1: Núcleo $(\tilde{A})=\left\{x \in \chi: \mu_{\tilde{A}}(x)=1\right\}$.

Definición 3.4. Se define la altura de $\tilde{A}$ como $w_{\tilde{A}}=\max _{x \in \chi}\left\{\mu_{\tilde{A}}(x)\right\}$.

Definición 3.5. Dado $\alpha \in[0,1]$, se define el $\alpha$-corte de $\tilde{A}$ como :

$$
\widetilde{A}_{\alpha}=\left\{\begin{array}{ll}
\left\{x \in \chi: \mu_{\widetilde{A}}(x) \geq \alpha\right\}, & \text { si } \alpha \in(0,1] \\
\overline{\left\{x \in \chi: \mu_{\widetilde{A}}(x)>0\right\}}, & \text { si } \alpha=0
\end{array},\right.
$$

donde $\bar{X}$ es la clausura o el cierre topológico del conjunto $X$.

En particular, los $\alpha$-cortes de cualquier número borroso son intervalos reales cerrados [2]. En la Figura 3.2.1 se señalan estos elementos sobre un número borroso. Esta figura 
corresponde a un número borroso con función de pertenencia

$$
\mu_{\widetilde{A}}(x)=\left\{\begin{array}{cl}
0, & \text { si } x<0.1 \\
\frac{x-0.1}{0.1}, & \text { si } 0.1 \leq x<0.2 \\
1, & \text { si } 0.2 \leq x<0.3 \\
\frac{0.4-x}{0.1}, & \text { si } 0.3 \leq x<0.4 \\
0, & \text { si } 0.4 \leq x
\end{array}\right.
$$

a la que llamamos función de pertenencia trapezoidal.

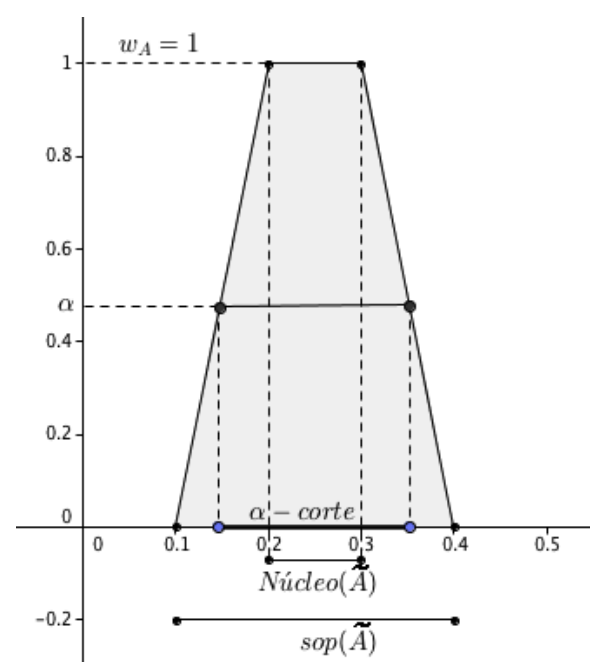

Figura 3.2.1: Elementos de un conjunto borroso.

Otros tipos de funciones de pertenencia sobre la recta real que se utilizan con frecuencia son:

1. La función de pertenencia triangular:

$$
\mu_{\widetilde{A}}(x)=\left\{\begin{array}{cl}
0, & \text { si } x<a \\
\frac{x-a}{b-a}, & \text { si } a \leq x \leq b \\
\frac{c-x}{c-b}, & \text { si } b \leq x \leq c \\
0, & \text { si } x \geq c
\end{array}\right.
$$

2. La función gaussiana: $\mu_{\widetilde{A}}(x)=e^{-k(x-m)^{2}}$.

3. La función sigmoidea: $\mu_{\widetilde{A}}(x)=\frac{1}{1+e^{-k(x-b)}}$. 
Estas funciones de pertenencia se muestran en la Figura 3.2.2

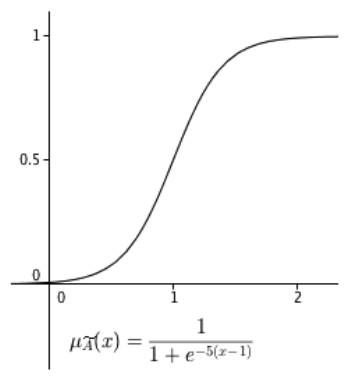

(a) Función sigmoidea.

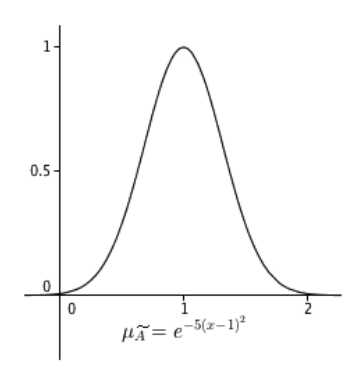

(b) Función gaussiana.

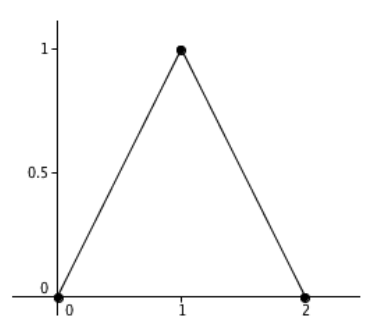

(c) Función triangular

Figura 3.2.2: Funciones de pertenencia habituales

Estamos particularmente interesados en los números borrosos trapezoidales normalizados, es decir, aquéllos cuya función de pertenencia es trapezoidal y de altura 1, ya que éstos se pueden representar mediante una tupla $\left(a_{1}, a_{2}, a_{3}, a_{4}\right)$ con $a_{1} \leq a_{2} \leq a_{3} \leq a_{4}$ junto con la función de pertenencia

$$
\mu_{\widetilde{A}}=\left\{\begin{array}{cl}
0, & \text { si } x<a_{1} \\
\frac{x-a_{1}}{a_{2}-a_{1}}, & \text { si } a_{1} \leq x<a_{2} \\
1, & \text { si } a_{2} \leq x<a_{3} \\
\frac{x-a_{4}}{a_{3}-a_{4}}, & \text { si } a_{3} \leq x \leq a_{4} \\
0, & \text { si } a_{4}<x
\end{array}\right.
$$

Denotaremos como $\mathbb{R}^{\mathcal{T F}}$ al conjunto formado por todos los números borrosos trapezoidales normalizados, y por $\mathbb{R}^{\mathcal{T F \mathcal { G }}}$ a los números borrosos trapezoidales generalizados, que representaremos mediante una tupla $\left(a_{1}, a_{2}, a_{3}, a_{4} ; w\right)$ considerando la altura $w \in[0,1]$ del número borroso.

Nótese que, si además $a_{2}=a_{3}$, entonces la función de pertenencia tiene forma triangular, por lo que los números borrosos trapezoidales extienden a los triangulares. 
Representar los números borrosos trapezoidales mediante tuplas $\left(a_{1}, a_{2}, a_{3}, a_{4}\right)$ nos permitirá definir operaciones aritméticas de una forma sencilla, así como extraer información borrosa de los expertos en los sistemas de ayuda a la decisión.

Los números borrosos normalizados extienden de modo natural a los números reales: El número $a \in \mathbb{R}$ se puede representar mediante la función de pertenencia

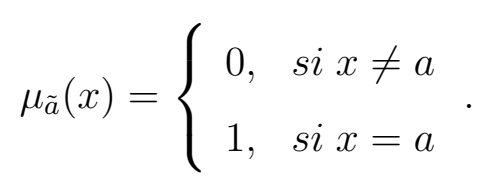

También los intervalos de números reales se pueden representar por medio de números borrosos: El intervalo $A=[a, b]$ se puede representar por la función de pertenencia

$$
\mu_{\tilde{A}}(x)=\left\{\begin{array}{ll}
0, & \text { si } x \notin[a, b] \\
1, & \text { si } x \in[a, b]
\end{array} .\right.
$$

En particular, un número real a se puede escribir como número borroso trapezoidal normalizado como $\tilde{a}=(a, a, a, a)$, y el intervalo $[a, b]$ de números reales se puede escribir como $\tilde{A}=(a, a, b, b)$.

\section{Aritmética, Orden y Relación de Subsumción}

Las operaciones aritméticas usuales sobre los números borrosos son las siguientes:

Dados dos números borrosos trapezoidales normalizados $\tilde{A}, \tilde{B} \in \mathbb{R}^{\mathcal{T F}}$ :

$$
\begin{aligned}
& \mu_{\tilde{A} \oplus \tilde{B}}(z)=\sup _{x+y=z} \min \left\{\mu_{\tilde{A}}(x), \mu_{\tilde{B}}(y)\right\} \\
& \mu_{\tilde{A} \otimes \tilde{B}}(z)=\sup _{x y=z} \min \left\{\mu_{\tilde{A}}(x), \mu_{\tilde{B}}(y)\right\} \\
& \mu_{\tilde{A} \ominus \tilde{B}}(z)=\sup _{x-y=z} \min \left\{\mu_{\tilde{A}}(x), \mu_{\tilde{B}}(y)\right\} \\
& \mu_{\tilde{A} \oslash \tilde{B}}(z)=\sup _{x / y=z} \min \left\{\mu_{\tilde{A}}(x), \mu_{\tilde{B}}(y)\right\}
\end{aligned}
$$

En particular, en los números borrosos trapezoidales normalizados estas operaciones se pueden escribir de la siguiente forma:

$$
\text { - }\left(a_{1}, b_{1}, c_{1}, d_{1}\right) \oplus\left(a_{2}, b_{2}, c_{2}, d_{2}\right)=\left(a_{1}+a_{2}, b_{1}+b_{2}, c_{1}+c_{2}, d_{1}+d_{2}\right)
$$


- $\left(a_{1}, b_{1}, c_{1}, d_{1}\right) \ominus\left(a_{2}, b_{2}, c_{2}, d_{2}\right)=\left(a_{1}-d_{2}, b_{1}-c_{2}, c_{1}-b_{2}, d_{1}-a_{2}\right)$

- $\left(a_{1}, b_{1}, c_{1}, d_{1}\right) \otimes\left(a_{2}, b_{2}, c_{2}, d_{2}\right)=\left(a_{1} \times a_{2}, b_{1} \times b_{2}, c_{1} \times c_{2}, d_{1} \times d_{2}\right)$

- $\left(a_{1}, b_{1}, c_{1}, d_{1}\right) \oslash\left(a_{2}, b_{2}, c_{2}, d_{2}\right)=\left(a_{1} / d_{2}, b_{1} / c_{2}, c_{1} / b_{2}, d_{1} / a_{2}\right)$ cuando tenga sentido.

Además, el conjunto de los números borrosos dispone de un orden parcial dado por:

$$
\tilde{A} \preceq \tilde{B} \Longleftrightarrow \tilde{A}=\tilde{\min }\{\tilde{A}, \tilde{B}\} \Longleftrightarrow \mu_{\tilde{A}}(z)=\sup _{\min \{x, y\}=z} \min \left\{\mu_{\tilde{A}}(x), \mu_{\tilde{B}}(y)\right\} \forall z \in \mathbb{R} .
$$

Tanto las operaciones aritméticas como el orden definido extienden a las operaciones aritméticas y al orden (total) [25] de los números reales.

Dados dos números borrosos $\tilde{A}$ y $\tilde{B}$, se dice que $\tilde{A}$ está subsumido en $\tilde{B}$, y se denota por $\tilde{A} \subseteq \tilde{B}$, si $\mu_{\tilde{A}}(x) \leq \mu_{\tilde{B}}(x) \forall x \in \mathbb{R}$.

\section{Topología de los números borrosos normalizados}

Dado un espacio métrico $(X, d)$, y dados $A, B \subseteq X$ cerrados y acotados, se define la distancia de Hausdorff entre $A$ y $B$ como:

$$
d_{H}(A, B)=\inf \left\{r>0:\left[A \subseteq \bigcup_{x \in B} B(x, r)\right] \bigwedge\left[B \subseteq \bigcup_{x \in A} B(x, r)\right]\right\}
$$

donde $B(x, r)=\{y \in X: d(x, y) \leq r\}$.

Si denotamos por $F_{X}$ al conjunto de todos los subconjuntos cerrados y acotados de $X$, entonces se verifica que si $(X, d)$ es un espacio métrico completo [25], entonces $\left(F_{X}, d_{H}\right)$ también es un espacio métrico completo, y puesto que todo subespacio métrico de un espacio métrico completo es también completo, se tiene que el conjunto de todos los $\alpha$ cortes definidos anteriormente forman, con la distancia de Hausdorff, un espacio métrico completo [25]. Esta es la base para dotar de una estructura de Banach a los números borrosos, como se muestra a continuación.

El conjunto de los números borrosos forma un espacio métrico completo con la siguiente distancia [19, 25]:

$$
d_{\infty}: \mathbb{R}^{F} \times \mathbb{R}^{F} \longrightarrow \mathbb{R}
$$




$$
d_{\infty}(\tilde{A}, \tilde{B})=\sup _{\alpha \in[0,1]} d_{H}\left(\tilde{A}_{\alpha}, \tilde{B}_{\alpha}\right)
$$

Esta estructura permite definir la convergencia de una sucesión de números borrosos o el concepto de serie convergente, fundamental para poder definir un espacio probabilístico borroso, dado que el tercer axioma de Kolmogorov ${ }^{1}$ trata con series convergentes.

\subsection{Modelado lingüístico borroso}

El uso de números borrosos y términos lingüísticos para la confección de sistemas expertos en los que no es posible aportar información nítida, o en los que es necesario mezclar la información nítida con información borrosa (recordemos que los números reales se inyectan en los borrosos), constituye lo que podemos denominar modelado lingüistico borroso [22]. En esta sección vamos a identificar cuáles son los elementos de un modelo lingüístico borroso y cuáles son algunos de los problemas que los analistas pueden encontrar al tratar de establecerlos.

Definición 3.5. Una variable lingüistica [40] es una tupla $(A, T(A), U, G, M)$ donde:

- $A$ es el nombre de la variable.

- $T(A)$ es el conjunto de términos lingüísticos que puede tomar $A$.

- $U$ es el universo de discurso en el que valora $A$.

- $G$ es una regla sintáctica para la generación de los términos de $T(A)$.

- $M$ es una regla semántica para asociar un significado a cada valor.

Vamos a explicar cada uno de los elementos de esta tupla mediante un ejemplo. La velocidad de un automóvil es una variable cuantitativa que puede tomar los valores de 0 a $120 \mathrm{~km} / \mathrm{h}$. Si consideramos a la velocidad como una variable lingüística, este rango de valores constituye el universo de discurso $(U)$ de la variable, $T$ (velocidad) es un conjunto de términos lingüísticos que puede tomar, como "parado", "lento" o "extremadamente

\footnotetext{
${ }^{1}$ Dada una falimilia de eventos disjuntos dos a dos $E_{i} \in \mathcal{H}$ (con $E_{i} \in \mathcal{H}$ una $\sigma$-algebra del espacio muestral de un experimento aleatorio) con $i \in \mathbb{N}$, se tiene que $p\left(\bigcup_{i \in \mathbb{N}} E_{i}\right)=\sum_{i \in \mathbb{N}} p\left(E_{i}\right)$.
} 
rápido". G la constituyen los nombres elegidos para $T$ (velocidad) y $M$ son los números borrosos cuyas etiquetas son los términos lingüísticos de T(velocidad).

Un modelo lingüístico para el establecimiento de un sistema de ayuda a la decisión consta de dos partes [22]:

1. Un modelo de representación. Conjunto de términos lingüísticos y números borrosos que los representan $(G$ y $M)$.

2. Un modelo computacional. Conjunto de operadores de agregación, comparación y aritméticos útiles para tratar la información aportada por los expertos.

El modelo de representación constituye el problema de la granularidad de la imprecisión $[5,22]$. Consiste en determinar un número adecuado de términos lingüísticos y números borrosos suficientemente alto como para representar la precisión que el experto puede declarar y suficientemente bajo como para que el experto no dude entre dos términos consecutivos. Lo habitual es utilizar escalas con un número impar de términos, entre 5 y 9. La cardinalidad de este conjunto es la granularidad del modelo.

Además, es importante determinar la forma y tamaño que han de tener los números borrosos que vamos a utilizar. A números más borrosos (con un mayor soporte) menor granularidad. Veamos un ejemplo.

En Vicente et al. [63] puede verse un árbol de fallos (Figura 3.3.1) utilizado en el análisis de riesgos por Dokas et al. [17]. Los expertos deben asignar probabilidades de fallos a los eventos BE1, BE2 y BE3. A partir de estas probabilidades, por medio de la aritmética borrosa indicada en la sección anterior y siguiendo las puertas lógicas del árbol, se debe calcular la probabilidad de que falle el top event (TE).

Dokas et al. proponen la primera escala de la Figura 3.3.2, que puede verse con detalle en la Tabla 3.3.1, y suponen que los expertos asignan los términos lingüísticos "Bajo", "Medio" y "Alto" respectivamente a las probabilidades de fallo de los eventos B1, B2 y B3. Con su escala resulta que la probabilidad de fallo del top event (TE) es $(0.35,0.52$, $0.52,0.68)$.

Sin embargo, con la escala de Vicente et al. (segunda escala de la Figura 3.3.2, Tabla $3.3 .1)$, el resultado es $(0.26,0.41,0.59,0.73)$. De este modo, se ve que la elección de los números borrosos que se van a utilizar puede llevar a resultados muy diferentes. Además, 
la escala utilizada para un experto puede no ser la escala más adecuada para otro experto, puesto que es posible (y habitual) que dos expertos tengan visiones más o menos claras (nítidas) de la probabilidad de materialización de un evento.

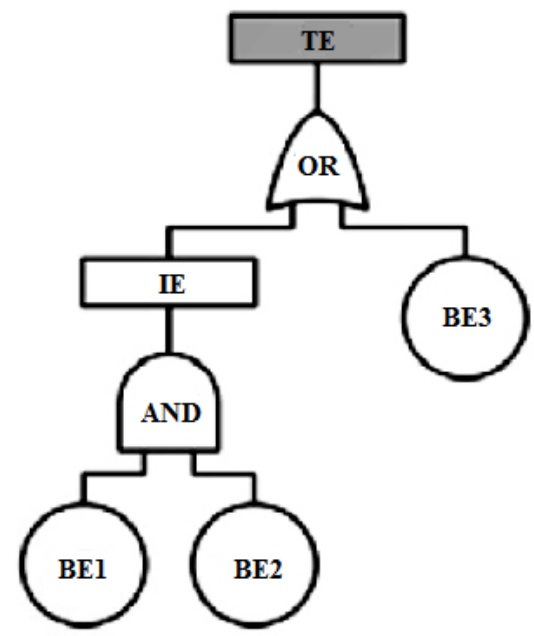

Figura 3.3.1: Árbol de fallos de Dokas et al. [17]

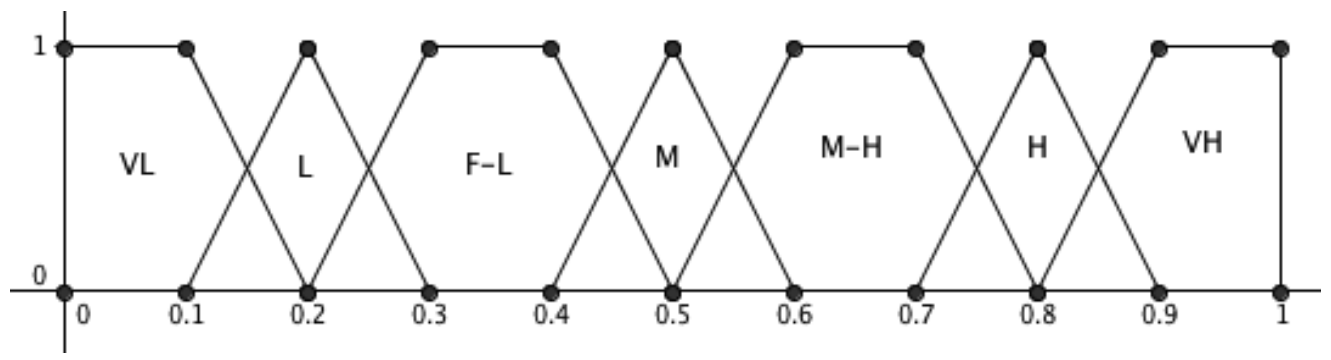

(a) Escala de Dokas et al. [17]

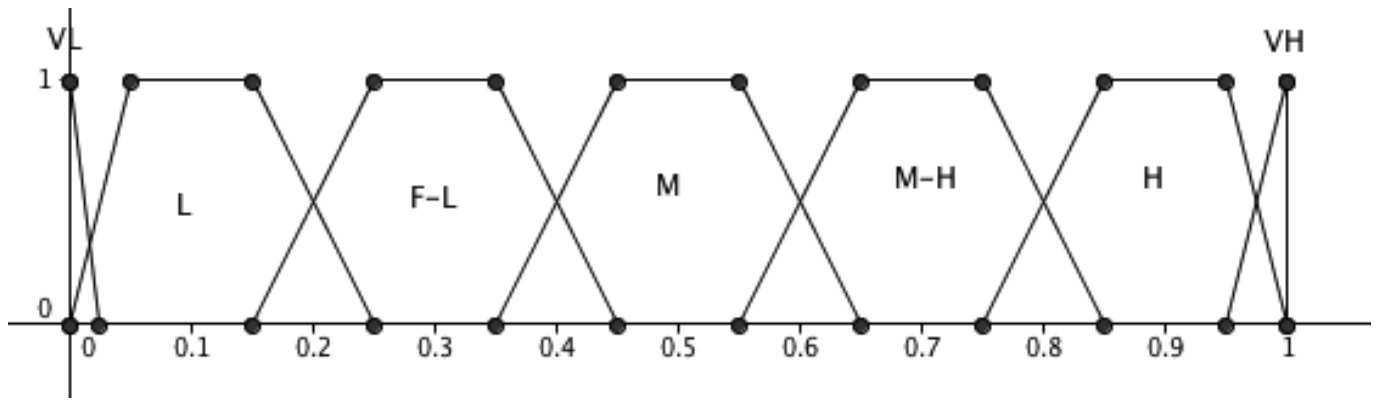

(b) Escala alternativa de Vicente et al. [61]

Figura 3.3.2: Posibles escalas para el árbol de fallos de Dokas et al.. 
Tabla 3.3.1: Escalas para la asignación de probabilidades.

\begin{tabular}{ccc}
\hline Término lingüístico $(\mathrm{G})$ & Dokas et al. [17] & Vicente et al. [63] \\
\hline \hline Muy Bajo (VL) & $(0,0,0.1,0.2)$ & $(0,0,0,0.025)$ \\
\hline Bajo (L) & $(0.1,0.2,0.2,0.3)$ & $(0,0.05,0.15,0.25)$ \\
\hline Medio Bajo (FL) & $(0.2,0.3,0.4,0.5)$ & $(0.15,0.25,0.35,0.45)$ \\
\hline Medio (M) & $(0.4,0.5,0.5,0.6)$ & $(0.35,0.45,0.55,0.65)$ \\
\hline Medio Alto (FH) & $(0.5,0.6,0.7,0.8)$ & $(0.55,0.65,0.75,0.85)$ \\
\hline Alto (H) & $(0.7,0.8,0.8,0.9)$ & $(0.75,0.85,0.95,1)$ \\
\hline Muy Alto (VH) & $(0.8,0.9,1,1)$ & $(0.95,1,1,1)$ \\
\hline
\end{tabular}

\subsection{El modelo computacional y la función de similitud}

En el ejemplo dado en la sección anterior ponemos de manifiesto no sólo el problema de la granularidad y la elección de números borrosos, sino el hecho de que los operadores dados por el modelo computacional sobre los números borrosos del modelo de representación no tienen por qué llevar a resultados en el mismo modelo de representación. De hecho, se observa que tanto si usamos la escala de Dokas et al. [17], como la propuesta en Vicente et al. [63], los números borrosos no están en sus respectivas escalas. En la Figura 3.4.1 observamos el resultado (rayado) sobre la escala de Vicente et al.

Entonces, hemos de trasladar el resultado al modelo de representación elegido. Esto se hace por medio de una función de similitud de números borrosos. Parece obvio que el resultado del árbol de fallos es una probabilidad media en la escala de Vicente et al. [63], pero esta obviedad se debe a la similitud que existe entre el número borroso correspondiente a la etiqueta "Medio" y el resultado obtenido. Precisamente, uno de los objetivos principales de esta tesis consiste en proponer una función que indique la similitud entre dos números borrosos trapezoidales, mejorando las funciones actuales. Profundizaremos en estas ideas en el Capítulo 4.

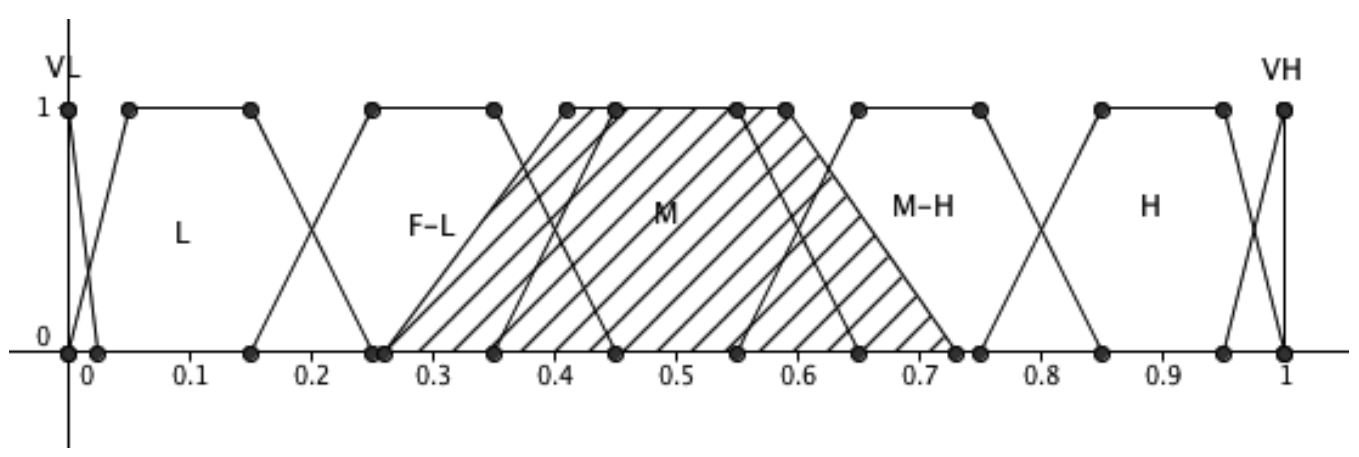

Figura 3.4.1: Resultado del árbol de fallos sobre la escala de Vicente et al. [61] 


\subsection{Educción de probabilidades mediante números bo- rrosos}

El uso de escalas de términos lingüísticos representados por números borrosos es muy común en los sistemas de ayuda a la decisión en ambiente borroso. Sin embargo, este tipo de escalas en el modelo de representación provocan una pérdida de información al discretizar los espacios de valoración y anclar la atención de los expertos y decisores sobre números borrosos previamente establecidos, con una amplitud de soporte y una forma que no han sido, en general, determinados por ellos, sino por el analista.

El uso de escalas de términos lingüísticos es útil al final de un proceso de educción y elicitación para normalizar los resultados de dichos procesos mediante funciones de similitud de números borrosos, y es fundamental en la implementación de sistemas de control borroso. Pero pedir a expertos y decisores que elijan entre cinco o siete números borrosos previamente establecidos para hacer sus valoraciones en los sistemas de ayuda a la decisión no es eficiente.

Por otro lado, la asignación directa de probabilidades no está en general recomendado por los protocolos usuales de educción de probabilidades subjetivas, como el SRI (Standford Research Institute)[43, 58, 59], ya que la asignación directa no previene los sesgos que se pudieran cometer.

En esta sección proponemos un nuevo método interactivo (indirecto) cuyo objetivo es extraer de decisores y expertos un número borroso que indique la probabilidad subjetiva de un suceso, y que permita el establecimiento de variables aleatorias borrosas sobre el espacio muestral de un experimento aleatorio.

Este método evita la discretización de los espacios de valoración y el uso de números borrosos con amplitud de soporte y forma previamente establecidos, permitiendo a cada decisor y experto una mayor o menor precisión en sus juicios probabilísticos y valoración de utilidades.

Además, estableceremos una función que mide la calidad del juicio probabilístico expresado por el experto mediante la agregación de dos componentes que miden la consistencia y la seguridad mostrada por el mismo durante el proceso de educción. 


\subsubsection{De la teoría clásica de la probabilidad a la teoría de la probabilidad lingüística}

Toda la Teoría Clásica de la Probabilidad se puede construir a partir de los bien conocidos axiomas de Kolmogorov.

Dados un espacio de eventos (espacio muestral) de un experimento aleatorio $\Omega$, y una $\sigma$-álgebra $\mathcal{H} \subseteq \mathbb{P}(\Omega)$ sobre $\Omega$, se dice que $p: \mathcal{H} \longrightarrow \mathbb{R}$ es una medida de probabilidad si verifica los siguientes axiomas:

- Axioma 1: $p(E) \in[0,1], \forall E \in \mathcal{H}$.

- Axioma 2: $p(\Omega)=1$.

- Axioma 3: Dada una familia de eventos disjuntos dos a dos $E_{i} \in \mathcal{H}$, con $i \in \mathbb{N}$, se tiene que $p\left(\bigcup_{i \in \mathbb{N}} E_{i}\right)=\sum_{i \in \mathbb{N}} p\left(E_{i}\right)$.

A partir de estos tres axiomas, se pueden demostrar las siguientes propiedades:

1. $p\left(E^{c}\right)=1-p(E), \forall E \in \mathcal{H}$, siendo $E^{c}$ el suceso compementario de $E$, es decir, tal que $E \bigcup E^{c}=\Omega$.

2. $p(\emptyset)=0$, siendo $\emptyset$ un suceso imposible.

3. $E \subseteq F \Longrightarrow p(E) \leq p(F)$.

4. $p(E \bigcup F)=p(E)+p(F)-p(E \bigcap F)$.

El tercer axioma de Kolmogorov implica que la serie $\sum_{i \in \mathbb{N}} p\left(A_{i}\right)$ debe ser convergente. Podemos utilizar términos de convergencia gracias a que en $\mathbb{R}$, y en particular en $[0,1]$, tenemos una estructura de espacio métrico dado por la distancia euclídea $d(x, y)=|x-y| \forall x, y \in$ $\mathbb{R}$. Además, el espacio $(\mathbb{R}, d)$ es un espacio métrico completo (toda sucesión de Cuachy en $(\mathbb{R}, d)$ es convergente).

Un espacio probabilístico $(\Omega, \mathcal{H}, p)$ es una terna formada por el espacio de eventos $\Omega$ de un experimento aleatorio, una $\sigma$-álgebra $\mathcal{H}$ sobre el espacio de eventos y una probabilidad $p$ sobre $\mathcal{H}$.

Una vez establecido un espacio probabilístico $(\Omega, \mathcal{H}, p)$ se pueden definir variables aleatorias que modelicen cuantitativamente la incertidumbre asociada a los experimentos, 
se puede definir la probabilidad de un evento condicionado a la ocurrencia de otro, o se puede contrastar la veracidad de una afirmación, etcétera.

Nuestro objetivo en esta sección es extender la teoría clásica al paradigma borroso, de modo que podamos definir una variable aleatoria lingüística [70].

Las primeras ideas sobre probabilidades borrosas son de Zadeh [80]. Sin embargo, Zadeh asigna un valor nítido para dar la probabilidad a partir de la función de pertenencia de un evento borroso (definido por una función de pertenencia), de modo que utiliza las reglas de la probabilidad clásica con números reales de [0,1] a través de los axiomas de Kolmogorov. Enfoques similares, en los que una variable aleatoria borrosa se define como una función sobre un espacio clásico de probabilidad que valora en los números borrosos, pueden verse en [35, 36, 21, 48].

Nosotros queremos realizar el proceso contrario, es decir, asignar un número borroso como probabilidad de un suceso que no tiene por qué estar definido por medio de una función de pertenencia. En este sentido, utilizaremos la Teoría de la Probabilidad Lingüística desarrollada por Halliwell en su tesis doctoral [25].

Los tres axiomas de Kolmogorov se pueden extender a los números borrosos normalizados de la siguiente forma:

Un espacio probabilístico borroso es una terna $\left(\Omega, \mathcal{H}, p^{\mathcal{F}}\right)$ formada por el espacio de eventos $\Omega$ de un experimento aleatorio, una $\sigma$-álgebra $\mathcal{H}$ sobre el espacio de eventos y una función $p^{\mathcal{F}}: \mathcal{H} \longrightarrow \mathbb{R}^{\mathcal{F}}$, verificando:

- Axioma 1: $\tilde{0} \leq p^{\mathcal{F}}(E) \leq \tilde{1} \forall E \in \mathcal{H}$.

- Axioma $2: p^{\mathcal{F}}(\Omega)=\tilde{1}$.

- Axioma 3: Dada una familia de eventos disjuntos dos a dos $E_{i} \in \mathcal{H}$, con $i \in \mathbb{N}$, se tiene que $p^{\mathcal{F}}\left(\bigcup_{i \in \mathbb{N}} E_{i}\right) \subseteq \sum_{i \in \mathbb{N}} p^{\mathcal{F}}\left(E_{i}\right)$

La aritmética usual de números borrosos normalizados dada anteriormente carece de elementos inversos respecto a las operaciones $\oplus \mathrm{y} \otimes$. Esto imposibilita la adaptación de la Propiedad 2 dada anteriormente. Por tanto, es necesario incluir un cuarto axioma en la Teoría de la Probabilidad Lingüística, para que ésta pueda extender a la teoría clásica:

- Axioma 4: $p^{\mathcal{F}}\left(A^{c}\right)=\tilde{1} \ominus p^{\mathcal{F}}(A)$. 
Con estos cuatro axiomas se verifican las siguientes propiedades:

1. $p^{\mathcal{F}}(\emptyset)=\tilde{0}$ siendo $\emptyset$ un suceso imposible. Basta aplicar los axiomas 4 y 2 .

2. $E \subseteq F \Longrightarrow p^{\mathcal{F}}(E) \leq p^{\mathcal{F}}(F)$. La demostración puede verse en [25].

3. $p^{\mathcal{F}}(E \bigcup F) \subseteq p^{\mathcal{F}}(E)+p^{\mathcal{F}}(F)-p^{\mathcal{F}}(E \bigcap F)$. Similar al caso real.

Relación con la Probabilidad Clásica. Dada una medida de probabilidad clásica $p: \mathcal{H} \longrightarrow[0,1]$ entonces $p^{\mathcal{F}}: \mathcal{H} \longrightarrow[0,1]^{\mathcal{F}}$ definida por $p^{\mathcal{F}}(E)=p(\tilde{E})$ es una medida de probabilidad borrosa, y recíprocamente, dada una medida de probabilidad borrosa $p^{\mathcal{F}}: \mathcal{H} \longrightarrow[0,1]^{\mathcal{F}}$ tal que $p^{\mathcal{F}}(E)=p(\tilde{E})$ para algún $p(E) \in \mathbb{R}$, entonces $p$ es una medida de probabilidad clásica.

Variable Aleatoria Borrosa y Teorema de Representación. Una variable aleatoria borrosa es una función $X:\left(\Omega, \mathcal{H}, p^{\mathcal{F}}\right) \longrightarrow D_{X}$ de un espacio de probabilidad borroso $\left(\Omega, \mathcal{H}, p^{\mathcal{F}}\right)$ en un universo de discurso $D_{X}$ tal que $X^{-1}(x) \in \mathcal{H}, \forall x \in D_{x}$. En particular, si la imagen de $X$ es un conjunto numerable, entonces se dice que la variable es discreta.

La función de masa de una variable aleatoria discreta es una función $f: X \longrightarrow[0,1]^{\mathcal{F}}$ definida por $f(x)=p^{\mathcal{F}}(w \in \Omega: X(w)=x)$.

En la Teoría Clásica de la Probabilidad es conocido que un conjunto de valores $\pi_{i} \in$ $[0,1]$ numerable, tal que $\sum_{i \in \mathbb{N}} \pi_{i}=1$, es la función de probabilidad de una variable aleatoria discreta $\chi$ que tiene un conjunto numerable de estados $d_{i}$ con probabilidad $P_{\chi}\left(d_{i}\right)=\pi_{i}$. El equivalente borroso de este teorema es el siguiente:

Teorema de representación [25] Si $D=\left\{d_{i}: i \in I\right\}$ es un conjunto finito no vacío y $\left\{\tilde{\pi}_{i}: i \in I\right\}$ es un conjunto de números borrosos tales que $\forall i \in I \tilde{0} \leq \tilde{\pi}_{i} \leq \tilde{1}$ y $\tilde{\pi}_{i} \subseteq$ $\tilde{1} \ominus\left[\underset{j \neq i}{\oplus} \tilde{\pi}_{j}\right] \in \mathbb{R}^{F}$, entonces existe un espacio probabilístico borroso $\left(\Omega, \mathcal{H}, p^{\mathcal{F}}\right)$ y una variable aleatoria borrosa discreta $X$ sobre este espacio probabilístico tal que su función de masa es

$$
f(d)=\left\{\begin{array}{lr}
\tilde{\pi}_{i}, & \text { si } d=d_{i} \text { para algún } i \in I \\
\tilde{0}, & \text { en otro caso }
\end{array}\right.
$$

Ejemplos. A continuación, describimos la función de masa borrosa de algunas variables sencillas. 
1. Los números borrosos triangulares $\widetilde{\pi}_{1}=(0,0.1,0.2), \tilde{\pi}_{2}=(0.25,0.3,0.35)$ y $\widetilde{\pi}_{3}=$ $(0.55,0.6,0.65)$ forman la función de masa de una variable aleatoria discreta que tiene tres estados con probabilidades $\widetilde{\pi}_{1}, \widetilde{\pi}_{2}$ y $\widetilde{\pi}_{3}$, respectivamente. Probabilidades similares se pueden expresar mediante los números trapezoidales $\widetilde{\pi}_{1}=(0,0.05,0.15,0.2)$, $\widetilde{\pi}_{2}=(0.25,0.27,0.33,0.35)$ у $\widetilde{\pi}_{3}=(0.55,0.57,0.63,0.65)$.

2. Los números borrosos trapezoidales $\widetilde{\pi}_{1}=\widetilde{\pi}_{2}=(0.35,0.45,0.55,0.65)$ forman la función masa de una variable aleatoria binaria en la que los sucesos "éxito" y "fracaso" tienen la misma probabilidad de ocurrencia. Del mismo modo, $\widetilde{\pi}_{1}=\widetilde{\pi}_{2}=$ $(0.5,0.5,0.5,0.5)$ es la función de masa de una variable dicotómica en la que los sucesos elementales (complementarios) tienen la misma probabilidad de ocurrencia. Este segundo caso constituye una percepción nítida de la probabilidad, de modo que el primer caso es más borroso y, por tanto, la expresión de la probabilidad mediante estos valores es de menor calidad que la expresión de la probabilidad mediante el segundo caso. Nos ocuparemos de esta cuestión más adelante.

3. Nótese que si los dos sucesos de un experimento aleatorio dicotómico tienen la misma probabilidad de ocurrencia, entonces la probabilidad de ambos debe verse con la misma borrosidad, ya que de la probabilidad de uno se deduce la del contrario. Es decir, en este caso, no puede ocurrir, por ejemplo, que $\widetilde{\pi}_{1}=(0.35,0.45,0.55,0.65)$ y $\widetilde{\pi}_{2}=(0.3,0.45,0.55,0.7)$.

4. Los números borrosos trapezoidales $\widetilde{\pi}_{1}=\widetilde{\pi}_{2}=\widetilde{\pi}_{3}=\left(\frac{1}{4}, \frac{6}{21}, \frac{8}{21}, \frac{1}{2}\right)$ forman la función de masa de una variable aleatoria discreta que tiene tres estados con la misma probabilidad.

\subsubsection{Asignación de probabilidades mediante apuestas y loterías}

El protocolo Stanford Research Institute (SRI) [43], aunque relativamente antiguo, es el protocolo más influyente en la elicitación individual de los juicios probabilísticos de expertos. Algunas guías recientes que toman como base este protocolo se pueden consultar en $[58,59]$.

El protocolo original consta de cinco fases, aunque ha sido ampliado a siete fases a principios de la década de 1990 para incluir asignación de juicio experto de manera 
colectiva [42].

De forma resumida, las cinco fases en que se divide el protocolo individual SRI son las siguientes:

1. Fase de Motivación. El analista introduce al experto en la tarea de asignación de probabilidades, tratando de hacerle comprender la importancia de la misma. Se explican superficialmente algunos métodos que se pueden utilizar o los tipos de sesgos que se pueden cometer.

2. Fase de Estructuración. Se trata de clarificar y definir las variables que se van a tratar, las escalas que se van a utilizar, si es necesario descomponer el proceso teniendo en cuenta dependencias entre algunas variables...

3. Fase de Condicionamiento. Se trata de explorar en profundidad los sesgos que se pudieran cometer para evitarlos en la fase de codificación de probabilidades.

4. Fase de Codificación. En esta fase se pone en práctica un método conveniente de elicitación de probabilidades.

5. Fase de Verificación. Se trata de contrastar si el experto está realmente de acuerdo con los juicios probabilísticos extraídos en la fase anterior.

Otros protocolos derivados del SRI son el SNL/NUREG-1150 [57] de la U.S. Nuclear Regulatory Commission y el KEEJAM [15] del Joint Research Center de la Comisión Europea.

Como vemos, la elicitación de probabilidades se realiza en la fase de codificación del protocolo SRI. Mediante la asignación de probabilidades subjetivas, se trata de establecer la función de probabilidad en el caso de una variable discreta y la función de distribución en el caso de que la variable aleatoria sea continua. En ambos casos se pueden desarrollar métodos directos o indirectos.

Los métodos directos para la derterminación de una función de probabilidad, en el caso discreto, consisten en encontrar un sistema completo de sucesos disjuntos dos a dos, y preguntar directamente al experto por la probabilidad de cada uno de los sucesos que forman dicho sistema. Es necesario garantizar los axiomas de Kolmogorov, ya que, de lo contrario, el experto no es coherente en sus juicios probabilísticos. 
En el caso continuo, los métodos directos consisten en preguntar al decisor por algunos percentiles de la distribución de probabilidad que se está elicitando.

Estos métodos directos son muy rápidos y fáciles de implementar. Sin embargo, tienen el inconveniente de que no evitan los sesgos que se pueden producir durante el proceso de educción.

Los métodos indirectos, en el caso discreto, consisten en presentarle al experto la posibilidad de participar en dos juegos diferentes en los cuales se ganan o se pierden determinadas cantidades monetarias con ciertas probabilidades, comparadas con las probabilidades del suceso bajo estudio. El experto debe elegir en qué juego desea participar, y si es coherente elegirá siempre aquella alternativa que le resulte más favorable. El analista cambiará iterativamente las condiciones de los juegos propuestos y aprovechará las respuestas del decisor en cada iteración para inferir la probabilidad de $A$. Se desarrolla así un proceso que culmina con un par de juegos entre los que el experto es indiferente, y en este momento resulta fácil dar un valor para la probabilidad de $A$.

El analista puede cambiar las condiciones de los juegos modificando cantidades monetarias o probabilidades. Si cambia cantidades monetarias, el método se denomina de educción mediante apuestas. Si se modifican las probabilidades, se denomina de educción mediante loterías.

\section{Asignación basada en apuestas}

Para asignar las probabilidades de un suceso $A$ mediante este método (Figura 3.5.1) tomamos dos cantidades monetarias $x$ e $y$ y presentamos al experto las siguientes apuestas:

- Primera Apuesta:

- Si ocurre el suceso $A$ usted gana $x$.

- Si no ocurre $A$, usted pierde $y$ (gana $-y$ ).

- Segunda Apuesta:

- Si ocurre $A$, usted pierde $x$ (gana $-x)$.

- Si no ocurre $A$, usted gana $y$. 


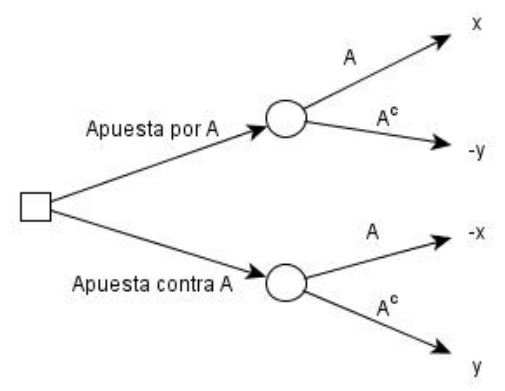

Figura 3.5.1: Juego de apuestas

Ahora se le pregunta qué apuesta prefiere. Si ambas apuestas son indiferentes para el decisor entonces la ganancia esperada en ambas es la misma:

$$
x P(A)-y P\left(A^{c}\right)=-x P(A)+y P\left(A^{c}\right),
$$

de donde

$$
P(A)=\frac{y}{x+y} .
$$

Si prefiere la primera apuesta entonces la ganancia esperada de dicha apuesta es mayor que la ganancia esperada de la segunda y, por tanto:

$$
x P(A)-y P\left(A^{c}\right)>-x P(A)+y P\left(A^{c}\right),
$$

de donde

$$
P(A)>\frac{y}{x+y} \text {. }
$$

En este caso, habría que aumentar el valor de $y$ o reducir el valor de $x$ y volver a presentar las apuestas al decisor. El proceso se repite hasta encontrar dos apuestas indiferentes que nos permitan asignar la probabilidad de $A$.

\section{Asignación basada en loterías.}

Si en lugar de cambiar los valores monetarios modificamos las probabilidades obtenemos el método basado en loterías (Figura 3.5.2), que consiste en lo siguiente:

Se consideran un premio muy bueno, T, y un premio muy malo, W, y a continuación, se presentan al experto las siguientes loterías:

- Primera Lotería:

- Si ocurre el suceso $A$, usted gana el premio T. 
- Si no ocurre el suceso $A$, usted gana el premio W.

- Segunda Lotería:

- Con probabilidad $p$ usted gana el premio T.

- Con probabilidad $1-p$ usted gana el premio W.

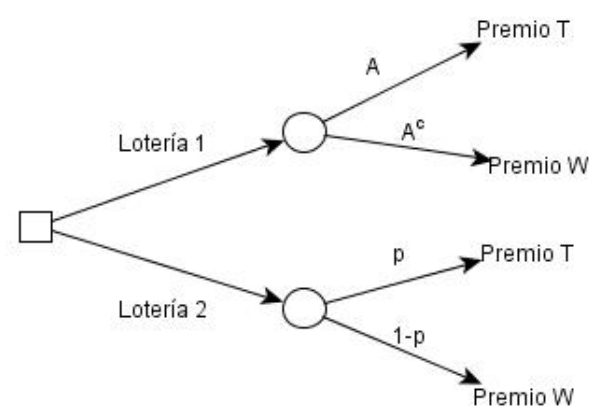

Figura 3.5.2: Juego de loterías

A la segunda lotería se la suele denominar lotería de referencia.

Si el experto prefiere la primera lotería es porque piensa que $P(A)>p$, por tanto, debemos incrementar $p$. Si prefiere la segunda lotería es porque piensa que $P(A)<p$ y debemos reducir $p$. Así hasta que encuentre dos loterías que sean indiferentes para el experto. Llegado ese momento $P(A)=p$.

Para este segundo método es muy común utilizar artilugios físicos que permitan al experto visualizar la magnitud de las probabilidades que se le están ofreciendo. El artilugio más usual es el de la ruleta de la probabilidad (Figura 3.5.3), que consiste en un círculo con dos sectores circulares que designan $p$ y $1-p$. Según las respuestas del experto, el radio que delimita los sectores $p$ y $1-p$ se gira para ampliar o reducir las probabilidades ofrecidas en el proceso iterativo.

Una vez que hemos asignado la probabilidad a un suceso se puede continuar con otro suceso disjunto al anterior y así sucesivamente. Al final, todas las probabilidades deben sumar 1. Si no fuera así el experto no sería coherente ya que estamos definiendo la función de masa subjetiva de una variable aleatoria discreta.

Los métodos indirectos de apuestas y loterías también son útiles en la elicitación de las funciones de distribución en el caso continuo. Estos métodos consisten en determinar 
las probabilidades acumuladas mediante el uso de loterías y apuestas. Destacan el método fixed probability y el fixed variable value [1, 54].

Vemos que los métodos de loterías y apuestas son bastante usuales, tanto en la elicitación de probabilidades de variables discretas, como en variables continuas. De hecho, la mayoría de los programas de software de análisis de decisiones disponen de una ruleta de probabilidad que permite desarrollar estos métodos con el experto. Buenos ejemplos son GeNie, DATA, DPL y MSBN [74].

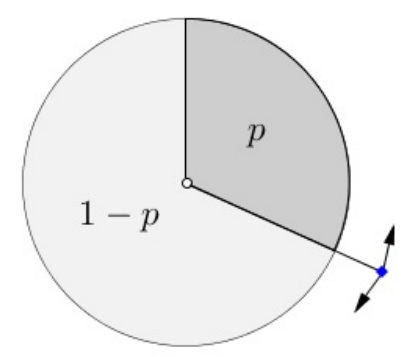

Figura 3.5.3: Ruleta de la probabilidad para asignar probabilidades

\subsubsection{Asignación de probabilidades borrosas mediante apuestas y loterías}

Los métodos de apuestas y loterías son elegantes, ingeniosos, útiles y fáciles de entender por parte de los expertos que deben asignar probabilidades, sin embargo, no están libres de inconvenientes. Veamos un ejemplo de entrevista analista-experto en el que se desarrolla el método de las loterías para asignar la probabilidad de un suceso $A$ :

- Pregunta 1 (analista): (primera rueda de la Figura 3.5.4) Si ocurre el suceso $A$ usted gana 1000 euros, y si no ocurre el suceso $A$ usted pierde 100 euros. Con probabilidad $p=0.25$ usted gana el premio 1000 euros, y con $1-p=0.75$ usted pierde 100 euros. ¿Qué lotería prefiere?

- Respuesta 1 (experto): La lotería 1.

El analista entiende que $p(A)>0.25$ e incrementa el valor de $p$. El diálogo continúa:

- Pregunta 2 (analista): (segunda rueda de la Figura 3.5.4) Si ocurre el suceso $A$ usted gana 1000 euros, y si no ocurre el suceso $A$ usted pierde 100 euros. Con probabilidad $p=0.5$ usted gana el premio 1000 euros, y con $1-p=0.5$ usted pierde 100 euros. ¿Qué lotería prefiere? 
- Respuesta 2 (experto): La lotería 1.

El analista entiende que $p(A)>0.5$ e incrementa el valor de $p$. El diálogo continúa:

- Pregunta 3 (analista): (tercera rueda de la Figura 3.5.4) Si ocurre el suceso $A$ usted gana 1000 euros, y si no ocurre el suceso $A$ usted pierde 100 euros. Con probabilidad $p=0.75$ usted gana el premio 1000 euros, y con $1-p=0.25$ usted pierde 100 euros. ¿Qué lotería prefiere?

- Respuesta 3 (experto): Me es indiferente.

Llegado este punto el analista, siguiendo el método de Savage, diría que la pobabilidad de $A$ es $P(A)=0.75$. Sin embargo ¿podemos inferir que $p(A) \neq 0.6,0.65,0.8,0.85$ ? Obviamente no porque no hemos preguntado por estos valores. Podría ocurrir que el experto también fuera indiferente a loterías de referencia con estos valores. Entonces, sería necesario hacer preguntas con valores en torno a 0.75 para ver hasta dónde llega la indiferencia del experto en la asignación de probabilidades.

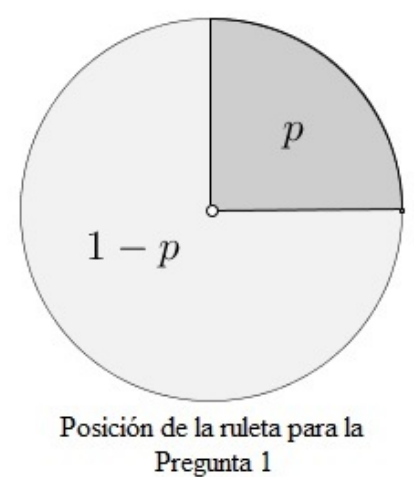

(A)

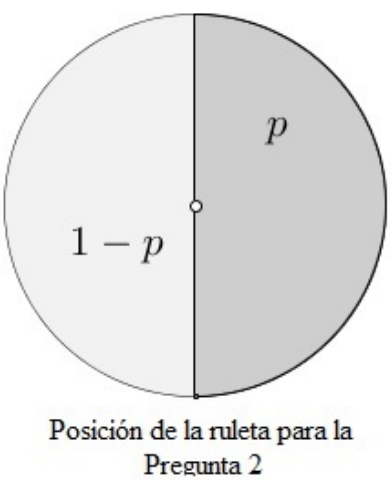

(B)

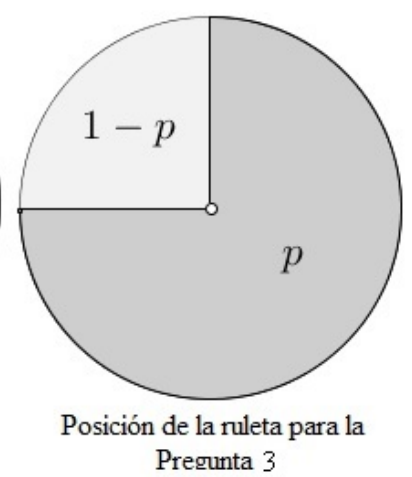

(C)

Figura 3.5.4: Posiciones de la ruleta de la probabilidad.

Así, si el experto no está completamente seguro (que es lo más normal) de que la probabilidad sea exactamente 0.75 , continuaríamos la entrevista con valores cercanos a 0.75 hasta romper la indiferencia tanto a la izquierda como a la derecha del 0.75 . Como consecuencia, obtendremos un sector circular en la ruleta de la fortuna (Figura 3.5.5) que constituye una frontera borrosa entre las probabilidades $P(A)$ y $P\left(A^{c}\right)$, indicando la vaguedad y la imprecisión del experto al asignar la probabilidad. 
El proceso terminará en un intervalo $[a, c]$ en el que debe estar la frontera entre ambos conjuntos.

Los protocolos usuales sobre educción de probabilidades, como el citado SRI, recomiendan utilizar varios métodos de asignación de probabilidades para evaluar la consistencia de las probabilidades dadas por el experto.

El método basado en apuestas expuesto anteriormente puede complementar a este método de las loterías para conseguir tal efecto. Si en cada pregunta cambiamos las cantidades (premios $\mathrm{T}$ y W) en lugar de las probabilidades, podemos obtener igualmente un intervalo de indiferencia en el que el experto es incapaz de identificar la probabilidad del suceso con seguridad.

Ambos métodos no tienen por qué dar exactamente los mismos resultados por motivos psicológicos. Sin embargo, si los intervalos obtenidos por ambos métodos son disjuntos, entonces las asignaciones aportadas por el decisor mediante ambos métodos no son consistentes, mientras que si existe intersección entre los intervalos que resultan de ambos métodos, entonces parece claro que el experto ve con más claridad que la probabilidad solicitada podría estar en esa intersección, y que fuera de ella, cabe la posibilidad de que esté pero con menor seguridad según nos alejamos de la intersección. Esto nos lleva al establecimiento de un número borroso trapezoidal.

Supongamos que del método de la lotería resulta $[a, c]$ y del método de la apuesta resulta $[b, d]$.

- Si $[a, c] \bigcap[b, d]=\emptyset$, entonces el experto es inconsistente.

- Si $[a, c] \bigcap[b, d] \neq \emptyset$, entonces:

- Si ninguno de los intervalos está contenido en el otro, podemos suponer sin pérdida de generalidad que $a<b \leq c<d$. Entonces, el experto piensa que la probabilidad del suceso es el número borroso trapezoidal $\tilde{\pi}=(a, b, c, d)$.

- Si uno de los intervalos está contenido en otro, podemos suponer sin pérdida de generalidad que $[a, c] \subseteq[b, d]$. Entonces, el experto piensa que la probabilidad del suceso es el número borroso trapezoidal $\tilde{\pi}=(b, a, c, d)$. 
Una vez que el experto ha asignado la probabilidad a un suceso, se le pide la probabilidad de otro suceso disjunto con el anterior, para lo cual se vuelven a desarrollar los dos métodos anteriores. Ahora bien, al igual que en el caso real exigíamos que la suma de las probabilidades de los sucesos (disjuntos dos a dos) fuera 1, en el paradigma borroso hemos de exigir que se verifiquen las condiciones del teorema de representación dado en la Sección 3.5.1, es decir, que

$$
\tilde{\pi}_{i} \subseteq \tilde{1} \ominus\left[\underset{j \neq i}{\oplus} \tilde{\pi}_{j}\right]
$$

siendo $\tilde{\pi}_{i}$ las probabilidades asignadas sucesivamente a los eventos (recordemos que la aritmética usual de números borrosos impide la resolución de ecuaciones sencillas ya que no existe elemento inverso respecto de las operaciones $\oplus \mathrm{y} \otimes)$.

De esta manera, en virtud del citado teorema, definimos una variable aleatoria discreta borrosa.

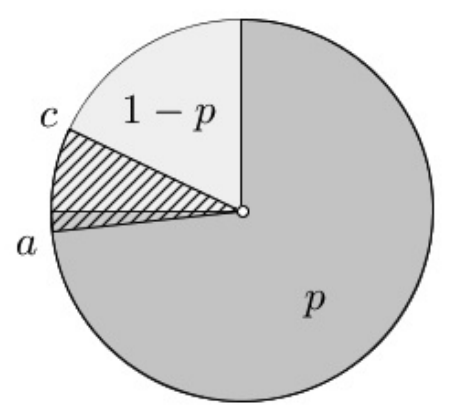

Figura 3.5.5: Frontera borrosa en la ruleta de la probabilidad.

\subsubsection{Calidad de la información. Medidas de borrosidad y de consistencia de los expertos}

Uno de los mayores inconvenientes de los sistemas expertos radica en los sesgos informativos que pueden cometer los expertos. Un trabajo pionero y tremendamente influyente en el estudio de estos sesgos corresponde a Tverky y Kahneman [56] que defienden que el ser humano construye heurísticas o reglas empíricas para asignar las probabilidades planteadas.

Estas reglas que, en general, pueden conducir a buenas estimaciones optimizando el esfuerzo dedicado. Sin embargo, pueden estar influidas por diversas circunstancias en el 


\subsection{EDUCCIÓN DE PROBABILIDADES MEDIANTE NÚMEROS BORROSOS}

momento en que se desarrollan las entrevistas analista-decisor, introduciendo sesgos que dificultan la bondad de la asignación.

Las heurísticas más comunes son las de disponibilidad (el experto sobrevalora la probabilidad de eventos que se han producido recientemente), representatividad (los expertos tienden a olvidar la representatividad de diversas clases de individuos en una población, sucesos raros con consecuencias importantes suelen ser más recordados, y su probabilidad se sobrestima) y de anclaje y ajuste (subestimación de sucesos disjuntos, sobreestimación de sucesos simultáneos, exceso de confianza,...).

La razón de utilizar los métodos indirectos de elicitación de probabilidades consiste, precisamente, en reducir la influencia de las heurísticas utilizadas por el experto, que le llevan a incluir sesgos en sus juicios probabilísticos. Además, los protocolos de elicitación usuales recomiendan el uso de varios métodos con objeto de poder contrastar la información suministrada con cada uno de ellos y poder discutir los resultados con el experto, o valorar la calidad de la información suministrada. De este modo, el método propuesto en la Sección 3.5.3 para la obtención de un número borroso que indique el juicio probabilístico del experto cumple con dichas recomendaciones. Además, la construcción de dicho número borroso permite valorar cuantitativamente la calidad de la información aportada.

A continuación, vamos a proponer dos medidas que informan sobre la consistencia y la seguridad del experto en su juicio probabilístico. Finalmente, agregaremos ambas medidas en una función que indique la calidad de la información aportada.

Consistencia del Experto. Como se indicaba en la Sección 3.5.3, el experto es inconsistente si $[a, c] \bigcap[b, d]=\emptyset$, pero si $[a, c] \bigcap[b, d]=\{\xi\}$ entonces el número borroso que designa el juicio probabilístico dado por el experto es un número borroso triangular $(a, \xi, d)$, ya que $\xi=b=c$.

Sin embargo ¿podemos decir que el experto es inconsistente si $[a, c] \bigcap[b, d]=\emptyset$, y no lo es si $[a, c] \bigcap[b, d]=\{\xi\}$ ? Obviamente no, ya que ambas situaciones son prácticamente iguales, de modo que los números borrosos triangulares también representan situaciones de inconsistencia, ya que la intersección de los intervalos es un único número real. De hecho, podemos afirmar que el número triangular representa el límite entre la consistencia y la inconsistencia en los juicios probabilísticos dados por los métodos de apuestas y loterías.

Por contra, si $[a, c]=[b, d]$ el experto ha sido completamente consistente, y el número 
borroso que designa su juicio probabilístico es un número rectangular (un intervalo nítido).

Entonces, la forma del número borroso (rectangular, trapezoidal propiamente dicho, o triangular) indica la consistencia del juicio proporcionado por el experto, y de este modo, podemos medir la consistencia mediante la siguiente función:

Dado un número borroso trapezoidal normalizado $\tilde{A}=(a, b, c, d) \in[0,1]^{\mathcal{T F}}$ que denota el juicio probabilístico de un experto para un evento determinado, llamaremos consistencia de $\tilde{A}$ a

$$
C(\tilde{A})=\left\{\begin{array}{cc}
\frac{c-b}{d-a}, & \text { si } \max \{(d-a),(c-b)\} \neq 0 \\
1, & \text { en otro caso }
\end{array} .\right.
$$

\section{Propiedades de la consistencia expresada por el experto:}

1. $C(\tilde{A}) \in[0,1] \forall \tilde{A} \in[0,1]^{\mathcal{T F}}$.

2. $C(\tilde{A})=1$ si y sólo si $\tilde{A}$ es un número nítido. En particular, la consistencia de cualquier intervalo real (visto como número borroso) contenido en $[0,1]$ es 1 .

3. $C(\tilde{A})=0$ si y sólo si $\tilde{A}$ es un número triangular.

4. $C$ es una función continua en $[0,1]^{\mathcal{T F}}$.

Demostración. Las tres primeras propiedades son triviales, ya que $0 \leq a \leq b \leq c \leq$ $d \leq 1$. Veamos la cuarta:

Sea $\left\{\tilde{X}_{n}\right\}_{n \in \mathbb{N}} \subset[0,1]^{\mathcal{T F}}$ una sucesión convergente:

$$
\tilde{X}_{n} \underset{n \rightarrow \infty}{\longrightarrow} \tilde{Y} \in[0,1]^{\mathcal{T F}} \Longleftrightarrow d_{\infty}\left(\tilde{X}_{n}, \tilde{Y}\right) \underset{n \rightarrow \infty}{\longrightarrow} 0
$$

Por tanto, $\sup _{\alpha \in[0,1]}\left\{d_{H}\left(\tilde{X}_{n \alpha}, \tilde{Y}_{\alpha}\right)\right\} \underset{n \rightarrow \infty}{\longrightarrow} 0 \mathrm{y}$, en particular, para $\alpha=1$ se tiene $d_{H}\left(\tilde{X}_{n 1}, \tilde{Y}_{1}\right) \underset{n \rightarrow \infty}{\longrightarrow} 0$, y para $\alpha=0, d_{H}\left(\tilde{X}_{n 0}, \tilde{Y}_{0}\right) \underset{n \rightarrow \infty}{\longrightarrow} 0$.

Luego,

$$
\begin{gathered}
d_{H}\left(\tilde{X}_{n 1}, \tilde{Y}_{1}\right)=\inf \left\{r>0:\left[\tilde{X}_{n 1} \subseteq \bigcup_{x \in \tilde{Y}_{1}} B(x, r)\right] \bigwedge\left[\tilde{Y}_{1} \subseteq \bigcup_{x \in \tilde{X}_{n 1}} B(x, r)\right]\right\} \underset{n \rightarrow \infty}{\longrightarrow} 0 \\
\Longleftrightarrow \tilde{X}_{n 1} \underset{n \rightarrow \infty}{\longrightarrow} \tilde{Y}_{1},
\end{gathered}
$$




$$
\begin{gathered}
d_{H}\left(\tilde{X}_{n 0}, \tilde{Y}_{0}\right)=\inf \left\{r>0:\left[\tilde{X}_{n 0} \subseteq \bigcup_{x \in \tilde{Y}_{0}} B(x, r)\right] \bigwedge\left[\tilde{Y}_{0} \subseteq \bigcup_{x \in \tilde{X}_{n 0}} B(x, r)\right]\right\} \underset{n \rightarrow \infty}{\longrightarrow} 0 \\
\Longleftrightarrow \tilde{X}_{n 0} \underset{n \rightarrow \infty}{\longrightarrow} \tilde{Y}_{0} .
\end{gathered}
$$

Para concluir, basta tener en cuenta que, por definición, $C\left(\tilde{X}_{n}\right)=\frac{l\left(\tilde{X}_{n 1}\right)}{l\left(\tilde{X}_{n 0}\right)}$ y $C(\tilde{Y})=$ $\frac{l\left(\tilde{Y}_{1}\right)}{l\left(\tilde{Y}_{0}\right)}$, donde $l$ denota la longitud del intervalo. Por tanto, $C\left(\tilde{X}_{n}\right) \underset{n \rightarrow \infty}{\longrightarrow} C(\tilde{Y})$.

Nótese que si $l\left(\tilde{Y}_{0}\right)=0$ entonces $\max \{(d-a),(c-b)\}=0$, y $C\left(\tilde{X}_{n}\right) \underset{n \rightarrow \infty}{\longrightarrow} 1$.

Seguridad del Experto. Por otro lado, también puede interesarnos la precisión con la que el experto ha expresado su juicio probabilístico. Por ejemplo, un experto que por medio de los métodos propuestos llega a un número como $(0.2,0.22,0.23,0.25)$ es mucho más preciso que un experto que asigna una probabilidad $(0,0,1,1)$. De hecho, este útimo experto está expresando que no sabe nada sobre la probabilidad del evento que se está cuestionando, mientras que el primer experto transmite mucha seguridad. Podemos medir la seguridad del experto en su juicio probabilístico mediante el área bajo su función de pertenencia:

Dado un número borroso trapezoidal normalizado $\tilde{A}=(a, b, c, d) \in[0,1]^{\mathcal{T F}}$ que denota el juicio probabilístico de un experto para un evento determinado. Llamaremos seguridad de $\tilde{A}$ a

$$
S(\tilde{A})=1-\frac{d-a+c-b}{2} .
$$

\section{Propiedades de la seguridad expresada por el experto:}

1. $S(\tilde{A}) \in[0,1], \forall \tilde{A} \in[0,1]^{\mathcal{T F}}$.

2. $S(\tilde{A})=1$ si y sólo si $\tilde{A}$ es un número real.

3. $S(\tilde{A})=0$ si y sólo si $\tilde{A}$ es el intervalo $[0,1]$.

4. $S$ es una función continua en $[0,1]^{\mathcal{T F}}$.

5. $\tilde{A} \subseteq \tilde{B} \Rightarrow S(\tilde{A}) \geq S(\tilde{B})$, donde $\subseteq$ es la relación de subsumción.

Demostración: Las tres primeras propiedades son triviales. 
La demostración de la cuarta propiedad es similar a la demostración de la continuidad de la consistencia, ya que, por definición, $S(\tilde{A})=1-\frac{d-a+c-b}{2}=1-\frac{l\left(\tilde{A}_{1}\right)+l\left(\tilde{A}_{0}\right)}{2}$, siendo $\tilde{A}_{\alpha}$ el $\alpha$-corte de $\tilde{A}$ para $\alpha=0,1$.

Demostremos la quinta propiedad:

Sean $\tilde{A}=\left(a_{1}, b_{1}, c_{1}, d_{1}\right)$ y $\tilde{B}=\left(a_{2}, b_{2}, c_{2}, d_{2}\right)$. Si $\tilde{A} \subseteq \tilde{B}$ entonces $a_{1} \leq a_{2}, b_{1} \leq b_{2}$, $c_{1} \geq c_{2}$ y $d_{1} \geq d_{2}$, por tanto $d_{1}-a_{1} \geq d_{2}-a_{2}$ y $c_{1}-b_{1} \geq c_{2}-b_{2}$. Luego, $S(\tilde{A}) \geq S(\tilde{B})$.

Calidad del juicio probabilístico asignado por el experto. Podemos medir la calidad del juicio probabilístico dado por el experto agregando las medidas anteriores de consistencia y seguridad del experto mediante la siguiente función:

$$
\psi(\tilde{A})=C(\tilde{A})^{1-\tau} \times S(\tilde{A})^{\tau}, \forall \tilde{A} \in T F[0,1],
$$

donde $\tau \in(0,1)$ permite ponderar la consistencia y la precisión con objeto de suavizar la función de calidad en situaciones complejas.

Propiedades de calidad del juicio probabilístico dado por el experto: La calidad del juicio probabilístico expresada por el experto verifica las siguientes propiedades:

1. $\psi(\tilde{A}) \in[0,1]$.

2. $\psi(\tilde{A})=1 \Longleftrightarrow \tilde{A}=(a, a, a, a) \in \mathbb{R}$

3. $\psi(\tilde{A})=0 \Longleftrightarrow \tilde{A}$ es triangular, o $\tilde{A}=(0,0,1,1)=[0,1]$.

4. $\psi$ es una función continua en $[0,1]^{\mathcal{T F}}$.

\section{Demostración.}

1. Se sigue de manera trivial de las propiedades de las medidas de consistencia y seguridad dadas anteriormente.

2. $\psi(\tilde{A})=1 \Longleftrightarrow C(\tilde{A})=1$ y $S(\tilde{A})=1 \Longleftrightarrow d-a+c-b=0, \frac{c-b}{d-a}=1 \Longleftrightarrow d-a=b-c \mathrm{y}$ $c-b=d-a$. Por tanto, $b-c=c-b=d-a \Longleftrightarrow b-c=d-a=0 \Longleftrightarrow a=b=c=d$, ya que $0 \leq a \leq b \leq c \leq d \leq 1$. 
3. $\psi(\tilde{A})=0 \Longleftrightarrow C(\tilde{A})=0$ o $S(\tilde{A})=0$. Por un lado, $C(\tilde{A})=0 \Longleftrightarrow c-b=0(\tilde{A}$ es triangular). Y, por otro, $S(\tilde{A})=0 \Longleftrightarrow d-a+c-b=2 \Longleftrightarrow d-a-1=1-c+b$ $\mathrm{y}$, puesto que $0 \leq a \leq b \leq c \leq d \leq 1$, se tiene que $d-a-1 \leq 0$ y $1-c+b \geq 0$, luego $d-a-1=1-c+b=0$, y por tanto, $d-a=c-b=1$, luego $\tilde{A}=(0,0,1,1)$.

4. $\psi$ es una función continua por ser el producto de dos funciones continuas en un espacio métrico completo.

La utilidad de la función de calidad dada es obvia. En un proceso de elicitación interactivo, si el experto ha dado un número borroso con una calidad por debajo de un cierto umbral determinado previamente por el analista, teniendo en cuenta las condiciones del problema, se puede discutir el resultado con el experto e incluso, se podría optar por desechar la información de este experto y consultar a otros. De esta manera, se puede filtrar la información suministrada por un conjunto de expertos en un proceso de elicitación colectivo como es el protocolo SRI ampliado a siete fases [42].

\subsection{Un experimento computacional}

En esta sección presentamos un experimento computacional que incluye una simulación de diálogos analista-experto para analizar los posibles sesgos asociados con la utilización del método de elicitación propuesto, es decir, los sesgos de representatividad y disponibilidad asociados con la formación del juicio probabilístico del experto, así como los sesgos de ajuste y anclaje derivados del diálogo.

En particular, analizaremos la consistencia de los números borrosos derivados del método propuesto sobre expertos sobreconfiados, neutrales e inseguros. Además, consideraremos la propensión del experto a trabajar con el método de las loterías o con el método de las apuestas. Finalmente, se analizará la calidad de la información extraída.

Para realizar este experimento, en primer lugar proponemos una función que representa la importancia subjetiva de cada evento en la experiencia del experto. Los parámetros de esta función modelizan la propensión del experto a cometer sesgos de disponibilidad (el experto tiende a sobrestimar la probabilidad de eventos que han ocurrido recientemente) o sesgos de representatividad (el experto tiende a sobrestimar la probabilidad de eventos que tienen un alto impacto). 
En la Sección 3.6.2 proponemos funciones para representar la probabilidad de indiferencia en la respuesta del experto, modelizando el sesgo de anclaje y ajuste que representa una mayor o menor sobreconfianza en la respuesta.

Los resultados del experimento simulado se valoran en la Sección 3.6.3 para diferentes expertos más o menos sesgados.

\subsubsection{Modelización de la importancia de la experiencia del ex- perto}

Consideremos una población de tamaño $N$ de ceros y unos. Se trata de determinar la proporción $0 / 1$ que representa la probabilidad de ocurrencia de un determinado suceso.

La experiencia del experto se puede representar por $k$ proporciones aproximadas $\left\{p_{t} \pm \varepsilon\right\}_{t=1}^{k}$, a las que llamaremos "hitos", procedentes de $k$ muestras $\left\{\theta_{t}\right\}_{t=1}^{k}$ de tamaño $n_{t}$ que simulan además un orden cronológico.

No todos los hitos tienen la misma importancia, ya que el experto seguirá las heurísticas señaladas de disponibilidad y representatividad, y se pueden generar sesgos en la asignación de probabilidades. La importancia de cada hito para el experto será una función que dependerá de la propensión del experto a cometer sesgos, de su memoria, y del impacto provocado por los hitos raros:

$$
I_{(\alpha, \beta, \gamma, \delta)}\left(p_{t}\right)=1+\alpha\left(\frac{t}{k}\right)^{\gamma}+\beta\left(\delta\left|p_{t}-\frac{\sum_{t=1}^{k} p_{t}}{k}\right|\right) .
$$

En esta función, $\frac{t}{k}$ representa el sesgo más claramente relacionado con la heurística de disponibilidad (el experto dará más importancia a los hitos que han ocurrido recientemente, y tiende a olvidar los antiguos), $\gamma \geq 1$ representa la memoria del experto, que decrece con $\gamma, \delta\left|p_{t}-\frac{\sum_{t=1}^{k} p_{t}}{k}\right|$ simula el sesgo más relacionado con la heurística de repesentatividad (el experto sobrevalora la probabilidad de sucesos raros que han ocurrido alguna vez, dependiendo del impacto que tengan). Precisamente, $\delta \geq 0$ es el impacto provocado por un hito que se distancia una unidad de longitud de la media de la experiencia. Por otro lado, $\alpha, \beta \in[0,1]$ son las propensiones a cometer cada uno de los sesgos anteriores. Si $\alpha=\beta=0$ entonces la importancia de cada hito es 1 , y el experto no comete sesgos.

Una vez que el experto ha asignado la importancia de cada hito, su juicio probabilístico 
estará en torno a la abscisa del centro geométrico de esta distribución:

$$
C=\frac{\sum_{t=1}^{k} p_{t} I_{(\alpha, \beta, \gamma, \delta)}\left(p_{t}\right)}{\sum_{t=1}^{k} I_{(\alpha, \beta, \gamma, \delta)}\left(p_{t}\right)}=\frac{\sum_{t=1}^{k} p_{t}\left[1+\alpha\left(\frac{t}{k}\right)^{\gamma}+\beta\left(\delta\left|p_{t}-\frac{\sum_{t=1}^{k} p_{t}}{k}\right|\right)\right]}{\sum_{t=1}^{k}\left[1+\alpha\left(\frac{t}{k}\right)^{\gamma}+\beta\left(\delta\left|p_{t}-\frac{\sum_{t=1}^{k} p_{t}}{k}\right|\right)\right]} .
$$

Nótese que si el experto no comete sesgos, su juicio probabilístico es la media de los hitos.

El experto ve de manera borrosa que la probabilidad de ocurrencia del evento es el valor $C$. En la segunda fase, se desarrolla el método propuesto para aproximar $C$ mediante un número borroso.

\subsubsection{Simulación de diálogos analista-experto}

Ahora, el experto tratará de expresar su juicio probabilístico aproximando el valor de $C$ en un diálogo interactivo con el analista.

A medida que el valor $p$, que se va ajustando en los métodos de loterías y apuestas, se acerca al centroide, aumenta la probabilidad de que el experto juzgue los dos juegos ofrecidos como indiferentes. Llamaremos a esta probabilidad, probabilidad de indiferencia del experto.

Modelizaremos la probabilidad de indiferencia del experto mediante una función que decrece con la distancia al centroide.

Sea $M=\max \{|C-1|,|C|\}$, entonces la probabilidad de indiferencia del experto se anula cuando $d(p, C)=M$, y vale 1 si $C=p$, es decir, si $d(p, C)=0$.

Si el experto tiene exceso de confianza, su probabilidad de indiferencia, como función de la distancia al centroide, decrecerá rápidamente al alejarnos del mismo, mientras que si desconfía de la experiencia, el decrecimiento será más suave. Si el experto no presenta un sesgo de confianza, entonces la probabilidad de indiferencia decrecerá de manera proporcional a la distancia al centroide (Figura 3.6.1).

El exceso de confianza se puede modelizar con una función $f(x)=e^{-\lambda x} \operatorname{con} \lambda>0$. La desconfianza, mediante funciones $f(x)=a-b e^{c x-d} \operatorname{con} a, b, c, d>0$. Finalmente, la neutralidad se puede modelizar con una recta $f(x)=1-(1 / M) x$, con $M=\max \{|C-1|,|C|\}$.

Durante el diálogo simulado también se debe modelizar la predisposición que tenga el experto a utilizar un método u otro (loterías o apuestas). El método más cómodo para el 
experto mejorará la confianza en su juicio. De este modo, la probabilidad de indiferencia del experto serán dos funciones con distintas pendientes en un método u otro.

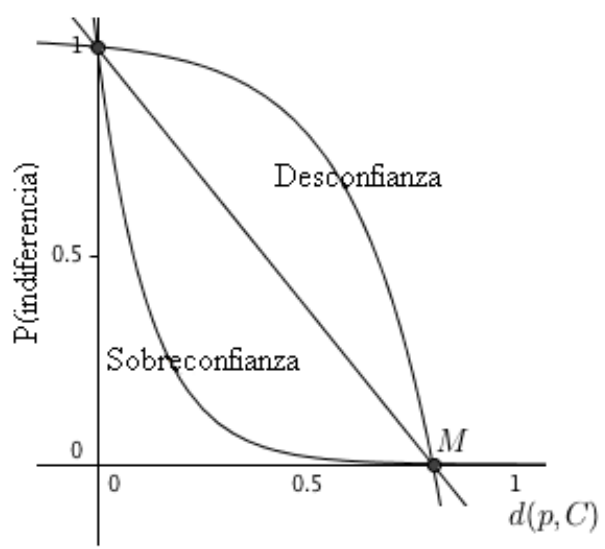

Figura 3.6.1: Funciones de probabilidad de indiferencia para modelizar el sesgo de confianza

El pseudocódigo empleado en la simulación para el método propuesto es:

a) Método de Loterías (Intervalar).

- Paso 0: Fijar los valores monetarios $x$ e $y$. Fijar un valor $p_{0} \in[0,1]$

- Paso $i+1$ : Generar un número aleatorio $n$ en $[0,1]$.

- Mientras $n>f\left(\left|p_{i}-C\right|\right)$ (no hay indiferencia) entonces

○ Si $p_{i}<C$, entonces tomar $p_{i+1}>p_{i}$ y repetir.

○ Si $p_{i}>C$, entonces tomar $p_{i+1}<p_{i}$ y repetir.

- (En caso contrario hay indiferencia). Poner $p=p_{i}$

- Generar $p_{i}^{-} \in\left(p_{i}-\varepsilon, p_{i}\right)$ y generar un número aleatorio $n$ en $[0,1]$.

$\diamond$ Si $n>f\left(\left|p_{i}^{-}-C\right|\right)$, guardar $p_{\text {min }}^{L}=p_{i}$.

$\diamond$ En caso contrario, hacer $p_{i}=p_{i}^{-}$y repetir.

○ Hacer $p_{i}=p$.

- Generar $p_{i}^{+} \in\left(p_{i}, p_{i}+\varepsilon\right)$ y generar un número aleatorio $n$ en $[0,1]$.

$\diamond$ Si $n>f\left(\left|p_{i}^{+}-C\right|\right)$ guardar $p_{\max }^{L}=p_{i}$

$\diamond$ En caso contrario, hacer $p_{i}=p_{i}^{+}$y repetir .

- Proporcionar el intervalo $\left[p_{\min }^{L}, p_{\max }^{L}\right]$. 
b) Método de las Apuestas (Intervalar).

- Paso 1. Fijar los valores monetarios $x_{0}$ e $y_{0}$.

- Paso $i+1$ : Generar un número aleatorio $n$ en $[0,1]$.

- Mientras $n>g\left(\left|\frac{y_{i}}{x_{i}+y_{i}}-C\right|\right)$ (no hay indiferencia) entonces

- Si $\frac{y_{i}}{x_{i}+y_{i}}<C$ entonces tomar $x_{i+1}, y_{i+1}$ tal que $\frac{y_{i+1}}{x_{i+1}+y_{i+1}}>\frac{y_{i}}{x_{i}+y_{i}}$ y repetir.

○ $\mathrm{Si} \frac{y_{i}}{x_{i}+y_{i}}>C$ entonces tomar $x_{i+1}, y_{i+1}$ tal que $\frac{y_{i+1}}{x_{i+1}+y_{i+1}}<\frac{y_{i}}{x_{i}+y_{i}}$ y repetir.

- (En caso contrario hay indiferencia). Hacer $x=x_{i}, y=y_{i}$.

○ Generar $x_{i}^{-}, y_{i}^{-}$tal que $\frac{y_{i}^{-}}{x_{i}^{-}+y_{i}^{-}} \in\left(\frac{y_{i}}{x_{i}+y_{i}}-\varepsilon, \frac{y_{i}}{x_{i}+y_{i}}\right)$ y generar un número aleatorio $n$ en $[0,1]$.

$\diamond$ Si $n>g\left(\left|\frac{y_{i}^{-}}{x_{i}^{-}+y_{i}^{-}}-C\right|\right)$ guardar $p_{\min }^{A}=\frac{y_{i}}{x_{i}+y_{i}}$

$\diamond$ En caso contrario, hacer $x_{i}=x_{i}^{-}$e $y_{i}=y_{i}^{-}$y repetir.

○ Hacer $x_{i}=x, y_{i}=y$

○ Generar $x_{i}^{+}, y_{i}^{+}$tal que $\frac{y_{i}^{+}}{x_{i}^{+}+y_{i}^{+}} \in\left(\frac{y_{i}}{x_{i}+y_{i}}, \frac{y_{i}}{x_{i}+y_{i}}+\varepsilon\right)$ y generar un número aleatorio $n$ en $[0,1]$.

$\diamond$ Si $n>g\left(\left|\frac{y_{i}^{+}}{x_{i}^{+}+y_{i}^{+}}-C\right|\right)$ guardar $p_{\text {max }}^{A}=\frac{y_{i}}{x_{i}+y_{i}}$

$\diamond$ En caso contrario, hacer $x_{i}=x_{i}^{-}$e $y_{i}=y_{i}^{-}$y repetir .

- Proporcionar el intervalo $\left[p_{\min }^{A}, p_{\max }^{A}\right]$.

\subsubsection{Experimento}

En primer lugar generamos con el software estadístico $R$ project una población binaria de $10^{6}$ elementos en la proporción 0.3 , que representan la realidad.

Representaremos la experiencia del experto por 10 muestras aleatorias de distintos tamaños tomadas de esta población y calculamos la proporción de cada muestra. A cada una de las 10 proporciones la llamaremos hito en la experiencia del experto.

La Tabla 3.6.1 muestra estas proporciones y la importancia que el experto asigna a cada hito según diferentes propensiones a cometer sesgos de representatividad y disponibilidad y con parámetros $\gamma=5$ y $\delta=10$. 
Tabla 3.6.1: Hitos de experiencia de expertos e importancia asignada según su propensión al sesgo

\begin{tabular}{cccccccccccc}
\hline Muestra & 1 & 2 & 3 & 4 & 5 & 6 & 7 & 8 & 9 & 10 & \\
\hline \hline$p_{t}$ & 0.27 & 0.3 & 0.4 & 0.32 & 0.26 & 0.3 & 0.34 & 0.43 & 0.28 & 0.22 & $C$ \\
\hline$I_{(1,1,5,10)}\left(p_{t}\right)$ & 1.42 & 1.12 & 1.88 & 1.09 & 1.55 & 1.19 & 1.45 & 2.51 & 1.91 & 2.92 & 0.31 \\
\hline$I_{(0,0,5,10)}\left(p_{t}\right)$ & 1.00 & 1.00 & 1.00 & 1.00 & 1.00 & 1.00 & 1.00 & 1.00 & 1.00 & 1.00 & 0.31 \\
\hline$I_{(1,0,5,10)}\left(p_{t}\right)$ & 1.00 & 1.00 & 1.00 & 1.01 & 1.03 & 1.08 & 1.17 & 1.33 & 1.59 & 2.00 & 0.30 \\
\hline$I_{(0,1,5,10)}\left(p_{t}\right)$ & 1.42 & 1.12 & 1.88 & 1.08 & 1.52 & 1.12 & 1.28 & 2.18 & 1.32 & 1.92 & 0.31 \\
\hline
\end{tabular}

Tabla 3.6.2: Centroides con el suceso raro $p_{10}=0.8$ según propensión al sesgo

\begin{tabular}{cc}
\hline Propensión al sesgo $(\alpha, \beta)$ & Centroide para $p_{10}=0.8$ \\
\hline \hline Experto sesgado $(1,1)$ & 0.45 \\
\hline Experto insesgado $(0,0)$ & 0.37 \\
\hline Sesgo de disponibilidad $(1,0)$ & 0.40 \\
\hline Sesgo de representatividad $(0,1)$ & 0.44 \\
\hline
\end{tabular}

Vemos que la influencia de los sesgos de representatividad y disponibilidad no es significativa puesto que los centroides son prácticamente iguales (0.30 y 0.31). Esto se debe a la inexistencia de sucesos raros en la experiencia del experto. De este modo, para analizar la influencia de los sesgos de representatividad y disponibilidad, necesitamos introducir un evento raro.

Supongamos entonces que el décimo hito en la experiencia del experto es un evento raro con $p_{10}=0.8$, entonces el centroide resulta 0.45 si el experto es propenso a cometer sesgos $(\alpha=\beta=1)$. En la Tabla 3.6.2 se muestran los nuevos centroides incluyendo el evento raro para diferentes situaciones de propensión al sesgo. Cuando el experto es insesgado, el centroide coincide con la media 0.37. Analizaremos ambos casos.

A continuación, simulamos los diálogos analista-experto para analizar los posibles sesgos asociados con el uso del método de elicitación probabilístico. Consideraremos tres situaciones correspondientes a un experto confiado, inseguro (desconfiado en su experiencia) o neutral.

\section{Experto confiado}

Consideremos un experto confiado modelizado por sus funciones de probabilidad de indiferencia $f(x)=e^{-4 x}$ y $f(x)=e^{-6 x}$ para los métodos de loterías y apuestas respectivamente, es decir, asumimos que el experto prefiere las apuestas a las loterías.

Además, diferenciamos al experto confiado sesgado (con $\alpha=\beta=1)$ del experto con- 
fiado insesgado $(\alpha=\beta=0)$ en función de su propensión a cometer sesgos.

Generamos 100 diálogos con el mismo experto para cada método. Suponemos que el experto no aprende de cada diálogo, es decir, que los diálogos son independientes.

Lo primero que apreciamos es que, como era de esperar, el primer valor que propone el analista en cada método ancla el resto del diálogo y las respuestas del experto. En la Figura 3.6.2 se pueden ver las respuestas del experto al método nítido de las loterías para aproximar el valor $C=0.45$ (correspondiente al experto sesgado) a partir de dos valores de anclaje diferentes. La línea discontinua corresponde a un valor de $p=0.9$, a partir del cual el analista comienza a descender hasta obtener el valor de indiferencia, y la línea continua corresponde a un valor inicial de $p=0.1$ a partir del cual el analista asciende hasta encontrar el valor de indiferencia. La línea horizontal de puntos marca el valor de $C=0.45$. Como puede verse, en la mayoría de las ocasiones el experto da un valor por encima o por debajo de $C$ dependiendo del valor inicial (el ancla) propuesto por el analista.

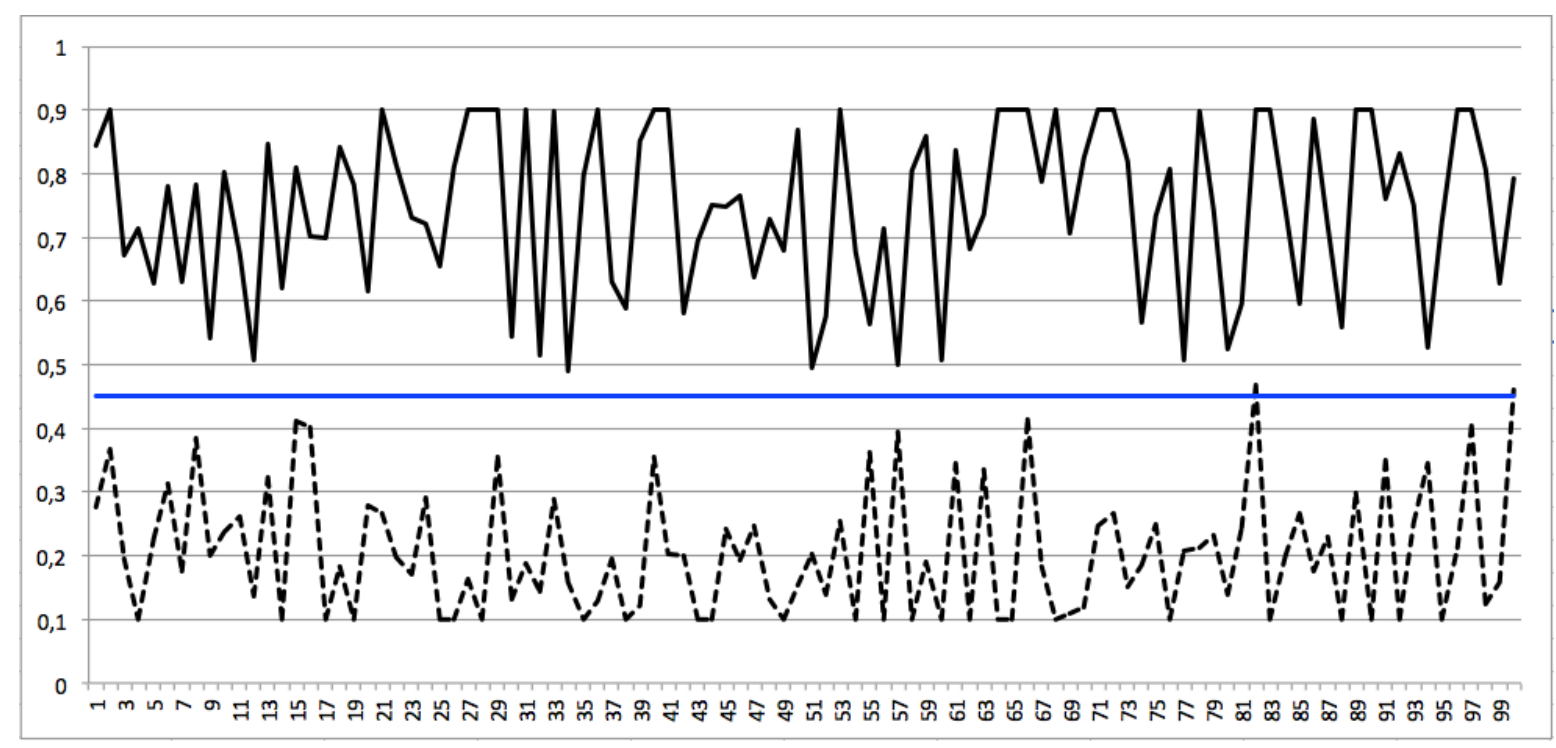

Figura 3.6.2: Método clásico de loterías para los anclas $p=0.1$ y 0.9 

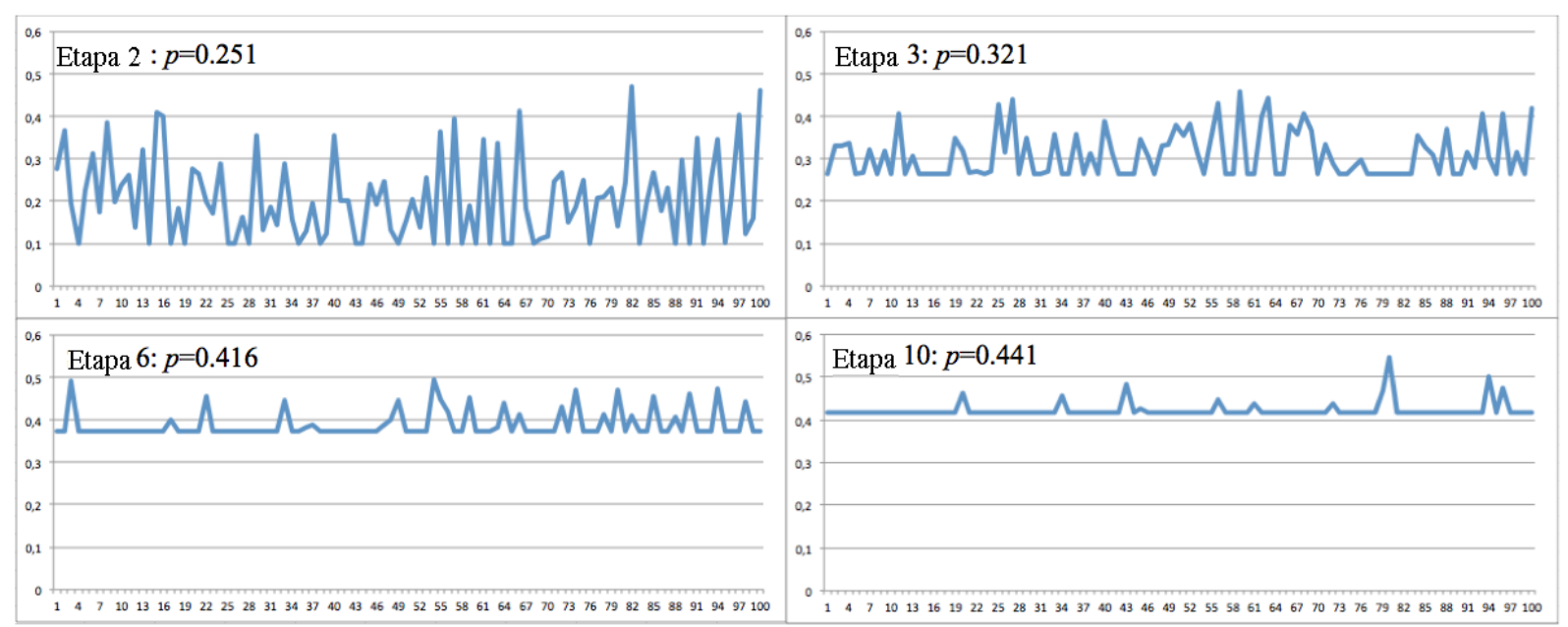

Figura 3.6.3: Aproximación del ancla para un experto confiado

Esta circunstancia nos da una oportunidad para mejorar el método que consiste en refinar progresivamente los resultados. Si realmente el analista pudiera realizar 100 diálogos independientes con el mismo experto, entonces el analista podría calcular el valor medio de las respuestas obtenidas y anclar otros 100 diálogos en dicho valor, hasta conseguir eliminar la variabilidad del experto. Este proceso ideal (utópico) puede verse en la Figura 3.6.3.

En cada etapa, el analista ancla el diálogo en el valor medio obtenido del experto en los 100 diálogos de la etapa anterior. Esto lleva a aproximar con mucha fiabilidad el juicio probabilístico del experto.

Obviamente, en la realidad este proceso es imposible, pero sí se puede realizar un proceso iterativo en el que los métodos de apuestas y loterías se retroalimenten hasta converger. Así por ejemplo, el analista, anclando un primer diálogo con el método de las loterías en $p_{0}$, obtendrá del experto un nuevo valor de $p_{1}$ que le servirá (al analista) para anclar un diálogo con el método de las apuestas del que obtendrá otro valor $p_{2}$. Entonces, pasado un tiempo razonable, el analista usará $p_{2}$ para anclar un nuevo diálogo con el método de las loterías. Cuando las diferencias entre $p_{i}$ y $p_{i+1}$ dejen de ser significativas, comenzará el método borroso partiendo del ancla adecuado.

En la Figura 3.6.5 pueden verse los resultados intervalares obtenidos por ambos métodos tras un refinamiento previo para anclar el diálogo en $p=0.445$. El refinamiento puede verse en la Figura 3.6.4. Partiendo de $p_{0}=0.1$ se desarrolla el método (nítido) de 

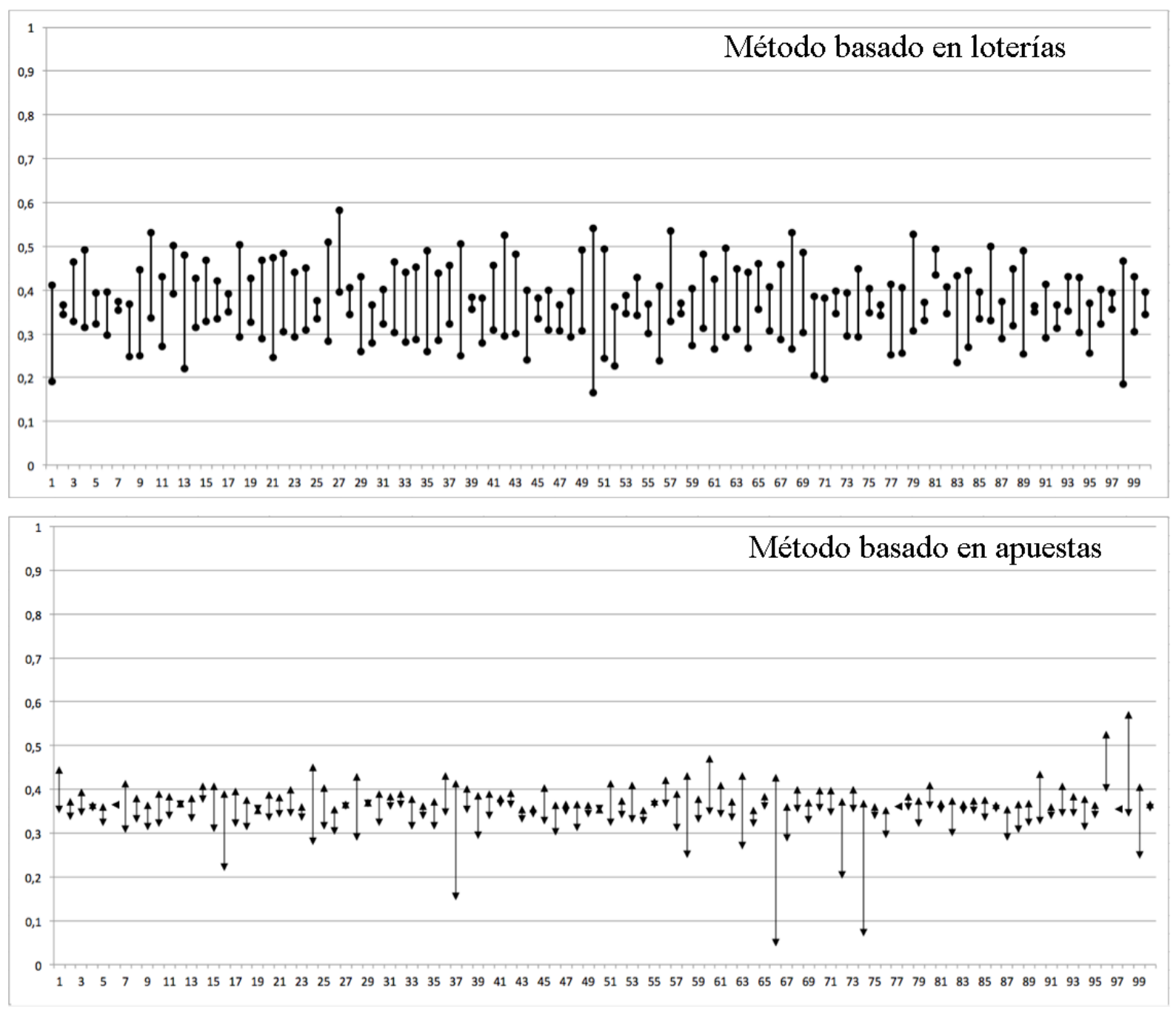

Figura 3.6.4: Intervalos obtenidos por cada método para el experto sesgado confiado

las loterías y se obtiene $p_{1}=0.25$. Este valor es el ancla para el método (nítido) de las apuestas, del que se obtiene $p_{2}=0.35$. A su vez, este valor se utiliza como ancla de nuevo para el método de las loterías, y se obtiene $p_{3}=0.4$. Así sucesivamente hasta encontrar la convergencia en $p=0.445$.

En la Figura 3.6.4 pueden verse los cien números borrosos que resultan de mezclar los pares de intervalos obtenidos en las simulaciones de los cien diálogos.

Los rombos de la Figura 3.6.5 representan la calidad de la información obtenida una vez ajustado el parámetro $\tau$ de la Ecuación 3.5.1. Un intervalo $\widetilde{A}$ de longitud 0.15 (y consistencia igual a 1) tiene una precisión asociada de 0.9 , entonces $C(\widetilde{A})=(1-0.15)^{\tau}=$ 0.9 , de donde $\tau=0.6270477$. 

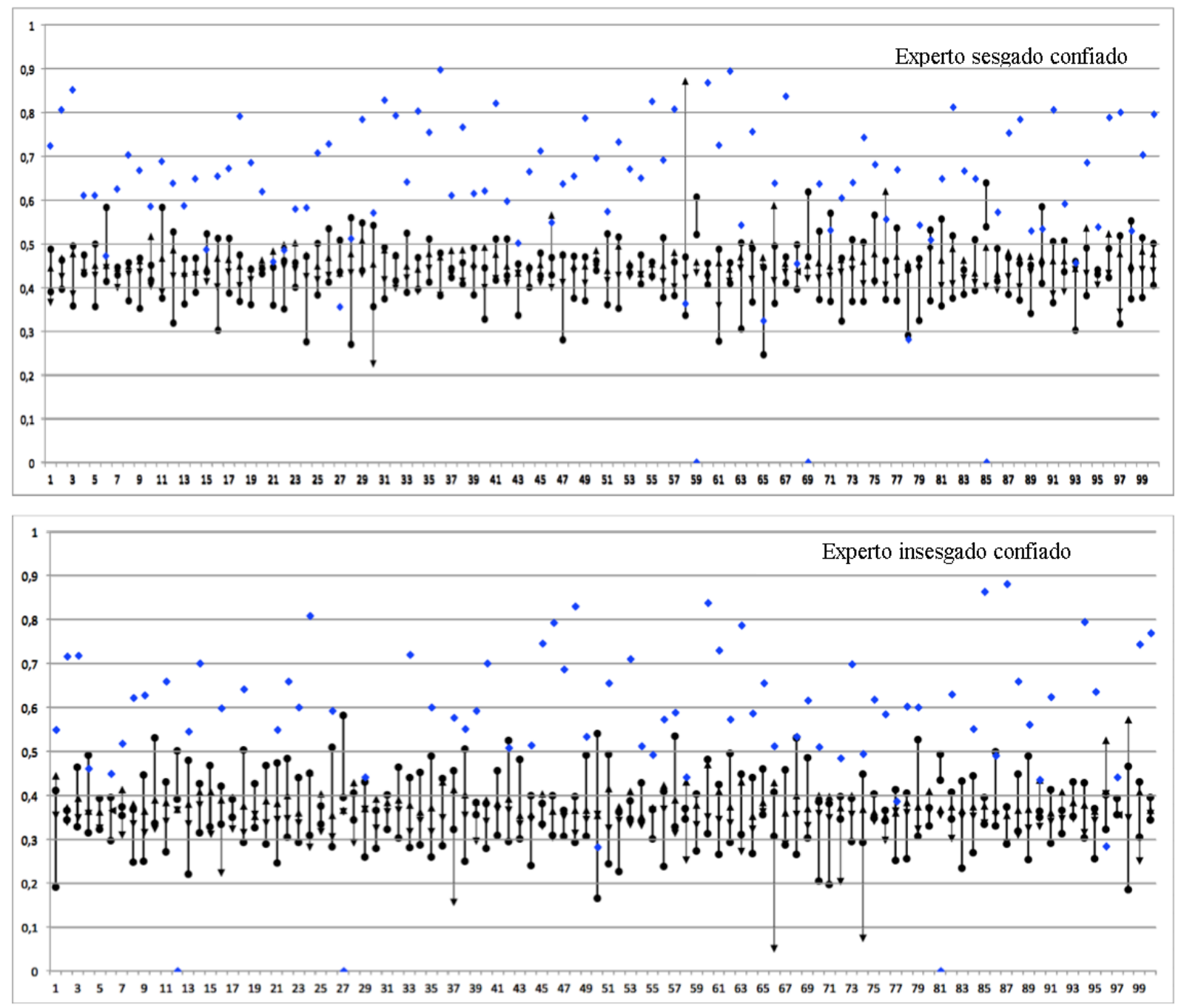

Figura 3.6.5: Números borrosos y su calidad para el experto confiado (sesgado e insesgado)

La calidad de cada número borroso elicitado es muy sensible a la amplitud de dicho número. Nótese además que la inconsistencia de los intervalos obtenidos lleva en tres casos a una calidad nula.

En la Tabla 3.6.3 puede verse un conjunto de parámetros estadísticos que resume los resultados obtenidos para el experto confiado sesgado $(\alpha=\beta=1)$ y para el experto confiado insesgado $(\alpha=\beta=0)$ para el que se ha desarrollado un proceso similar, tras el refinamiento del valor de anclaje.

Nótese que tanto para el experto sesgado como para el insesgado todos los números borrosos trapezoidales incluyen los valores $C=0.45$ y $C=0.37$, respectivamente, y particularmente, con posibilidad 1 en el $74 \%$ y $72 \%$ de los casos y muy cercanos a 1 en el resto de casos. 
La calidad de la información obtenida es sólo ligeramente mayor para el experto sesgado.

En conclusión, los resultados para el experto confiado son consistentes con los métodos clásicos de elicitación, al contener generalmente el número borroso al centroide que determina el juicio probabilístico del experto. Pero además, el proceso de refinamiento que podemos desarrollar al usar secuencialmente los métodos de apuestas y loterías nos permite identificar un ancla adecuado para iniciar la elicitación del juicio probabilístico.

Tabla 3.6.3: Estadísticas sobre la calidad de los resultados para el experto confiado (sesgado e insesgado)

\begin{tabular}{ccccc}
\hline & $\begin{array}{c}\text { Sesgado } \\
\text { Media }\end{array}$ & Desviación est. & $\begin{array}{c}\text { Insesgado } \\
\text { Media }\end{array}$ & Desviación est. \\
\hline \hline Método Loterías. & {$[0.382,0.494]$} & $0.0479,0.0423$ & {$[0.299,0.435]$} & $0.0459,0.0505$ \\
\hline Método Apuestas. & {$[0.409,0.481]$} & $0.0284,0.0503$ & {$[0.312,0.396]$} & $0.0520,0.0337$ \\
\hline Calidad & 0.631 & 0.1668 & 0.593 & 0.1563 \\
\hline
\end{tabular}

\section{Inseguridad}

Ahora asumamos que la probabilidad de indiferencia del experto es $g_{1}(x)=1,1-$ $e^{\frac{\ln (11)}{0,55} x+\ln (0,1)}$ y $g_{2}(x)=1-e^{10(x-0.47)+\ln (0,5)}$ (Figura 3.6.6), que denota una inseguridad pronunciada del experto sobre su experiencia. Nos referiremos a este experto como experto inseguro. Además, al igual que en el caso anterior, diferenciaremos al experto sesgado $(\alpha=\beta=1)$ del experto insesgado $(\alpha=\beta=0)$.

Un experto inseguro se asemeja a una situación de desconocimiento del experto. Cuanto más pronunciada es la inseguridad (las funciones de probabilidad de indiferencia) menos confiable es la experiencia del experto, el cual responderá rápidamente con la indiferencia a las preguntas del analista, lo que dificulta superar valores cercanos al ancla inicial propuesto por el analista. Es decir, el proceso de refinamiento lleva a resultados no convenientes, como puede verse en la Figura 3.6.7.

Si consideramos un experto inseguro sesgado $(\alpha=\beta=1$ y $C=0.45$, ver Tabla 3.6.2) la variabilidad se va reduciendo muy lentamente a partir del ancla inicial $p_{1}=0.1 \mathrm{y}$ los resultados de etapas consecutivas son insignificantes desde el principio. Esto dificultará 
el proceso de elicitación y, como puede verse en la Etapa 19, el resultado puede ser muy lejano de la realidad.

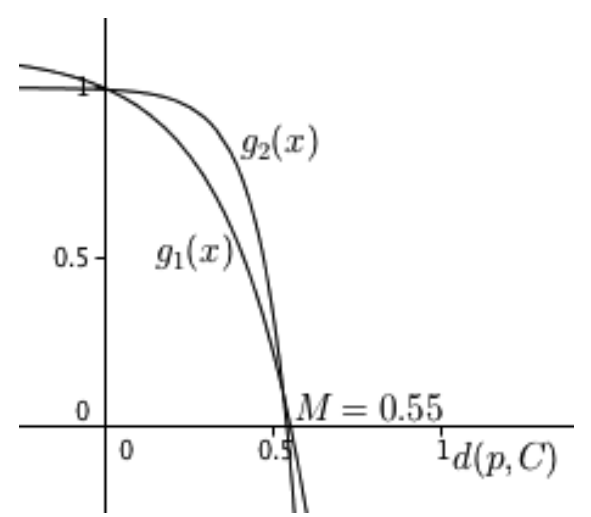

Figura 3.6.6: Probabilidad de indiferencia en la respuesta de un experto desconfiado

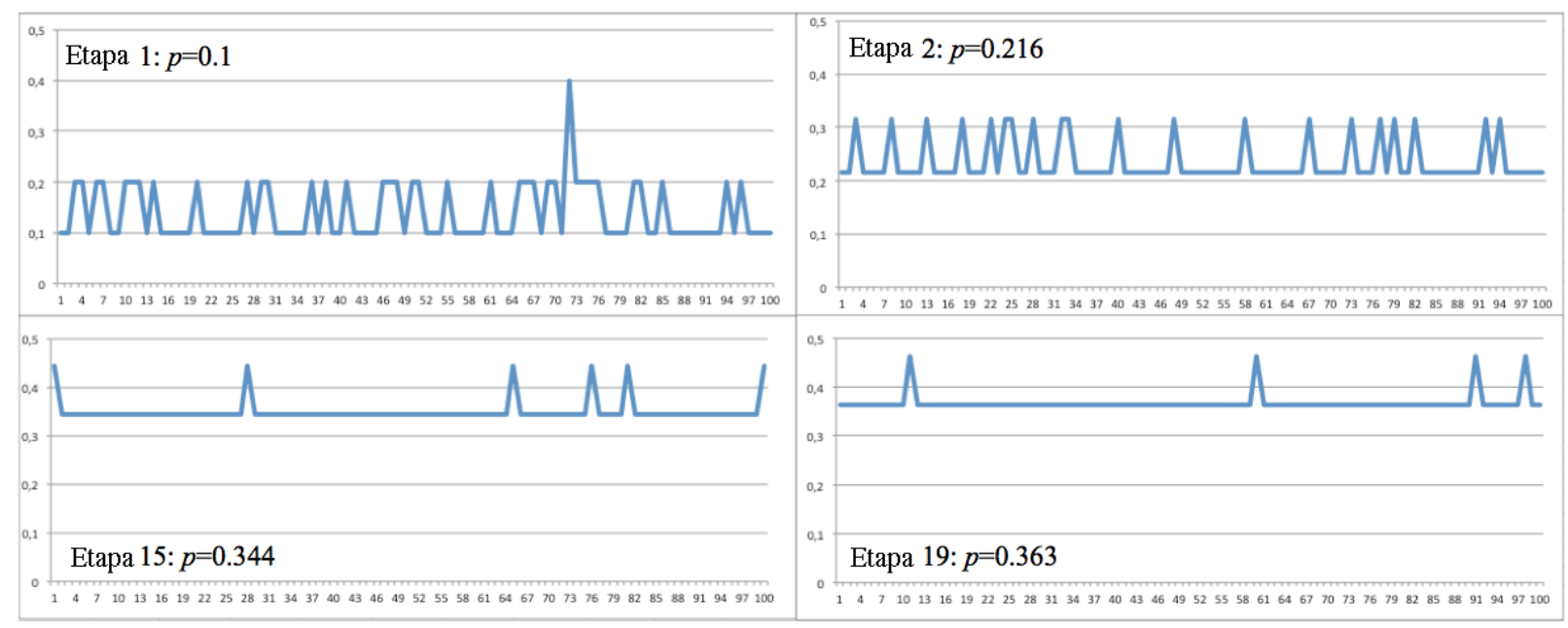

Figura 3.6.7: Proceso de aproximación del ancla apropiado para el experto desconfiado.

Con los métodos clásicos de elicitación el analista no puede ver cuándo el experto es inseguro. Por tanto, en esta situación la única opción es que el experto declare honestamente su dificultad para asignar el juicio probabilístico que se está elicitando.

En la Figura 3.6.8 puede verse el resultado de cien diálogos analista-experto simulados a partir del ancla $p=0.2$. Todos los resultados son completamente erróneos, y en la mayoría de los casos el experto es inconsistente. El experto inseguro insesgado lleva igualmente a resultados incorrectos dada la influencia del ancla sobre el desarrollo del proceso de elicitación. 


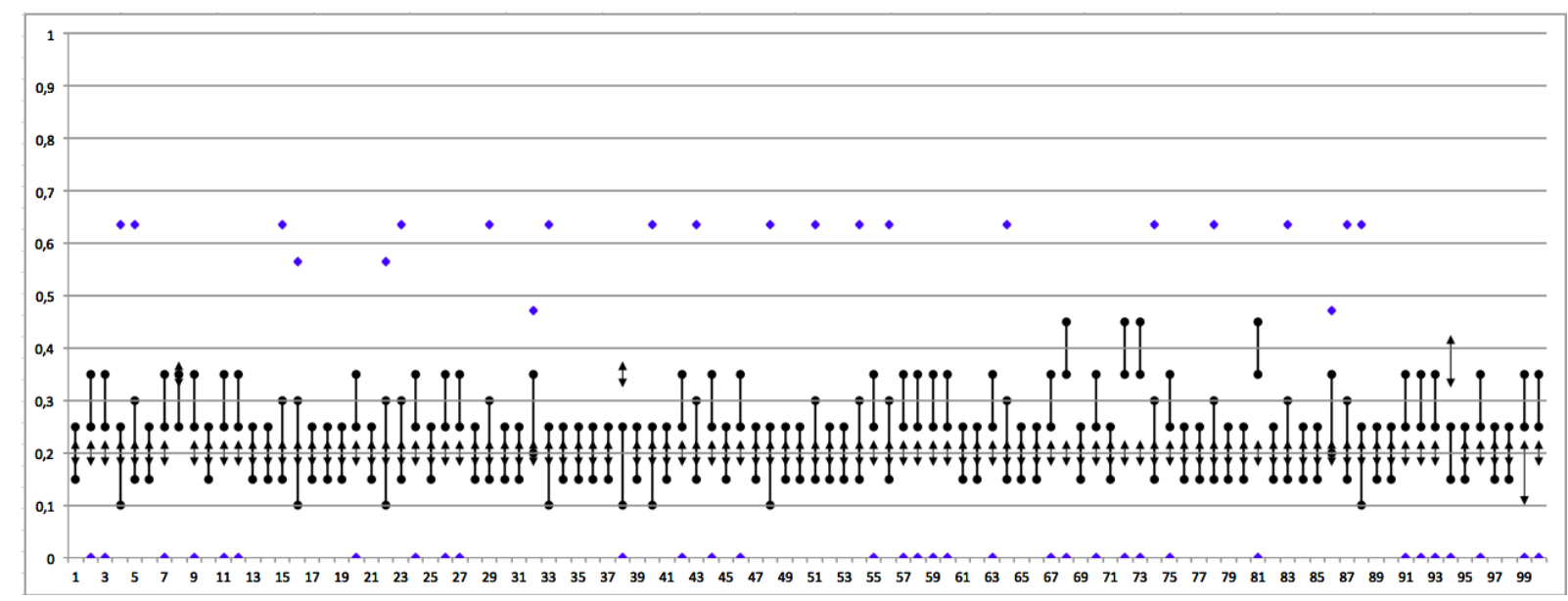

Figura 3.6.8: Resultados para el experto desconfiado a partir de un ancla $p=0.2$

\section{Neutralidad}

A continuación, consideramos el experto neutral con funciones de probabilidad de indiferencia $f(x)=-\frac{1}{0.63} x+1$ y $f(x)=e^{-4 x}$ asumiendo que el experto prefiere el método de las loterías al método de las apuestas.

En la Figura 3.6.9 podemos ver los resultados de los expertos neutral sesgado y neutral insesgado. Sus estadísticos asociados se muestran en la Tabla 3.6.4.

A diferencia del experto confiado, el número borroso trapezoidal incluye a los centroides $C=0.45$ y $C=0.37$ sólo en el $87 \%$ y $79 \%$ de los casos, y con posibilidad 1 , únicamente en el $33 \%$ y el $16 \%$, respectivamente.

Observamos que la calidad media (y su dispersión) de los números borrosos trapezoidales derivados son similares para los expertos neutrales sesgado e insesgado. Nótese que se obtienen más resultados inconsistentes (de calidad nula) para el experto neutral que para el experto confiado. Además, vemos que la dispersión de la calidad es mayor para el experto neutral.

Podemos concluir que los resultados de usar el método de elicitación de probabilidades propuesto es consistente con los métodos clásicos de elicitación para un experto neutral sesgado o insesgado. Como era de esperar, los resultados son peores que los obtenidos para el experto confiado en términos de las posibilidades asociadas con los centroides 0.45 y 0.37 en los números borrosos obtenidos para el experto sesgado e insesgado, respectivamente. Además, la calidad media de dichos resultados es menor que en el caso del experto 
confiado, mientras que su dispersión es mayor.
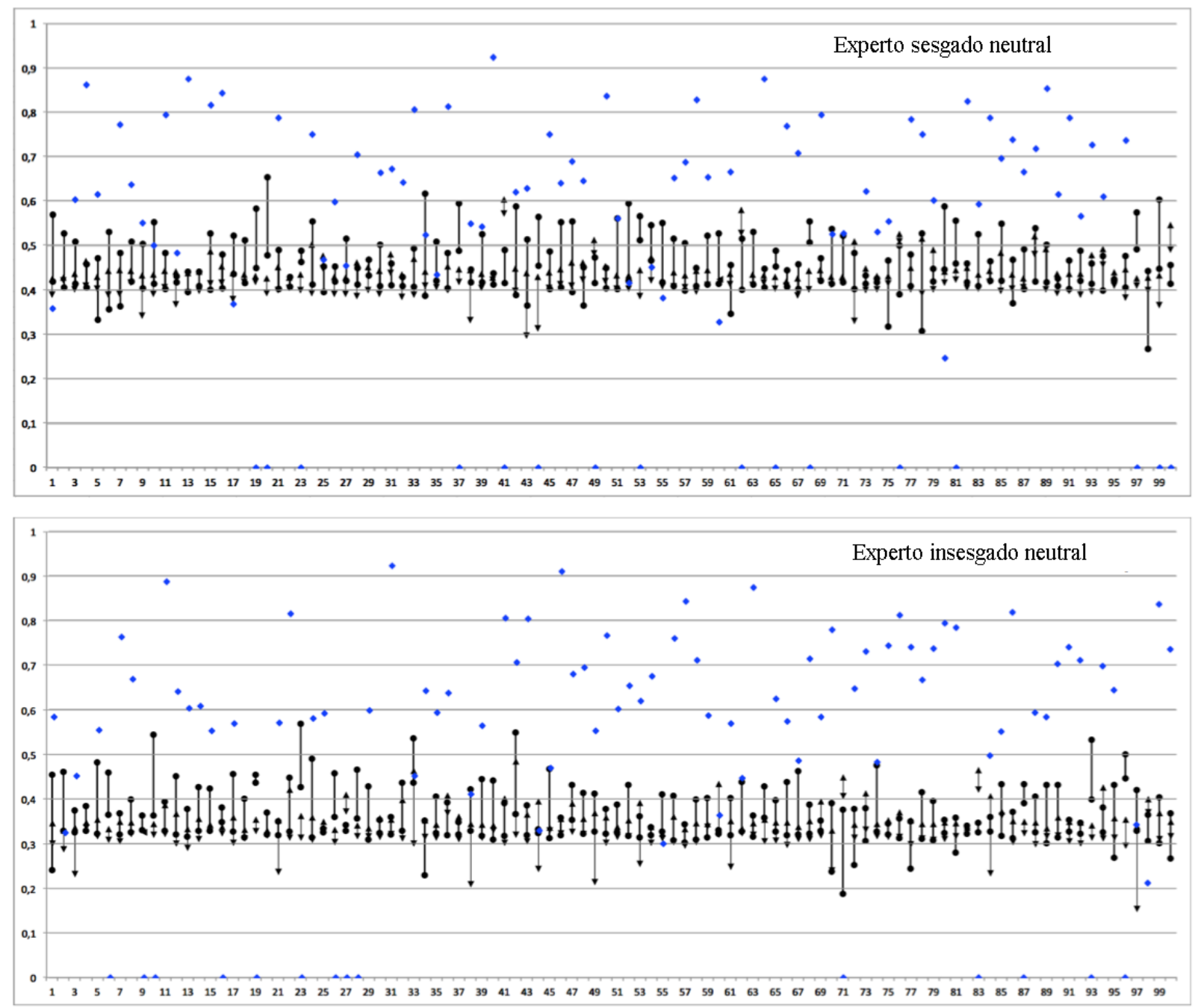

Figura 3.6.9: Resultado para el experto neutral

Tabla 3.6.4: Estadísticas para el experto neutral

\begin{tabular}{ccccc}
\hline & $\begin{array}{c}\text { Sesgado } \\
\text { Media }\end{array}$ & Desviación est. & $\begin{array}{c}\text { Insesgado } \\
\text { Media }\end{array}$ & Desviación est. \\
\hline \hline Método Loterías. & {$[0.401,0.499]$} & $0.0346,0.0497$ & {$[0.322,0.406]$} & $0.0371,0.0519$ \\
\hline Método Apuestas. & {$[0.398,0.458]$} & $0.0356,0.0324$ & {$[0.301,0.368]$} & $0.0350,0.0317$ \\
\hline Calidad & 0.5426 & 0.27 & 0.5468 & 0.261 \\
\hline
\end{tabular}




\section{Capítulo 4 SIMILITUD DE NÚMEROS BORROSOS TRAPEZOIDALES GENERALIZADOS}

Como hemos visto en el Capítulo 3, cuando se hace uso de la Lógica Borrosa para modelizar y tratar la información que aportan los expertos (modelado lingüístico borroso) se debe definir un modelo de representación y un modelo computacional.

El mayor problema del modelo de representación consiste en la elección de escalas de términos lingüísticos adecuadas para todos los expertos. Sin embargo, el establecimiento de estas escalas puede llevar a sesgos derivados de la discretización de los valores entre los que debe decidir el experto y la cantidad de términos lingüísticos puede ser una cuestión controvertida. Este problema se puede resolver en el caso probabilístico mediante la metodología de educción de probabilidades vista en la Sección 3.3, pero esta metodología tiene en su contra la cantidad de tiempo y recursos necesarios para desarrollar los diálogos analista-experto. Una asignación directa es mucho más rápida, pero puede llevar a cometer errores importantes.

Tanto si utilizamos una asignación directa como un método interactivo, la información aportada por los expertos son las entradas del modelo computacional. Las dificultades en el establecimiento de este modelo consisten en utilizar una aritmética de números borrosos adecuada (lo veremos con detalle en el Capítulo 5) y en trasladar los resultados de los operadores de este modelo al modelo de representación. Recordemos (Sección 3.3) que los resultados de las operaciones aritméticas entre los números borrosos de una escala de términos lingüísticos en general no es un elemento de esta escala, de modo que para poder informar de tales resultados hemos de asignar convenientemente un término lingüístico de la escala al resultado. De hecho, asignaremos el término más parecido. 
Para establecer la similitud entre los números borrosos implicados se puede recurrir a diferentes parámetros que dan una idea de la distancia entre ambos, de la forma o del tamaño. Estos parámetros se agregan en expresiones que definen el grado de similitud entre dos números borrosos.

Recordemos del Capítulo 3 que un número borroso trapezoidal generalizado con soporte en el intervalo $[0,1]$ de la recta real es una tupla $\left(a_{1}, a_{2}, a_{3}, a_{4} ; w_{\widetilde{A}}\right)$ con $0 \leq a_{1} \leq$ $a_{2} \leq a_{3} \leq a_{4} \leq 1, w_{\widetilde{A}} \in(0,1]$ junto con una función de pertenencia trapezoidal con vértices $\left(a_{1}, 0\right),\left(a_{2}, 1\right),\left(a_{3}, 1\right)$ y $\left(a_{4}, 0\right)$, y con altura $w_{\widetilde{A}}[9]$.

Los números borrosos trapezoidales generalizados sobre la recta real $\left(\mathbb{R}^{\mathcal{T} \mathcal{F} G}\right)$ han sido utilizados en numerosos problemas en ambiente borroso, como problemas de transporte borroso [51], problemas de flujo máximo y otros problemas de programación lineal [34].

Este capítulo repasa el estado del arte de las funciones de similitud de números borrosos trapezoidales generalizados, desde las primeras funciones utilizadas en la literatura hasta las funciones actuales. Veremos las propiedades deseables que debe tener una función de similitud de números borrosos trapezoidales así como las ventajas y los inconvenientes de las funciones de la literatura específica en este campo.

\subsection{Funciones de similitud de números borrosos trape- zoidales}

Una función de similitud de números borrosos trapezoidales generalizados con soporte en $[0,1]$ es una función $S:[0,1]^{\mathcal{T F} G} \times[0,1]^{\mathcal{T F} G} \longrightarrow[0,1]$ que indica el grado de semejanza entre dos números borrosos. Este valor debe coincidir con la percepción intuitiva que tenemos de los números que se están comparando. Cuanto más próximo esté este valor a 1, tanto más parecidos serán los números borrosos comparados.

Por ejemplo, una de las primeras medidas de similitud, utilizada por Chen [10] en 1996, determina el grado de similitud entre los números borrosos $\widetilde{A}=\left(a_{1}, a_{2}, a_{3}, a_{4} ; 1\right)$ y $\widetilde{B}=\left(b_{1}, b_{2}, b_{3}, b_{4} ; 1\right)$, utilizando el concepto de distancia geométrica [10]

$$
S(\widetilde{A}, \widetilde{B})=1-\frac{\sum_{i=1}^{4}\left|a_{i}-b_{i}\right|}{4} .
$$

Esta medida tiene una serie de propiedades interesantes para asignar la similitud entre dos números trapezoidales normalizados: 
- Propiedad 1: $S(\widetilde{A}, \widetilde{B})=S(\widetilde{B}, \widetilde{A})$.

- Propiedad 2: $S(\widetilde{A}, \widetilde{B})=1 \Longleftrightarrow \widetilde{A}=\widetilde{B}$.

- Propiedad 3: Si $\widetilde{A}=(a, a, a, a ; 1)$ y $\widetilde{B}=(b, b, b, b ; 1)$, entonces

$$
S(\widetilde{A}, \widetilde{B})=1-|a-b|
$$

Sin embargo, esta medida no sirve para medir la similitud entre números borrosos generalizados.

El propio Chen [11] extendió su medida de similitud en 2003 al conjunto $T F G[0,1]$ añadiendo a la expresión anterior la distancia entre los centros de gravedad de los números comparados. La medida de similitud entre los números $\widetilde{A}=\left(a_{1}, a_{2}, a_{3}, a_{4} ; w_{\widetilde{A}}\right)$ y $\widetilde{B}=$ $\left(b_{1}, b_{2}, b_{3}, b_{4} ; w_{\widetilde{B}}\right)$ estaría determinada por la expresión:

$$
S(\widetilde{A}, \widetilde{B})=\left[1-\frac{\sum_{i=1}^{4}\left|a_{i}-b_{i}\right|}{4}\right] \times\left[1-X_{\widetilde{A}}-X_{\widetilde{B}}\right]^{B\left(S_{\widetilde{A}}, S_{\widetilde{B}}\right)} \times\left[\frac{\min \left\{Y_{\widetilde{A}}, Y_{\widetilde{B}}\right\}}{\max \left\{Y_{\widetilde{A}}, Y_{\widetilde{B}}\right\}}\right]
$$

donde

$\left(X_{\widetilde{A}}, Y_{\widetilde{A}}\right)$ y $\left(X_{\widetilde{B}}, Y_{\widetilde{B}}\right)$ son los centroides de $\widetilde{A}$ y $\widetilde{B}$, respectivamente, es decir,

$$
\begin{gathered}
X_{\widetilde{A}}=\left\{\begin{array}{cr}
\frac{Y_{\widetilde{A}}\left(a_{3}+a_{2}\right)+\left(w_{\widetilde{A}}-Y_{\widetilde{A}}\right)\left(a_{4}+a_{1}\right)}{2 w_{\widetilde{A}}}, & \text { si } w_{\widetilde{A}} \neq 0 \\
Y_{\widetilde{A}}=\left\{\begin{array}{cc}
\frac{a_{4}+a_{1}}{2}, & \text { si } w_{\widetilde{A}}=0
\end{array}\right. \\
\frac{w_{\widetilde{A}}\left(\frac{a_{3}-a_{2}}{\left.a_{4}-a_{1}+2\right)}\right.}{6}, & \text { si } a_{4}-a_{1} \neq 0 \\
\frac{w_{\widetilde{A}}}{2}, & \text { si } a_{4}-a_{1}=0
\end{array}\right.
\end{gathered}
$$

$\mathrm{y}$

$$
\begin{gathered}
B\left(S_{\widetilde{A}}, S_{\widetilde{B}}\right)=\left\{\begin{array}{l}
1, \quad \text { si } S_{\widetilde{A}}+S_{\widetilde{B}}>0 \\
0, \quad \text { en otro caso }
\end{array}\right. \\
S_{\widetilde{A}}=a_{4}-a_{1} \\
S_{\widetilde{B}}=b_{4}-b_{1}
\end{gathered}
$$

El factor $\left[1-X_{\widetilde{A}}-X_{\widetilde{B}}\right]^{B\left(S_{\widetilde{A}}, S_{\widetilde{B}}\right)}$ se usa para distinguir los pares $\widetilde{A}=\left(a, a, a, a ; w_{\widetilde{A}}\right) \mathrm{y}$ $\widetilde{B}=\left(b, b, b, b ; w_{\widetilde{B}}\right)$ del resto de pares de números borrosos generalizados. 
Wei y Chen [76] propusieron una nueva medida en 2009 utilizando en esta ocasión el concepto de perímetro de los números borrosos trapezoidales generalizados:

$$
S(\widetilde{A}, \widetilde{B})=\left[1-\frac{\sum_{i=1}^{4}\left|a_{i}-b_{i}\right|}{4}\right] \times\left[\frac{\min \{P(\widetilde{A}), P(\widetilde{B})\}+\min \left\{w_{\widetilde{A}}, w_{\widetilde{B}}\right\}}{\max \{P(\widetilde{A}), P(\widetilde{B})\}+\max \left\{w_{\widetilde{A}}, w_{\widetilde{B}}\right\}}\right],
$$

donde $P(\widetilde{A})=\sqrt{\left(a_{1}-a_{2}\right)^{2}+w_{\widetilde{A}}^{2}}+\sqrt{\left(a_{3}-a_{4}\right)^{2}+w_{\widetilde{A}}^{2}}+\left(a_{3}-a_{2}\right)+\left(a_{4}-a_{1}\right)$ y de manera análoga para $P(\widetilde{B})$.

En 2010 Xu et al. [78] proponen una nueva medida basada, como la de Chen de 2003, en el concepto de centro de gravedad pero intentan utilizarla también en números borrosos trapezoidales con altura nula, es decir, con $w_{\widetilde{A}}=0$.

En la medida de $\mathrm{Xu}$ et al. se consideran dos pesos $w, 1-w \in(0,1)$ para dar mayor o menor importancia a los conceptos utilizados:

$$
S_{w}(\widetilde{A}, \widetilde{B})=1-w \frac{\sum\left|a_{i}-b_{i}\right|}{4}-(1-w) \frac{\sqrt{\left(X_{\widetilde{A}}-X_{\widetilde{B}}\right)^{2}+\left(Y_{\widetilde{A}}-Y_{\widetilde{B}}\right)^{2}}}{\sqrt{1.25}} .
$$

Esta medida aportaría una nueva propiedad:

$$
S(\widetilde{A}, \widetilde{B})=0(y \widetilde{A}<\widetilde{B}) \Longleftrightarrow \widetilde{A}=(0,0,0,0 ; 0) \text { y } \widetilde{B}=(1,1,1,1 ; 1) .
$$

Sin embargo, los números borrosos trapezoidales de altura nula no tienen sentido según la propia definición de función de pertenencia, ya que todos los números reales tendrían pertenencia nula al número borroso de altura nula.

Además, Xu et al. sacrifican la tercera propiedad para conseguir ésta. Por ejemplo, para $(a, a, a, a ; 1)$ y $(b, b, b, b ; 1)$ se tiene

$$
S(\widetilde{A}, \widetilde{B})=1-0.5|a-b|-0.5 \frac{|a-b|}{\sqrt{1.25}} \neq 1-|a-b|,
$$

contra la tercera propiedad.

Otro error de la medida de Xu se observa, por ejemplo, si consideramos los números trapezoidales borrosos dados (véase la Figura 4.1.1) por $\widetilde{A}=(0,0.1,0.3,0.4 ; 1), \widetilde{B}=(0.25$, $0.4,0.6,0.75 ; 1)$ у $\widetilde{C}=(0.75,0.75,0.825,0.85 ; 1)$. El grado de similitud con $w=0.5$ de $\widetilde{C}$ y $\widetilde{A}$ con respecto a $\widetilde{B}$ es 0.715629 en ambos casos, y por tanto, los números de los extremos son igual de parecidos al número central. Sin embargo, parece claro que tanto por la forma, como por el tamaño, y lo que puede ser más importante, por compartir área, $\widetilde{B}$ debe ser más parecido a $\widetilde{A}$ que a $\widetilde{C}$. 


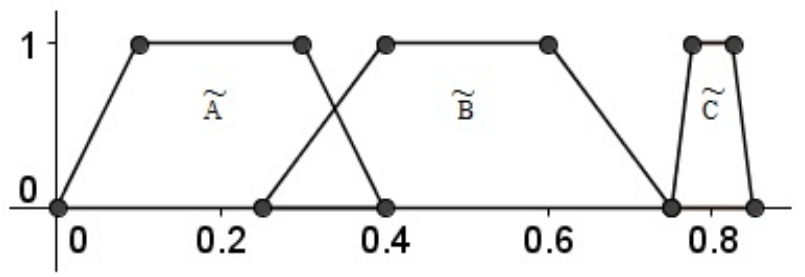

Figura 4.1.1: Un fallo en la función de similitud de Xu et al [78]

Además de las medidas de Wei y Chen, y de Xu, muchos otros autores han definido el grado de similitud entre dos números borrosos trapezoidales. Por su actualidad destacaremos las siguientes medidas:

- Función de Sridevi y Nadarajan de 2009 [55] (sacrifica la tercera propiedad). Está basada en la diferencia borrosa de distancia entre dos números borrosos:

$$
S(\widetilde{A}, \widetilde{B})=\left[1-\frac{\sum_{i=1}^{4} \mu_{d}(x)}{4}\right] \times\left[1-X_{\widetilde{A}}-X_{\widetilde{B}}\right]^{B\left(S_{\widetilde{A}}, S_{\widetilde{B}}\right)} \times\left[\frac{\min \left\{Y_{\widetilde{A}}, Y_{\widetilde{B}}\right\}}{\max \left\{Y_{\widetilde{A}}, Y_{\widetilde{B}}\right\}}\right],
$$

$\operatorname{con} \mu_{d}(x)=\left\{\begin{array}{cc}1-\frac{x}{d}, & \text { si } 0 \leq x \leq d \\ 0, & \text { en otro caso }\end{array}, d \in(0,1], x=\left|a_{i}-b_{i}\right| \mathrm{y}\left(X_{\widetilde{A}}, Y_{\widetilde{A}}\right) \mathrm{y}\left(X_{\widetilde{B}}, Y_{\widetilde{B}}\right)\right.$ los centroides de los números comparados.

La elección del parámetro $d$ representa el grado de precisión requerido para medir la similitud entre números borrosos.

- Función de Gomathi y Sivaraman de 2012 [23]. Esta función modifica la de Wei y Chen utilizando la media geométrica de la diferencia de los vértices de los números comparados, en lugar de la media aritmética. Otra modificación con respecto a la medida de Wei y Chen es que en lugar de utilizar el perímetro del número trapezoidal usan una función más sencilla de los vértices y la altura de los números trapezoidales borrosos generalizados. Su intención es reducir el tiempo computacional de la medida de Wei and Chen, obteniendo valores similares [76]

$$
S(\widetilde{A}, \widetilde{B})=\left[\sqrt[4]{\prod_{i=1}^{4}\left(1-\left|a_{i}-b_{i}\right|\right)}\right] \times\left[\frac{\min \{Q(\widetilde{A}), Q(\widetilde{B})\}+\min \left\{w_{\widetilde{A}}, w_{\widetilde{B}}\right\}}{\max \{Q(\widetilde{A}), Q(\widetilde{B})\}+\max \left\{w_{\widetilde{A}}, w_{\widetilde{B}}\right\}}\right],
$$

donde

$$
Q(\widetilde{A})=\sqrt{\left(a_{2}-a_{1}\right)^{2}+\left(a_{3}-a_{2}\right)^{2}+\left(a_{4}-a_{3}\right)^{2}+w_{\widetilde{A}}^{2}},
$$


y análogamente para $Q(\widetilde{B})$

- Función de Hejazi et al. de 2011 [20]:

$$
\begin{aligned}
S(\widetilde{A}, \widetilde{B})= & {\left[1-\frac{\sum_{i=1}^{4}\left|a_{i}-b_{i}\right|}{4}\right] \times\left[\frac{\min \{P(\widetilde{A}), P(\widetilde{B})\}}{\max \{P(\widetilde{A}), P(\widetilde{B})\}}\right] \times } \\
& {\left[\frac{\min A\{(\widetilde{A}), A(\widetilde{B})\}+\min \left\{w_{\widetilde{A}}, w_{\widetilde{B}}\right\}}{\max \{A(\widetilde{A}), A(\widetilde{B})\}+\max \left\{w_{\widetilde{A}}, w_{\widetilde{B}}\right\}}\right], }
\end{aligned}
$$

donde $P(\widetilde{A})=\sqrt{\left(a_{1}-a_{2}\right)^{2}+w_{\widetilde{A}}^{2}}+\sqrt{\left(a_{3}-a_{4}\right)^{2}+w_{\widetilde{A}}^{2}}+\left(a_{3}-a_{2}\right)+\left(a_{4}-a_{1}\right)$ es el perímetro de $\widetilde{A}$, y análogamente con $P(\widetilde{B})$, y $A(\widetilde{A})=\frac{\left[\left(a_{3}-a_{2}\right)+\left(a_{4}-a_{1}\right)\right] w_{\widetilde{A}}}{2}$ es el área de $\widetilde{A}$ y análogamente con $A(\widetilde{B})$.

- Función de Zhu y Xu de 2012 [85]:

Si $\max \{P(\widetilde{A}), P(\widetilde{B})\} \neq 0$, entonces

$$
\begin{aligned}
S(\widetilde{A}, \widetilde{B})= & \sqrt{\left[1-\frac{\sum_{i=1}^{4}\left|a_{i}-b_{i}\right|}{4}\right] \times\left[1-\sqrt{\left(X_{\widetilde{A}}-X_{\widetilde{B}}\right)^{2}+\left(Y_{\widetilde{A}}-Y_{\widetilde{B}}\right)^{2}}\right] \times} \\
& \times\left[\frac{\min \{P(\widetilde{A}), P(\widetilde{B})\}}{\max \{P(\widetilde{A}), P(\widetilde{B})\}}\right] \times e^{\left[\frac{\min \{A(\widetilde{A}), A(\widetilde{B})\}}{\max \{A(\widetilde{A}), A(\tilde{B})\}}\right]} .
\end{aligned}
$$

Si $\max \{P(\widetilde{A}), P(\widetilde{B})\}=0$, entonces

$$
S(\widetilde{A}, \widetilde{B})=\sqrt{\left[1-\frac{\sum_{i=1}^{4}\left|a_{i}-b_{i}\right|}{4}\right] \times\left[1-\sqrt{\left(X_{\widetilde{A}}-X_{\widetilde{B}}\right)^{2}+\left(Y_{\widetilde{A}}-Y_{\widetilde{B}}\right)^{2}}\right] .}
$$

Esta función tiene un grave problema de definición, ya que no es capaz de medir la similitud entre pares de números borrosos cuyos centroides estén a una distancia superior a 1. Por ejemplo la similitud de los números $(0.01,0.01,0.01,0.01 ; 0.5)$ y $(1,1,1,1 ; 1)$ no está definida con la expresión de Zhu y Xu, ya que resulta

$$
\sqrt{1-0.99} \sqrt{1-\sqrt{0.99^{2}+0.25^{2}}}=\sqrt{0.01} \sqrt{-0.021}
$$


Este error se podría subsanar dividiendo como hace Xu [78] en 2010 por $\sqrt{1.25}$, que es la máxima distancia entre los centroides de los números trapezoidales generalizados con soporte en $[0,1]$, pero en tal caso, al igual que le ocurre a la función de Xu et al. sacrificaríamos la propiedad 3 .

- Función de Vicente et al. de 2013 [62, 68, 71]:

Definimos el grado de similitud entre los números borrosos trapezoidales generali$\operatorname{zados} \widetilde{A}=\left(a_{1}, a_{2}, a_{3}, a_{4} ; w_{\widetilde{A}}\right)$ y $\widetilde{B}=\left(b_{1}, b_{2}, b_{3}, b_{4} ; w_{\widetilde{B}}\right)$ como:

- Si $\int_{0}^{1} \mu_{\widetilde{A} \cup \widetilde{B}}(x) d x \neq 0$, entonces

$$
\begin{gathered}
S(\widetilde{A}, \widetilde{B})=\left(1-\left|w_{\widetilde{A}}-w_{\widetilde{B}}\right|\right)\left[1-(1-\alpha-\beta)\left(1-\frac{\int_{0}^{1} \mu_{\widetilde{A} \cap \widetilde{B}}(x) d x}{\int_{0}^{1} \mu_{\widetilde{A} \cup \widetilde{B}}(x) d x}\right)-\right. \\
\left.-\alpha \frac{\sum\left|a_{i}-b_{i}\right|}{4}-\beta \frac{d\left[\left(X_{\widetilde{A}}, Y_{\widetilde{A}}\right),\left(X_{\widetilde{B}}, Y_{\widetilde{B}}\right)\right]}{M}\right] .
\end{gathered}
$$

- Si $\int_{0}^{1} \mu_{\widetilde{A} \cup \widetilde{B}}(x) d x=0$, entonces

$$
\begin{aligned}
S(\widetilde{A}, \widetilde{B})= & \left(1-\left|w_{\widetilde{A}}-w_{\widetilde{B}}\right|\right)\left[1-\left(\frac{1-\alpha-\beta}{2}+\alpha\right) \frac{\sum\left|a_{i}-b_{i}\right|}{4}-\right. \\
& \left.-\left(\frac{1-\alpha-\beta}{2}+\beta\right) \frac{d\left[\left(X_{\widetilde{A}}, Y_{\widetilde{A}}\right),\left(X_{\widetilde{B}}, Y_{\widetilde{B}}\right)\right]}{M}\right] .
\end{aligned}
$$

La función de Vicente et al. fue definida para incorporar el área común entre los números borrosos comparados en relación al área total y pueden verse numerosas ventajas con respecto a otras funciones de similitud en [62].

\subsection{Similitud de consenso}

Para comparar las diversas funciones de similitud suele recurrirse a conjuntos arbitrarios de números borrosos en los que pueden verse las ventajas de la función propuesta pero no sus inconvenientes. Por ejemplo, un conjunto clásico son los quince pares de Chen que pueden verse en la Figura 4.2.1. La mayoría de los autores suelen ampliar este conjunto con varios pares más, como Sridevi y Nadarajan, que utilizan 26 pares, pero otros autores utilizan conjuntos de pares verdaderamente pequeños, como Gomathi y Sivaraman que utilizan cinco pares de números para comparar su función de similitud con el resto de funciones. 


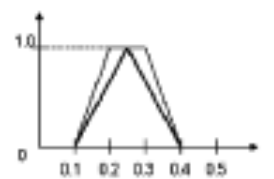

$\lambda=(0.1,0.2,0.3,0.4,10)$

$\delta=(01,0.25,0.25,04,1.9$

Sat 1

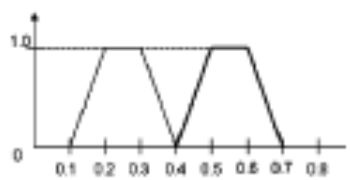

$\tilde{\lambda}=(0.1,0.2,0.3,0.4,1.0)$

$\overline{\mathbf{b}}-(0,4,0.5,0.5,0.7,10)$

Set 4

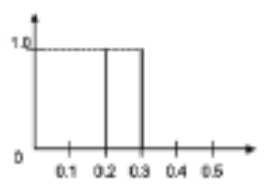

$\overline{\boldsymbol{A}}=(0.2,0.2,0.2,0.2,1,0)$

$\tilde{g}=(0.3,0.3,0.3,0.3,1.0)$

Set 7

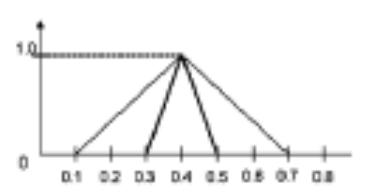

$x-(0.1,0.4,0.4,0.7,1.6)$

$\tilde{\boldsymbol{B}}=\{0.2,0.4,0.4,0.5,2.0)$

Set 10

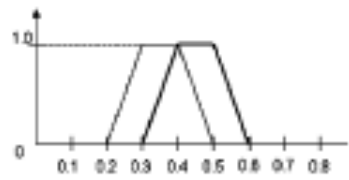

$\tilde{\boldsymbol{i}}=(0.2,03,0.4,0.5,1.0)$

$\bar{b}=(0.3,0.4,0.5,0.6,1.0)$

Set 13

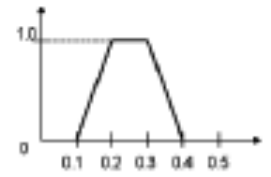

$\bar{A}=(0.1,0.2,0.3,0.4 ; 10)$

$\bar{z}=(0.1,0.2,0.3,0.4 ; 10)$

Set 2

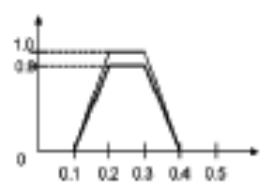

$\bar{A}=(01,0.2,0.3,04,1.0)$

$\bar{B}=(0.1,0.2,0.1,0.4,0.89$

Set 5

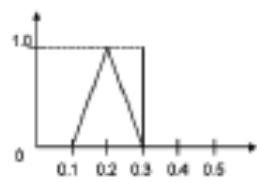

$\vec{A}=(\alpha, 1,0.2,0.2,0.2,0)$

$B=(0.3,0.3,0.3,0.3 .1 .5)$

Set $B$

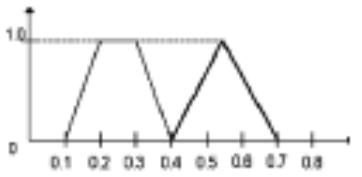

$\bar{\lambda}=(0.1,0.2,0.3,0.4 ; 1.0)$

$\tilde{f}=(0.4,0.55,0.55,07,1.0)$

$\operatorname{Set} 3$

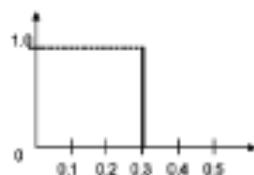

$\tilde{\lambda}=(0.3,0.3,03,0.3,1.0)$

$\overline{\mathbf{B}}=(0.3,0.3,0.3,0.3,1.0)$

Set 6

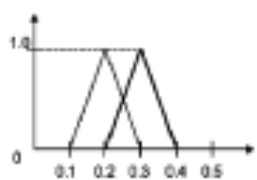

$\bar{A}=(0.2,0.2,0.2,0.2,10)$

$\overline{8}=(0.2,0.3,0.3,0.4,1.0)$

Set 9

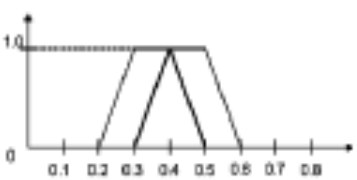

$X-10.2,0.3,0.5,0.5,1.0 \%$

$\tilde{s}=(0.3,0.4,0.4,0.5,10)$

Set 11

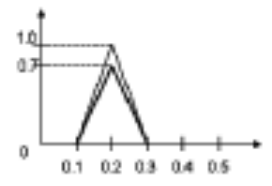

$\tilde{u}-(0.1,0.2,0.2,0.3 .0)$

b-(0.1, 0.2, 0.2, 0.3, 0.7)

Set 14

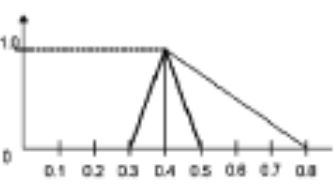

$X-(04,04,04,0 \& 1.0)$

$\tilde{b}=(0,3,04,04,0.2,19)$

Set 12

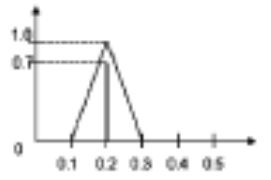

$\tilde{\boldsymbol{A}}-(0.1,0.2,0.3,0.4 .0 \%)$

fi $-(0.2,0.2,0.2,0.2,0.7)$

Set 15

Figura 4.2.1: Pares de comparación de Chen y Chen [11]

Además, aunque el hecho de utilizar números trapezoidales con altura nula pudiera parecer un error aislado en la literatura, sin embargo no son pocos los autores que los han utilizado contradiciendo incluso las definiciones de números trapezoidales que exponen en sus propios artículos, como es el caso de Xu et al. o Gomathi y Sivaraman.

En Vicente et al. [62] se comparan funciones dos a dos representándolas gráficamente sobre un par de ejes coordenados. Si en la abscisa consideramos una función y en la ordenada consideramos otra, entonces estas funciones serán más parecidas cuanto más cercanos estén los puntos a la biseción del primer cuadrante, y serán completamente 
opuestas si los puntos obtenidos se situan próximos a la recta $y=1-x$.

Por tanto, el coeficiente de correlación de Pearson nos informa sobre el parecido que existe entre dos funciones de similitud sobre un conjunto de pares de números borrosos trapezoidales a comparar.

En la Tabla 4.2.1 podemos ver la matriz de coeficientes de correlación de Pearson de las funciones en el orden: (1) Wei y Chen (2009) [76], (2) Xu et al. (2010) [78], (3) Gomathi (2010) [23], (4) Chen y Chen (2003) [11], (5) Wen et al. (2011) [77], (6) Zhu y Xu (2012) [85], (7) Vicente et al. (2013) [62], (8) Sridevi y Nadarajan (2009) [55].

Tabla 4.2.1: Coeficientes de correlación de Pearson para comparar de las funciones consideradas sobre los pares de Chen y Chen de 2003 [11]

\begin{tabular}{ccccccccc}
\hline Función & 1 & 2 & 3 & 4 & 5 & 6 & 7 & 8 \\
\hline \hline 1 & 1.00000 & 0.52536 & 0.86011 & 0.62610 & 0.93797 & 0.75587 & 0.88030 & 0.98588 \\
\hline 2 & 0.52536 & 1.00000 & 0.25831 & 0.89314 & 0.28418 & 0.23917 & 0.49060 & 0.44893 \\
\hline 3 & 0.86011 & 0.25831 & 1.00000 & 0.41214 & 0.93644 & 0.51051 & 0.94355 & 0.86389 \\
\hline 4 & 0.62610 & 0.89314 & 0.41214 & 1.00000 & 0.48144 & 0.48124 & 0.61249 & 0.52661 \\
\hline 5 & 0.93797 & 0.28418 & 0.93644 & 0.48144 & 1.00000 & 0.75057 & 0.90138 & 0.94313 \\
\hline 6 & 0.75587 & 0.23917 & 0.51051 & 0.48124 & 0.75057 & 1.00000 & 0.46482 & 0.74866 \\
\hline 7 & 0.88030 & 0.49060 & 0.94355 & 0.61249 & 0.90138 & 0.46482 & 1.00000 & 0.85160 \\
\hline 8 & 0.98588 & 0.44893 & 0.86389 & 0.52661 & 0.94313 & 0.74866 & 0.85160 & 1.00000 \\
\hline
\end{tabular}

Por ejemplo, observamos un gran parecido entre las funciones de Wei y Chen de 2009, la de Sridevi y Nadarajan, y la de Wen et al., y una gran discordancia entre la función de Gomathi y Sivaraman y la de Xu et al.

En la Figura 4.2.2 podemos ver el comportamiento de todas las funciones de similitud indicadas sobre los pares del conjunto de comparación de Chen. En ella observamos una gran variabilidad en la identificación de similitud de los pares comparados. Por ejemplo, vemos que la función de $\mathrm{Xu}$ et al. generalmente otorga una alta similitud, por encima del resto de funciones. La de Zhu y Xu es bastante exigente y generalmente puntúa por debajo del resto, aunque hay pares a los cuales otorga una similitud superior a la otorgada por el resto. Finalmente, existen funciones muy inestables, en el sentido de que unas veces otorgan similitudes por debajo del resto de funciones y otras veces por encima. 


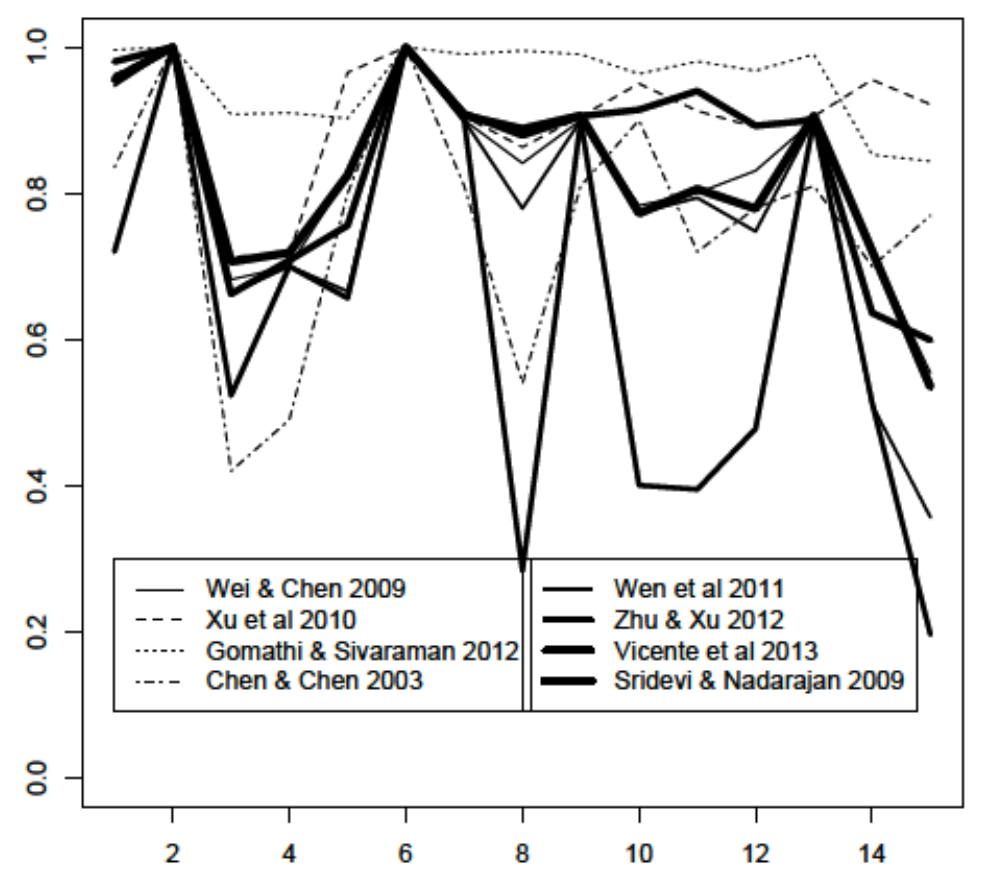

Figura 4.2.2: Funciones de similitud consideradas sobre el conjunto de pares de Chen y Chen [11].

Ante tal variabilidad e inestabilidad tiene sentido establecer un consenso y tratar de obtener una función estable que tenga en cuenta de manera ponderada todos los puntos de vista expresados por las funciones de la literatura. Este consenso puede estar dado por varios criterios como pueden ser la media o el coeficiente de correlación de Pearson. Es decir, una función de consenso puede estar definida, entre otros criterios, por la media entre varias funciones de similitud sobre un conjunto de pares de números borrosos trapezoidales, o por una función que maximice el coeficiente de correlación de Pearson respecto a cada función de similitud considerada. Nuestro objetivo es encontrar la expresión analítica de una función de similitud que satisfaga estos criterios.

\subsubsection{Función de similitud de consenso}

Como podemos apreciar en la literatura, los parámetros más utilizados en las funciones de similitud son las distancias geométrica y aritmética, la distancia entre los centros de gravedad, y la diferencia entre las alturas, los perímetros y las áreas. Consideraremos las siguientes familias de funciones de similitud.

\section{Proposición.}

Las siguientes funciones son de similitud. 
- Familia 1.

$S_{1}(\widetilde{A}, \widetilde{B})=1-\left(w_{1} \frac{\sum\left|a_{i}-b_{i}\right|}{4}+w_{2} d\left(\left(X_{\widetilde{A}}, Y_{\widetilde{A}}\right),\left(X_{\widetilde{B}}, Y_{\widetilde{B}}\right)\right)+w_{3} \sqrt[4]{\prod_{i=1}^{4}\left|a_{i}-b_{i}\right|}\right)$

$\operatorname{con} w_{1}, w_{2}, w_{3} \in[0,1]$ y $w_{1}+w_{2}+w_{3}=1$.

- Familia 2.

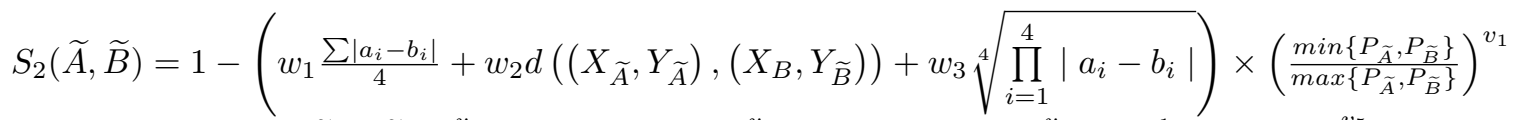

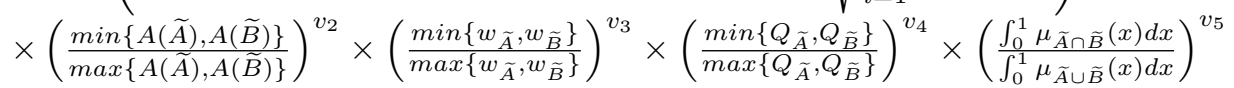

con $w_{1}, w_{2}, w_{3}, \in[0,1]: \sum w_{j i}=1, \mathrm{y} v_{1}, v_{2}, v_{3}, v_{4}, v_{5} \in\{0,1\}$

Esta familia generaliza a la Familia 1 sin más que tomar $v_{i}=0 \forall i$.

- Familia 3.

$$
\begin{aligned}
& S_{3}(\widetilde{A}, \widetilde{B})=1-\left(w_{1} \frac{\sum\left|a_{i}-b_{i}\right|}{4}+w_{2} d\left(\left(X_{\widetilde{A}}, Y_{\widetilde{A}}\right),\left(X_{\widetilde{B}}, Y_{\widetilde{B}}\right)\right)+w_{3} \sqrt[4]{\prod_{i=1}^{4}\left|a_{i}-b_{i}\right|}\right) \times \\
& \quad \times \prod_{i=1}^{n}\left(\frac{v_{1 i} \min \left\{P_{\widetilde{A}}, P_{\widetilde{B}}\right\}+v_{2 i} \min \{A(\widetilde{A}), A(\widetilde{B})\}+v_{3 i} \min \left\{w_{\widetilde{A}}, w_{\widetilde{B}}\right\}+v_{4 i} \min \left\{Q_{\widetilde{A}}, Q_{\widetilde{B}}\right\}+v_{5 i} \int_{0}^{1} \mu_{\widetilde{A} \cap \widetilde{B}}(x) d x}{v_{1 i} \max \left\{P_{\widetilde{A}}, P_{\widetilde{B}}\right\}+v_{2 i} \max \{A(\widetilde{A}), A(\widetilde{B})\}+v_{3 i} \max \left\{w_{\widetilde{A}}, w_{\widetilde{B}}\right\}+v_{4 i} \max \left\{Q_{\widetilde{A}}, Q_{\widetilde{B}}\right\}+v_{5 i} \int_{0}^{1} \mu_{\widetilde{A} \cup \widetilde{B}}(x) d x}\right)
\end{aligned}
$$

con $w_{1}, w_{2}, w_{3}, v_{1 i}, v_{2 i}, v_{3 i}, v_{4 i}, v_{5 i} \in[0,1] \mathrm{y} \sum w_{k}=1, \sum v_{j i}=1$.

Esta familia generaliza a la anterior sin más que tomar $n=5 \mathrm{y}\left(v_{i}=1, v_{j}=0 \forall i \neq j\right)$ $\forall i=1, \ldots, 5$

- Familia 4.

$$
\begin{aligned}
& S_{4}(\widetilde{A}, \widetilde{B})=\left[1-\left(w_{1} \frac{\sum\left|a_{i}-b_{i}\right|}{4}+w_{2} d\left(\left(X_{\widetilde{A}}, Y_{\widetilde{A}}\right),\left(X_{\widetilde{B}}, Y_{\widetilde{B}}\right)\right)+w_{3} \sqrt[4]{\prod_{i=1}^{4}\left|a_{i}-b_{i}\right|}\right)\right] \times \\
& \quad \times \prod_{i=1}^{n}\left(\frac{v_{1 i} \min \left\{P_{\widetilde{A}}, P_{\widetilde{B}}\right\}+v_{2 i} \min \{A(\widetilde{A}), A(\widetilde{B})\}+v_{3 i} \min \left\{w_{\widetilde{A}}, w_{\widetilde{B}}\right\}+v_{4 i} \min \left\{Q_{\widetilde{A}}, Q_{\widetilde{B}}\right\}+v_{5 i} \int_{0}^{1} \mu_{\widetilde{A} \cap \widetilde{B}}(x) d x}{v_{1 i} \max \left\{P_{\widetilde{A}}, P_{\widetilde{B}}\right\}+v_{2 i} \max \{A(\widetilde{A}), A(\widetilde{B})\}+v_{3 i} \max \left\{w_{\widetilde{A}}, w_{\widetilde{B}}\right\}+v_{4 i} \max \left\{Q_{\widetilde{A}}, Q_{\widetilde{B}}\right\}+v_{5 i} \int_{0}^{1} \mu_{\widetilde{A} \cup \widetilde{B}}(x) d x}\right)
\end{aligned}
$$

con $w_{1}, w_{2}, w_{3}, v_{1 i}, v_{2 i}, v_{3 i}, v_{4 i}, v_{5 i} \in[0,1] \mathrm{y} \sum w_{k}=1, \sum v_{j i}=1$.

\section{Demostración.}

Para la Familia 1, puesto que $\frac{\sum\left|a_{i}-b_{i}\right|}{4}, d\left(\left(X_{\widetilde{A}}, Y_{\widetilde{A}}\right),\left(X_{B}, Y_{\widetilde{B}}\right)\right), \sqrt[4]{\prod_{i=1}^{4}\left|a_{i}-b_{i}\right|} \in[0,1]$, y dado que $w_{1}, w_{2}, w_{3} \in[0,1]$, con $w_{1}+w_{2}+w_{3}=1$, es trivial que $0 \leq S_{1}(\widetilde{A}, \widetilde{B}) \leq 1$ 
Además, $S_{1}(\widetilde{A}, \widetilde{B})=S_{1}(\widetilde{B}, \widetilde{A})$ porque todos los elementos considerados son simétricos, $\mathrm{y}$

$$
S_{1}(\widetilde{A}, \widetilde{B})=1 \Leftrightarrow \frac{\sum\left|a_{i}-b_{i}\right|}{4}=d\left(\left(X_{\widetilde{A}}, Y_{\widetilde{A}}\right),\left(X_{\widetilde{B}}, Y_{\widetilde{B}}\right)\right)=\sqrt[4]{\prod_{i=1}^{4}\left|a_{i}-b_{i}\right|}=0 \Leftrightarrow \widetilde{A}=\widetilde{B} .
$$

Finalmente,

$S_{1}(\widetilde{a}, \widetilde{b})=1-\left(w_{1}|a-b|+w_{2}|a-b|+w_{3}|a-b|\right)=1-\left(w_{1}+w_{2}+w_{3}\right)|a-b|=1-|a-b|$

La Familia 2 está bien definida porque añadimos a la Familia 1 un producto de factores que están en [0,1]. Además, estos factores son simétricos, lo que garantiza la propiedad 1. La propiedad 2 se verifica por la misma razón que se verifica para la Familia 1, ya que $S_{2}(\widetilde{A}, \widetilde{B})=1 \Leftrightarrow\left(w_{1} \frac{\sum\left|a_{i}-b_{i}\right|}{4}+w_{2} d\left(\left(X_{\widetilde{A}}, Y_{\widetilde{A}}\right),\left(X_{\widetilde{B}}, Y_{\widetilde{B}}\right)\right)+w_{3} \sqrt[4]{\prod_{i=1}^{4}\left|a_{i}-b_{i}\right|}\right)=0$. La tercera propiedad se verifica porque para números reales el producto añadido a la primera familia resulta 1 .

En cuanto a la Familia 3, la función está bien definida porque cada uno de los factores considerados está bien definido y es menor o igual que 1.

El primer factor es trivialmente menor o igual que 1 porque

$$
\frac{\sum\left|a_{i}-b_{i}\right|}{4}, d\left(\left(X_{\widetilde{A}}, Y_{\widetilde{A}}\right),\left(X_{\widetilde{B}}, Y_{\widetilde{B}}\right)\right), \sqrt[4]{\prod_{i=1}^{4}\left|a_{i}-b_{i}\right|} \in[0,1]
$$

y $w_{1}, w_{2}, w_{3} \in[0,1]$ con $\sum w_{i}=1$.

El segundo factor está bien definido porque $v_{1 i} \max \left\{P_{\widetilde{A}}, P_{\widetilde{B}}\right\}+v_{2 i} \max \{A(\widetilde{A}), A(\widetilde{B})\}+$ $v_{3 i} \max \left\{w_{\widetilde{A}}, w_{\widetilde{B}}\right\}+v_{4 i} \max \left\{Q_{\widetilde{A}}, Q_{\widetilde{B}}\right\}+v_{5 i} \int_{0}^{1} \mu_{\widetilde{A} \cup \widetilde{B}}(x) \geq v_{1 i} \max \left\{P_{\widetilde{A}}, P_{\widetilde{B}}\right\}+v_{3 i} \max \left\{w_{\widetilde{A}}, w_{\widetilde{B}}\right\}$ es positivo y es trivialmente menor o igual que 1 porque el denominador siempre es mayor o igual que el numerador. Nótese además que $\frac{v_{1 i} \min \left\{P_{\widetilde{A}}, P_{\widetilde{B}}\right\}+v_{2 i} \min \{A(\widetilde{A}), A(\widetilde{B})\}+v_{3 i} \min \left\{w_{\widetilde{A}}, w_{\widetilde{B}}\right\}+v_{4 i} \min \left\{Q_{\widetilde{A}}, Q_{\widetilde{B}}\right\}+v_{5 i} \int_{0}^{1} \mu_{\widetilde{A} \cap \widetilde{B}}(x) d x}{v_{1 i} \max \left\{P_{\widetilde{A}}, P_{\widetilde{B}}\right\}+v_{2 i} \max \{A(\widetilde{A}), A(\widetilde{B})\}+v_{3 i} \max \left\{w_{\widetilde{A}}, w_{\widetilde{B}}\right\}+v_{4 i} \max \left\{Q_{\widetilde{A}}, Q_{\widetilde{B}}\right\}+v_{5 i} \int_{0}^{1} \mu_{\widetilde{A} \cup \widetilde{B}}(x) d x}>0$ Ahora veamos que se cumplen las tres propiedades:

1. $S_{3}(\widetilde{A}, \widetilde{B})=S_{3}(\widetilde{B}, \widetilde{A})$. Trivial porque todos los elementos considerados son simétri$\cos$.

2. $S_{3}(\widetilde{A}, \widetilde{B})=1 \Leftrightarrow \widetilde{A}=\widetilde{B}$. 
Supongamos que $S_{3}(\widetilde{A}, \widetilde{B})=1$, entonces

$$
w_{1} \frac{\sum\left|a_{i}-b_{i}\right|}{4}+w_{2} d\left(\left(X_{\widetilde{A}}, Y_{\widetilde{A}}\right),\left(X_{\widetilde{B}}, Y_{\widetilde{B}}\right)\right)+w_{3} \sqrt[4]{\prod_{i=1}^{4}\left|a_{i}-b_{i}\right|}=0
$$

ya que, como hemos indicado anteriormente, el segundo factor nunca se anula.

Por tanto, $\frac{\sum\left|a_{i}-b_{i}\right|}{4}=0, d\left(\left(X_{\widetilde{A}}, Y_{\widetilde{A}}\right),\left(X_{B}, Y_{\widetilde{B}}\right)\right)=0, \sqrt[4]{\prod_{i=1}^{4}\left|a_{i}-b_{i}\right|}=0$. Todas estas igualdades confirman que $a_{i}=b_{i} \forall i=1, . ., 4$ y particularmente la segunda igualdad confirma además que $w_{\widetilde{A}}=w_{\widetilde{B}}$. Luego, en efecto $\widetilde{A}=\widetilde{B}$.

La implicación inversa es trivial, dado que la función está bien definida.

3. $S_{3}(\widetilde{A}, \widetilde{B})=1-|a-b|$.

Puesto que el segundo factor es igual a 1 se tiene que

$S_{3}(\widetilde{a}, \widetilde{b})=1-\left(w_{1}|a-b|+w_{2}|a-b|+w_{3}|a-b|\right)=1-\left(w_{1}+w_{2}+w_{3}\right)|a-b|=$ $1-|a-b|$.

Finalmente, que la función de la Familia 4 está bien definida se demuestra fácilmente de modo análogo a las funciones de las familias anteriores.

La función es simétrica porque son simétricos todos los elementos considerados. Además,

$$
\begin{aligned}
& S_{4}(\widetilde{A}, \widetilde{B})=1 \Leftrightarrow w_{1} \frac{\sum\left|a_{i}-b_{i}\right|}{4}+w_{2} d\left(\left(X_{\widetilde{A}}, Y_{\widetilde{A}}\right),\left(X_{\widetilde{B}}, Y_{\widetilde{B}}\right)\right)+w_{3} \sqrt[4]{\prod_{i=1}^{4}\left|a_{i}-b_{i}\right|}=0 \\
& \mathrm{y} \frac{v_{1 i} \min \left\{P_{\widetilde{A}}, P_{\widetilde{B}}\right\}+v_{2 i} \min \{A(\widetilde{A}), A(\widetilde{B})\}+v_{3 i} \min \left\{w_{\widetilde{A}}, w_{\widetilde{B}}\right\}+v_{4 i} \min \left\{Q_{\widetilde{A}}, Q_{\widetilde{B}}\right\}+v_{5 i} \int_{0}^{1} \mu_{\widetilde{A}} \mu_{\widetilde{B}}(x) d x}{v_{1 i} \max \left\{P_{\widetilde{A}}, P_{\widetilde{B}}\right\}+v_{2 i} \max \{A(\widetilde{A}), A(\widetilde{B})\}+v_{3 i} \max \left\{w_{\widetilde{A}}, w_{\widetilde{B}}\right\}+v_{4 i} \max \left\{Q_{\widetilde{A}}, Q_{\widetilde{B}}\right\}+v_{5 i} \int_{0}^{1} \mu_{\widetilde{A} \cup \widetilde{B}}(x) d x}=1 \forall i .
\end{aligned}
$$

y la primera igualdad se sigue si y sólo si $\widetilde{A}=\widetilde{B}$. Finalmente, como en los casos anteriores: $S_{4}(\widetilde{a}, \widetilde{b})=1-\left(w_{1}|a-b|+w_{2}|a-b|+w_{3}|a-b|\right)=1-\left(w_{1}+w_{2}+w_{3}\right)|a-b|=1-|a-b|$

Hemos generado aleatoriamente cien combinaciones de los pesos para las familias 1, $2,3$ ( $\operatorname{con} n=1)$ y 4 . Consideremos en primer lugar como consenso el criterio de la media, es decir, buscamos la expresión analítica de una función de similitud que se aproxime a la media de las funciones consideradas sobre los quince pares de Chen.

Como puede verse en la Figura 4.2.2, las funciones de similitud de Ghomathi y Sivaraman y de Zhu y Xu constituyen dos datos atípicos en la muestra de funciones dadas. La primera da valores excesivamente altos para todos los pares de números borrosos. La segunda da valores inusualmente bajos. Estas dos funciones no serán consideradas en el resto del paper por generar datos atípicos. 
Las curvas que representan las funciones de similitud resultantes pueden verse en la Figura 4.2.3, en la que se ha señalado con trazo grueso la media de todas las funciones de similitud consideradas sobre los quince pares de números borrosos trapezoidales de Chen. Como puede verse, las funciones de similitud de la Familia 1 tienen dificultades para consensuar la similitud de los últimos cinco pares (salvo el par 13), mientras que ajusta relativamente bien la mayoría de los primeros nueve pares (salvo el par 5).

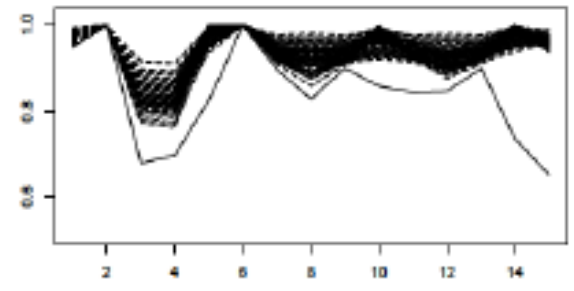

(a) Famila 1

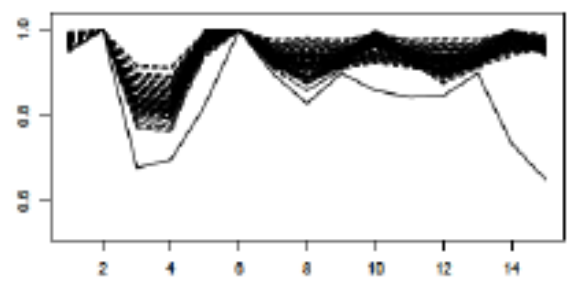

(c) Famila 3 con $n=1$

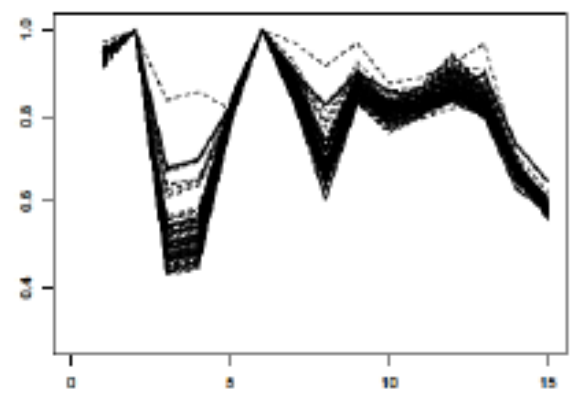

(e) Famila 4 con $n=1$

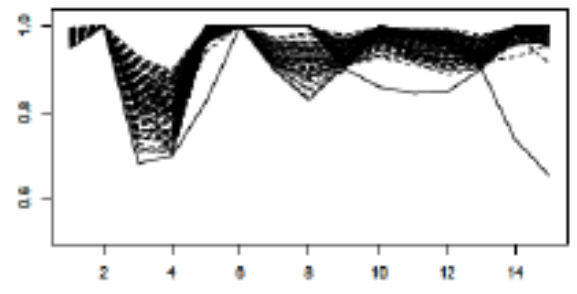

(b) Famila 2

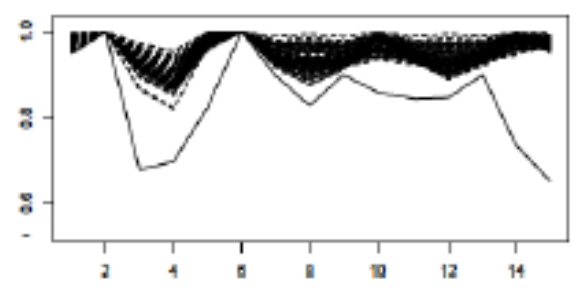

(d) Famila 3 con $n=2$

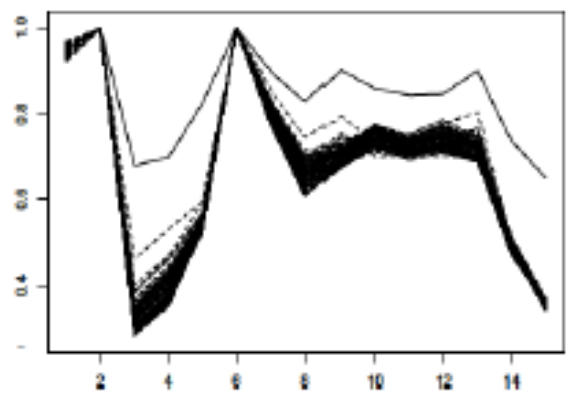

(f) Famila 4 con $n=2$

Figura 4.2.3: Comportamiento de las distintas familias de funciones de similitud

El ajuste al consenso es ligeramente peor para las funciones de la segunda y la tercera familias, y en caso de las funciones de la Familia 4 con $n=1$, el ajuste al consenso es muy superior a las otras. En la Figura 4.2.4 puede verse en trazo discontinuo la función de la familia 3 con los pesos $w_{1}=0.303185171, w_{2}=0.206263065, w_{3}=0.490551764, v_{1}=$ $0.240222879, v_{2}=0.162093729, v_{3}=0.159982925, v_{4}=0.433343553, v_{5}=0.433343553$. 


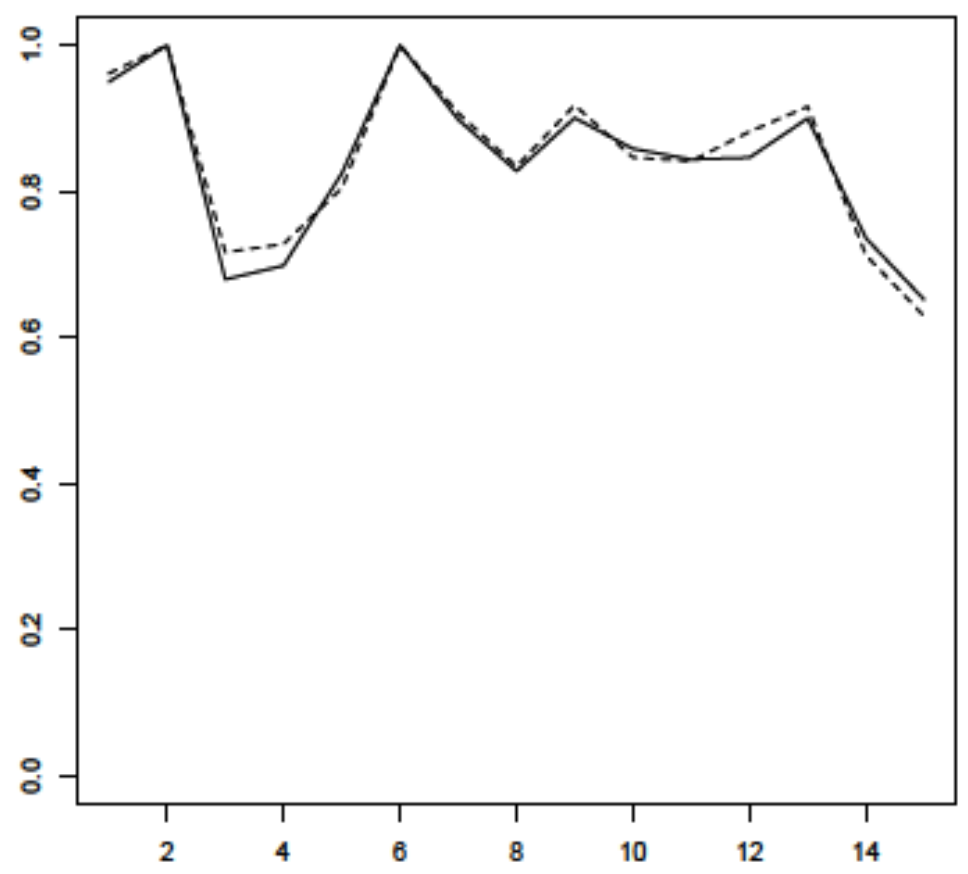

Figura 4.2.4: Aproximación de la media con una función de la Familia 4 con $n=1$

Parece claro que las funciones que mejor se ajustan al consenso son las de la Familia 4 con $n=1$. Nuestro objetivo entonces es encontrar la función de la Familia 4 con $n=1$ que más se aproxima al consenso.

En el caso del criterio de la media el problema se reduce a calcular los valores de $w_{1}, w_{2}, w_{3}, v_{1}, v_{2}, v_{3}, v_{4}, v_{5} \in[0,1]$ que minimizan la máxima diferencia entre $S\left(\widetilde{A}_{i j}, \widetilde{A}_{k l}\right) \mathrm{y}$ $\bar{S}_{i j}^{k l}$ :

$$
\begin{aligned}
& \text { Minimizar } \max _{i, j, k, l}\left\{\left|S\left(\widetilde{A}_{i j}, \widetilde{A}_{k l}\right)-\bar{S}_{i j}^{k l}\right|\right\} \\
& \text { s.a } \\
& \quad w_{1}+w_{2}+w_{3}=1,0 \leq w_{i} \leq 1 \quad \forall i \\
& \quad v_{1}+v_{2}+v_{3}+v_{4}+v_{5}=1,0 \leq v_{i} \leq 1 \quad \forall i
\end{aligned}
$$

donde

$$
\begin{aligned}
& S(\widetilde{A}, \widetilde{B})=\left[1-\left(w_{1} \frac{\sum\left|a_{i}-b_{i}\right|}{4}+w_{2} d\left(\left(X_{\widetilde{A}}, Y_{\widetilde{A}}\right),\left(X_{\widetilde{B}}, Y_{\widetilde{B}}\right)\right)+w_{3} \sqrt[4]{\prod_{i=1}^{4}\left|a_{i}-b_{i}\right|}\right)\right] \times \\
& \quad \times\left(\frac{v_{1} \min \left\{P_{\widetilde{A}}, P_{\widetilde{B}}\right\}+v_{2} \min \{A(\widetilde{A}), A(\widetilde{B})\}+v_{3} \min \left\{w_{\widetilde{A}}, w_{\widetilde{B}}\right\}+v_{4} \min \left\{Q_{\widetilde{A}}, Q_{\widetilde{B}}\right\}+v_{5} \int_{0}^{1} \mu_{\widetilde{A} \cap \widetilde{B}}(x) d x}{v_{1} \max \left\{P_{\widetilde{A}}, P_{\widetilde{B}}\right\}+v_{2} \max \{A(\widetilde{A}), A(\widetilde{B})\}+v_{3} \max \left\{w_{\widetilde{A}}, w_{\widetilde{B}}\right\}+v_{4} \max \left\{Q_{\widetilde{A}}, Q_{\widetilde{B}}\right\}+v_{5} \int_{0}^{1} \mu_{\widetilde{A} \cup \widetilde{B}}(x) d x}\right),
\end{aligned}
$$

es una función de la Familia 4 con $n=1 \mathrm{y} \bar{S}_{i j}^{k l}$ es el valor medio de todas las funciones de la literatura sobre el par $\left(\widetilde{A}_{k l}, \widetilde{A}_{i j}\right)$.

En el caso del coeficiente de correlación de Pearson, el problema se reduce a calcular 
los valores de $w_{1}, w_{2}, w_{3}, v_{1}, v_{2}, v_{3}, v_{4}, v_{5} \in[0,1]$ que maximizan el mínimo coeficiente de correlación $R_{i}$ entre la función $S$ de la Familia 4 con $n=1$ y cada una de las funciones de la literatura $S_{i}$ :

$$
\begin{aligned}
& \text { Maximizar } \min _{i}\left\{R_{i}\right\} \\
& \text { s.a } \\
& \qquad w_{1}+w_{2}+w_{3}=1,0 \leq w_{i} \leq 1 \forall i \\
& \quad v_{1}+v_{2}+v_{3}+v_{4}+v_{5}=1,0 \leq v_{i} \leq 1 \forall i .
\end{aligned}
$$

Estos problemas corresponden a modelos de regresión múltiple bastante complejos, con 8 variables de regresión que se calculan a partir de dos 5-uplas (los números borrosos trapezoidales generalizados) y, por tanto, son problemas de difícil tratamiento analítico, por lo que recurriremos a metaheurísticas ${ }^{1}$ para su resolución.

Por otro lado, conviene considerar un conjunto de pares de números borrosos trapezoidales generalizados más representativo que el conjunto de Chen y Chen [11]. Por ello, completaremos este conjunto con todos los pares numéricos encontrados en la literatura y que no pertenezcan al conjunto de Chen, evitando tomar números incorrectos como los ya referidos números de altura nula $(w=0)$ que como se ha indicado anteriormente, no tienen sentido por definición.

El conjunto completo de números encontrados en la literatura se muestra en la Tabla

\subsection{2.}

El conjunto de soluciones factibles está constituido por los hiperplanos $w_{1}+w_{2}+w_{3}=$ $1,0 \leq w_{i} \leq 1, \mathrm{y} v_{1}+v_{2}+v_{3}+v_{4}+v_{5}=1,0 \leq v_{i} \leq 1$, de $\mathbb{R}^{3}$ y $\mathbb{R}^{5}$ respectivamente, lo que dificulta el uso de metaheurísticas poblacionales.

Por ejemplo, si utilizamos algoritmos genéticos el operador de cruce generalmente lleva a individuos no aptos, y para la técnica de optimización por enjambre de partículas, al moverse cada partícula en la dirección de su velocidad generalmente se sale de los hiperplanos. Resultan más útiles las metaheurísticas trayectoriales que, partiendo de una o varias soluciones, busca en los entornos de las mismas restringiéndose a estos hiperplanos. Una de las metaheurísticas trayectoriales más utilizadas por su potencia y sencillez es el Recocido Simulado.

\footnotetext{
${ }^{1}$ En el Capítulo 6 se expone una introducción pormenorizada a estas técnicas de optimización.
} 
Tabla 4.2.2: Conjunto completo de pares de números borrosos para la comparación de funciones de similitud encontrados en la literatura

\begin{tabular}{|c|c|c|c|}
\hline Orden & $\mathrm{A}$ & $\mathrm{B}$ & Referenci: \\
\hline 1 & $(0.1,0.2,0.3,0.4 ; 1)$ & $(0.1,0.25,0.25,0.4 ; 1)$ & {$[55,76]$} \\
\hline 2 & $(0.2,0.3,0.4 ; 1)$ & $(0.1,0.2,0.3,0.4 ; 1)$ & 55,76 \\
\hline 3 & $(0.1,0.2,0.3,0.4 ; 1)$ & $(0.4,0.55,0.55,0.7 ; 1)$ & 55,76 \\
\hline 4 & $(0.1,0.2,0.3,0.4 ; 1)$ & $(0.4,0.5,0.6,0.7 ; 1)$ & 55,76 \\
\hline 5 & $(0.1,0.2,0.3,0.4 ; 1)$ & $(0.1,0.2,0.3,0.4 ; 0.8)$ & 55,76 \\
\hline 6 & $(0.3,0.3,0.3,0.3 ; 1)$ & $(0.3,0.3,0.3,0.3 ; 1)$ & 55,76 \\
\hline 7 & $(0.2,0.2,0.2,0.2 ; 1)$ & $(0.3,0.3,0.3,0.3 ; 1)$ & 55,76 \\
\hline 8 & $(0.1,0.2,0.2,0.3 ; 1)$ & $(0.3,0.3,0.3,0.3 ; 1)$ & 55,76 \\
\hline 9 & $(0.1,0.2,0.2,0.3 ; 1)$ & $(0.2,0.3,0.3,0.4 ; 1)$ & 55,76 \\
\hline 10 & $(0.1,0.4,0.4,0.7 ; 1)$ & $(0.3,0.4,0.4,0.5 ; 1)$ & 55,76 \\
\hline 11 & $(0.2,0.3,0.5,0.6 ; 1)$ & $(0.3,0.4,0.4,0.5 ; 1)$ & 55,76 \\
\hline 12 & $(0.4,0.4,0.4,0.8 ; 1)$ & $(0.3,0.4,0.4,0.5 ; 1)$ & 55,76 \\
\hline 13 & $(0.2,0.3,0.4,0.5 ; 1)$ & $(0.3,0.4,0.5,0.6 ; 1)$ & 55,76 \\
\hline 14 & $(0.1,0.2,0.2,0.3 ; 1)$ & $(0.1,0.2,0.2,0.3,0.7)$ & 55,76 \\
\hline 15 & $(0.1,0.2,0.3,0.4 ; 1)$ & $(0.2,0.2,0.2,0.2 ; 0.7)$ & 55,76 \\
\hline 16 & $(0.1,0.2,0.3,0.4 ; 4 / 7)$ & $(0.4,0.5,0.6,0.7 ; 4 / 7)$ & {$[55]$} \\
\hline 17 & $(0.1,0.2,0.3,0.4 ; 4 / 7)$ & $(0.55-2 \sqrt{3} / 9,0.55,0.55,0.55+2 \sqrt{3} / 9 ; 2 / 3)$ & {$[55]$} \\
\hline 18 & $(0,0.225,0,225,0.45 ; 0,225 \sqrt{3})$ & $(0.45,0.675,0.675,0.9 ; 0,225 \sqrt{3})$ & [55] \\
\hline 19 & $(0,0.225,0,225,0.45 ; 0,225 \sqrt{3})$ & $(0.675,0.675,0.675,0.675 ; 0,15 \sqrt{3})$ & [55] \\
\hline 20 & $(0,0.1,0.2,0.3 ; 1)$ & $(0.2,0.35,0.35,0.5 ; 1)$ & 55 \\
\hline 21 & $(0,0.15,0.15,0.3 ; 1)$ & $(0.2,0.3,0.4,0.5 ; 1)$ & 55 \\
\hline 22 & $(0,0.1,0.1,0.2 ; 1)$ & $(0.1,0.2,0.2,0.3 ; 1)$ & 55 \\
\hline 23 & $(0.7,0.8,0.8,0.9 ; 1)$ & $(0.8,0.9,0.9,1 ; 1)$ & 55 \\
\hline 24 & $(0,0.1,0.4,0.5 ; 1)$ & $(0.1,0.2,0.3,0.4 ; 1)$ & 55 \\
\hline 25 & $(0.5,0.6,0.9,1 ; 1)$ & $(0.6,0.7,0.8,0.9 ; 0.993942)$ & 55 \\
\hline 26 & $(0.1,0.2,0.3,0.4 ; 1)$ & $(0,0.1,0.1,0.2 ; 1)$ & 55 \\
\hline 27 & $(0.1,0.2,0.3,0.4 ; 1)$ & $(0.3,0.4,0.4,0.5 ; 1)$ & 55 \\
\hline 28 & $(0.1,0.1,0.2,0.3 ; 1)$ & $(0.2,0.3,0.4,0.4 ; 1)$ & 23 \\
\hline 29 & $(0.1,0.2,0.3,0.4 ; 1)$ & $(0.25,0.35,0.45,0.55 ; 1)$ & 23 \\
\hline 30 & $(0.1,0.1,0.2,0.3 ; 1)$ & $(0.2,0.3,0.4,0.4 ; 1)$ & 23 \\
\hline 31 & $(0.1,0.2,0.3,0.4 ; 1)$ & $(0.25,0.35,0.45,0.55 ; 1)$ & 23 \\
\hline 32 & $(0,0.1,0.4,0.5 ; 1)$ & $(0.1,0.2,0.3,0.4 ; 1)$ & 23 \\
\hline 33 & $(0,0.1,0.4,0.5 ; 1)$ & $(0.2,0.3,0.4,0.5 ; 1)$ & 23 \\
\hline 34 & $(0.1,0.2,0.3,0.4 ; 1)$ & $(0.5,0.65,0.65,0.8 ; 1)$ & 78 \\
\hline 35 & $(0.1,0.2,0.3,0.4 ; 1)$ & $(0.3,0.45,0.45,0.6 ; 1)$ & 78 \\
\hline 36 & $(0.1,0.2,0.3,0.4 ; 1)$ & $(0.1,0.25,0.25,0.4 ; 0.2)$ & 78 \\
\hline 37 & $(0.1,0.2,0.3,0.4 ; 1)$ & $(0.5,0.6,0.70 .8 ; 1)$ & 78 \\
\hline 38 & $(0.1,0.2,0.3,0.4 ; 1)$ & $(0.25,0.35,0.45,0.55 ; 1)$ & 78 \\
\hline 39 & $(0.1,0.2,0.3,0.4 ; 1)$ & $(0.3,0.4,0.5,0.6 ; 1)$ & 78 \\
\hline 40 & $(0.1,0.2,0.3,0.4 ; 1)$ & $(0.1,0.2,0.3,0.4 ; 0.8)$ & 78 \\
\hline 41 & $(0.1,0.2,0.3,0.4 ; 1)$ & $(0.3,0.3,0.3,0.3 ; 0.4)$ & 78 \\
\hline 42 & $(0.1,0.2,0.3,0.4 ; 0.8)$ & $(0.2,0.3,0.4,0.5 ; 0.6)$ & 78 \\
\hline 43 & $(0.1,0.25,0.25,0.4 ; 0.8)$ & $(0.1,0.25,0.25,0.4 ; 0.6)$ & 78 \\
\hline 44 & $(0.1,0.25,0.25,0.4 ; 0.8)$ & $(0.3,0.45,0.45,0.6 ; 0.8)$ & 78 \\
\hline 45 & $(0.1,0.25,0.25,0.4 ; 0.8)$ & $(0.3,0.3,0.3,0.3 ; 0.6)$ & 78 \\
\hline 46 & $(0.1,0.2,0.3,0.4 ; 1)$ & $(0.5,0.6,0.7,0.8 ; 1)$ & 78 \\
\hline 47 & $(0.4,0.4,0.4,0.4 ; 0.4)$ & $(0.5,0.5,0.5,0.5 ; 0.2)$ & 78 \\
\hline 48 & $(0.1,0.2,0.3,0.4 ; 1)$ & $(0.3,0.4,0.5,0.6 ; 1)$ & 78 \\
\hline
\end{tabular}


Analizaremos en detalle la metaheurística de recocido simulado en el Capítulo 6. Aquí, únicamente daremos su pseudocódigo para un problema de maximización:

- Generar una solución factible inicial $x_{0}$. Hacer $x^{*}=x_{0}, f^{*}=f\left(x_{0}\right), i=0$. Seleccionar un valor para la temperatura inicial $t_{0}=T\left(t_{i}\right.$ temperatura en el paso. $\left.i\right)$

- Repetir hasta satisfacer el criterio de parada:

- Generar aleatoriamente $y \in N\left(x_{i}\right)$ donde $x_{i}$ es la solución en el paso $i$.

- Si $f(y)-f\left(x_{i}\right) \geq 0$, entonces

$\circ x_{i+1}=y$.

○ Si $\left(f\left(x^{*}\right)>f\left(x_{i}\right)\right)$, entonces $x^{*}=x_{i}, f^{*}=f\left(x_{i}\right)$.

en otro caso:

$\circ p \sim U(0,1)$.

○ Si $p \leq e^{-\left(f(y)-f\left(x_{i}\right)\right) / t_{i}}$, entonces $x_{i+1}=y$.

- $i=i+1$.

- Actualizar temperatura.

Los elementos de recocido simulado son la solución inicial, la temperatura inicial, la forma de enfriamiento y el criterio de parada. La solución inicial se toma de manera aleatoria en los hiperplanos factibles.

La temperatura inicial se toma de modo que la probabilidad de aceptación de una vecina de la solución inicial sea al menos del $90 \%$, independientemente de lo mala que sea la solución vecina. Para ello, basta tomar una cota superior de $\left|f(y)-f\left(x_{0}\right)\right|$, ya que si $M>\left|f(y)-f\left(x_{0}\right)\right|$ entonces tomando $T_{0}=\frac{-M}{\ln (0.9)}$ se tiene que $\ln (0.9) \leq \frac{-\left(f(y)-f\left(x_{0}\right)\right)}{T_{0}} \Rightarrow$ $0.9 \leq e^{\frac{-\left(f(y)-f\left(x_{0}\right)\right)}{T_{0}}}$.

Para el criterio de consenso basado en la media una cota superior de $\left|f(y)-f\left(x_{0}\right)\right|$ es $M=1$. Para el criterio del coeficiente de correlación de Pearson, $M=2$. Por tanto la temperatura inicial debe tomarse en torno a 10 y 20 respectivamente.

La forma de enfriamiento en ambos casos consiste en reducir un 5\% la temperatura cada 20 iteraciones y el criterio de parada del algoritmo consiste en finalizar si la solución no ha mejorado en el último $30 \%$ del total de las iteraciones. 
En la Figura 4.2.5(a) puede verse la trayectoria de la función objetivo en la ejecución del recocido simulado en el caso de la media. La solución obtenida por este criterio es $w_{1}=$ $0.440350852, w_{2}=0.096370747, w_{3}=0.463278402, v_{1}=0.149429478, v_{2}=0.057426459$ $v_{3}=0.186965168, v_{4}=0.604668444$ y $v_{5}=0.001510451$. La máxima distancia a la media es de 0.1272644 .

En la Figura 4.2.6 puede verse el ajuste a la media dado por la función de consenso obtenida.

En la Figura 4.2.5(b) puede verse la trayectoria de la función objetivo en la ejecución del recocido simulado en el caso del criterio del coeficiente de correlación de Pearson. La solución obtenida por este criterio es $w_{1}=0.23397143, w_{2}=0.74421944, w_{3}=0.02180914$, $v_{1}=0.10937362, v_{2}=0.12299125 v_{3}=0.15032410, v_{4}=0.60450786$ y $v_{5}=0.01280318$. El coeficiente de correlación de Pearson de la solución encontrada con respecto a cada una de las funciones de similitud consideradas es Wei y Chen: 0.9613269, Chen: 0.9206363, Xu et al.: 0.9028064, Wen et al.: 0.9059533, Vicente et al.: 0.9626929, Sridevi and Nadarajan: 0.9457026 .

En las Figuras 4.2.7 y 4.2.8 comparamos gráficamente la funcion de similitud obtenida con este criterio y las seis funciones de similitud consideradas de la literatura.

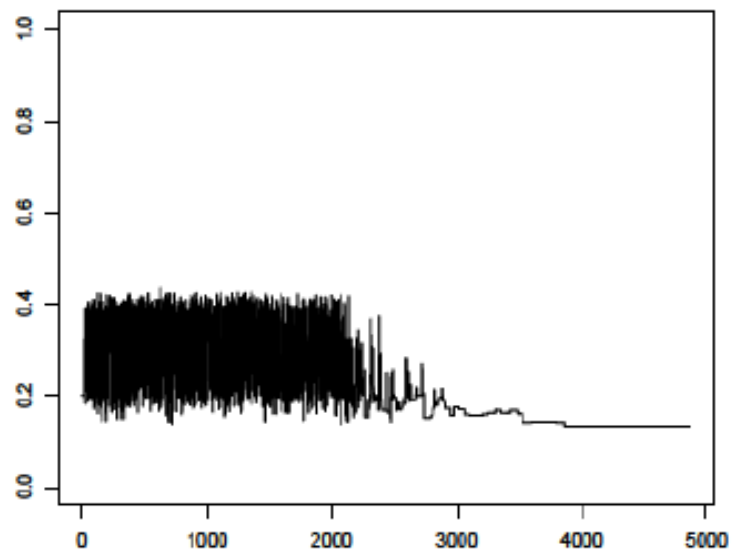

(a) Trayectoria de la función objetivo para el criterio de la media

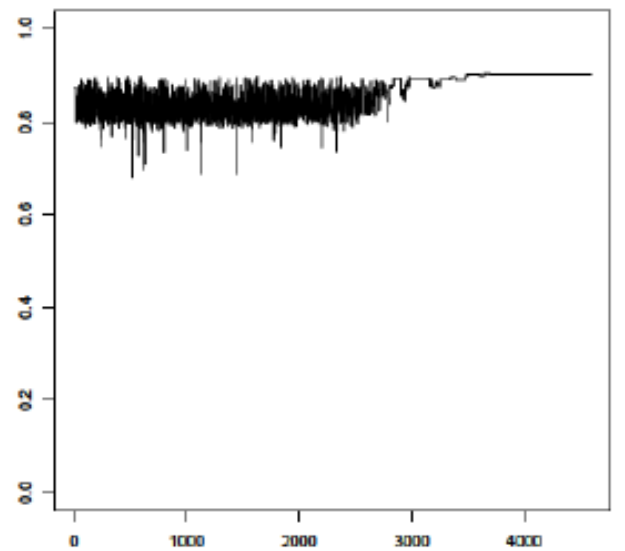

(b) Trayectoria de la función objetivo para el criterio de los coeficientes de correlación de Pearson

Figura 4.2.5: Trayectorias de las funciones objetivo para ambos consensos 


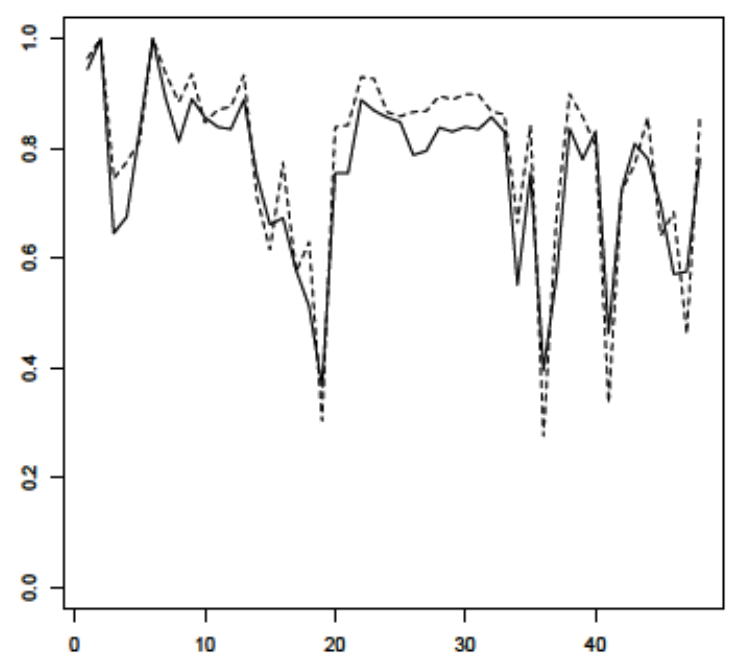

Figura 4.2.6: Ajuste a la media por la función de consenso

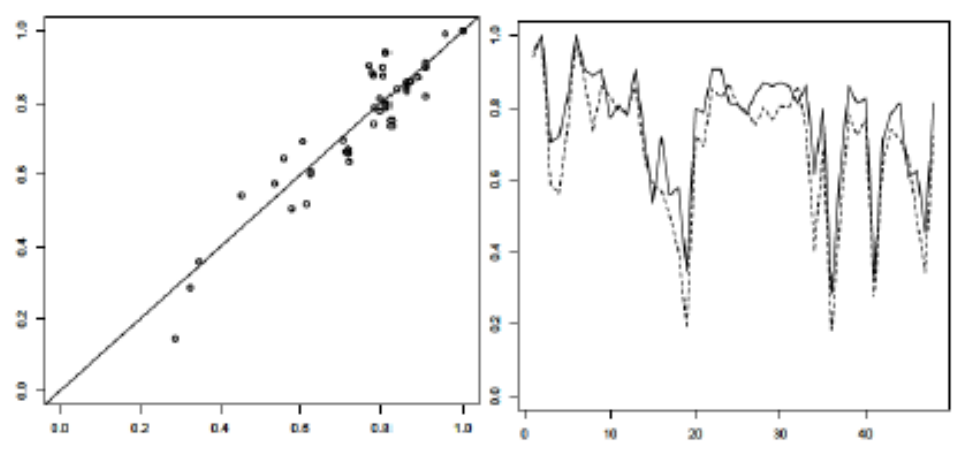

(a) Con la función de Sridevi y Nadarajan (2009) [55]

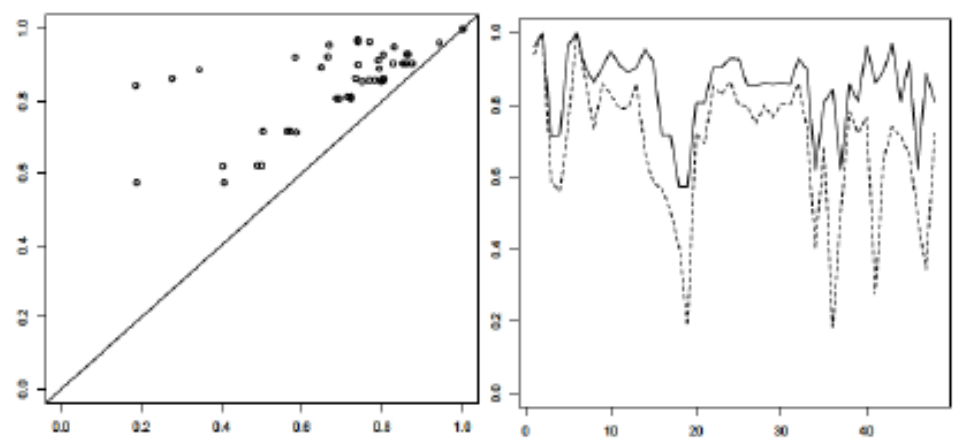

(b) Con la función de Xu et al. (2010) [78]

Figura 4.2.7: Comparación de la función de consenso de Pearson con el resto de funciones 


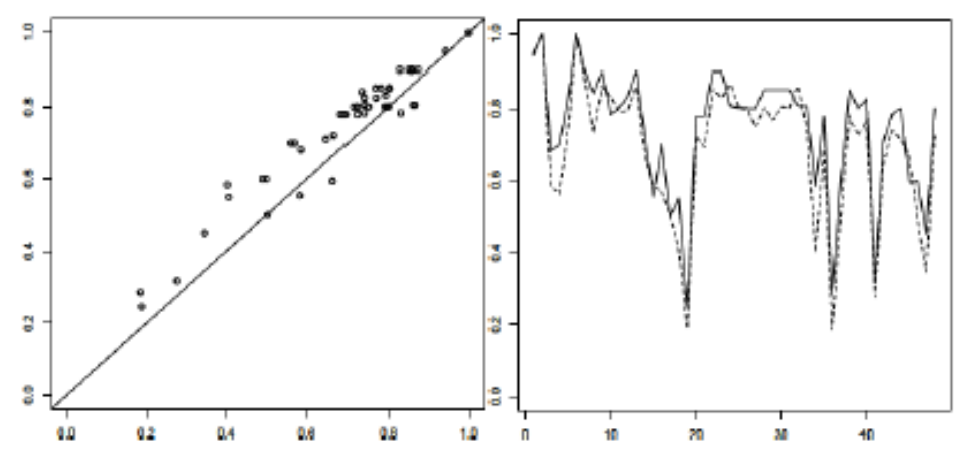

(a) Con la función de Wei y Chen (2009) [76]

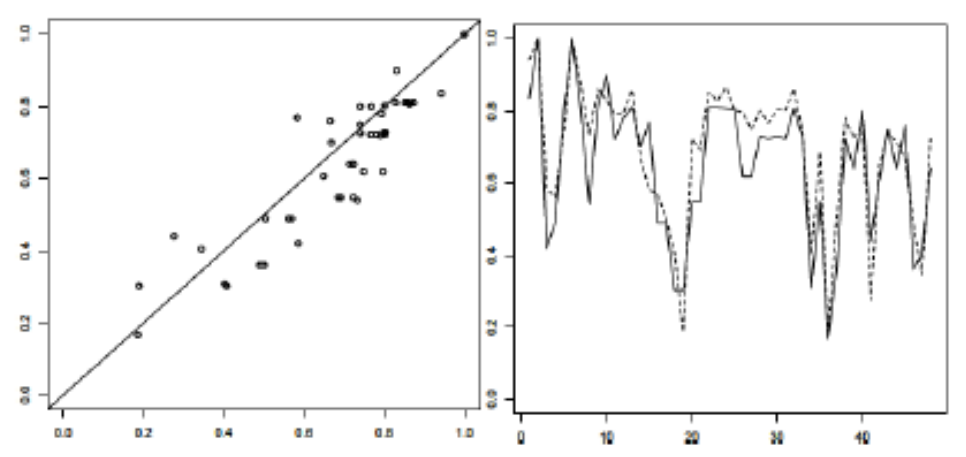

(b) Con la función de Chen y Chen (2003) [11]

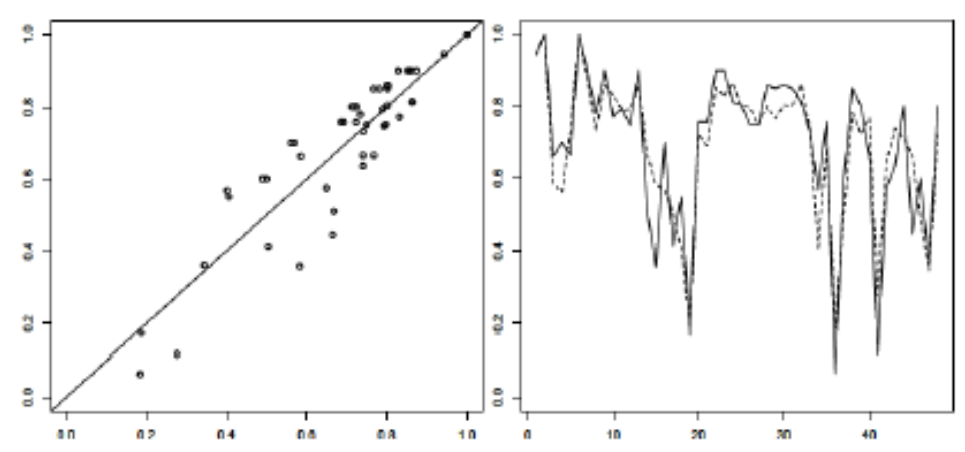

(c) Con la función de Wen et al (2013) [77]

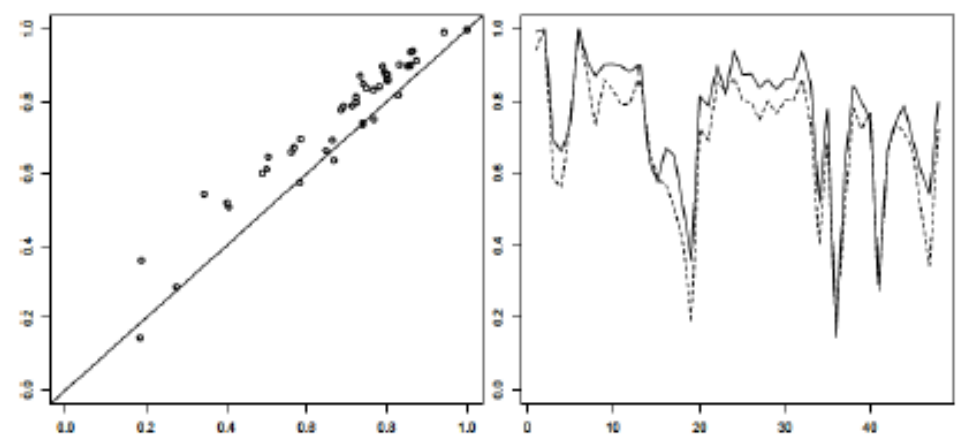

(d) Con la función de Vicente et al. (2013) [62]

Figura 4.2.8: Comparación de la función de consenso de Pearson con el resto de funciones (continuación) 


\subsubsection{Ventajas de la similitud de consenso}

Se han establecido dos funciones de similitud de consenso entre ocho funciones de similitud de números borrosos trapezoidales de la literatura. Estas funciones mejoran al resto de funciones de la literatura por las siguientes razones:

1. Las funciones propuestas tiene mejores propiedades que algunas funciones de la literatura, como la de Xu et al., que mide mal la similitud entre dos números reales, o la de Zhu y Xu que no está completamente definida.

2. Las funciones propuestas consideran todos los puntos de vista propuestos por el resto de las funciones de similitud y ante la gran variabilidad en cuanto a la medida de la similitud de un mismo número, se establece un necesario consenso.

3. El método desarrollado permite incorporar fácilmente nuevos puntos de vista (parámetros) de modo que puede mejorarse continuamente.

4. El método permite evaluar la necesidad de cada parámetro, de modo que podamos prescindir de aquéllos cuyos pesos sean muy reducidos y obtener por el mismo procedimiento una función refinada con menos parámetros. 


\section{Capítulo 5 \\ METODOLOGÍAS DE ANÁLISIS Y GESTIÓN DE RIESGOS EN LOS SISTEMAS DE INFORMACIÓN}

La organización Internacional de Normalización (ISO), formada por 163 países, y la Comisión Electrotécnica Internacional (IEC), formada por 60 países, son los organismos internacionales encargados de la normalización y estandarización internacional de productos y servicios para todas las ramas industriales.

En el ámbito de las Tecnologías de la Información y la Comunicación (TIC) ISO e IEC establecieron en 1987 un comité técnico conjunto (ISO/IEC JTC 1), formado por 35 países participantes y 56 países observadores, encargado de ofrecer las directivas estándar internacionales a cada uno de los organismos nacionales que lo integran. La publicación definitiva de un estándar internacional requiere de la aceptación de al menos un $75 \%$ de ellos [26].

Las normas internacionales que establecen los requisitos de certificación de los Sistemas de Gestión de Seguridad de la Información (SGSI) tienen su origen en la norma BS 7799 de la BSI (British Standards Institution). La primera parte de la norma (la BS 77991), publicada en 1995, establece por primera vez un conjunto de buenas prácticas para la gestión de seguridad de la información, para ser utilizado por cualquier empresa u organización, mientras que la segunda parte (BS 7799-2) de 1998 establece ya los requisitos de un sistema de un SGSI para ser certificable por auditorías independientes [8].

En 1999 el comité ISO/IEC JTC 1 acogió la BS 7799-1 sin cambios transcendentes como la ISO/IEC 17799 que en 2005 pasaría a llamarse ISO/IEC 27002, mientras que la norma BS 7799-2 sería adoptada como ISO/IEC 27001. Ésta es la norma fundamental del 
conjunto de estándares ISO/IEC 27000 que, en los rangos de numeración 27000 a 27019 y 27030 a 27044, proporciona el marco de gestión de seguridad de la información que sirve de base a adaptaciones nacionales o corporativas y a las diferentes metodologías de análisis y gestión de riesgos de los sistemas de información [8]

A lo largo de este capítulo, describiremos a grandes rasgos estas metodologías para aportar posteriormente un marco teórico a partir del cual puedan desarrollarse herramientas tecnológicas que faciliten el proceso de análisis y gestión del riesgo.

\subsection{El ciclo de mejora continua}

El ciclo PDCA (Plan-Do-Check-Act) de mejora continua, o ciclo demming, es un modelo tradicionalmente usado en los sistemas de gestión de calidad y particularmente es adoptado también por las metodologías internacionales de la serie ISO/IEC 27000 que regulan los Sistemas de Gestión de Seguridad de la Información (SGSI). El modelo consta de cuatro fases (planificar, hacer, verificar y actuar) que permiten establecer un sistema de indicadores y métricas comparables en el tiempo de manera que se pueda cuantificar el avance en la mejora de la organización [24]. Un esquema adaptado a los SGSI es [52]:

1. Planificar (establecimiento del SGSI).

- Definir el alcance del SGSI y la política de seguridad de la organización.

- Identificar y evaluar los riesgos.

- Seleccionar los objetivos de control y los controles que ayudan a controlar estos riesgos.

- Preparar la Declaración de Aplicabilidad, documentando los controles seleccionados y justificando dichas decisiones.

En esta fase es necesario establecer un modelo organizativo (Figura 5.1.1) cuya figura central será el Comité de Seguridad, máximo órgano decisor del SGSI [7] integrado por todas las áreas implicadas en la seguridad de la información de la organización. Dicho comité será el encargado de seleccionar a los responsables de desarrollar el SGSI, proveer los medios necesarios y planificar el proceso de establecimiento del SGSI. 


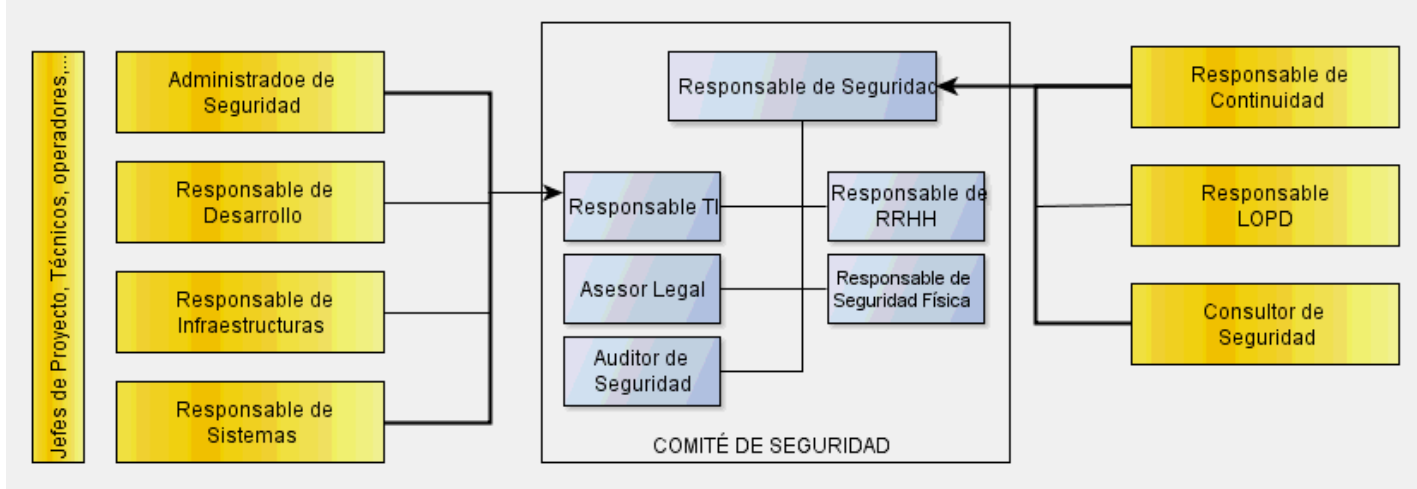

Figura 5.1.1: Modelo Organizativo del SGSI

Como puede observarse, la actividad central en esta fase es el análisis de riesgos y la selección de controles que permitan mitigar esos riesgos (en la Sección 5.1.2 veremos con mayor rigor los conceptos de control y objetivos de control). El análisis de riesgos recomendado por la norma ISO/IEC 27001 consiste en identificar una metodología de análisis que lleve al establecimiento de los activos, las vulnerabilidades, las amenazas y la salvaguardas que se deben tener en consideración para el buen funcionamiento de la organización.

2. Hacer (implementación y puesta en funcionamiento).

- Formular e implementar un plan de mitigación de riesgos.

- Implementar los controles previamente seleccionados para cumplir con los objetivos de control.

Una vez que se ha realizado el análisis de riesgos y la selección de controles es necesario poner en funcionamiento las acciones prescritas en los controles en el día a día de la organización.

3. Verificar (monitorización y revisión del SGSI).

- Llevar a cabo revisiones periódicas para verificar la eficacia del SGSI.

- Revisar los niveles de riesgo aceptable y residual.

- Periódicamente realizar auditorías internas del SGSI. 
Es fundamental registrar y observar cada incidencia en el funcionamiento del SGSI y de los propios activos de la organización con el fin de aprender e identificar posibilidades de mejora.

4. Actuar (mantenimiento y mejora del SGSI).

- Implementar las mejoras identificadas en el SGSI.

- Tomar medidas correctivas y preventivas.

- Mantener la comunicación con todas las partes interesadas.

- Validar las mejoras.

Pasado un tiempo razonable de aprendizaje del sistema es preciso poner en funcionamiento las posibilidades de mejoras identificadas en la fase anterior enlazando de nuevo con la fase Plan con un SGSI mejorado.

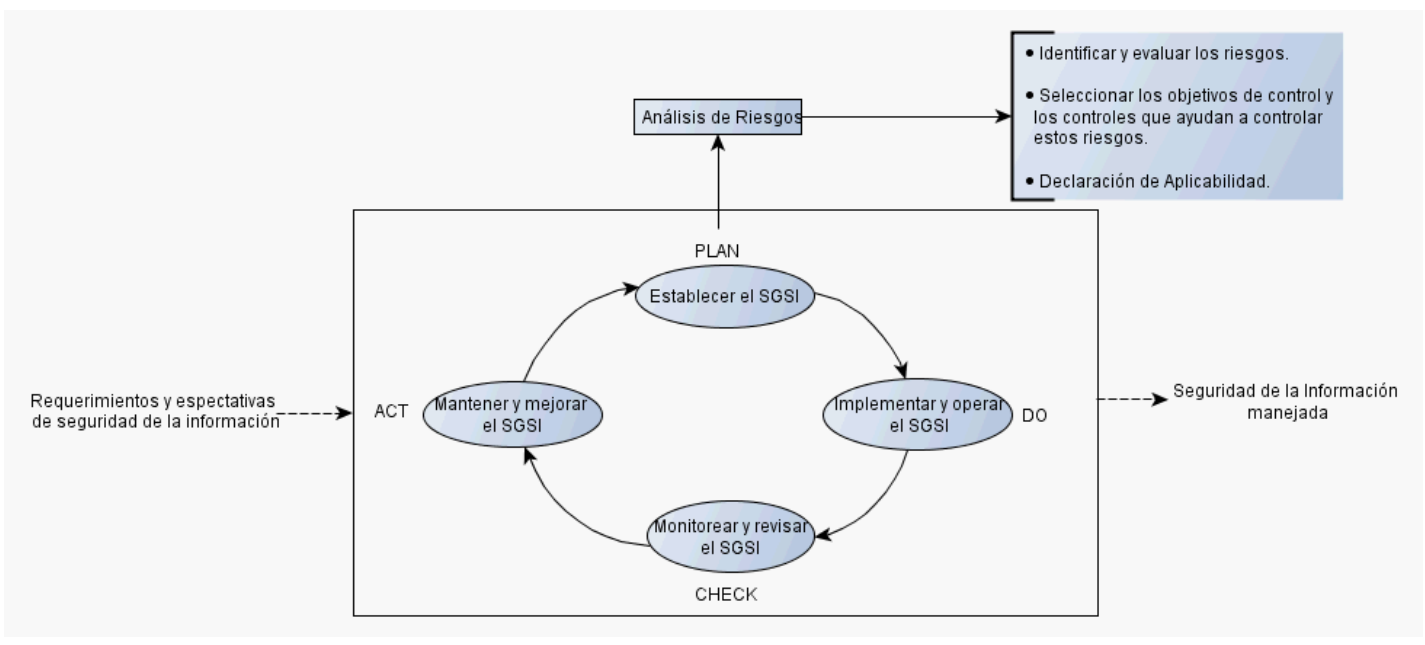

Figura 5.1.2: Ciclo de mejora continua

De este modelo de mejora continua conviene señalar que es un esfuerzo innecesario partir de un SGSI perfecto. El objetivo deberá ser [24] diseñar un SGSI que se ajuste lo más posible a la organización en el momento de inicio y que consuma pocos recursos, contemplando las medidas de seguridad mínimas para proteger la información y cumplir con la norma. El SGSI evolucionará a lo largo del tiempo gracias al aprendizaje del conjunto del modelo organizativo encargado de la seguridad de la información (comité de seguridad y responsables del desarrollo del SGSI). 
Volveremos sobre el modelo PDCA en la Sección 5.4, en la que concretaremos dicho modelo según la norma ISO/IEC 27001.

\subsection{La serie ISO/IEC 27000}

Como hemos indicado anteriormente, la serie ISO/IEC 27000 está reservada a la seguridad de la información de las organizaciones. Conviene describir algunas de estas normas:

- ISO/IEC 27000:2009, Information security management systems Overview and vocabulary [28]: Este estándar ofrece una visión general de la familia de las normas de SGSI, una introducción a los SGSI, los términos y definiciones utilizados en la familia de estándares ISO/IEC 27000 y una breve descripción del modelo PDCA.

- ISO/IEC 27001:2005, Information security management systems Requirements [27]. Como se ha dicho anteriormente, es la norma principal de la serie. El objetivo de la norma en sí misma es "proporcionar un modelo para establecer, implementar, operar, supervisar, revisar, mantener y mejorar un SGSI [27]". La norma explica cómo diseñar un SGSI y establecer los controles de seguridad de acuerdo con las necesidades de una organización o de partes de la misma, pero no aclara mediante qué procedimientos se ponen en práctica [24].

Por ejemplo, uno de los principales requisitos es la realización de un análisis de riesgos con unas determinadas características de objetividad y precisión, pero no aporta indicaciones de cuál es la mejor manera de llevar a cabo dicho análisis.

Puede ejecutarse con una herramienta comercial, con una aplicación diseñada expresamente para la empresa, mediante reuniones, entrevistas, tablas o cualquier otro método que se estime oportuno [24].

- ISO/IEC 27002:2005, Code of practice for information security management [26]: 
Es una guía de buenas prácticas estructurada en 11 cláusulas de control de seguridad, cada una de las cuales contiene varias categorías principales (39 en total) que contienen un objetivo de control cada una y uno o varios controles (133 en total).

Los objetivos de control establecen lo que se debería lograr [26], mientras que los controles son los medios para manejar el riesgo y que se pueden aplicar para lograr el objetivo de control; incluyendo políticas, procedimientos, lineamientos, prácticas o estructuras organizacionales, las cuales pueden ser administrativas, técnicas, de gestión o de naturaleza legal [26]. El término "control" es sinónimo de salvaguarda o contramedida.

Las once cláusulas de control son:

a) Política de seguridad.

b) Organización de la seguridad de la información.

c) Gestión de activos.

d) Seguridad de recursos humanos.

e) Seguridad física y ambiental.

f) Gestión de comunicaciones y operaciones.

g) Control de acceso.

h) Adquisición, desarrollo y mantenimiento de sistemas de información.

i) Gestión de incidentes de seguridad de la información.

j) Gestión de la continuidad comercial.

k) Cumplimiento.

Para cada control se establecen una serie de guías (buenas prácticas recomendadas) para su implementación. Las organizaciones deben decidir el grado de implementación de cada uno de estos controles en función de sus necesidades de seguridad y los recursos de que disponga [24].

- ISO/IEC 27003:2010, Information security management system implementation guidance [29]: El propósito de esta norma es proporcionar orientación práctica en el desarrollo del plan de implementación de un SGSI 
dentro de una organización de acuerdo con ISO / IEC 27001:2005.

\subsection{Términos y definiciones}

Como se ha indicado, la serie ISO/IEC 27000 comienza en la norma ISO/IEC 27000:2009 con una introducción y un glosario de términos a utilizar en el SGSI. A continuación, se destacan algunos término útiles en esta tesis.

Activo: Cualquier cosa que tenga valor para la organización (ISO/IEC 13335$1: 2004)$.

Control: Medios para manejar el riesgo; incluyendo políticas, procedimientos, lineamientos, prácticas o estructuras organizacionales, las cuales pueden ser administrativas, técnicas, de gestión o de naturaleza legal.

NOTA. El control también se utiliza como sinónimo de salvaguarda o contramedida.

Lineamiento: Una descripción que aclara qué se debiera hacer y cómo, para lograr los objetivos establecidos en las políticas (ISO/IEC 13335-1:2004).

Medios de procesamiento de la información: Cualquier sistema, servicio o infraestructura de procesamiento de la información, o los locales físicos que los alojan.

Seguridad de la información: Preservación de confidencialidad, integración y disponibilidad de la información; Además, también puede involucrar otras propiedades como autenticidad, responsabilidad y confiabilidad.

Evento de seguridad de la información: Cualquier evento de seguridad de la información es una ocurrencia identificada del estado de un sistema, servicio o red indicando un posible fallo en la política de seguridad de la información o una situación previamente desconocida que puede ser relevante para la seguridad. (ISO/IEC TR 18044:2004).

Incidente de seguridad de la información: Un incidente de seguridad de la información es indicado por un solo evento o una serie de eventos inesperados de seguridad de la información que tienen una probabilidad significativa de comprometer las operaciones comerciales y amenazar la seguridad de la información. (ISO/IEC TR 18044:2004). 
Política de seguridad: Intención y dirección general expresada formalmente por la gerencia.

Riesgo: Combinación de la probabilidad de un evento y su ocurrencia (ISO/IEC Guía 73:2002).

Análisis del riesgo: Uso sistemático de la información para identificar las fuentes y calcular el riesgo.

Evaluación del riesgo: Proceso de comparar el riesgo estimado con un criterio de riesgo dado para determinar la importancia del riesgo (ISO/IEC Guía 73: 2002).

Gestión del riesgo: Actividades coordinadas para dirigir y controlar una organización con relación al riesgo. NOTA. La gestión del riesgo normalmente incluye la evaluación del riesgo, tratamiento del riesgo, aceptación del riesgo y comunicación del riesgo. (ISO/IEC Guía 73: 2002).

Tratamiento del riesgo: Proceso de selección e implementación de medidas para modificar el riesgo. (ISO/IEC Guía 73: 2002).

Amenaza: Una causa potencial de un incidente no deseado, el cual puede resultar en daño a un sistema u organización (ISO/IEC 13335-1:2004).

Vulnerabilidad: La debilidad de un activo o grupo de activos que puede ser explotada por una o más amenazas. (ISO/IEC 13335-1:2004).

\subsection{La norma ISO/IEC 27001.}

Como decíamos anteriormente, la ISO/IEC 27001 es la norma fundamental de la serie 27000 encargada de regular los sistemas de gestión de la seguridad de la Información. Esta norma concreta el modelo PDCA que se puede resumir en la Tabla $5.4 .1[27]$. 
Tabla 5.4.1: Resumen del Modelo PDCA de la ISO/IEC 27001

\begin{tabular}{|c|c|}
\hline Planear & $\begin{array}{l}\text { Establecer políticas, objetivos procesos y procedimientos del SGSI } \\
\text { relevantes para manejar el riesgo y mejorar la seguridad de la } \\
\text { información para entregar resultados en concordancia con las } \\
\text { políticas y objetivos generales de la organización. }\end{array}$ \\
\hline Hacer & $\begin{array}{l}\text { Implementar y operar la política, controles, procesos } \\
\text { y procedimientos. }\end{array}$ \\
\hline Chequear & $\begin{array}{l}\text { Evaluar y medir el desempeño del proceso de comparación } \\
\text { con la política, objetivos y experiencias prácticas del SGSI, y } \\
\text { reportar los resultados a la gerencia para su revisión. }\end{array}$ \\
\hline Actuar & $\begin{array}{l}\text { Tomar acciones correctivas y preventivas, basadas en los } \\
\text { resultados y en la auditoría interna del SGSI y la revisión } \\
\text { gerencial u otra información relevante para lograr la mejora } \\
\text { continua del SGSI. }\end{array}$ \\
\hline
\end{tabular}

A continuación, describimos con mayor profundidad cada una de estas fases.

\section{PLANIFICAR (Establecer y manejar el SGSI)}

La organización debe hacer lo siguiente [27]:

a) Definir el alcance del SGSI en términos de las características del negocio, su ubicación, activos, tecnología,...

b) Definir una política SGSI en términos de las características del negocio, su ubicación, activos, tecnología,... que:

1) incluya un marco referencial para establecer sus objetivos y una dirección y principios para la acción con relación a la seguridad de la información.

2) tenga en cuenta los requerimientos comerciales y legales o reguladores, y las obligaciones de la seguridad contractual.

3) esté alineada con el contexto de la gestión del riesgo estratégico de la organización en el cual se dará el establecimiento y mantenimiento del SGSI.

4) establece el criterio con el que se evaluará el riesgo.

5) haya sido aprobada por la gerencia. 
c) Definir el enfoque de la evaluación del riesgo de la organización:

1) Identificar una metodología de cálculo del riesgo adecuada para el SGSI y los requerimientos identificados de seguridad, legales y reguladores de la información.

2) Desarrollar los criterios para aceptar los riesgos e identificar los niveles de riesgo aceptables.

La metodología de estimación del riesgo seleccionada debe asegurar que los cálculos de riesgos produzcan resultados comparables y reproducibles.

Existen diferentes metodologías para el análisis de riesgos recogidas en ISO/IEC TR 13335-3 [30] y en ISO/IEC 27005 [31].

d) Identificar los riesgos:

1) Identificar los activos dentro del alcance del SGSI y los responsables de estos activos.

2) Identificar las amenazas para aquellos activos.

3) Identificar las vulnerabilidades que podrían ser explotadas por las amenazas.

4) Identificar los impactos que pueden tener las pérdidas de confiabilidad, integridad y disponibilidad sobre los activos.

e) Analizar y evaluar el riesgo:

1) Cacular el impacto sobre la organización que podría conllevar un fallo en la seguridad.

2) Calcular la probabilidad realista de que se materialicen estos fallos.

3) Calcular los niveles de riesgo.

4) Determinar si el riesgo es aceptable o requiere tratamiento.

f) Identificar y evaluar las opciones para el tratamiento de los riesgos.

1) Aplicar los controles apropiados.

2) Aceptar los riesgos siempre que satisfagan los criterios de aceptación.

3) Evitar los riesgos inaceptables. 
4) Transferir los riesgos a otras entidades, si se considera oportuno, por ejemplo, aseguradoras y proveedores. Hay que tener en cuenta que esto no exime de responsabilidades a la organización.

Los controles deben ser seleccionados de entre los que conforman el Anexo $A$ de la norma, que también aparecen en la norma ISO/IEC 27002.

g) Obtener la aprobación de la gerencia para los riesgos residuales (riesgos existente tras la implementación de controles) propuestos.

h) Obtener la aprobación de la gerencia para implementar y operar el SGSI.

i) Preparar una declaración de aplicabilidad:

1) Los objetivos de control y los controles seleccionados y las razones de su selección.

2) Los objetivos de control y los controles implementados actualmente.

3) La exclusión de cualquier control y objetivo de control del Anexo A de la norma ISO/IEC 27001 y su justificación.

\section{HACER (Implementar y operar el SGSI)}

Una vez definidos los controles que debemos implementar en la gestión de seguridad de la información, es necesario describir y recoger documentalmente cómo se van a desarrollar dichas medidas en el día a día de la organización, así como definir un conjunto de métricas para medir la efectividad de los controles implementados a lo largo del tiempo y comparar los resultados.

La organización debe hacer lo siguiente [27]:

a) Formular un plan de tratamiento de riesgo que identifique la acción gerencial apropiada, los recursos, las responsabilidades y prioridades para manejar los riesgos de la seguridad de la información.

b) Implementar el plan de tratamiento de riesgo para poder lograr los objetivos de control identificados, teniendo en consideración la financiación y asignación de roles y responsabilidades. 
c) Implementar los controles seleccionados para satisfacer los objetivos de control.

d) Definir cómo medir la efectividad de los controles o grupos de controles seleccionados y especificar cómo se van a utilizar tras estas mediciones para evaluar la efectividad del control para producir resultados comparables y reproducibles.

e) Implementar los programas de capacitación y conocimiento.

f) Manejar las operaciones del SGSI.

g) Manejar recursos para el SGSI.

h) Implementar los procedimientos y otros controles capaces de permitir una pronta detección y respuesta a incidentes de seguridad.

\section{CHEQUEAR (Monitorear y revisar el SGSI)}

Es fundamental analizar periódicamente el desarrollo del SGSI a través de revisiones y auditorías internas, ya que las condiciones de contorno del sistema cambian constantemente a lo largo del tiempo. Además, es necesario mantener un registro de incidencias que permita por un lado vigilar el sistema de información de la organización, y por otro aprender de los errores cometidos.

La organización debe hacer lo siguiente [27]:

a) Ejecutar procedimientos de seguimiento y revisión, y otros controles para:

1) Detectar rápidamente los errores en los resultados de procedimiento.

2) Identificar rápidamente los incidentes y violaciones de seguridad fallidos y exitosos.

3) Permitir a la gerencia determinar si las actividades de seguridad delegadas a las personas o implementadas mediante la tecnología de la información se están realizando como se esperaba.

4) Ayudar a detectar los eventos de seguridad, evitando así los incidentes de seguridad mediante el uso de indicadores.

5) Determinar si son efectivas las acciones tomadas para resolver una violación de seguridad. 
b) Realizar revisiones regulares de efectividad del SGSI (incluyendo satisfacer la política y objetivos de seguridad del SGSI, y revisar los controles de seguridad) teniendo en cuenta los resultados de auditorías de seguridad, incidentes, mediciones de seguridad, sugerencias y retroalimentación de todas las partes interesadas.

c) Medir la efectividad de los controles para verificar que se hayan cumplido los requerimientos de seguridad.

d) Revisar las evaluaciones del riesgo a intervalos planeados y revisar el nivel de riesgo residual y riesgo aceptable identificado, teniendo en cuenta los cambios en:

1) La organización.

2) Tecnología.

3) Objetivos y procesos comerciales.

4) Amenazas identificadas.

5) Efectividad de los controles implementados.

6) Eventos externos como cambios en el ambiente legal o regulador, en obligaciones contractuales, clima social,...

e) Realizar auditorías internas del SGSI a intervalos planificados.

f) Realizar una revisión gerencial del SGSI sobre una base regular para asegurar que el alcance permanezca adecuado y se identifiquen las mejoras en el SGSI.

g) Actualizar los planes de seguridad para tener en cuenta los descubrimientos de las actividades de monitoreo y revisión.

h) Registrar las acciones y eventos que podrían tener un impacto sobre la efectividad o desempeño del SGSI.

\section{ACTUAR (Mantener y mejorar el SGSI)}

Como hemos indicado anteriormente, las condiciones que rodean al sistema de información cambian continuamente, de modo que el SGSI no puede ser entendido como un sistema estático y permanente. Las posibilidades de mejora aprendidas en 
la fase anterior deben considerarse en un SGSI renovado, cerrando el ciclo de mejora continua.

La organización debe realizar regularmente lo siguiente [27]:

a) Implementar las mejoras identificadas en el SGSI.

b) Tomar acciones correctivas y preventivas apropiadas. Aplicar lo aprendido de las experiencias de seguridad de otras organizaciones y aquéllas de la organización misma.

c) Comunicar los resultados y acciones a todas las partes interesadas con un nivel de detalle apropiado de acuerdo a las circunstancias y, cuando sea relevante, acordar cómo proceder.

d) Asegurar que las mejoras logren los objetivos señalados.

\subsection{Análisis y gestión de riesgos en los sistemas de gestión de seguridad de la información. La nor- ma ISO/IEC 27005}

Como vemos, el análisis de riesgos es la parte central del SGSI incluida en la fase Plan del Modelo de Mejora Continua. Las metodologías recomendadas por las normas de la serie ISO 27000 se recogen en los technical reports ISO/IEC TR 13335-3 e ISO/IEC TR 13335-4. Estas normas fueron sustituidas (y ampliadas) en 2008 por la ISO/IEC 27005. Los procesos de análisis y gestión de riesgos dados por esta norma se pueden consultar en la Figura 5.5.1

Los procesos centrales pueden verse con mayor detalle en la Figura 5.5.2, según hemos visto en la Sección anterior (en descripción de la fase Planificación del la norma ISO/IEC 27001).

Estos procesos son detallados en la norma ISO/IEC 27005. A continuación, describimos las dos primeras fases del Análisis y Gestión de Riesgos. 


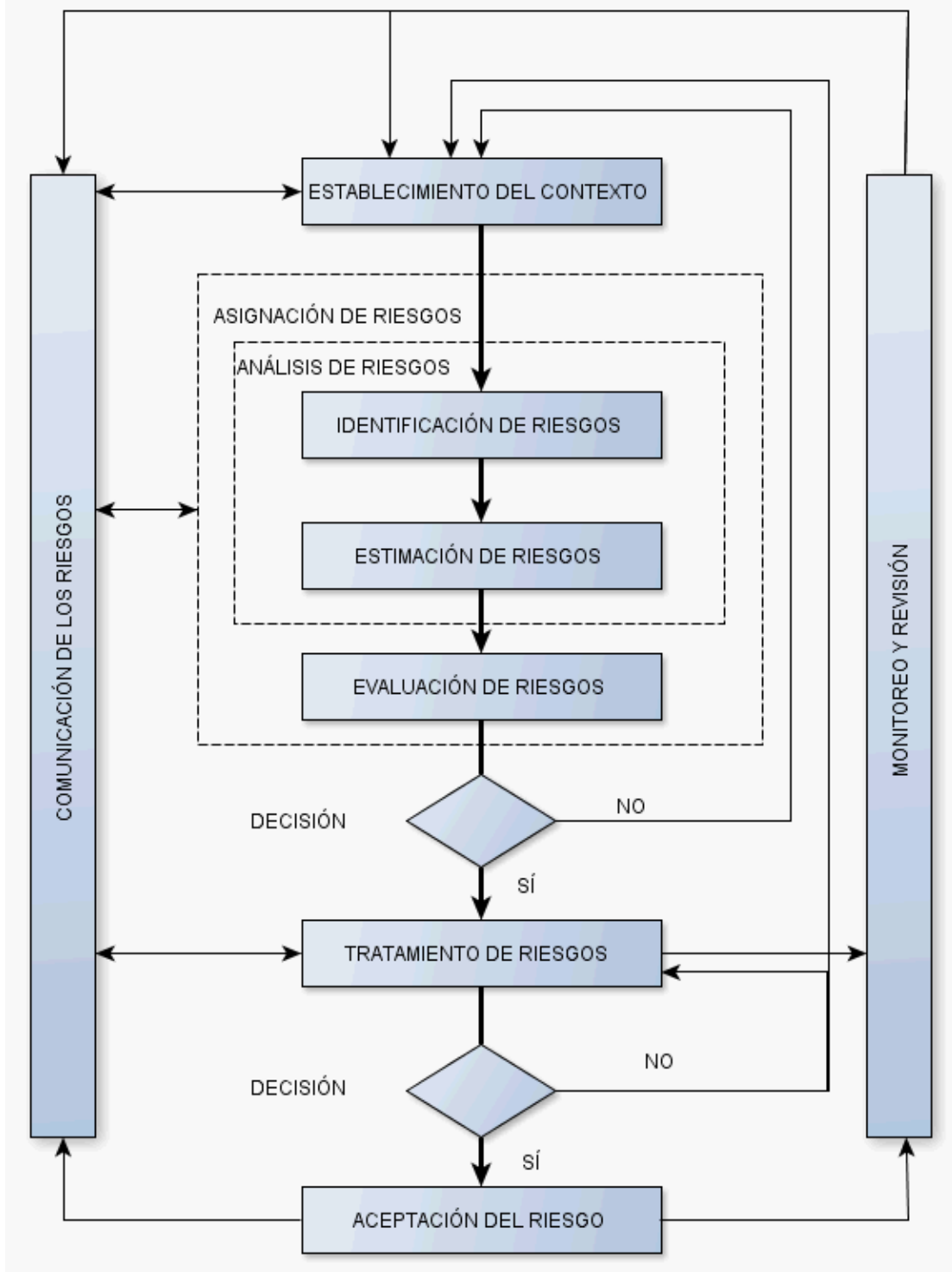

Figura 5.5.1: Esquema de procesos de análisis y gestión de riesgos en SI

\subsubsection{Identificación y valoración de los riesgos}

El propósito de la identificación de riesgos consiste en determinar las pérdidas potenciales (impactos) que se podrían dar en la organización si las amenazas que se tienen sobre los elementos de la misma llegaran a materializarse.

Una vez identificados estos riesgos, es necesario valorar tanto los elementos que influyen sobre el sistema de información (activos) como las amenazas que se ciernen sobre ellos, así como las vulnerabilidades de dichos activos.

Finalmente, estableceremos indicadores de impacto y riesgo sobre el sistema de 


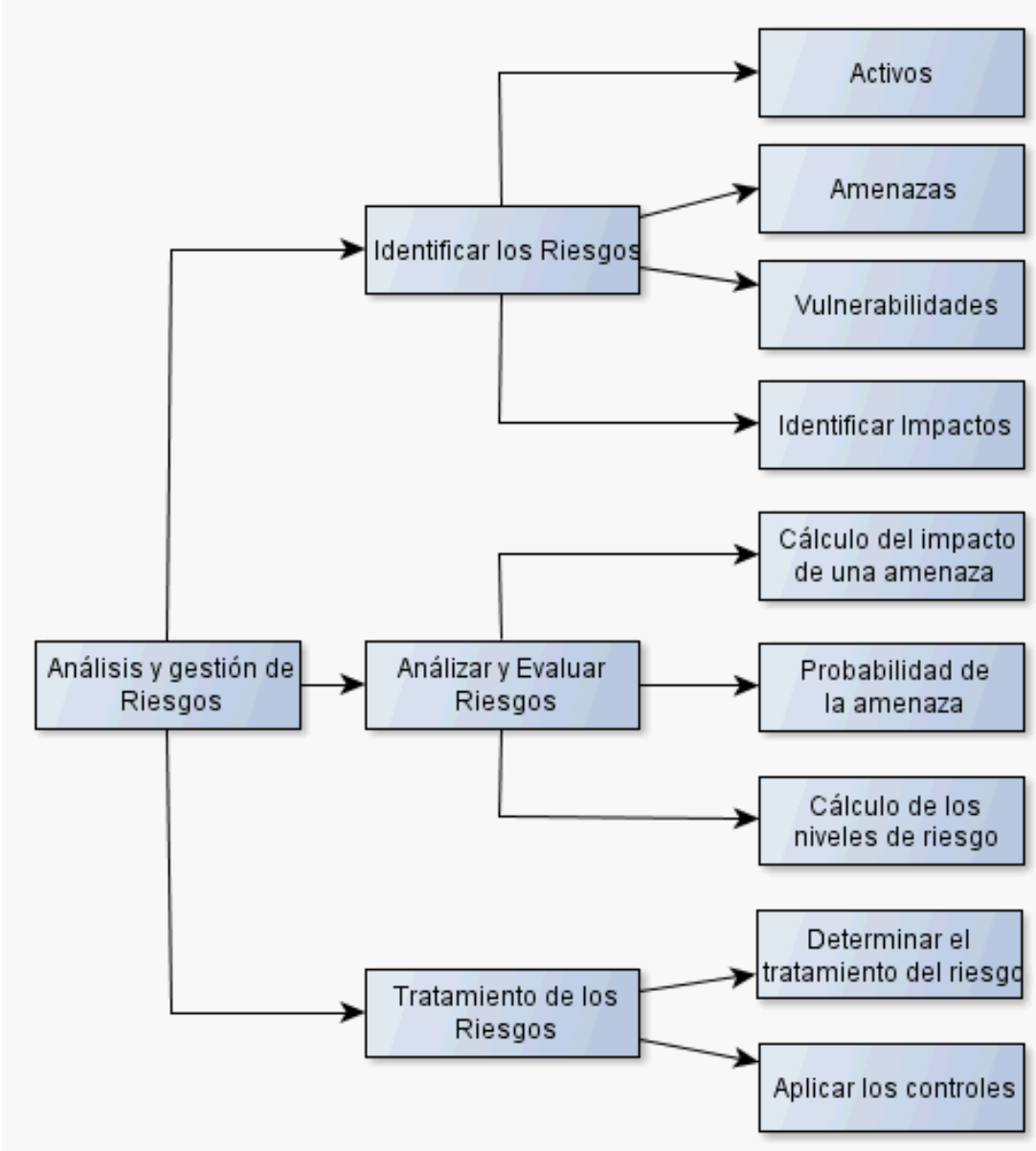

Figura 5.5.2: Procesos centrales del Análisis y Gestión de Riesgos de los SGSI (ISO/IEC 27001)

información.

\section{Identificación y valoración de los Activos}

Un activo es cualquier cosa que tenga valor para la organización y que, por lo tanto, requiere protección [31].

El valor total de los activos de una organización se suele concentrar en unos pocos activos de tipo datos, información o procesos de negocio. A estos activos los llamaremos Activos Primarios [31] o Activos Terminales [37]. El valor del resto de los activos (activos de soporte [31] como hardware, software, personal, instalaciones,...) radica en las prestaciones que dan a los activos primarios y, por tanto, heredan el valor de los activos primarios, según su influencia sobre éstos. Es decir, si el fallo de un determinado activo de soporte implica, por ejemplo, la total indisponibilidad de 
los procesos de negocio (activo primario), entonces el valor de este activo de soporte será muy alto (quizás tan alto como el del activo primario), pero si un fallo en el activo de soporte no se ve reflejado gravemente en los activos primarios, entonces el valor del activo de soporte no debe ser muy alto.

Es fundamental, por tanto, la identificación de los activos primarios y los activos de soporte de la organización. En el Anexo B de la norma ISO/IEC 27005, se proporcionan listas detalladas de los activos más comunes a las organizaciones en cuanto a los sistemas de información, tanto primarios como de soporte.

Una vez que se han identificado los activos de la organización, el siguiente paso es valorarlos. Algunos activos se pueden valorar mediante una asignación monetaria (cuánto dinero perderíamos si este activo dejara de funcionar), mientras que otros requerirán una valoración cualitativa (si este activo dejara de funcionar tendríamos pérdidas muy altas, bajas, medias,...). Los criterios utilizados como base para la asignación de un valor a cada activo deben ser escritos en términos inequívocos. Los posibles criterios utilizados para determinar el valor de un activo incluyen su coste original, el duplicado o el coste de reposición. También se pueden dar criterios abstractos como, por ejemplo, el valor de la reputación de la organización [31].

Es conveniente valorar los activos en tres componentes (aunque algunas metodologías pueden considerar alguna más [37]) que son:

- Disponibilidad: Pérdidas potenciales en caso de que los procesos y servicios del sistema de información no estuvieran disponibles.

- Confidencialidad: Pérdidas potenciales en caso de que los datos del sistema de información fueran vulnerados.

- Integridad: Pérdidas potenciales en caso de que los datos, procesos y servicios del sistema de información tuvieran desperfectos.

La norma no especifica cómo se deben agregar estas tres componentes de valor. De hecho, indica varias posibilidades, como que el valor del activo debe ser el máximo de los valores de las tres componentes o la suma de ellas. Esta decisión corresponde a la organización. 
Al final, todos los activos deben valorarse en una base única. Es decir, los responsables de seguridad de la información, junto con los analistas encargados deben establecer una escala de niveles para valorar todos los activos. El número de niveles de la escala debe ser definido por los integrantes del modelo organizativo del SGSI. Una escala muy fina tiene la ventaja de que aporta mucha información, pero puede hacer poco manejable el SGSI. Lo normal es dar escalas de nivel de entre 5 y 10 valores.

Como indicamos anteriormente, los activos de soporte heredan su valor de los activos primarios a través de la influencia que tengan sobre los mismos. Es necesario entonces determinar las relaciones de dependencia de los activos primarios con respecto a los activos de soporte, y también las relaciones de dependencia que se den entre estos últimos.

\section{Identificación de las amenazas}

Una amenaza es una causa potencial de un incidente no deseado sobre los activos de la organización. Pueden ser de origen natural o humano, y podría ser accidental o deliberada. Algunas amenazas pueden afectar a más de un activo. En tales casos, pueden causar impactos diferentes dependiendo de qué activos se vean afectados [31].

En el Anexo $C$ de la norma ISO/IEC 27005 se puede consultar una lista detallada de amenazas.

\section{Identificación y valoración de las vulnerabilidades.}

Las vulnerabilidades son las debilidades de un activo o grupo de activos que pueden ser explotadas por una o más amenazas [31].

Las vulnerabilidades de los activos sobre cada amenaza tienen dos componentes: la probabilidad de materialización de la amenaza y la degradación causada por ésta sobre el valor del activo [37].

El Comité de Seguridad del SGSI no sólo debe considerar las distintas amenazas que se dan sobre los activos de su sistema de información, sino que debe determinar 
las probabilidades de materialización de las mismas, teniendo en cuenta que dichas probabilidades pueden cambiar a lo largo del tiempo. Estas probabilidades se suelen dar en términos de porcentaje de ocurrencia de las amenazas a partir de la frecuencia observada o estimada durante un periodo de tiempo [37]. También es posible dar escalas de valores similares a las indicadas anteriormente para la valoración de los activos.

En cuanto a la segunda componente de la vulnerabilidad, es necesario valorar la degradación causada sobre el valor de los activos, para de este modo calcular el impacto que podría tener la materialización de una amenaza. Por ejemplo, si la vulnerabilidad de un activo con respecto a una amenaza es muy alta, entonces la materialización de dicha amenaza tendrá consecuencias importantes sobre el valor del activo. La degradación también se suele dar en términos de porcentaje, aunque también es posible dar escalas de valores similares a las indicadas anteriormente para la valoración de los activos.

Como veremos, algunas metodologías como la española MAGERIT [37] eliminan el concepto de vulnerabilidad y directamente asignan a las amenazas las dos componentes de degradación y probabilidad de ocurrencia.

\section{Identificación de las consecuencias y valoración del impacto y el riesgo}

Una vez que se han valorado los activos, las amenazas y las vulnerabilidades, es necesario identificar cualitativamente las consecuencias y establecer indicadores de impaco y riesgo.

El impacto de una amenaza sobre un activo es una función del valor del activo y de la degradación de la vulnerabilidad para esa amenaza. El riesgo es una función del impacto y de la probabilidad de materialización de la amenaza.

La mayoría de las metodologías utilizan tablas de doble entrada para determinar estos indicadores según las dos variables de las que dependen, sin establecer modelos matemáticos, sino únicamente el criterio de los decisores que gestionan el sistema de información. La Tabla 5.5.1 muestra un ejemplo de impacto en función de sus variables valor del activo y degradación en una escala de cinco niveles. Ejemplos 
similares se pueden dar para el riesgo en las variables impacto y probabilidad.

Tabla 5.5.1: Tabla de Impacto

\begin{tabular}{ccccccc} 
& \multicolumn{7}{c}{ Valor del } & Activo & & & \\
\cline { 2 - 6 } Degrad. & & Muy Bajo & Bajo & Medio & Alto & Muy Alto \\
\cline { 2 - 6 } & Muy Bajo & Muy Bajo & Bajo & Medio & Alto & Muy Alto \\
\cline { 2 - 6 } & Bajo & Bajo & Medio & Alto & Muy Alto & Muy Alto \\
\hline Medio & Medio & Alto & Muy Alto & Muy Alto & Muy Alto \\
\hline Alto & Alto & Muy Alto & Muy Alto & Muy Alto & Muy Alto \\
\hline Muy Alto & Muy Alto & Muy Alto & Muy Alto & Muy Alto & Muy Alto \\
\hline
\end{tabular}

\subsubsection{Tratamiento de los riesgos}

Ante un análisis de los riesgos aceptado por los miembros del modelo organizativo del SGSI, el comité de seguridad debe tomar en cada caso una decisión de entre las cuatro opciones dadas en la Figura 5.5.3, de modo que es posible aceptar el riesgo (no hacer nada), evitarlo (si tiene la posibilidad de eliminar los activos implicados), transferirlo ${ }^{1}$ (a compañías aseguradoras) o reducirlo.

Esta última opción implica la implementación de controles o salvaguardas que reduzcan la vulnerabilidad de los activos ante las amenazas que se ciernen sobre ellos. Para reducir esta vulnerabilidad es necesario reducir sus componentes de degradación y probabilidad. Esto da lugar a dos tipos de controles [37]:

- Controles Preventivos: Reducen la probabilidad de materialización de las amenazas sobre los activos.

- Controles Paliativos: Reducen la degradación provocada sobre el valor de los activos al materializarse la amenaza.

\footnotetext{
${ }^{1}$ Hay que tener en cuenta que el hecho de transferir los riesgos a una compañía aseguradora no exime de responsabilidad a la organización.
} 


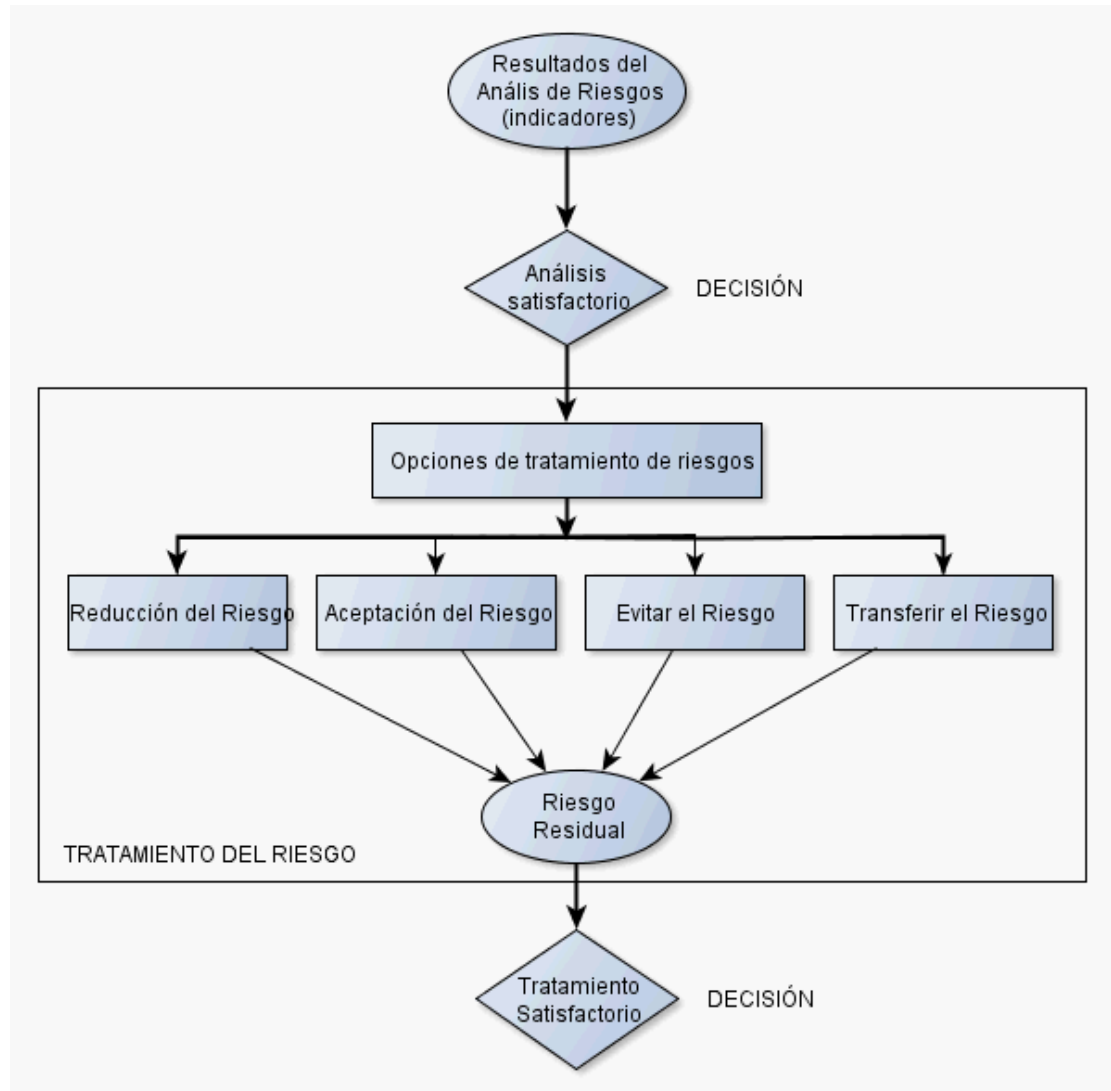

Figura 5.5.3: Tratamiento de Riesgos. ISO/IEC 27005 
CAPÍTULO 5 


\section{Capítulo 6 ANÁLISIS Y GESTIÓN BORROSA DE RIESGOS EN LOS SISTEMAS DE LA INFORMACIÓN}

En los Sistemas de la Información (SI), los activos están interrelacionados de modo que un fallo en uno de ellos se puede propagar por toda la red hasta llegar a afectar a los activos más valiosos del sistema, que suelen ser datos y servicios de las organizaciones. De hecho, el interés que tiene la mayoría de los activos del SI para la organización es el de garantizar el correcto funcionamiento de los servicios que ofrece, o la disponibilidad y confidencialidad de los datos que maneja. Así, el valor de dichos activos se hereda de estos últimos.

Cuando una amenaza se cierne sobre alguno de los activos del SI el riesgo se puede medir como función del valor del activo, de la probabilidad de materialización de la amenaza y de la degradación que ésta pueda causar sobre el valor del activo. Sin embargo, los expertos encargados de asignar tales valores a menudo aportan únicamente información vaga e imprecisa, de modo que las técnicas borrosas pueden ser muy útiles en este ámbito. En este capítulo proponemos un tratamiento borroso para los modelos de análisis y gestión de riesgos promovidos por las metodologías internacionales mediante el establecimiento de un modelo de representación y un modelo computacional adecuados. 


\subsection{Análisis y gestión de riesgos basado en la me- todología MAGERIT}

Tomando como base las normas de la serie 2700, los miembros de ISO/IEC han desarrollado y adaptado diferentes metodologías de análisis y gestión de riesgos en los SI. Algunos ejemplos de estas metodologías son MAGERIT, del Ministerio de Administraciones Públicas del Gobierno de España [37, 38, 39]; OCTAVE [3, 4]; desarrollada por la Universidad de Carnegie Mellon de Pensilvania (EEUU); MEHARI [41] del Club de la Securité de l'Information Francais; CRAMM [14] de la Agencia Estatal de Cómputo y Telecomunicaciones de Reino Unido; o la NIST SP 800-30 [45], del National Institute of Standard and Technology (EEUU).

Como hemos indicado en el glosario de ISO/IEC 270012009, los activos de un SI son los elementos del mismo que tienen valor para la organización (servidores, archivos, personal, instalaciones, hardware, software,...). Estos activos según algunas metodologías como MAGERIT [37, 38, 39] están interrelacionados, de modo que el valor total del SI se concentra en unos pocos activos, llamados activos terminales o primarios, y el resto adquieren valor en virtud de la relación que tengan con los activos terminales. Esta relación se define como la probabilidad de que un fallo provocado en un activo se transmita a los activos terminales [61, 64, 65, 69].

En general, diremos que el activo $A_{j}$ depende directamente del activo $A_{i}$, y lo denotaremos por $A_{i} \rightarrow A_{j}$, si un fallo en el activo $A_{i}$ provoca un fallo en el activo $A_{j}$ con cierta probabilidad. A dicha probabilidad la llamamos grado de dependencia directo de $A_{j}$ respecto a $A_{i}$, y la denotaremos por $d d\left(A_{i}, A_{j}\right)$.

Estas dependencias forman un grafo como el que se puede ver, a modo de ejemplo, en la Figura 6.1.1. En ella se observa que entre un activo cualquiera $A_{i}$ y un activo terminal $A_{k}$ (datos o productos y servicios) puede haber activos intermedios, a través de los cuales se puede propagar un fallo generado en $A_{i}$ hasta alcanzar el terminal $A_{k}$.

De este modo, estamos interesados en calcular la probabilidad de transmisión de un fallo entre $A_{i}$ y $A_{k}$. A esta probabilidad la llamaremos grado de dependencia indirecto entre $A_{i}$ y $A_{k}$ y la denotaremos por $D D\left(A_{i}, A_{k}\right)$. Estas probabilidades 


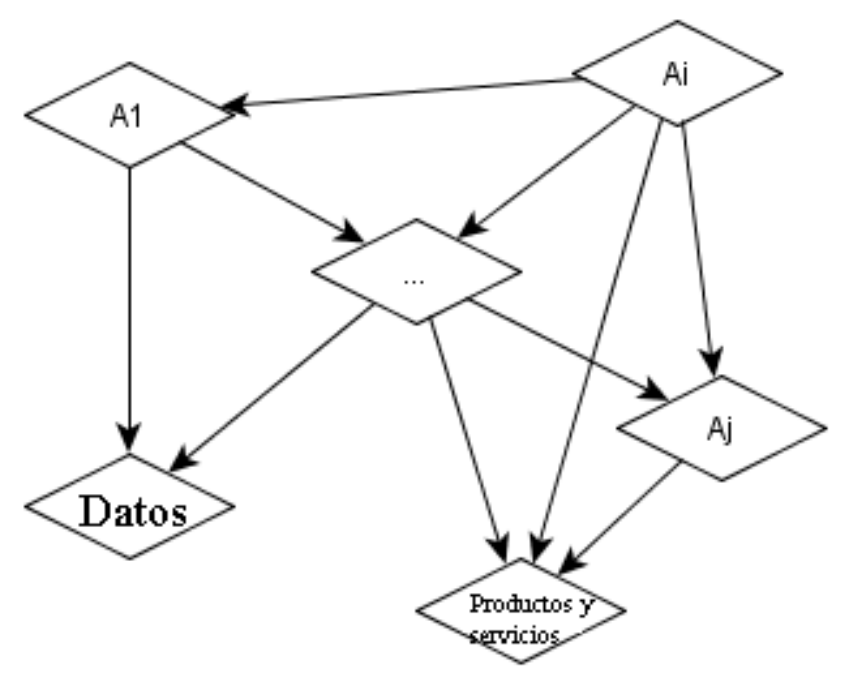

Figura 6.1.1: Esquema (o red) general de dependencias.

se pueden calcular en base a la Teoría del Cálculo de Probabilidades mediante la conocida fórmula de la probabilidad de la unión de varios sucesos independientes ${ }^{1}$ $[37,38,39]$. Dichos sucesos son la transmisión del fallo desde $A_{i}$ hasta $A_{k}$ a través de los distintos activos intermedios que determinan todos los posibles caminos en el grafo de dependencias con origen en $A_{i}$ y final en $A_{k}$.

Concretamente, $D D\left(A_{i}, A_{k}\right)$ se puede calcular mediante el siguiente algoritmo [61]: Denotemos por $\mathbf{P}=\left\{P_{1}, \ldots, P_{s}\right\}$ el conjunto de caminos que conectan $A_{i}$ con $A_{k}$.

A) Si todos los activos, salvo $A_{i}$ y $A_{k}$, en los caminos de $\mathbf{P}$ están influidos por un solo activo, entonces:

$$
D D\left(A_{i}, A_{k}\right)=\underset{j=1}{\stackrel{s}{+})} D D\left(A_{i}, A_{k} \mid P_{j}\right)
$$

donde $a(+) b=a+b-a b$ (en consistencia con la Teoría del Cálculo de Probabilidades),

$$
D D\left(A_{i}, A_{k} \mid P_{j}\right)=d d\left(A_{i}, A_{j 1}\right) d d\left(A_{j 1}, A_{j 2}\right) \ldots d d\left(A_{j n}, A_{k}\right)
$$

y $P_{j}$ es el camino

$$
P_{j}:\left(A_{i} \rightarrow A_{j 1} \rightarrow A_{j 2} \rightarrow \ldots \rightarrow A_{j n} \rightarrow A_{k}\right) .
$$

\footnotetext{
${ }^{1} \mathrm{Si} p_{1}=\operatorname{probabilidad}\left(A_{1}\right)$ y $p_{2}=\operatorname{probabilidad}\left(A_{2}\right)$ entonces $p=p_{1}+p_{2}-p_{1} p_{2}$ es la probabilidad de la unión de ambos sucesos si éstos son independientes entre sí.
} 
$B$ ) En otro caso, podemos asumir que los primeros $r$ caminos de $\mathbf{P}$ están formados por caminos en los que cada activo está a su vez influido por un único activo, y los restantes $s-r$ caminos incluyen activos que están a su vez influidos por varios activos simultáneamente. Entonces, para los $r$ primeros caminos procedemos como en $A$ ), y denotamos por $\mathbf{S}$ el conjunto de los $s-r$ caminos restantes. En $\mathbf{S}$ procedemos de la siguiente manera:

(i) Consideramos el conjunto de activos no terminales de $\mathbf{S}$ influidos por dos o más activos. Denotamos por $I$ a este conjunto. Sea $N I$ el subconjunto de $I$ de los activos que no están influidos por otro activo de $I$.

(ii) Consideremos un activo $A_{r}$ de $N I$. Entonces, simplificamos el camino de $\mathbf{S}$ que incluye el activos $A_{r}$ tomando $A_{i} \rightarrow A_{r} \rightarrow \ldots \rightarrow A_{k}$, con $d d\left(A_{i}, A_{r}\right)=$ $D D\left(A_{i}, A_{r}\right)$ que ha sido calculado en el paso anterior.

(iii) Eliminamos los caminos repetidos de $\mathbf{S}$ y mantenemos un único camino que agrupa a los anteriores.

(iv) Construimos de nuevo los conjuntos $I$ y $N I$ de $\mathbf{S}$.

(v) Si NI no es vacío volvemos al paso (ii). En otro caso, el algoritmo ha finalizado.

Denotemos el conjunto de caminos resultante por $\mathbf{S}=\left\{P_{1}^{\prime}, \ldots, P_{m}^{\prime}\right\}$ con $m \leq$ $s-r$. Entonces, el grado de dependencia de $A_{k}$, dado $A_{i}$ es

$$
\left.\left.D D\left(A_{i}, A_{k}\right)=\underset{j=1}{\stackrel{r}{+}}\right) D D\left(A_{i}, A_{k} \mid P_{j}\right) \underset{l=1}{+}\right) D D\left(A_{i}, A_{k} \mid P_{l}^{\prime}\right) .
$$

Como indicamos anteriormente, la razón de utilizar la operación $(+)$ es la consistencia con el Cálculo de Probabilidades. Veámoslo con un ejemplo:

Consideremos la estructura de dependencias de la Figura 6.1.2(a), y supongamos que un fallo en el activo $A_{1}$ puede provocar un fallo en el activo $A_{4}$ por la vía de $A_{2}$ o $A_{3}$ con las probabilidades indicadas. Como estas vías no son necesariamente excluyentes, del cálculo de probabilidades se sigue que

$$
p=p_{1} p_{3}+p_{2} p_{4}-p_{1} p_{3} p_{2} p_{4}=p_{1} p_{3}(+) p_{2} p_{4}
$$

Si el fallo se puede propagar a través de tres activos (Figura 5.1.2(b)) en lugar de 


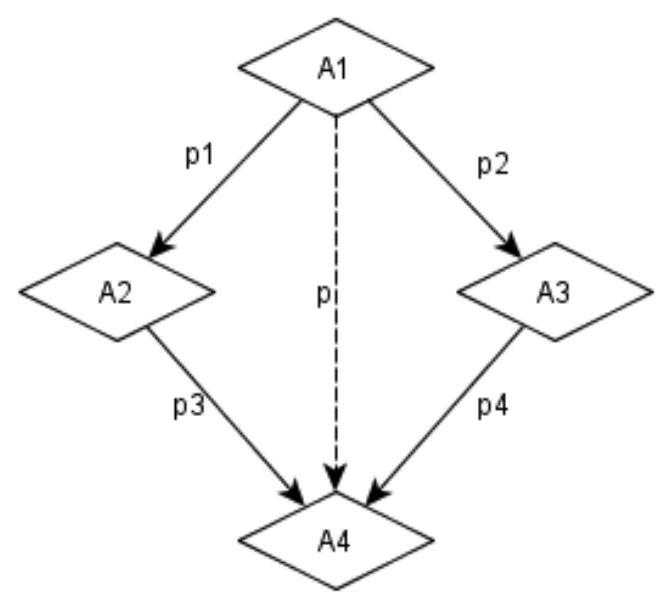

(a)

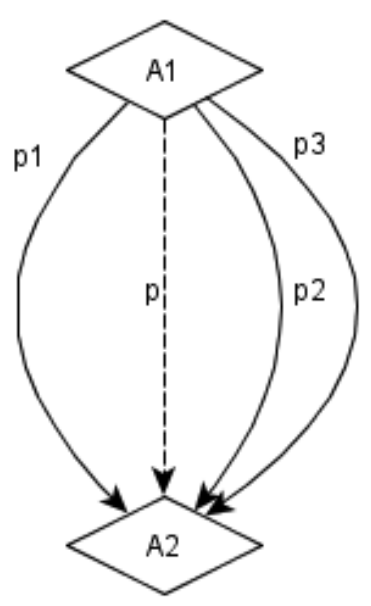

(b)

Figura 6.1.2: Estructuras de dependencias (ejemplos)

dos, entonces

$$
p=p_{1}+p_{2}+p_{3}-p_{1} p_{2}-p_{2} p_{3}-p_{1} p_{3}+p_{1} p_{2} p_{3}=p_{1}(+) p_{2}(+) p_{3}
$$

(por claridad, prescindimos de los activos intermedios).

En genaral, si la transmisión del fallo se puede realizar por $n$ vías, entonces, por inducción en $n$ se puede probar que

$$
p=p_{1}(+) p_{2}(+) \ldots(+) p_{n}=\sum_{i=1}^{n} p_{i}-\sum_{i<j} p_{i} p_{j}+\sum_{i<j<k} p_{i} p_{j} p_{k}+\ldots+(-1)^{n+1} \prod_{i=1}^{n} p_{i} .
$$

Una vez calculados los grados de dependencia indirectos entre todos los activos con respecto a los activos terminales, se puede calcular el valor acumulado $v_{j}$ de los activos de soporte a partir del valor propio $v_{k}$ de los activos terminales, que se suele dar mediante cinco componentes:

- Confidencialidad. ¿Qué daño causaría que lo conociera quien no debe? Esta valoración es típica de datos.

- Integridad. ¿Qué perjuicio causaría que estuviera dañado o corrupto? Esta valoración es típica de los datos, que pueden estar manipulados, ser total o parcialmente falsos o, incluso, faltar datos.

- Autenticidad. ¿Qué perjuicio causaría no saber exactamente quién hace o ha hecho cada cosa? Esta valoración es típica de servicios (autenticidad del 
usuario) y de los datos (autenticidad de quien accede a los datos para escribir o, simplemente, consultar).

- Trazabilidad. ¿Qué daño causaría no saber a quién se le presta tal servicio? O sea, ¿quién hace qué y cuándo? ¿qué daño causaría no saber quién accede a qué datos y qué hace con ellos?

- Disponibilidad. ¿Qué perjuicio causaría no tenerlo o no poder utilizarlo? Esta valoración es típica de los servicios.

Por tanto, $v_{k}$ y $v_{j}$ son vectores de cinco componentes, y escribiremos

$$
\vec{v}_{j}=\sum_{k} D D\left(A_{j}, A_{k}\right) \vec{v}_{k} .
$$

Una vez calculado el valor de todos los activos, consideraremos las amenazas que se ciernen sobre cada uno de ellos.

Las amenazas están caracterizadas por su probabilidad de materialización $f$ y por la degradación $\vec{d}=\left(d_{1}, d_{2}, d_{3}, d_{4}, d_{5}\right)$ que provocan en cada componente de valor de los activos. El impacto de una amenaza sobre un activo $A_{j}$ se mide por $\vec{I}_{j}=$ $\left(d_{1} v_{j 1}, d_{2} v_{j 2}, d_{3} v_{j 3}, d_{4} v_{j 4}, d_{5} v_{j 5}\right)$, y el riesgo sobre ese activo para la amenaza dada es $\vec{R}_{j}=f\left(d_{1} v_{j 1}, d_{2} v_{j 2}, d_{3} v_{j 3}, d_{4} v_{j 4}, d_{5} v_{j 5}\right)$.

Algunos modelos sugieren agregar las componentes de valor, impacto y riesgo mediante ponderaciones, de modo que, por ejemplo, si todas las componentes tienen el mismo peso, el impacto y el riesgo sobre un activo para una amenaza es proporcional al producto escalar del valor del activo por la degradación de la amenaza.

Para este modelo de análisis de riesgos basta entonces asignar los grados de dependencia directos (probabilidades) entre los activos del SI, el valor de los activos terminales, la degradación de las amenazas y la probabilidad de materialización de las mismas.

Estas valoraciones en general no serán sencillas, ya que en multitud de ocasiones no se cuenta con datos históricos y no hay posibilidad de realización de experimentos con objeto de estimar las probabilidades. Entonces, es fundamental recurrir al conocimiento experto para realizar estas valoraciones. Sin embargo, los expertos suelen 


\subsection{BORROSIFICACIÓN DEL ANÁLISIS DE RIESGOS BASADO EN LA} METODOLOGÍA MAGERIT

aportar información vaga e imprecisa y es aquí donde cobran sentido los planteamientos borrosos. La borrosificación del modelo descrito ha sido desarrollada en [61] y se expone a continuación.

\subsection{Borrosificación del análisis de riesgos basado en la metodología MAGERIT}

Consideremos en $[0,1]^{\mathcal{T F G}}$ la siguiente aritmética propuesta por Xu et al. [78]:

Si $\widetilde{A}_{1}=\left(a_{1}, b_{1}, c_{1}, d_{1} ; w_{\widetilde{A}_{1}}\right), \widetilde{A}_{2}=\left(a_{2}, b_{2}, c_{2}, d_{2} ; w_{\widetilde{A}_{2}}\right) \in[0,1]^{\mathcal{T} \mathcal{F} \mathcal{G}}$ entonces:

$\widetilde{A}_{1} \oplus \widetilde{A}_{2}=\left(a_{1}+a_{2}-a_{1} a_{2}, b_{1}+b_{2}-b_{1} b_{2}, c_{1}+c_{2}-c_{1} c_{2}, d_{1}+d_{2}-d_{1} d_{2} ; \min \left\{w_{\widetilde{A}_{1}}, w_{\widetilde{A}_{2}}\right\}\right)$

$\widetilde{A}_{1} \otimes \widetilde{A}_{2}=\left(a_{1} a_{2}, b_{1} b_{2}, c_{1} c_{2}, d_{1} d_{2} ; \min \left\{w_{\widetilde{A}_{1}}, w_{\widetilde{A}_{2}}\right\}\right)$

Veamos que ambas operaciones $(\oplus \mathrm{y} \otimes)$ están bien definidas:

Proposición 1. $\oplus \mathrm{y} \otimes$ son dos leyes de composición interna en el conjunto $[0,1]^{\mathcal{T} \mathcal{F}}$ que verifican las siguientes propiedades:

a) Ambas son conmutativas.

b) Ambas tienen elemento neutro.

c) Ambas son asociativas.

Demostración. Las propiedades a) y c) son triviales, ya que se verifican en cada componente. El elemento neutro de $\oplus$ es trivialmente el valor nítido $(0,0,0,0 ; 1)$, mientras que el elemento neutro de $\otimes$ es $(1,1,1,1 ; 1)$.

Veamos que son dos leyes de composición interna. Es decir, que si $\widetilde{A}_{1}$ y $\widetilde{A}_{2} \in[0,1]^{\mathcal{T F G}}$ entonces $\widetilde{A}_{1} \oplus \widetilde{A}_{2}, \widetilde{A}_{1} \otimes \widetilde{A}_{2} \in[0,1]^{\mathcal{T F}}$ :

Si $\widetilde{A}_{1}=\left(a_{1}, b_{1}, c_{1}, d_{1} ; w_{\widetilde{A}_{1}}\right), \widetilde{A}_{2}=\left(a_{2}, b_{2}, c_{2}, d_{2} ; w_{\widetilde{A}_{2}}\right) \in[0,1]^{\mathcal{T F} \mathcal{G}} \Rightarrow 0 \leq a_{1} \leq b_{1} \leq c_{1} \leq$ $d_{1} \leq 1,0 \leq a_{2} \leq b_{2} \leq c_{2} \leq d_{2} \leq 1$.

Entonces,

$0 \leq a_{1}+a_{2}-a_{1} a_{2}=1-\left(1-a_{1}\right)\left(1-a_{2}\right) \leq 1-\left(1-b_{1}\right)\left(1-b_{2}\right)=b_{1}+b_{2}-b_{1} b_{2} \leq 1$, y por la misma razón

$$
0 \leq b_{1}+b_{2}-b_{1} b_{2} \leq c_{1}+c_{2}-c_{1} c_{2} \leq d_{1}+d_{2}-d_{1} d_{2} \leq 1
$$


Además, $0 \leq \min \left\{w_{\widetilde{A}_{1}}, w_{\widetilde{A}_{2}}\right\} \leq 1$. Por tanto, $\widetilde{A}_{1} \oplus \widetilde{A}_{2} \in[0,1]^{\mathcal{T F \mathcal { G }}}$

Por otro lado, si $0 \leq a_{1} \leq b_{1} \leq 1$ y $0 \leq a_{2} \leq b_{2} \leq 1 \Rightarrow 0 \leq a_{1} a_{2} \leq b_{1} b_{2} \leq 1$, y por la misma razón $0 \leq b_{1} b_{2} \leq c_{1} c_{2} \leq d_{1} d_{2} \leq 1$. Por tanto, $\widetilde{A}_{1} \otimes \widetilde{A}_{2} \in[0,1]^{\mathcal{T F G}}$.

El interés de las operaciones dadas radica en la acotación del conjunto de números borrosos. Podemos asegurar que las operaciones entre términos lingüísticos borrosos trapezoidales de una escala en $[0,1]$ van a permanecer en $[0,1]^{\mathcal{T} \mathcal{F G}}$, y mediante una función de similitud los resultados de estas operaciones se podrán traducir en uno de los términos lingüísticos de la escala. Además, la operación $\oplus$ es consistente con la metodología establecida en la sección anterior, y permite interpretaciones en términos probabilísticos. Veamos estas cuestiones con más detalle.

La operación $(+)$ es un caso particular de $\oplus$, ya que un número real $a \in[0,1]$ se puede escribir en modo borroso como $a \approx \widetilde{a}=(a, a, a, a) \mathrm{y}$, por tanto,

$$
\begin{aligned}
\widetilde{a} \oplus \widetilde{b}=(a, a, a, a) \oplus(b, b, b, b) & =(a+b-a b, a+b-a b, \\
a+b-a b, a+b-a b) & =\widetilde{a(+) b} \approx a(+) b .
\end{aligned}
$$

Por otro lado, la operación $\otimes$ extiende de manera natural al producto de números reales.

Por tanto, con la definición de las operaciones $\oplus \mathrm{y} \otimes$ hemos conseguido extender las operaciones básicas que utiliza la metodología expuesta en la sección anterior al contexto de los números borrosos trapezoidales. Además, estas operaciones son consistentes con el Cálculo de Probabilidades.

Dadas las operaciones $\oplus \mathrm{y} \otimes$, la metodología para derivar la dependencia de cada activo de los activos terminales consiste en los siguientes pasos:

- Paso 1: Se establece una escala de términos lingüísticos borrosos (un modelo de representación).

- Paso 2: Se determina el grado de influencia de cada dos activos consecutivos en la estructura general de dependencias, estimando un término lingüístico de la escala dada. 
- Paso 3: Se determina el grado de influencia indirecto de los activos respecto de los activos terminales, mediante el algoritmo de la Sección 6.1 (borrosificando las operaciones). Este grado de dependencia será un número borroso trapezoidal.

Ejemplo 6.1 Consideremos la estructura de dependencias dada en la Figura 6.2.1 en la que el único activo terminal es $A_{6} \mathrm{y}$, por tanto, todo el valor del sistema se concentra en este activo.

Una escala lingüística útil para este proceso se muestra en la Tabla 6.2.1 y en la Figura 6.2.2.

En la Figura 6.2.1 se han señalado los grados de dependencia directos entre los activos, utilizando los términos lingüísticos de la Tabla 6.2.1.

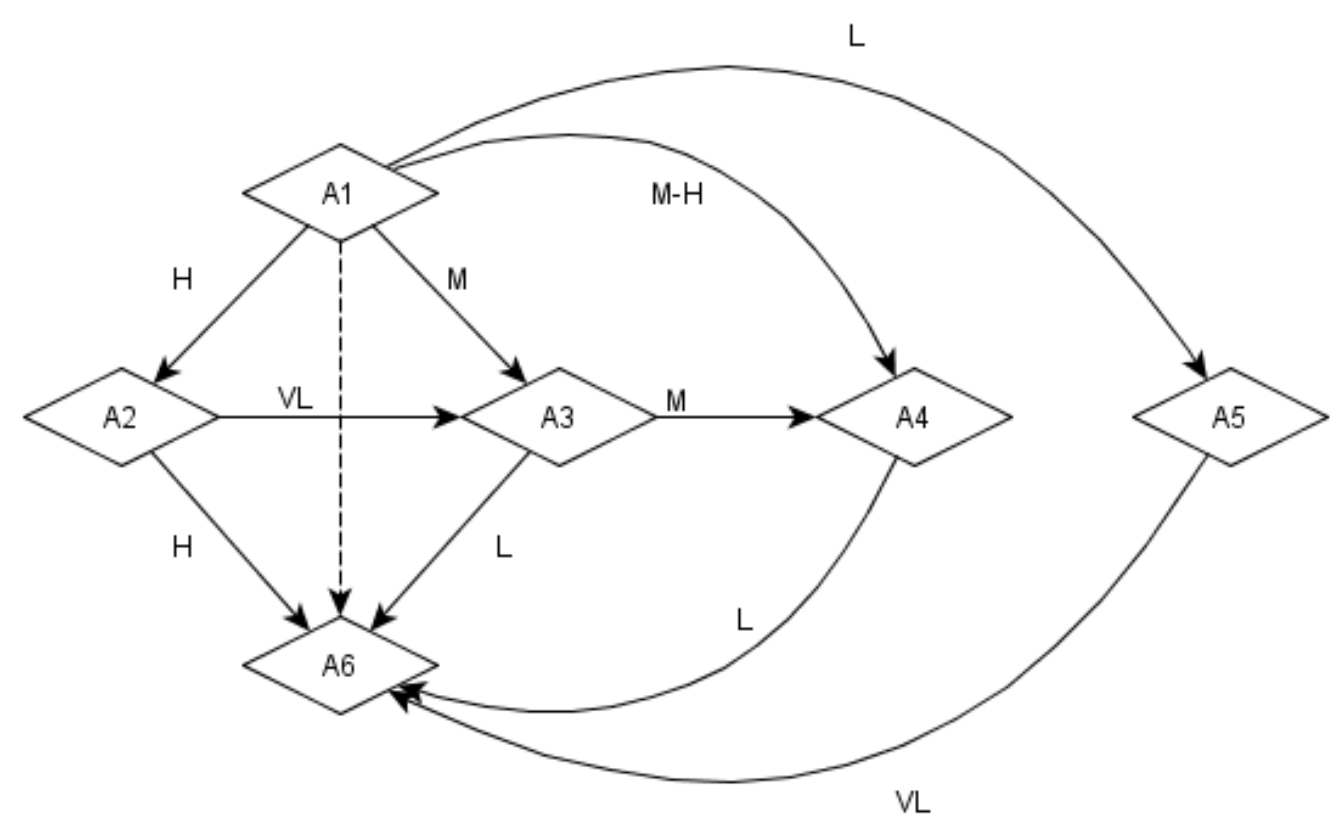

Figura 6.2.1: Estructura general de dependencias.

A continuación, calculamos el grado de influencia indirecto del activo $A_{1}$ sobre el activo $A_{6}$.

El conjunto de caminos que conectan $A_{1}$ con $A_{6}$ es: 


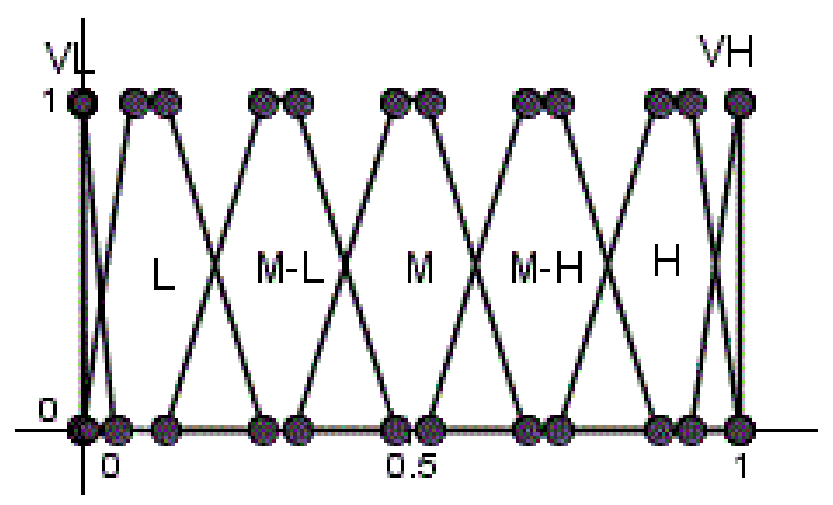

Figura 6.2.2: Expresión gráfica de la escala de la Tabla 6.2.1

Tabla 6.2.1: Términos lingüísticos

\begin{tabular}{cc}
\hline Término & Número borroso \\
\hline \hline Muy Bajo (VL) & $(0,0,0,0.05)$ \\
\hline Bajo (L) & $(0,0.075,0.125,0.275)$ \\
\hline Medio-Bajo (M-L) & $(0.125,0.275,0.325,0.475)$ \\
\hline Medio (M) & $(0.325,0.475,0.525,0.675)$ \\
\hline Medio- Alto (M-H) & $(0.525,0.675,0.725,0.875)$ \\
\hline Alto (H) & $(0.725,0.875,0.925,1)$ \\
\hline Muy Alto (VH) & $(0.925,1,1,1)$ \\
\hline
\end{tabular}

$$
\begin{aligned}
\mathbf{P}= & \left\{P_{1}:\left(A_{1} \rightarrow A_{2} \rightarrow A_{6}\right), P_{2}:\left(A_{1} \rightarrow A_{2} \rightarrow A_{3} \rightarrow A_{6}\right),\right. \\
& P_{3}:\left(A_{1} \rightarrow A_{2} \rightarrow A_{3} \rightarrow A_{4} \rightarrow A_{6}\right), P_{4}:\left(A_{1} \rightarrow A_{3} \rightarrow A_{6}\right), \\
& P_{5}:\left(A_{1} \rightarrow A_{3} \rightarrow A_{4} \rightarrow A_{6}\right), P_{6}:\left(A_{1} \rightarrow A_{4} \rightarrow A_{6}\right), \\
& \left.P_{7}:\left(A_{1} \rightarrow A_{5} \rightarrow A_{6}\right)\right\} .
\end{aligned}
$$

El activo $A_{3}$ está influido por $A_{1}$ y $A_{2}$, mientras que $A_{4}$ está influido por $A_{1}$ y $A_{3}$. Por tanto, aplicamos el apartado $B$ ) del algoritmo de la Sección 6.1, con $r=2$ y $\mathbf{S}=\left\{P_{2}, P_{3}, P_{4}, P_{5}, P_{6}\right\}$ y procedemos como sigue:

(i) $I=\left\{A_{3}, A_{4}\right\}$ y $N I=\left\{A_{3}\right\}$.

(ii) Seleccionamos $A_{3}$. Podemos simplificar $P_{2}, P_{3}, P_{4}$ y $P_{5}$ mediante $P_{2}^{\prime}:\left(A_{1} \rightarrow\right.$ $\left.A_{3} \rightarrow A_{6}\right), P_{3}^{\prime}:\left(A_{1} \rightarrow A_{3} \rightarrow A_{4} \rightarrow A_{6}\right), P_{4}^{\prime}:\left(A_{1} \rightarrow A_{3} \rightarrow A_{6}\right)$ y $P_{5}^{\prime}:$ $\left(A_{1} \rightarrow A_{3} \rightarrow A_{4} \rightarrow A_{6}\right)$, respectivamente, con $\left.\left.d \widetilde{\left(A_{1}, A_{3}\right.}\right)=\widetilde{D D\left(A_{1}, A_{3}\right.}\right)=$ $\left.\left(d d\left(\widetilde{A_{1}, A_{2}}\right) \otimes d d \widetilde{\left(A_{2}, A_{3}\right.}\right)\right) \oplus d \widetilde{\left(\widetilde{A_{1}, A_{3}}\right)}$.

(iii) $\mathbf{S}=\left\{P_{2}^{\prime}, P_{3}^{\prime}, P_{6}\right\}$ ya que $P_{2}^{\prime}=P_{4}^{\prime}$ y $P_{3}^{\prime}=P_{5}^{\prime}$.

(iv) $I=\left\{A_{4}\right\}$ y $N I=\left\{A_{4}\right\}$. 


\subsection{BORROSIFICACIÓN DEL ANÁLISIS DE RIESGOS BASADO EN LA METODOLOGÍA MAGERIT}

(v) Ir al paso (ii).

(ii) Seleccionamos $A_{4}$. Simplificamos los caminos $P_{3}^{\prime}$ y $P_{6}$ como $P_{3}^{\prime \prime}:\left(A_{1} \rightarrow A_{4} \rightarrow\right.$ $\left.A_{6}\right)$, y $P_{6}^{\prime}:\left(A_{1} \rightarrow A_{4} \rightarrow A_{6}\right)$, respectivamente, con $\left.d \widetilde{\left(A_{1}, A_{4}\right)}=\widetilde{D D\left(A_{1}\right.}, A_{4}\right)=$ $\left.\left(d d \widetilde{\left(A_{1}, A_{3}\right)} \otimes d d \widetilde{\left(A_{3}, A_{4}\right)}\right) \oplus d \widetilde{\left(A_{1}, A_{4}\right.}\right)$.

(iii) $\mathbf{S}=\left\{P_{2}^{\prime}, P_{3}^{\prime \prime}\right\}$ ya que $P_{3}^{\prime \prime} \equiv P_{6}^{\prime}$.

(iv) $I=\emptyset$ y $N I=\emptyset$.

(v) El algoritmo finaliza, ya que $N I=\emptyset$.

Finalmente, $\mathbf{S}=\left\{P_{2}^{\prime}, P_{3}^{\prime \prime}\right\}$ y el grado de dependencia de $A_{6}$ con respecto a $A_{1}$ es $\left.D \widetilde{\left(A_{1}, A_{6}\right.}\right)=D D\left(\widetilde{A_{1}, A_{6}} \mid P_{1}\right) \oplus D D\left(\widetilde{A_{1}, A_{6}} \mid P_{7}\right) \oplus D D\left(\widetilde{A_{1}, A_{6}} \mid P_{2}^{\prime}\right) \oplus D D\left(\widetilde{A_{1}, A_{6}} \mid P_{3}^{\prime \prime}\right)=$ $\left.\left.\left(d d \widetilde{\left(A_{1}, A_{2}\right)} \otimes d d \widetilde{\left(A_{2}, A_{6}\right)}\right) \oplus\left(d d \widetilde{\left(A_{1}, A_{5}\right.}\right) \otimes d d \widetilde{\left(A_{5}, A_{6}\right)}\right) \oplus\left(d d \widetilde{\left(A_{1}, A_{3}\right.}\right) \otimes d d \widetilde{\left(A_{3}, A_{6}\right)}\right) \oplus$ $\left(d d \widetilde{\left(A_{1}, A_{4}\right)} \otimes d \widetilde{\left(A_{4}, A_{6}\right)}\right)$

Sustituyendo los arcos por los términos lingüísticos que indican los grados de dependencia obtenemos:

$$
\begin{gathered}
{[[H \otimes[V L \otimes[(M \otimes L) \oplus L] \oplus H]] \oplus(L \otimes V L) \oplus} \\
\oplus\{[(H \otimes V L) \oplus M] \otimes[(M \otimes L) \oplus L]\} \oplus \\
\oplus\{[[[(H \otimes V L) \oplus M] \otimes M] \oplus M-H] \otimes L\}= \\
{[[(0.725,0.875,0.925,1) \otimes[(0,0,0,0.05) \otimes} \\
\otimes[((0.325,0.475,0.525,0.675) \otimes \\
\otimes(0,0.075,0.125,0.275)) \oplus \\
\oplus(0,0.075,0.125,0.275)] \oplus \\
\oplus(0.725,0.875,0.925,1)]] \oplus \\
\oplus((0,0.075,0.125,0.275) \otimes(0,0,0,0.05)) \oplus \\
\oplus\{[(0.725,0.875,0.925,1) \otimes(0,0,0,0.05)) \\
\oplus(0.325,0.475,0.525,0.675)] \otimes \\
\otimes[((0.325,0.475,0.525,0.675) \otimes
\end{gathered}
$$




$$
\begin{gathered}
\otimes(0,0.075,0.125,0.275)) \oplus \\
\oplus(0,0.075,0.125,0.275)]\} \oplus \\
\oplus\{[[((0.725,0.875,0.925,1) \otimes(0,0,0,0.05)) \oplus \\
\oplus(0.325,0.475,0.525,0.675)] \otimes \\
\otimes(0.325,0.475,0.525,0.675)] \oplus \\
\oplus(0.525,0.675,0.725,0.875)] \otimes \\
\otimes(0,0.075,0.125,0.275)\}= \\
(0.525,0.765,0.855,1) \oplus \\
\oplus(0,0,0,0.013) \oplus \\
\oplus(0.325,0.531,0.611,0.817) \oplus \\
\oplus(0,0.056,0.1,0.256)= \\
(0.679,0.891,0.949,1)
\end{gathered}
$$

El resto de grados de dependencia entre activos de soporte y activos terminales se calcula de forma análoga y los resultados se muestran en la Tabla 6.2.2.

Tabla 6.2.2: Grados de dependencia sobre el activo terminal

\begin{tabular}{cc}
\hline$A_{i}$ & $D \widetilde{D\left(A_{i}, A_{6}\right)}$ \\
\hline \hline$A_{1}$ & $(0.679,0.891,0.949,1)$ \\
\hline$A_{2}$ & $(0.725,0.875,0.925,1)$ \\
\hline$A_{3}$ & $(0,0.107,0.182,0.409)$ \\
\hline$A_{4}$ & $(0,0.075,0.125,0.275)$ \\
\hline$A_{5}$ & $(0,0,0,0.05)$ \\
\hline
\end{tabular}

\section{Valor acumulado en los activos de soporte}

Podemos escribir el valor propio en los activos terminales como un vector de cinco componentes $\widetilde{v}_{j}=\left(\widetilde{v}_{j_{(1)}}, \widetilde{v}_{j_{(2)}}, \widetilde{v}_{j_{(3)}}, \widetilde{v}_{j_{(4)}}, \widetilde{v}_{j_{(5)}}\right)$, donde $\widetilde{v}_{j_{(i)}}$ será un término lingüístico borroso asignado por un experto en la componente de valor $i$-ésima para el activo $A_{j}$.

El valor acumulado de un activo $A_{i}$ respecto de los activos terminales $A_{j}$ es

$$
\widetilde{v}_{i_{(l)}}=\sum_{j=1}^{n}\left(\widetilde{D\left(A_{i}, A_{j}\right) \otimes} \widetilde{v}_{j_{(l)}}\right) .
$$




\subsection{BORROSIFICACIÓN DEL ANÁLISIS DE RIESGOS BASADO EN LA METODOLOGÍA MAGERIT}

Para obtener el valor acumulado de los activos no terminales seguiremos, entonces, los siguientes pasos:

- Paso 4: Se estima el valor en cada componente de los activos terminales asignando un término lingüístico.

- Paso 5: Se calcula el valor acumulado en el resto de activos mediante la ecuación 6.2.1.

Ejemplo 6.1 (continuación) Supongamos que los expertos asignan un valor sobre $A_{6}$ dado por sus cinco componentes de $\widetilde{v}_{6}$ :

- Confidencialidad $v_{6(1)}=[10,20] \times 10^{6}$ unidades monetarias.

- Integridad $v_{6(2)}=[5,8] \times 10^{6}$ unidades monetarias.

- Autenticidad $v_{6(3)}=[12,15] \times 10^{6}$ unidades monetarias.

- Trazabilidad $v_{6(4)}=[1,2] \times 10^{6}$ unidades monetarias.

- Disponibilidad $v_{6(5)}=[15,20] \times 10^{6}$ unidades monetarias.

Entonces, el valor acumulado sobre $A_{1}$ se calcula aplicando la Ecuación 6.2.1, de donde obtenemos la Tabla 6.2.3

Tabla 6.2.3: Valor acumulado de $A_{1}$ en cada componente

\begin{tabular}{cc}
\hline Componente & $\widetilde{v}_{1_{(l)}}$ \\
\hline \hline Confidencialidad & $(6.79,8.91,18.98,20)$ \\
\hline Integridad & $(3.395,4.455,7.592,8)$ \\
\hline Autenticidad & $(8.148,10.692,14.235,15)$ \\
\hline Trazabilidad & $(0.679,0.891,1.898,2)$ \\
\hline Disponibilidad & $(10.185,13.365,18.98,20)$ \\
\hline
\end{tabular}

\section{Amenazas. Indicadores de impacto y riesgo, y uso de la función de similitud}

Una amenaza es un vector $\overrightarrow{\vec{u}}=(\widetilde{f}, \widetilde{D})$ cuyas componentes son la frecuencia y la degradación. Esta última, a su vez se puede dar en cada componente de valor.

Consideremos una amenaza sobre el activo $A_{j}$ cuya degradación en cada componente viene dada por el vector $\overrightarrow{\widetilde{D}}=\left(\widetilde{d}_{1}, \widetilde{d}_{2}, \widetilde{d}_{3}, \widetilde{d}_{4}, \widetilde{d}_{5}\right)$. Es decir, que la amenaza provoca una degradación de gravedad $\widetilde{d}_{i}$ en la componente $i$-ésima del activo. 
Cuando la amenaza se materializa, cada componente se verá afectada según la expresión

$$
\widetilde{I}_{j_{(i)}}=\widetilde{d}_{i} \otimes \widetilde{v}_{j_{(i)}}
$$

$\widetilde{I}_{j_{(i)}}$ es el impacto provocado sobre el activo atacado. Para calcular el riesgo sobre este activo podemos utilizar la expresión

$$
\widetilde{R}_{j_{(i)}}=\widetilde{I}_{j_{(i)}} \otimes \widetilde{f}
$$

Una vez calculado el impacto provocado por una amenaza materializada sobre un activo del sistema podemos calcular el impacto transmitido a los activos inferiores que dependen del activo atacado.

Si $A_{j}$ es el activo sobre el que se ha materializado la amenaza y $A_{k}$ un activo inferior cuyo grado de dependencia con $A_{j}$ es $\widetilde{D D\left(A_{j}, A_{k}\right)}$, entonces la amenaza sobre el activo

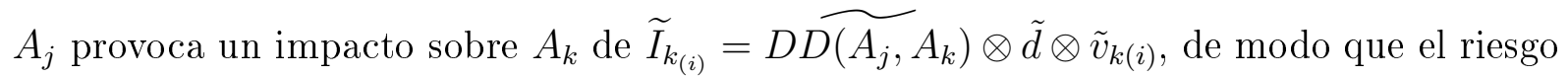
sobre el activo inferior será $\widetilde{R}_{k_{(i)}}=\widetilde{I}_{k_{(i)}} \otimes \tilde{f}$.

Por tanto, tras identificar las amenazas, su degradación y su frecuencia, los pasos que seguiremos para identificar el impacto y el riesgo sobre los activos atacados son:

- Paso 6: Se calculan los parámetros de impacto y riesgo en cada activo mediante las Ecuaciones 6.2.2 y 6.2.3.

- Paso 7: El resultado borroso trapezoidal se asocia a uno de los términos lingüísticos de la escala dada mediante una función de similitud.

Consideremos una amenaza sobre el activo $A_{1}$ con una degradación $\widetilde{D}=(H, L, M$, $V L, M)$ y una frecuencia $\tilde{f}=M$. Entonces, los indicadores de impacto y riesgo, que resultan de las Ecuaciones 6.2.2 y 6.2.3 se muestran en la Tabla 6.2.4

Tabla 6.2.4: Indicadores de impacto y riesgo sobre $A_{1}$

\begin{tabular}{ccc}
\hline Componente & Impacto & Riesgo \\
\hline \hline Confidencialidad & $(4.92,7.79,17.55,20)$ & $(1.59,3.70,9.21,13.5)$ \\
\hline Integridad & $(0,0.33,0.94,2.2$ & $(0,0.15,0.49,1,48$ \\
\hline Autenticidad & $(2.64,5.07,7.47,10.12)$ & $(0.86,2.41,3.92,6.83)$ \\
\hline Trazabilidad & $(0,0,0,0.01)$ & $(0,0,0,0.006)$ \\
\hline Disponibilidad & $(3.31,6.34,9.96,13.5)$ & $(1.07,3.01,5.23,9.11)$ \\
\hline
\end{tabular}




\section{Capítulo 7 SELECCIÓN DE SALVAGUARDAS MEDIANTE METAHEURÍSTICAS Y PROGRAMACIÓN DINÁMICA}

\subsection{Introducción}

Un problema de optimización [18] es un problema con una gran cantidad de soluciones posibles (tal vez infinitas soluciones, llamadas soluciones factibles) junto con una función de aptitud sobre dicho conjunto que nos indica cómo de buenas son esas soluciones, de tal forma que podemos comparar en todo momento la bondad de dos soluciones factibles diferentes.

Los problemas de optimización suelen plantearse mediante la maximización o minimización de una función (la función objetivo) con respecto a una serie de restricciones que definen el conjunto de soluciones factibles:

$$
\begin{aligned}
& \text { Minimizar } f(x) \\
& \text { s.a } \\
& g_{1}(x)=0 \\
& \ldots \\
& g_{p}(x)=0
\end{aligned}
$$

El ejemplo más sencillo posible consiste en minimizar o maximizar una función real de variable real, de modo que los métodos analíticos son suficientes en muchos casos.

Un método analítico bastante utilizado en algunos problemas de optimización con restricciones es el método de los multiplicadores de Lagrange, que consiste en definir la función lagrangiana de la función objetivo y los llamados multiplicadores de Lagrange 
$\lambda_{1}, \ldots, \lambda_{p}:$

$$
L\left(f, \lambda_{1}, \ldots, \lambda_{p}\right)=f(x)-\sum_{i=1}^{p} \lambda_{i} g_{i}(x) .
$$

Entonces, se eliminan los multiplicadores de Lagrange en el sistema de ecuaciones:

$$
\begin{gathered}
\nabla L\left(f, \lambda_{1}, \ldots, \lambda_{p}\right)=0 \\
g_{1}(x)=0 \\
\ldots \\
g_{p}(x)=0
\end{gathered}
$$

Este sistema de ecuaciones suele resolverse mediante algoritmos de Cálculo Numérico que llevan a soluciones exactas.

Otro conjunto de problemas de optimización más modernos pero ya clásicos son los problemas combinatorios. Un ejemplo paradigmático es el conocido problema del viajante de Comercio, que se puede formular de la siguiente manera: Un comercial debe recorrer $n$ ciudades comenzando su viaje en una de ellas, a la cual debe volver al finalizar la jornada. El comercial se pregunta cómo debe hacer el recorrido para minimizar los costes, es decir, cuál es el camino más corto que debe recorrer bajo la restricción de visitar todas las ciudades propuestas una única vez. Este problema de minimización tiene $(n-1)$ ! soluciones factibles, es decir, existen $(n-1)$ ! caminos que verifican la restricción planteada. Si tenemos cuatro ciudades, entonces existen 6 posibles caminos y el problema es trivial, pero si tenemos sólo 15 ciudades entonces tenemos más de un billón de soluciones factibles. Por tanto, este problema es analíticamente intratable ${ }^{1}$

Para resolver este problema hemos de implementar algoritmos computacionales (en ordenador) que busquen soluciones en el conjunto factible. Para ello, suele definirse una estructura de entorno de modo que la función de aptitud (que a menudo coincide con la función objetivo) pase de una solución a otra valorando la bondad de las soluciones e inclinándose hacia las zonas en que se encuentran las mejores.

Otros enfoques tratan de emular procesos naturales como el comportamiento de las colonias de hormigas, la leyes evolutivas o el enfriamiento y la adaptación de los átomos

\footnotetext{
${ }^{1}$ De hecho, en muchas ocasiones no encontraremos la mejor solución del problema sino que tendremos que conformarnos con una solución suficientemente buena. A este enfoque se le denomina enfoque satisfaciente, frente al enfoque optimizador que puede plantearse en otros problemas.
} 


\subsection{INTRODUCCIÓN}

de materiales metálicos a altas temperaturas.

La cardinalidad del espacio de soluciones factibles es clave en la historia de la matemática computacional hasta el punto de establecerse una clasificación de los problemas según el tiempo computacional de los algoritmos que pueden resolverlos.

Para entender este concepto podemos recurrir al siguiente ejemplo [47]: Consideremos un censo desordenado de $N$ ciudadanos y busquemos en este censo a una persona concreta por su nombre y apellidos. En el peor de los casos, para encontrar a esta persona hemos de leer todos los elementos del censo. Entonces, se dice que el tiempo computacional de este algoritmo de búsqueda es lineal para este problema (lo cual suele denotarse por $O(n)$ ) porque es proporcional al tamaño del problema (el factor de proporción dependería del tiempo que necesita la máquina para pasar de una instancia a otra en la base de datos).

Ahora supongamos que la lista está ordenada alfabéticamente. Entonces, podemos dividir el censo por la mitad, y ver si el apellido de la persona buscada está en la primera mitad o en la segunda, desechando la mitad en la que no está. Si repetimos sucesivamente el proceso, el tiempo que tardamos en el peor de los casos en encontrar a la persona es el tiempo que tardamos en quedarnos con una sola instancia (la última persona). Y puesto que en cada iteración la población se reduce a la mitad, terminaremos en la $n$-ésima iteración tal que $\frac{N}{2^{n}}=1$, por tanto $n=\log _{2}(N)$. De modo que el tiempo computacional es logarítmico, es decir proporcional a $\log _{2}(N)$, que suele denotarse por $O\left(\log _{2}(N)\right)$. El factor de proporcionalidad depende del tiempo que la máquina necesite para realizar una iteración.

Durante la década de 1970 se determinaron dos clases fundamentales de problemas. Los problemas que pueden ser resueltos por algún algoritmo en tiempo polinomial (de clase P) y los problemas de clase NP que no pueden ser resueltos por ningún algoritmo en tiempo polinomial pero que se puede saber en tiempo polinomial si un elemento dado es solución del problema. Por ejemplo, una ecuación algebraica de grado 5 se puede resolver mediante un algoritmo iterativo más o menos complejo, pero saber si un número real dado es solución de dicha ecuación es bastante sencillo.

Finalmente, los llamados problemas NP-duro son problemas para los cuales no existen algoritmos de resolución en tiempo polinomial y tampoco es posible comprobar en tiempo polinomial si un elemento es solución de dicho problema. Por ejemplo, el problema de 
viajante de comercio descrito anteriormente es un problema NP-duro, ya que para ver si un camino es solución de dicho problema habría que compararlo con el resto de caminos del grafo. Por la misma razón, la mayoría de problemas de optimización son problemas NP-duros.

En minería de datos, estadística multivariante o análisis de riesgos, dado que generalmente tratamos de estimar diferentes elementos algebraicos a partir de datos que a su vez pueden estar afectados por ruido, es bastante habitual utilizar algoritmos de búsqueda de soluciones que minimizan una función de coste definida ad hoc, lo cual, como hemos visto, suele ser un problema NP-duro.

Un método que podríamos calificar de tradicional, pero que nos va a permitir ilustrar las dificultades básicas que deben superar las técnicas metaheurísticas, es el Algoritmo de Descenso por el Gradiente. El método consiste en lo siguiente:

Supongamos que queremos minimizar la función $F\left(x_{1}, \ldots, x_{p}\right)$. Entonces la ecuación $z=F\left(x_{1}, \ldots, x_{p}\right)$ define una hipersuperficie de $\mathbb{R}^{p+1}$.

El gradiente de esta hipersuperficie se define como

$$
\nabla F(x)=\left(\frac{\partial F}{\partial x_{1}}, \ldots, \frac{\partial F}{\partial x_{p}}\right)(x)
$$

y verifica la interesante propiedad de que en cada punto $(x, z)$ de la hipersuperficie el vector $\nabla F(x)$ es la dirección de máxima ganancia de $F$, es decir, la dirección en que el valor de $F$ crece más rápidamente, y recíprocamente $-\nabla F(x)$ representa la dirección de máxima pérdida (aquélla en que el valor de $F$ se reduce más rápidamente). Finalmente, en las direcciones perpendiculares a $\nabla F(x)$ el valor de $F$ es constante. Suele decirse que $\nabla F(x)$ apunta a los picos de la hipersuperficie $z=F\left(x_{1}, \ldots, x_{p}\right)$, mientras que $-\nabla F(x)$ apunta a los valles.

Esta propiedad nos permitiría definir un algoritmo rápido de minimización de una función (y recíprocamente de maximización): Dada una solución factible $x=a$ del problema, hemos de seguir la dirección de $-\nabla F(a)$. Por tanto, $b=a-\lambda \nabla F(a)$ verifica que $F(b) \leq F(a)$, de modo que si $b$ es una solución factible del problema de minimización, entonces es mejor solución que $a$, y en la dirección de $b$ están las mejores soluciones que se pueden encontrar partiendo de $a$. El valor de $\lambda$ suele denominarse tasa de mejora.

La dificultad que presenta el método de descenso por el gradiente radica en que se 
queda fácilmente atrapado en mínimos locales, como se muestra en la Figura 7.1.1. Cuando el algoritmo es atraído por un mínimo local, ya no encuentra una solución mejor en la dirección contraria al gradiente y finaliza. La rapidez del algoritmo es tan alta que no explora zonas en las que puede haber valores menores que el mínimo encontrado.

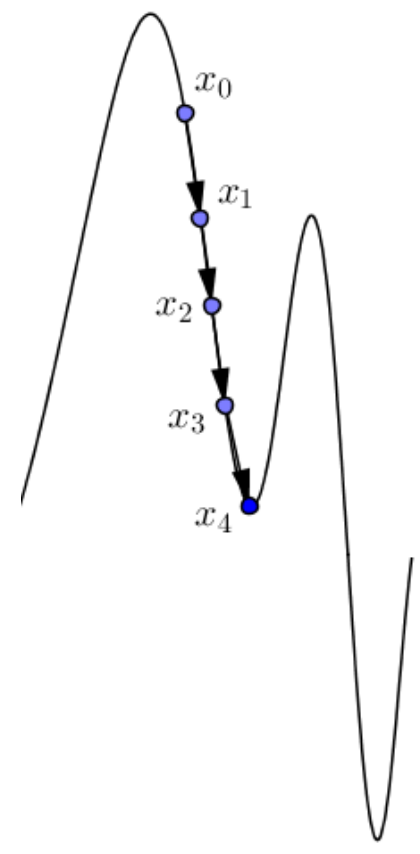

Figura 7.1.1: El problema de los mínimos locales

Otra cuestión a tener en cuenta es la tasa de mejora $\lambda$ que indica la longitud del paso (módulo del vector) de una solución a otra. Si este valor es muy pequeño se puede obtener una gran precisión (aunque no resuelve la cuestión de los mínimos locales) pero el algoritmo se ralentiza excesivamente. Por contra, si es demasiado grande, el algoritmo podría estar rebotando eternamente entre las laderas del valle, sin llegar a caer al mínimo local.

Cuando el algoritmo cae en mínimos locales suele emplearse la expresión «dar una patada al algoritmo» para superar el atasco. De hecho, el problema de los mínimos locales, así como el de la representación de las soluciones factibles del problema que a veces no puede darse en la forma adecuada para aplicar métodos analíticos, suelen resolverse introduciendo mecanismos que establezcan un buen compromiso entre la convergencia del algoritmo y la capacidad de explorar toda la región de soluciones factibles, enfoque que origina el establecimiento de las técnicas metaheurísticas modernas. 
El término «heurística» viene del griego heuriskein, eureka. El famoso «lo encontré» de la leyenda arquimediana se utiliza para sugerir un conjunto de técnicas que buscan una solución apropiada sobre un conjunto de soluciones más o menos grande. La limitación de las técnicas heurísticas, anteriores a las metaheurísticas, consiste precisamente en su incapacidad para remontar óptimos locales. Por su parte, la metaheurísticas son también técnicas de búsqueda de soluciones con mecanismos para escapar a dichos óptimos locales.

\subsubsection{Recocido simulado}

Como se ha indicado en el Capítulo 3, una de las técnicas metaheurísticas más utilizadas por su potencia y sencillez es el Recocido Simulado.

El Recocido (o enfriamiento) Simulado (Simulated Annealing -SA-) trata de reproducir el proceso termodinámico de recocido del acero. Este proceso consiste en calentar el material a altas temperaturas de modo que los átomos, que se encuentran en un nivel de energía determinado (en un mínimo local a temperatura ambiente) adquieran un nuevo nivel de energía que les permita remontar este mínimo local. Posteriormente, un proceso de enfriamiento suficientemente lento en un baño térmico llevará a las partículas a una configuración espacial cristalina con un nivel de energía menor al inicial, alcanzándose el mínimo global.

El esquema de la metaheurística, introducida por Kirkpatrick et al. [33] en 1983, es similar al proceso de recocido. La función a optimizar es la energía (o entropía) del sistema, y las distintas configuraciones de las partículas son las posibles soluciones del problema a optimizar. La temperatura será una función decreciente con el tiempo que nos ayudará a tomar decisiones sobre la aceptación de soluciones (configuraciones) vecinas. Cuando la temperatura es alta, aceptaremos prácticamente cualquier configuración por mala que sea, cuando es baja el criterio de aceptación será más restrictivo, pasaremos normalmente a soluciones mejores que la actual. Se demuestra que si el proceso de enfriamiento es suficientemente lento se converge al mínimo global. A este proceso de enfriamiento se le denomina forma de enfriamiento.

El pseudocódigo del recocido simulado para un problema de maximización es el siguiente:

- Generar una solución factible inicial $x_{0}$. Hacer $x^{*}=x_{0}, f^{*}=f\left(x_{0}\right), i=0$. Seleccionar 


\subsection{INTRODUCCIÓN}

un valor para la temperatura inicial $t_{0}=T\left(t_{i}\right.$ es la temperatura en el paso. $\left.i\right)$

- Repetir hasta satisfacer el criterio de parada:

- Generar aleatoriamente $y \in N\left(x_{i}\right)$ donde $x_{i}$ es la solución en el paso $i$.

- Si $f(y)-f\left(x_{i}\right) \geq 0$, entonces

$\circ x_{i+1}=y$.

○ Si $\left(f\left(x^{*}\right)>f\left(x_{i}\right)\right)$, entonces $x^{*}=x_{i}, f^{*}=f\left(x_{i}\right)$.

en otro caso:

$\circ p \sim U(0,1)$.

○ Si $p \leq e^{-\left(f(y)-f\left(x_{i}\right)\right) / t_{i}}$, entonces $x_{i+1}=y$.

- $i=i+1$.

- Actualizar temperatura.

Suele tomarse la expresión $e^{-\left(f(y)-f\left(x_{i}\right)\right) / T}$ en analogía con la distribución de MaxwellBoltzmann para los sistemas termodinámicos.

Como vemos, los elementos de recocido simulado son el espacio de búsqueda, la estructura de entorno $\left(N\left(x_{i}\right)\right)$, la solución inicial, la temperatura inicial $\left(t_{0}\right)$, el proceso (o forma) de enfriamiento y el criterio de parada. Estos elementos se detallan a continuación:

1. El espacio de búsqueda. Es el conjunto de todas las posibles soluciones a considerar. No necesariamente está restringido a las soluciones factibles puesto que en ocasiones puede ser conveniente saltar a una solución del entorno no factible, intentando evitar quedarnos atrapados en los óptimos locales [49].

2. El entorno de una solución estará determinado por la naturaleza y la topología de la región factible. Por ejemplo, si éste está formado por vectores binarios cuyas componentes denotan la selección o no de determinados elementos, la estructura de entorno vendrá definido por un número reducido de cambios en esas componentes, mientras que si el espacio de soluciones es continuo, entonces la estructura de entorno está determinado por las esferas de un cierto radio centradas en las soluciones factibles.

3. La solución inicial puede influir en la dirección que sigue el proceso y esto puede afectar a la convergencia. Por tanto, es necesario analizar el efecto de la solución inicial y considerar si fuera necesario la implementación de algoritmos específicos 
para la selección de una solución inicial adecuada. Sin embargo, es usual tomar la solución inicial de forma aleatoria por cuestiones de costes computacionales.

4. El valor inicial de la temperatura debe tomarse de forma que la probabilidad de aceptación de soluciones peores que la inicial sea alta $(>0.9)$, es decir, tal que $e^{-\left(f(y)-f\left(x_{0}\right)\right) / T} \geq 0.9$ si $y$ es una solución peor que la solución inicial [16].

5. Con respecto al proceso de enfriamiento, es decir, la actualización de la temperatura, existen dos enfoques diferentes. En el primero de ellos (el enfoque estático) los parámetros son fijos y no cambian a lo largo del enfriamiento. El esquema estático más simple fue propuesto por el propio Kirkpatrick [33] en su trabajo de 1983 y consiste en actualizar la temperatura mediante la expresión $T_{h L}=\alpha^{h} T_{0}$ con $0<\alpha<1$ constante. Es decir, se mantiene la temperatura durante $L$ iteraciones y a partir de ahí se reduce multiplicando por $\alpha$ durante otras $L$ iteraciones. Se ha probado empíricamente que valores altos de $\alpha$ implican una mejor ejecución del algoritmo y típicamente se utiliza $\alpha=0.95$.

En el segundo enfoque (dinámico) los parámetros son adaptativos, por ejemplo $T_{k+1}=\alpha\left(T_{k}\right) \times T_{k}$. De modo que $\alpha$ depende de $T_{k}$.

6. Finalmente, para el criterio de parada existen múltiples posibilidades, como que la solución no haya mejorado en el último $p \%$ de las iteraciones, o bien parar si el número de transiciones aceptadas es menor que el $p \%$ de $L$ tras $k$ iteraciones de $L$ pasos, etcétera.

\subsubsection{Programación dinámica}

Otro enfoque de optimización que utilizaremos en esta tesis es la programación dinámica [50], que se puede utilizar para resolver problemas de decisión secuenciales o polietápicos y, por tanto, es la técnica ideal cuando el sistema está representado por un grafo dirigido, como es nuestro caso. Estas decisiones están interrelacionadas entre sí, de forma que las decisiones que se tomen en cada etapa influyen sobre las decisiones tomadas sobre el resto de etapas.

Para describir este enfoque consideraremos el siguiente ejemplo. 


\subsection{INTRODUCCIÓN}

Ejemplo de la diligencia [50]: Consideremos un viajante que debe ir de la ciudad 1 a la ciudad 12 en el mapa de ciudades representado por el grafo de la Figura 7.1.2. Los nodos del grafo representan las ciudades y los arcos representan las distancias. Se trata de determinar la ruta óptima.

Se pueden seguir varias estrategias, por ejemplo, elegir en cada nodo la ciudad más cercana. Mediante esta estrategia el viajante realizaría la ruta

$$
R_{1}: 1 \rightarrow 3 \rightarrow 6 \rightarrow 9 \rightarrow 10 \rightarrow 12
$$

con una distancia total recorrida de 13, mientras que la ruta óptima:

$$
R_{2}: 1 \rightarrow 2 \rightarrow 6 \rightarrow 8 \rightarrow 11 \rightarrow 12
$$

tiene una distancia total recorrida de 8 .

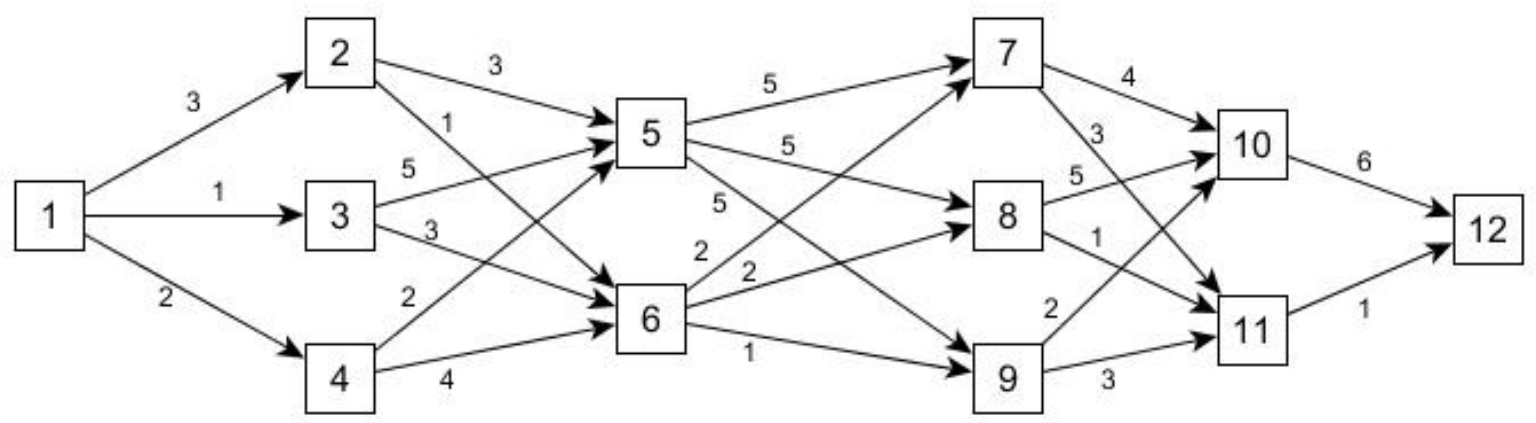

Figura 7.1.2: Mapa ejemplo de la diligencia

La cantidad de rutas posibles puede calcularse multiplicando el número de posibles decisiones en cada nodo: $n=3 \times 2 \times 3 \times 2=36$. Por tanto, la dificultad del problema crece rápidamente con la estructura de la red. Por ejemplo, para 20 etapas intermedias con 10 nodos en cada etapa, la cantidad de rutas posibles sería $10^{20}$.

Como vemos, los problemas de programación dinámica se caracterizan por la toma de decisiones polietápica, y en la etapa $n$-ésima tenemos los siguientes elementos:

- $e_{n}=$ estado de entrada,

- $x_{n}=$ decisión,

- $s_{n}=$ estado de salida, 
- $c_{n}\left(e_{n}, x_{n}\right)=$ coste de la decisión,

y el coste de una decisión polietápica completa será la acumalación de todos los costes de las decisiones de cada etapa.

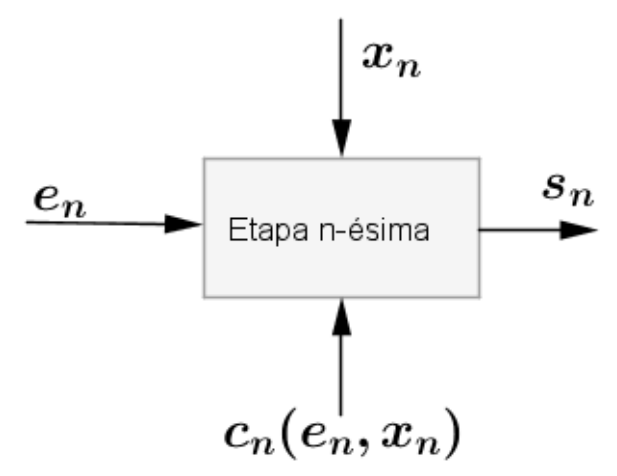

Figura 7.1.3: Elementos de una etapa de programación dinámica

La solución de un problema de programación dinámica se basa en el principio de optimalidad de Bellman [50]:

"Una política óptima tiene la propiedad de que conocidos el estado y la decisión iniciales, las restantes decisiones deben constituir una política óptima con respecto al estado resultante de la primera decisión."

Este principio nos lleva a dos estrategias conocidas como recursión hacia delante o recursión hacia atrás. En el ejemplo de la diligencia, se ha aplicado la recursión hacia atrás para obtener la decisión óptima. Este planteamiento consiste en lo siguiente:

Consideremos las etapas del problema de la diligencia que se han establecido en la Figura 7.1.4. Procedemos de la siguiente manera:

1. Si consideramos la Etapa 5, entonces estamos en la ciudad 10 o en la ciudad 11, y la decisión es trivial ya que sólo hay una alternativa. El coste de cada decisión puede verse en la Tabla 7.1.1 (Etapa 5).

2. Si consideramos la Etapa 4, entonces estaremos en las ciudades 7, 8 o 9, desde las que podemos ir a 10 u 11 con los costes acumulados de la Etapa 5 que se muestran en la Tabla 7.1.1 (Etapa 4) (señalamos en negrita la decisión óptima en cada caso). 
Tabla 7.1.1: Ejemplo de la diligencia

\begin{tabular}{ccc}
\multicolumn{3}{c}{ Etapa 5} \\
\hline$e_{5}$ & $s_{5}$ & $c_{5}\left(e_{5}, x_{5}\right)$ \\
\hline \hline 10 & 12 & 6 \\
\hline 11 & 12 & 1 \\
\hline
\end{tabular}

\begin{tabular}{ccc}
\multicolumn{3}{c}{ Etapa 4} \\
\hline$e_{4}$ & $s_{4}$ & $c_{4}\left(e_{4}, x_{4}\right)$ \\
\hline \hline 7 & 10 & $4+6=10$ \\
& $\mathbf{1 1}$ & $3+1=\mathbf{4}$ \\
\hline 8 & 10 & $5+6=11$ \\
& $\mathbf{1 1}$ & $1+1=\mathbf{2}$ \\
\hline 9 & 10 & $2+6=8$ \\
& $\mathbf{1 1}$ & $3+1=\mathbf{4}$ \\
\hline
\end{tabular}

Etapa 3

\begin{tabular}{ccc}
\hline$e_{3}$ & $s_{3}$ & $c_{3}\left(e_{3}, x_{3}\right)$ \\
\hline \hline 5 & 7 & $5+4=9$ \\
& $\mathbf{8}$ & $5+2=\mathbf{7}$ \\
& 9 & $5+4=9$ \\
\hline 6 & 7 & $5+4=9$ \\
& $\mathbf{8}$ & $2+2=\mathbf{4}$ \\
& 9 & $1+4=5$ \\
\hline
\end{tabular}

Etapa 2

\begin{tabular}{ccc}
\hline$e_{2}$ & $s_{2}$ & $c_{2}\left(e_{2}, x_{2}\right)$ \\
\hline \hline 2 & 5 & $3+7=10$ \\
& $\mathbf{6}$ & $1+4=\mathbf{5}$ \\
\hline 3 & 5 & $5+7=12$ \\
& $\mathbf{6}$ & $3+4=\mathbf{7}$ \\
\hline 4 & 5 & $2+7=9$ \\
& $\mathbf{6}$ & $4+4=\mathbf{8}$ \\
\hline
\end{tabular}

Etapa 1

\begin{tabular}{ccc}
\hline$e_{1}$ & $s_{1}$ & $c_{1}\left(e_{1}, x_{1}\right)$ \\
\hline \hline 1 & $\mathbf{2}$ & $3+5=\mathbf{8}$ \\
& $\mathbf{3}$ & $1+7=\mathbf{8}$ \\
& 4 & $2+8=10$ \\
\hline
\end{tabular}


3. Si consideramos la Etapa 3, entonces estaremos en las ciudades 5 o 6, desde las que podemos ir a 1, 8, o 9 con los costes acumulados de las etapa 4 y 5 que se muestran en la Tabla 7.1.1 (Etapa 3).

4. Si consideramos la Etapa 2, entonces estaremos en las ciudades 2, 3 o 4, desde las que podemos ir a 5 o 6 con los costes acumulados de las etapa 3, 4 y 5 que se muestran en la Tabla 7.1.1 (Etapa 2).

5. Si consideramos la Etapa 1 entonces estaremos en la ciudad 1 desde la que podemos ir a 2, 3 o 4 con los costes acumulados de las etapa 2, 3, 4 y 5 que se muestran en la Tabla 7.1.1 (Etapa 1).

De modo que existen dos rutas óptimas que son

$$
R_{2}: 1 \rightarrow 2 \rightarrow 6 \rightarrow 8 \rightarrow 11 \rightarrow 12
$$

$\mathrm{y}$

$$
R_{3}: 1 \rightarrow 3 \rightarrow 6 \rightarrow 8 \rightarrow 11 \rightarrow 12,
$$

ambas con un coste asociado de 8 .

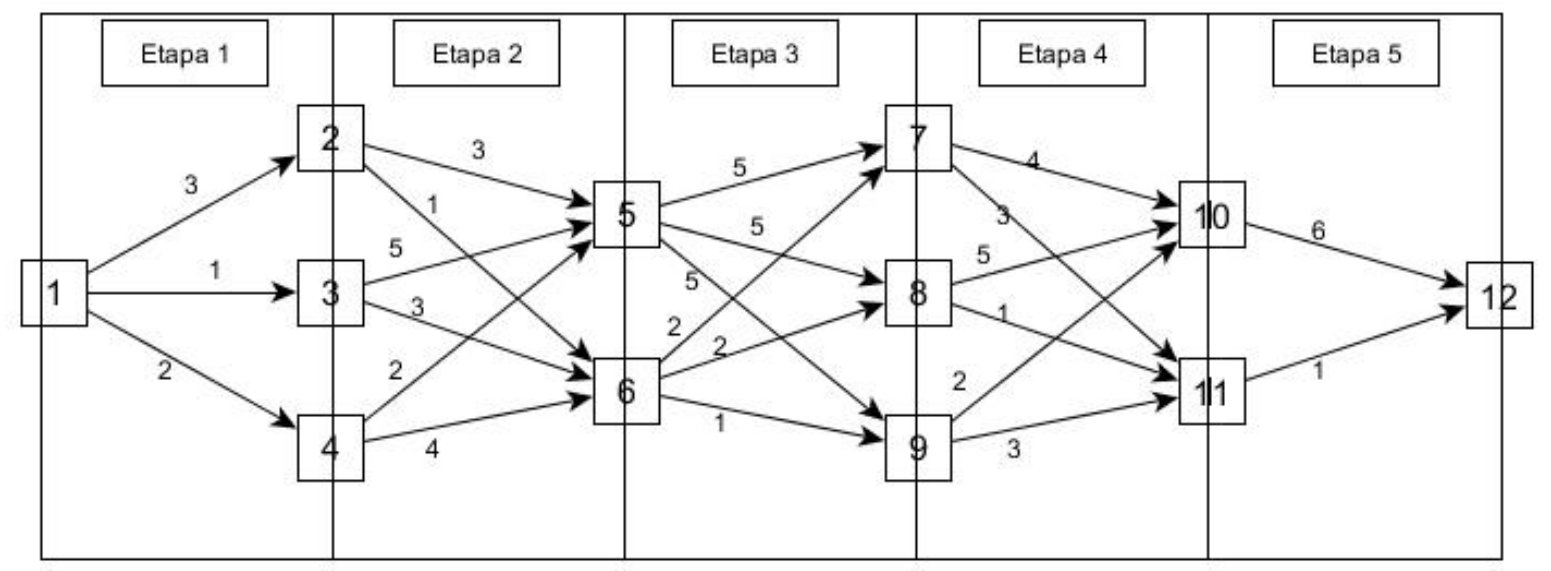

Figura 7.1.4: Etapas en el problema de la diligencia.

En la Sección 7.3 utilizaremos el paradigma de la programación dinámica para la selección óptima de salvaguardas que reduzcan la probabilidad de transmisión de fallos entre los activos a un nivel aceptable y con un coste mínimo en el grafo de activos que representa un sistema de información. 


\subsection{Tipos de salvaguardas y su efecto sobre el riesgo}

Supuesto realizado el análisis de riesgos (valorados los activos y sus grados de dependencias, identificadas las amenazas, su impacto y riesgo sobre los activos,...) el siguiente paso consiste en identificar las salvaguardas, y elegir un conjunto óptimo de ellas. Las salvaguardas son acciones sobre los activos que pueden reducir el riesgo ante las amenazas. Pueden ser de dos tipos [32, 66, 67, 72]:

- Salvaguardas preventivas: Reducen la frecuencia de la amenaza.

- Salvaguardas paliativas: Reducen el impacto de la amenaza sobre los activos (en cada componente).

En el caso de salvaguardas paliativas, el efecto provocado sobre una amenaza que se cierne sobre un activo tiene, al igual que el impacto de dicha amenaza, cinco componentes; mientras que si la salvaguarda es preventiva, el efecto tiene una única componente. Tales componentes serán términos lingüísticos de la escala predefinida en el análisis de riesgos.

La operación natural de sustracción en $[0,1]^{\mathcal{T F}}$ se define de la siguiente manera. Dados $\widetilde{A}=\left(a_{1}, a_{2}, a_{3}, a_{4}\right), \widetilde{B}=\left(b_{1}, b_{2}, b_{3}, b_{4}\right) \in[0,1]^{\mathcal{T F}}$ entonces

$$
\widetilde{A} \ominus \widetilde{B}=\left(a_{1}-b_{4}, a_{2}-b_{3}, a_{3}-b_{2}, a_{4}-b_{1}\right) .
$$

Nótese que la diferencia entre dos números borrosos trapezoidales de $[0,1]^{\mathcal{T F}}$ no tiene por qué pertenecer a dicho conjunto. Sin embargo, dado $\widetilde{A}=\left(a_{1}, a_{2}, a_{3}, a_{4}\right)$ se tiene que

$$
\left.\widetilde{1} \ominus \widetilde{A}=\left(1-a_{4}, 1-a_{3}, 1-a_{2}, 1-a_{1}\right)\right) \in[0,1]^{\mathcal{T F}},
$$

puesto que si $0 \leq a_{1} \leq a_{2} \leq a_{3} \leq a_{4} \leq 1$ se tiene que $0 \leq 1-a_{4} \leq 1-a_{3} \leq 1-a_{2} \leq$ $1-a_{1} \leq 1$.

El efecto $\widetilde{e} \in[0,1]^{\mathcal{T F}}$ de una salvaguarda $S_{i}$ preventiva sobre un activo $A$ y una amenaza dada provoca una reducción de la frecuencia de la amenaza de proporción $\widetilde{1} \ominus \widetilde{e}$, es decir, que la frecuencia de la amenaza dada con la salvaguarda $S_{i}$ se reduce al nivel

$$
\widetilde{f}^{\prime}=\widetilde{f} \otimes(\widetilde{1} \ominus \widetilde{e})
$$

La expresión es similar para una salvaguarda paliativa sobre cada componente de la degradación provocada por una amenaza sobre un activo: 
Si el efecto de una salvaguarda paliativa es $\left.\overrightarrow{\widetilde{e}}=\left(\widetilde{e}_{(1)}, \widetilde{e}_{(2)}, \widetilde{e}_{(3)}, \widetilde{e}_{(4)}, \widetilde{e}_{(5)}\right)\right)$ entonces la degradación provocada por la amenaza se reduce al nivel

$$
\widetilde{d}_{(i)}^{\prime}=\widetilde{d}_{(i)} \otimes\left(\widetilde{1} \ominus \widetilde{e}_{(i)}\right)
$$

Nótese que $\forall \widetilde{X} \in[0,1]^{\mathcal{T F}}$ se tiene que $\widetilde{X} \otimes \widetilde{A} \leq \widetilde{A} \forall \widetilde{A} \in[0,1]^{\mathcal{T F}}$. En particular $\widetilde{f^{\prime}} \leq \widetilde{f} \mathrm{y}$ $\widetilde{d}_{(i)}^{\prime} \leq \widetilde{d}_{(i)}$

El efecto provocado por varias salvaguardas sobre la degradación y la frecuencia de una amenaza sobre un activo $A$ se calcula aplicando sucesivamente las Ecuaciones 7.2.1 y 7.2 .2 .

Por lo tanto, si implementamos $m$ salvaguardas $S_{1}^{\text {Prev }}, \ldots, S_{m}^{\text {Prev }}$ preventivas, entonces la probabilidad de materialización de la amenaza se reduce al nivel $\widetilde{f}^{\prime}=\widetilde{f} \otimes\left[\stackrel{m}{\otimes}=1_{(}^{m}\left(\widetilde{1} \widetilde{e}_{k}\right)\right]$, siendo $\widetilde{e}_{k}$ el efecto provocado por la salvaguarda $S_{k}^{\text {Prev }}$ sobre la frecuencia de la amenaza.

Si implementamos $n$ salvaguardas paliativas $S_{1}^{\text {Pal }}, \ldots, S_{n}^{\text {Pal }}$, entonces la degradación sufrida por el activo atacado al materializarse la amenaza, se reduce al nivel $\widetilde{d}_{(i)}^{\prime}=\widetilde{d}_{(i)} \otimes$ $\left[\bigotimes_{j=1}^{n}\left(\widetilde{1} \ominus \widetilde{e}_{j_{(i)}}\right)\right]$ siendo $\widetilde{e}_{j_{(i)}}$ el efecto provocado por la salvaguarda paliativa $S_{j}^{P a l}$ en la componente $i$-ésima de la degradación de la amenaza.

\subsection{Minimización de la probabilidad de transmisión de fallos}

Por definición, una amenaza materializada sobre un activo de soporte $A_{i}$ puede transmitirse a un activo inferior $A_{j}$ (en dirección a los activos terminales) con probabilidad $\widetilde{D\left(A_{i}, A_{j}\right)}$. Así, los fallos generados por un activo atacado pueden alcanzar los activos terminales con graves consecuencias para la organización. Por tanto, una amenaza sobre el activo $A_{i}$ con probabilidad $\tilde{f}$ se puede considerar una amenaza sobre $A_{j}$ con probabilidad $\tilde{f} \otimes D \widetilde{D\left(A_{i}, A_{j}\right)}$. Entonces, un tercer tipo de salvaguardas son aquéllas que dificultan las transmisión de fallos entre activos. Tales salvaguardas no dependen de las amenazas consideradas, sino de los activos implicados.

Ahora bien, la probabilidad de transmisión de fallo $\overline{D\left(A_{i}, A_{j}\right)}$ es resultado de operaciones borrosas entre las probabilidades de transmisión de fallo de los activos intermedios que unen el activo de soporte atacado con otro activo. En cada uno de estos activos intermedios se pueden implementar salvaguardas que eviten o reduzcan la probabilidad 


\subsection{MINIMIZACIÓN DE LA PROBABILIDAD DE TRANSMISIÓN DE FALLOS}

de transmisión de fallo. De este modo, una vez identificadas estas salvaguardas, podemos considerar el problema de optimización consistente en elegir un paquete de éstas en cada activo con objeto de minimizar los costes, controlando los grados de dependencia indirectos $D \widetilde{D\left(A_{i}, A_{k}\right)}$ con el activo terminal $A_{k}$, para cualquier activo de soporte $A_{i}$. El problema consiste en elegir las salvaguardas más baratas de modo que la probabilidad de transmisión de fallos a los activos terminales sea asumible por la organización, con independencia de cuál sea el activo atacado.

Sea $S^{i, j}$ un conjunto de salvaguardas que dificultan la transmisión de fallo directo entre $A_{i}$ y $A_{j}$. Cada $S_{k}^{i, j} \in S^{i, j}$ tiene un coste monetario de $C_{k}^{i, j}$ y un efecto $\widetilde{e}_{k}^{i j}$ sobre $d \widetilde{\left(A_{i}, A_{j}\right)}$, de modo que esta probabilidad se reduce al nivel $d \widetilde{\left(A_{i}, A_{j}\right)} \otimes\left(1 \ominus \widetilde{e}^{i, j}\right)$.

El problema de mantener en un nivel asumible (bajo o muy bajo) la probabilidad de transmisión de fallos entre los activos de soporte y los activos terminales, con un coste mínimo, puede plantearse como:

$$
\begin{aligned}
& \text { Minimizar } \sum_{i, j} \sum_{k} C_{k}^{i, j} x_{k}^{i . j} \\
& \text { s.a } \\
& \quad \widetilde{D\left(A_{i}, A_{k}\right)} \leq \widetilde{U}_{i k} \forall i \forall k \\
& \quad \widetilde{x}_{k}^{i, j} \in\{\widetilde{0}, \widetilde{1}\} \forall i, j, k
\end{aligned}
$$

siendo $\widetilde{U}_{i k}$ un valor residual asumible por los decisores de SI, $x_{k}^{i, j}=1$ si seleccionamos la salvaguarda $S_{k}^{i, j}$, y donde $D \widetilde{D\left(A_{i}, A_{k}\right)}$ se recalcula sustituyendo los valores $d \widetilde{\left(A_{i}, A_{j}\right)}$ por los valores afectados por las salvaguardas seleccionadas entre dos activos consecutivos $\left.d \widetilde{\left(A_{i}, A_{j}\right.}\right) \otimes\left[\underset{k}{\otimes}\left(1-\widetilde{e}_{k}^{i, j}\right)\right]$.

Hemos de tener en cuenta que el grado de dependencia indirecto se calcula de modo recursivo siguiendo el algoritmo expuesto en la Sección 5.1. Así, el grado de dependencia de los activos de soporte más alejados de los terminales se puede calcular a partir del grado de dependencia de los activos más próximos. Por tanto, el problema se puede resolver por etapas, y se debe verificar el principio de optimalidad de la programación dinámica: Dada una secuencia óptima de decisiones, toda subsecuencia de ella es, a su vez, óptima.

Procedemos entonces de la siguiente manera:

- Sea $L_{0}$ el conjunto de activos terminales. 
- Consideramos el conjunto $L_{1}$ de todos los activos de soporte cuyos hijos estén en el conjunto $L_{0}$. Se eligen salvaguardas de modo que se minimicen los costes, manteniendo en un nivel asumible los grados de dependencia sobre sus hijos.

- Consideramos el conjunto $L_{2}$ de todos los activos de soporte cuyos hijos estén en el conjunto $L_{0} \cup L_{1}$. Se eligen salvaguardas de modo que se minimicen los costes, manteniendo en un nivel asumible los grados de dependencia sobre $L_{0}$. Nótese que los grados de dependencia indirectos de los hijos de $L_{2}$ sobre los activos terminales ya han sido determinados en la etapa anterior, por lo que sólo hay que calcular los grados de dependencia directos sobre los activos de $L_{0} \cup L_{1}$.

- Consideramos el conjunto $L_{n}$ de todos los activos de soporte cuyos hijos estén en el conjunto $L_{0} \cup L_{1} \cup \ldots \cup L_{n-1}$. Se eligen salvaguardas de modo que se minimicen los costes, manteniendo en un nivel asumible los grados de dependencia sobre $L_{0}$. De nuevo, nótese que los grados de dependencia indirectos de los hijos de $L_{n}$ sobre los activos terminales ya han sido determinados en la etapa anterior, por lo que sólo hay que calcular los grados de dependencia directos sobre los activos de $L_{0} \cup L_{1} \cup \ldots \cup L_{n-1}$.

En cada etapa podemos aplicar el algoritmo recocido simulado para realizar la selección óptima de las salvaguardas. El criterio de parada en cada etapa es un umbral de similitud a las cotas $\widetilde{U}_{i k}$. Cuando se alcanza este umbral se pasa a la siguiente etapa. El algoritmo finaliza cuando se han recorrido todas las etapas.

Otro criterio de parada puede ser un presupuesto prefijado. Cuando en una de las etapas se supera este presupuesto, finaliza la selección de salvaguardas pero se calcula el grado de dependencias de los activos restantes. Si los valores obtenidos son asumibles se finaliza. En caso contrario, se reinicia el algoritmo partiendo de una solución mejor (más barata) en $L_{1}$. Si no existe esta solución, se trata de mejorar la de $L_{2}$, y así sucesivamente. Si en ninguna etapa se consigue mejorar la solución obtenida anteriormente, entonces concluimos que el problema es infactible con el presupuesto prefijado. En esta variante se fija un límite de iteraciones en la búsqueda de una mejor solución dentro de cada etapa. 
Ejemplo.

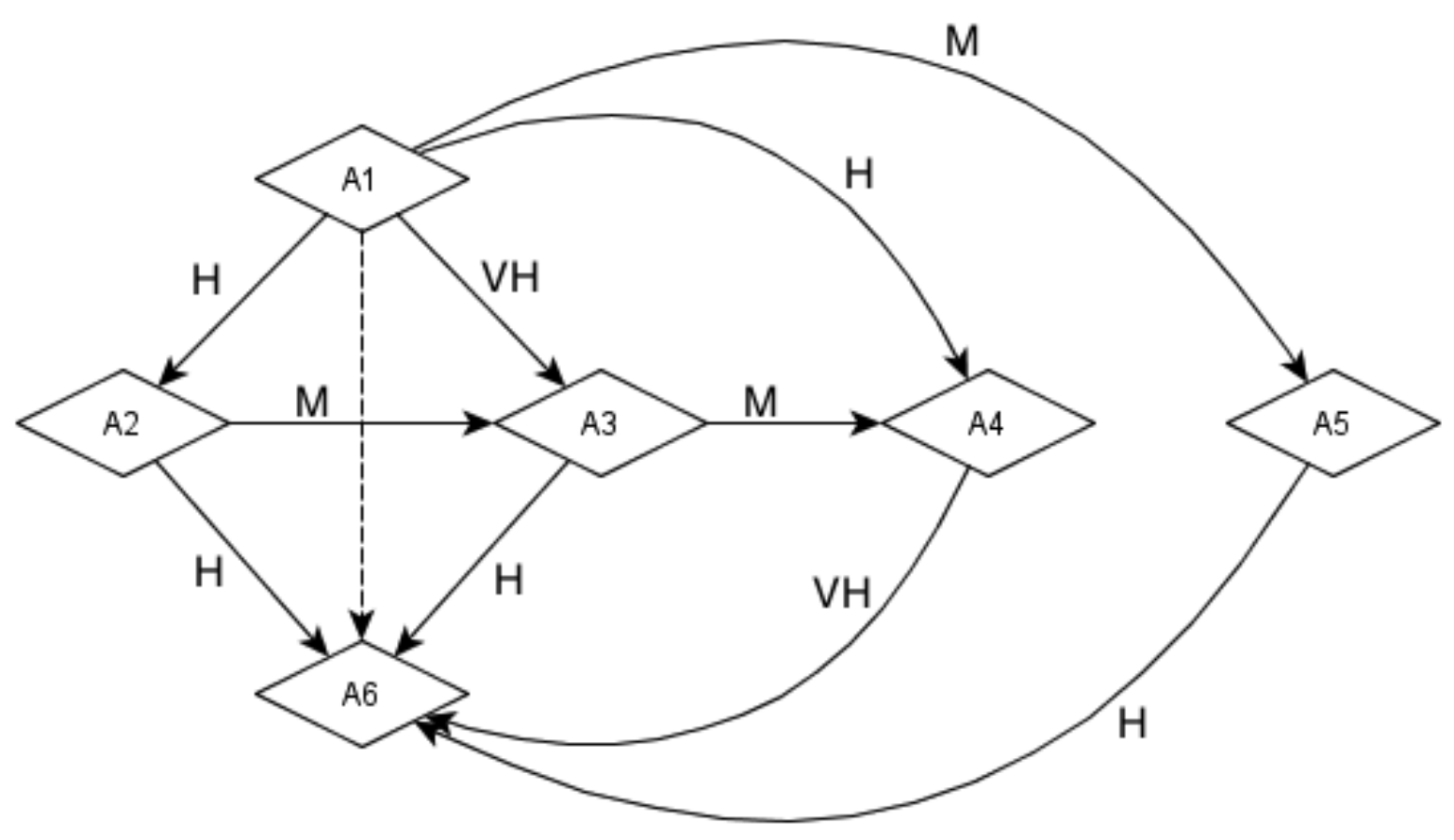

Figura 7.3.1: Estructura de dependencias para el ejemplo de minimización de la probabilidad de fallos

Basándonos en el ejemplo de la Figura 7.3.1, consideremos las salvaguardas de transmisión de fallos provocados en los activos de soporte dadas en las Tablas 7.3.1 a 7.3.5.

Tabla 7.3.1: Salvaguardas de transmisión de fallos provocados en $A_{1}$

\begin{tabular}{ccc|ccc}
\hline etiqueta & efecto & coste & etiqueta & efecto & coste \\
\hline \hline$S_{1}^{12}$ & $\mathrm{~L}$ & 100 & $S_{1}^{13}$ & $\mathrm{MH}$ & 356 \\
\hline$S_{2}^{12}$ & $\mathrm{M}$ & 300 & $S_{2}^{13}$ & $\mathrm{H}$ & 324 \\
\hline$S_{3}^{12}$ & $\mathrm{MH}$ & 550 & $S_{3}^{13}$ & $\mathrm{~L}$ & 110 \\
\hline$S_{4}^{12}$ & $\mathrm{M}$ & 430 & $S_{4}^{13}$ & $\mathrm{ML}$ & 345 \\
\hline$S_{5}^{12}$ & $\mathrm{ML}$ & 125 & $S_{5}^{13}$ & $\mathrm{VL}$ & 87 \\
\hline$S_{6}^{12}$ & $\mathrm{~L}$ & 240 & $S_{6}^{13}$ & $\mathrm{MH}$ & 345 \\
\hline$S_{7}^{12}$ & $\mathrm{VL}$ & 100 & $S_{7}^{13}$ & $\mathrm{M}$ & 200 \\
\hline$S_{8}^{12}$ & $\mathrm{MH}$ & 324 & & & \\
\hline$S_{9}^{12}$ & $\mathrm{VH}$ & 570 & & & \\
\hline
\end{tabular}


Tabla 7.3.2: Salvaguardas de transmisión de fallos provocados en $A_{1}$ (continuación)

\begin{tabular}{ccc|ccc}
\hline etiqueta & efecto & coste & etiqueta & efecto & coste \\
\hline \hline$S_{1}^{14}$ & $\mathrm{M}$ & 209 & $S_{1}^{15}$ & $\mathrm{M}$ & 230 \\
\hline$S_{2}^{14}$ & $\mathrm{M}$ & 267 & $S_{2}^{15}$ & $\mathrm{M}$ & 345 \\
\hline$S_{3}^{14}$ & $\mathrm{MH}$ & 342 & $S_{3}^{15}$ & $\mathrm{~L}$ & 187 \\
\hline$S_{4}^{14}$ & $\mathrm{VH}$ & 789 & $S_{4}^{15}$ & $\mathrm{M}$ & 321 \\
\hline$S_{5}^{14}$ & $\mathrm{M}$ & 234 & $S_{5}^{15}$ & $\mathrm{MH}$ & 345 \\
\hline$S_{6}^{14}$ & $\mathrm{M}$ & 356 & $S_{6}^{15}$ & $\mathrm{H}$ & 543 \\
\hline$S_{7}^{14}$ & $\mathrm{M}$ & 276 & $S_{7}^{15}$ & $\mathrm{MH}$ & 356 \\
\hline$S_{8}^{14}$ & $\mathrm{M}$ & 200 & $S_{8}^{15}$ & $\mathrm{M}$ & 206 \\
\hline$S_{9}^{14}$ & $\mathrm{H}$ & 467 & $S_{9}^{15}$ & $\mathrm{M}$ & 342 \\
\hline$S_{10}^{14}$ & $\mathrm{H}$ & 342 & & & \\
\hline$S_{11}^{14}$ & $\mathrm{~L}$ & 127 & & & \\
\hline$S_{12}^{14}$ & $\mathrm{M}$ & 207 & & & \\
\hline
\end{tabular}

Tabla 7.3.3: Salvaguardas de transmisión de fallos provocados en $A_{2}$

\begin{tabular}{ccc|ccc}
\hline etiqueta & efecto & coste & etiqueta & efecto & coste \\
\hline \hline$S_{1}^{23}$ & $\mathrm{M}$ & 356 & $S_{1}^{26}$ & $\mathrm{M}$ & 348 \\
\hline$S_{2}^{23}$ & $\mathrm{~L}$ & 87 & $S_{2}^{26}$ & $\mathrm{~L}$ & 187 \\
\hline$S_{3}^{23}$ & $\mathrm{ML}$ & 267 & $S_{3}^{26}$ & $\mathrm{ML}$ & 254 \\
\hline$S_{4}^{23}$ & $\mathrm{M}$ & 320 & $S_{4}^{26}$ & $\mathrm{ML}$ & 367 \\
\hline$S_{5}^{23}$ & $\mathrm{ML}$ & 156 & $S_{5}^{26}$ & $\mathrm{ML}$ & 567 \\
\hline$S_{6}^{23}$ & $\mathrm{M}$ & 320 & $S_{6}^{26}$ & $\mathrm{M}$ & 390 \\
\hline$S_{7}^{23}$ & $\mathrm{M}$ & 256 & $S_{7}^{26}$ & $\mathrm{ML}$ & 256 \\
\hline$S_{8}^{23}$ & $\mathrm{M}$ & 300 & $S_{8}^{26}$ & $\mathrm{M}$ & 307 \\
\hline$S_{9}^{23}$ & $\mathrm{~L}$ & 200 & $S_{9}^{26}$ & $\mathrm{~L}$ & 235 \\
\hline & & & $S_{10}^{26}$ & $\mathrm{ML}$ & 124 \\
\hline & & & $S_{11}^{26}$ & $\mathrm{M}$ & 400 \\
\hline & & $S_{12}^{26}$ & $\mathrm{~L}$ & 278 \\
\hline & & $S_{13}^{26}$ & $\mathrm{ML}$ & 260 \\
\hline
\end{tabular}




\subsection{MINIMIZACIÓN DE LA PROBABILIDAD DE TRANSMISIÓN DE FALLOS}

Tabla 7.3.4: Salvaguardas de transmisión de fallos provocados en $A_{3}$

\begin{tabular}{ccc|ccc}
\hline etiqueta & efecto & coste & etiqueta & efecto & coste \\
\hline \hline$S_{1}^{34}$ & $\mathrm{M}$ & 345 & $S_{1}^{36}$ & $\mathrm{M}$ & 267 \\
\hline$S_{2}^{34}$ & $\mathrm{H}$ & 650 & $S_{2}^{36}$ & $\mathrm{M}$ & 356 \\
\hline$S_{3}^{34}$ & $\mathrm{M}$ & 200 & $S_{3}^{36}$ & $\mathrm{M}$ & 378 \\
\hline$S_{4}^{34}$ & $\mathrm{M}$ & 367 & $S_{4}^{36}$ & $\mathrm{M}$ & 324 \\
\hline$S_{5}^{34}$ & $\mathrm{M}$ & 388 & $S_{5}^{36}$ & $\mathrm{M}$ & 345 \\
\hline$S_{6}^{34}$ & $\mathrm{H}$ & 453 & $S_{6}^{36}$ & $\mathrm{M}$ & 231 \\
\hline$S_{7}^{34}$ & $\mathrm{~L}$ & 189 & $S_{7}^{36}$ & $\mathrm{MH}$ & 453 \\
\hline$S_{8}^{34}$ & $\mathrm{~L}$ & 256 & & & \\
\hline$S_{9}^{34}$ & $\mathrm{M}$ & 345 & & & \\
\hline
\end{tabular}

Tabla 7.3.5: Salvaguardas de transmisión de fallos provocados en $A_{4}$ y $A_{5}$

\begin{tabular}{ccc|ccc}
\hline etiqueta & efecto & coste & etiqueta & efecto & coste \\
\hline \hline$S_{1}^{46}$ & $\mathrm{M}$ & 260 & $S_{1}^{56}$ & $\mathrm{M}$ & 200 \\
\hline$S_{2}^{46}$ & $\mathrm{M}$ & 245 & $S_{2}^{56}$ & $\mathrm{M}$ & 210 \\
\hline$S_{3}^{46}$ & $\mathrm{ML}$ & 170 & $S_{3}^{56}$ & $\mathrm{~L}$ & 120 \\
\hline$S_{4}^{46}$ & $\mathrm{M}$ & 256 & $S_{4}^{56}$ & $\mathrm{ML}$ & 234 \\
\hline$S_{5}^{46}$ & $\mathrm{M}$ & 367 & $S_{5}^{56}$ & $\mathrm{M}$ & 267 \\
\hline$S_{6}^{46}$ & $\mathrm{M}$ & 289 & $S_{6}^{56}$ & $\mathrm{MH}$ & 367 \\
\hline$S_{7}^{46}$ & $\mathrm{M}$ & 278 & $S_{7}^{56}$ & $\mathrm{MH}$ & 366 \\
\hline$S_{8}^{46}$ & $\mathrm{M}$ & 345 & $S_{8}^{56}$ & $\mathrm{M}$ & 254 \\
\hline$S_{9}^{46}$ & $\mathrm{M}$ & 240 & $S_{9}^{56}$ & $\mathrm{ML}$ & 145 \\
\hline$S_{10}^{46}$ & $\mathrm{MH}$ & 435 & $S_{10}^{56}$ & $\mathrm{~L}$ & 206 \\
\hline & & & $S_{11}^{56}$ & $\mathrm{M}$ & 306 \\
\hline & & & $S_{12}^{56}$ & $\mathrm{M}$ & 345 \\
\hline & & $S_{13}^{56}$ & $\mathrm{M}$ & 280 \\
\hline & & $S_{14}^{56}$ & $\mathrm{~L}$ & 178 \\
\hline & & $S_{15}^{56}$ & $\mathrm{MH}$ & 377 \\
\hline
\end{tabular}

Consideremos la cota $\widetilde{U}=(0,0,0.1,0.2)$ por debajo de la cual será asumible el grado de dependencia entre todos los activos y el activo terminal, y sea $\alpha=0.95$. Por tanto, la similitud del grado de dependencia resultante de aplicar las salvaguardas seleccionadas, con la cota $\widetilde{U}$, debe ser al menos 0.95 .

El espacio de soluciones en cada etapa se representa mediante matrices binarias. La fila $i$-ésima de la matriz corresponde a una selección de salvaguardas de $S_{i j}$ es decir, salvaguardas que evitan la transmisión de fallos del activo $i$-ésimo al resto de activos 
considerados en la etapa.

$D \widetilde{D\left(A_{i}, A_{j}\right)}$ se recalcula en cada iteración del algoritmo sustituyendo $d \widetilde{d\left(A_{i}, A_{j}\right)}$ por $\left.d \widetilde{\left(A_{i}, A_{j}\right.}\right) \otimes\left[\underset{n}{\otimes}\left(\widetilde{1}-\widetilde{e}_{n}^{i, j}\right)\right]$, y la función de similitud de números borrosos trapezoidales será la de Chen de 1996 [10],

$$
S\left(\left(a_{1}, a_{2}, a_{3}, a_{4}\right),\left(b_{1}, b_{2}, b_{3}, b_{4}\right)\right)=1-\frac{\sum\left|a_{i}-b_{i}\right|}{4},
$$

puesto que es la función de menor coste computacional de la literatura. Se pueden encontrar mejores funciones de similitud, a mayor coste computacional, en [11, 76, 23, 62, 78] y en esta tesis. Pasamos a describir cada una de las etapas.

- Etapa 1: En la Figura 7.3.1 se ajustan en primer lugar los grados de dependencia $D \widetilde{D\left(A_{4}, A_{6}\right)}$ y $D \widetilde{D\left(A_{5}, A_{6}\right)}$ con las restricciones $S\left(\widetilde{D D\left(A_{4}, A_{6}\right)}, \widetilde{U}\right) \geq 0.95 \mathrm{y}$ $\left.S\left(\widetilde{D\left(A_{5}, A_{6}\right.}\right), \widetilde{U}\right) \geq 0.95$, minimizando el coste.

Las soluciones sobre $A_{4}$ están representadas por una única fila $\left(x_{1}^{46}, x_{2}^{46}, \ldots, x_{10}^{46}\right)$, donde $x_{n}^{46}=1$ si se selecciona la salvaguarda $S_{n}^{46}$. Sobre $A_{5}$ la representación de la solución es similar.

Se parte de una solución binaria inicial y se aplica el algoritmo Recocido Simulado. La trayectoria de la función objetivo a lo largo del tiempo para la mejor solución encontrada se muestra en la Figura 7.3.2. La solución de esta primera etapa corresponde a las dos primeras filas de la Tabla 7.3.7.

En la Tabla 7.3.7 podemos ver las mejores soluciones obtenidas tras la ejecución del algoritmo varias veces en la minimización de $\widetilde{D\left(A_{5}, A_{6}\right)}$ con varios valores de $\alpha$. En ella se observa que, como cabría esperar, a medida que endurecemos la restrición y permitimos menores diferencias con la cota $\widetilde{U}$, el conjunto de salvaguardas resulta más caro de implementar. El mismo efecto se produce cuando utilizamos una cota $\widetilde{U}$ más precisa (con un soporte más reducido). Corresponde entonces a los decisores elegir valores más o menos altos de precisión para considerar asumible la dependencia entre activos y, por tanto, el riesgo soportado teniendo en cuenta este hecho.

- Etapa 2: Se ajustan $d \widetilde{d\left(A_{3,} A_{6}\right)}$ y $d \widetilde{d\left(A_{3,} A_{4}\right)}$ minimizando el coste bajo la restricción suavizada $S\left(\widetilde{D\left(A_{3}, A_{6}\right)}, \widetilde{U}\right) \geq 0.95$, donde 


$$
D \widetilde{D\left(A_{3}, A_{6}\right)}=d \widetilde{d\left(A_{3}, A_{6}\right)} \oplus\left(d \widetilde{d\left(A_{3}, A_{4}\right)} \otimes D \widetilde{D\left(A_{4}, A_{6}\right)}\right)
$$

Nótese que el valor $D \widetilde{D\left(A_{4}, A_{6}\right)}$ se ha calculado en la Etapa 1. En esta etapa la matriz de representación será

$$
\left(\begin{array}{c}
x_{1}^{36}, x_{2}^{36}, \ldots, x_{7}^{36}, 0,0 \\
x_{1}^{34}, x_{2}^{34}, \ldots \ldots \ldots, x_{9}^{34}
\end{array}\right) .
$$

A partir de esta representación, la función objetivo en la Etapa 2 es

$$
\begin{aligned}
\varphi= & \left(x_{1}^{36}, x_{2}^{36}, \ldots, x_{7}^{36}, 0,0\right)\left(C_{1}^{36}, C_{2}^{36}, \ldots, C_{7}^{36}, 0,0\right)^{T}+ \\
& +\left(x_{1}^{34}, x_{2}^{34}, \ldots \ldots \ldots, x_{9}^{34}\right)\left(C_{1}^{34}, C_{2}^{34}, \ldots, C_{9}^{34}\right)^{T} .
\end{aligned}
$$

La solución en esta etapa corresponde a la tercera fila de la Tabla 7.3.7.

- Etapa 3: Se ajusta $d \widehat{d\left(A_{2}, A_{3}\right)}$ y $d \widetilde{\left(A_{2}, A_{6}\right)}$ minimizando el coste bajo la restricción suavizada $S\left(\widetilde{D\left(A_{2}, A_{6}\right)}, \widetilde{U}\right) \geq 0.95$, donde

$$
D \widetilde{D\left(A_{2}, A_{6}\right)}=d \widetilde{\left(A_{2}, A_{6}\right)} \oplus\left(d \widetilde{d\left(A_{2}, A_{3}\right)} \otimes D \widetilde{D\left(A_{3}, A_{6}\right)}\right)
$$

Nótese que el valor $D \widetilde{D\left(A_{3}, A_{6}\right)}$ se ha calculado en la Etapa 2. La representación de la solución en esta etapa es similar a la anterior, y la solución óptima corresponde a la cuarta fila de la Tabla 7.3.7.

- Finalmente, se ajustan $d d \widetilde{\left(A_{1}, A_{2}\right)}, d d \widetilde{\left(A_{1}, A_{3}\right)}, d d \widetilde{\left(A_{1}, A_{4}\right)}$ y $d d \widetilde{\left(A_{1}, A_{5}\right)}$ minimizando el coste bajo la restricción

$$
\begin{gathered}
\left.D \widetilde{D\left(A_{1},\right.} A_{6}\right)=\left(d d \widetilde{\left(A_{1}, A_{2}\right) \otimes D} \widetilde{D\left(A_{2}, A_{6}\right)}\right) \oplus \\
\left(d \widetilde{d\left(A_{1}, A_{3}\right)} \otimes D \widetilde{D\left(A_{3}, A_{6}\right)}\right) \oplus \\
\left(d d \widetilde{\left(A_{1}, A_{4}\right)} \otimes D \widetilde{D\left(A_{4}, A_{6}\right)}\right) \oplus \\
\left(d \widetilde{\left(A_{1}, A_{5}\right)} \otimes D \widetilde{D\left(A_{5}, A_{6}\right)}\right) \leq \widetilde{U}_{16} .
\end{gathered}
$$

Hemos de tener en cuenta que los valores de los grados de dependencia $\widetilde{D\left(A_{1}, A_{6}\right)}$, $D \widetilde{D\left(A_{2}, A_{6}\right)}, D \widetilde{D\left(A_{3}, A_{6}\right)}, D \widetilde{D\left(A_{4}, A_{6}\right)}$ y $D \widetilde{D\left(A_{5}, A_{6}\right)}$ han sido calculados en las etapas anteriores. La solución en esta etapa corresponde a la quinta fila de la Tabla 7.3.7. 


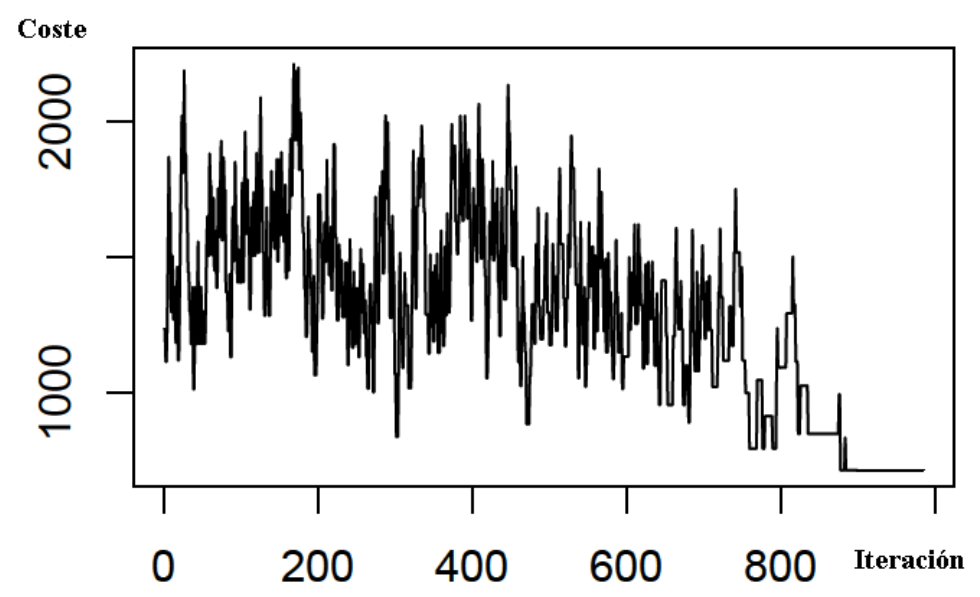

Figura 7.3.2: Trayectoria de la función objetivo del recocido simulado

Tabla 7.3.6: Coste de las mejores soluciones encontradas para $\widetilde{D\left(A_{5}, A_{6}\right)}$ variando el valor de $\alpha$.

\begin{tabular}{cccc}
\hline$\alpha$ & $\overrightarrow{D D\left(A_{5}, A_{6}\right) \text { resultante }}$ & Similitud & Coste \\
\hline \hline 0.80 & $(0.06,0.21,0.27,0.47)$ & 0.81 & 554 \\
\hline 0.9 & $(0.02,0.09,0.13,0.30)$ & 0.93 & 755 \\
\hline 0.95 & $(0.01,0.07,0.11,0.28)$ & 0.95 & 818 \\
\hline 0.98 & $(0.00,0.03,0.06,0.19)$ & 0.98 & 1176 \\
\hline
\end{tabular}

Tabla 7.3.7: Soluciones óptimas encontradas y coste económico de la implementación.

\begin{tabular}{ccc}
\hline Activo & Solución & Coste \\
\hline \hline$A_{5}$ & $\mathrm{~S}_{6}^{56}, S_{9}^{56}, S_{11}^{56}$ & 818 \\
\hline$A_{4}$ & $\mathrm{~S}_{6}^{46}, S_{9}^{46}, S_{10}^{46}$ & 935 \\
\hline$A_{3}$ & $\mathrm{~S}_{3}^{34}, S_{4}^{36}, S_{6}^{36}, S_{7}^{36}$ & 1208 \\
\hline$A_{2}$ & $\mathrm{~S}_{8}^{23}, S_{9}^{23}, S_{7}^{26}, S_{10}^{26}$ & 808 \\
\hline$A_{1}$ & $\mathrm{~S}_{1}^{12}, S_{3}^{12}, S_{9}^{12}, S_{1}^{13}, S_{2}^{13}, S_{10}^{14}, S_{11}^{14}, S_{7}^{15}$ & 2479 \\
\hline \multicolumn{3}{c}{ Coste total } \\
\hline
\end{tabular}

Tabla 7.3.8: Grado de dependencia de cada activo tras la implementación de salvaguardas

\begin{tabular}{cc}
\hline Activo & $\overline{D D\left(A_{k}, A_{6}\right)}$ \\
\hline \hline$A_{5}$ & $(0.010,0.071,0.110,0.281)$ \\
\hline$A_{4}$ & $(0.012,0.062,0.089,0.216)$ \\
\hline$A_{3}$ & $(0.010,0.067,0.105,0.293)$ \\
\hline$A_{2}$ & $(0.004,0.047,0.084,0.323)$ \\
\hline$A_{1}$ & $(0.001,0.029,0.058,0.262)$ \\
\hline
\end{tabular}


En la Figura 7.3.3 se compara el riesgo soportado por el activo $A_{1}$ antes y después de la implementación de las salvaguardas.

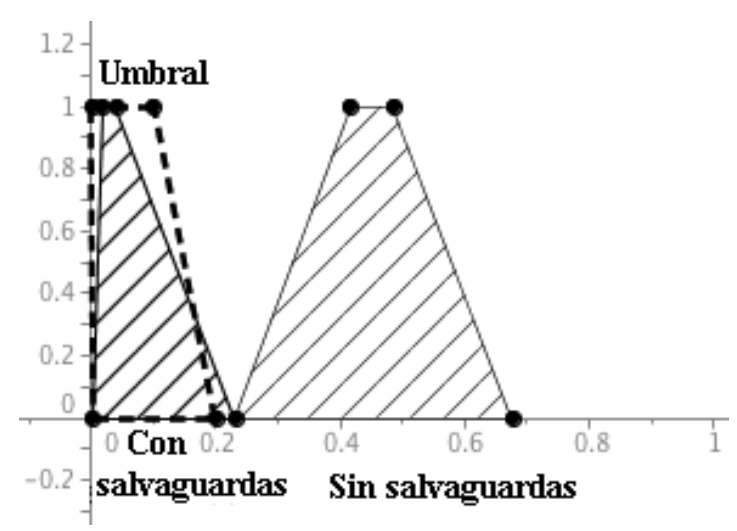

Figura 7.3.3: Riesgo en cada componente de $A_{1}$ antes y después de la implementación de salvaguardas

\subsection{Selección óptima de salvaguardas en la gestión de riesgos}

También es posible reducir el riesgo del sistema bajo un presupuesto dado.

Denotemos los conjuntos de salvaguardas por:

- $S_{t}^{i j}=t$-ésima salvaguarda de transmisión de fallos entre los activos $A_{i}$ y $A_{j}, t=$ $1, \ldots, n_{i j}$. Su efecto sobre el grado de dependencia $d \widetilde{\left(A_{i}, A_{j}\right)}$ es $\widetilde{e}^{S_{t}^{i j}}$.

- $S_{t}^{T_{r}^{i} \text { prev }}=t$-ésima salvaguarda preventiva sobre la $r$-ésima amenaza sobre el activo $A_{i}, t=1, \ldots, m_{r i}^{p r e v}$. Su efecto sobre la frecuencia de la amenaza $T_{r}^{i}$ es $\widetilde{e}^{S_{t}^{T_{r}^{i} \text { prev }}}$.

- $S_{t}^{T_{r}^{i} p a l}=t$-ésima salvaguarda paliativa sobre la $r$-ésima amenaza sobre el activo $A_{i}$, $t=1, \ldots, m_{r i}^{p a l}$. Su efecto sobre la frecuencia de la amenaza $T_{r}^{i}$ es $\widetilde{e}^{S_{t}^{T_{r}^{i}} \text { pal }}$.

Podemos seleccionar distintos paquetes de salvaguardas para reducir el riesgo. Estos paquetes serán cadenas binarias $X_{i j}=\left(x_{t}\right)_{t=1}^{n_{i j}}, X_{t}^{r i-p r e v}=\left(x_{t}\right)_{t=1}^{m_{r i}^{p r e v}}, X_{t}^{r i-p a l}=\left(x_{t}\right)_{t=1}^{m_{r i}^{p a l}}$, donde $x_{t}=1$ si se selecciona la $t$-ésima salvaguarda en cada caso.

El problema consiste en minimizar el máximo riesgo soportado por el sistema sujeto a un presupuesto económico $C$ : 


$$
\begin{aligned}
& \text { Minimizar } \max _{T_{r}^{i}, l}\left\{\widetilde{R}_{(l)}^{T_{r}^{i}}\right\} \\
& \text { s.a } \\
& \qquad \sum_{i j} X_{i j} C_{i j}^{\prime}+\sum_{r i-p r e v} X_{t}^{r i-p r e v} C_{r i-p r e v}^{\prime}+\sum_{r i-p a l} X_{t}^{r i-p a l} C_{r i-p a l}^{\prime} \leq C,
\end{aligned}
$$

donde $C_{i j}, C_{r i-p r e v}$ y $C_{r i-p a l}$ son los vectores que recogen los costes de las salvaguardas consideradas.

Se trata de un problema combinatorio cuya complejidad crece con la dimensión de la red de activos, ya que en cada arco se pueden considerar a su vez diferentes paquetes de salvaguardas, y las salvaguardas seleccionadas sobre los arcos más cercanos a los activos terminales reducen también la probabilidad de transmisión de fallos de los activos más alejados.

Concretamente, se tienen $2^{\sum_{i, k} n_{i k}+\sum_{i, j} m_{i j}^{p r}+\sum_{i, j} m_{i j}^{p r}}$ soluciones factibles y $5 \times s$ elementos de riesgo en la funcón objetivo (donde $s$ es el número total de amenazas consideradas). Por tanto, el uso nuevamente de técnicas metaheurísticas se hace indispensable.

Por otro lado, a diferencia del problema planteado en la sección anterior, la función objetivo dada necesita de una noción de orden total, de la cual carecen los números borrosos.

La definición de índices adecuados para ordenar números borrosos trapezoidales ha sido una cuestión trascendental en la historia de la lógica borrosa dada su utilidad en los sistemas de ayuda a la decisión en este ambiente. En [6, 13, 75] se dan clasificaciones de más de una treintena de métodos de ordenación de números borrosos trapezoidales. La razón de tal cantidad de métodos es que todos ellos tienen algún inconveniente. En este trabajo utilizamos el índice de Murakami et al. [44] que consiste en calcular el centroide de los números borrosos comparados:

Si $\widetilde{A}=\left(a_{1}, a_{2}, a_{3}, a_{4}\right)$, su centro de gravedad es el punto

$$
\bar{Y}_{\widetilde{A}}=\frac{\left(\frac{a_{3}-a_{2}}{a_{4}-a_{1}}+2\right)}{6}, \quad \bar{X}_{\widetilde{A}}=\bar{Y}_{\widetilde{A}}\left(a_{3}-a_{2}\right)+\left(1-\bar{Y}_{\widetilde{A}}\right)\left(a_{4}-a_{1}\right)
$$

La ordenación de Murakami consiste en comparar primero las abscisas de los centroides. Es mayor el número borroso cuyo centroide tiene mayor abscisa. Si ambas abscisas son iguales, entonces es mayor el que tiene el centroide con la mayor ordenada. 


\section{Ejemplo}

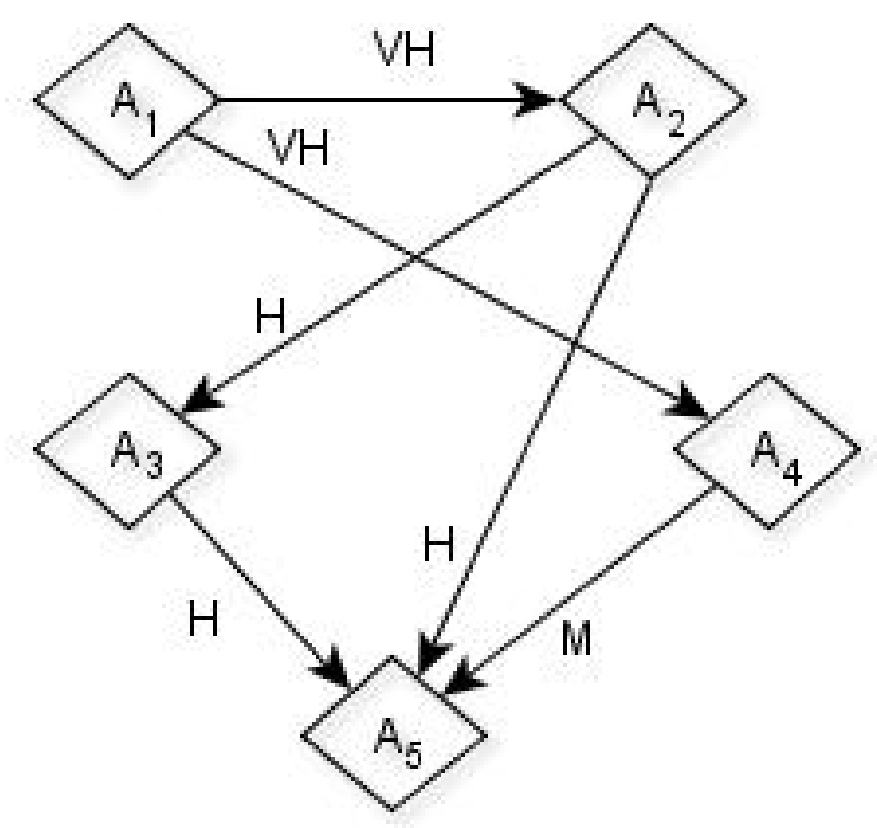

Figura 7.4.1: Red de activos

Consideremos el SI de la Figura 7.4.1 en el que el activo $A_{5}$ es el terminal y su valor es $\overrightarrow{v_{5}}=(10000,15000,20000,25000),(18000,20000,23000,30000),(12000,15000,26000,30000)$.

En primer lugar, la probabilidad de transmisión de fallos de cada activo de soporte con respecto al activo terminal es:

- $\left.\left.D \widetilde{\left(A_{4}, A_{5}\right.}\right)=d \widetilde{\left(A_{4}, A_{5}\right.}\right)=M=(0.35,0.45,0.65,0.75)$,

- $\left.D \widetilde{\left(A_{3}, A_{5}\right.}\right)=d\left(\widetilde{A_{3}, A_{5}}\right)=H=(0.75,0.85,0.95,1)$,

- $\left.\left.\left.\left.D \widetilde{\left(A_{2}, A_{5}\right.}\right)=d \widetilde{\left(A_{2}, A_{5}\right.}\right) \uplus\left(d \widetilde{\left(A_{2}, A_{3}\right.}\right) \otimes D \widetilde{\left(A_{3}, A_{5}\right.}\right)\right)=H \uplus(H \otimes H)=(0.93,0.97,0.99,1)$,

- $\left.\left.\left.\left.D \widetilde{\left(A_{1}, A_{5}\right.}\right)=\left(d \widetilde{\left(A_{1}, A_{4}\right.}\right) \otimes D \widetilde{\left(A_{4}, A_{5}\right.}\right)\right) \uplus\left(d \widetilde{\left(A_{1}, A_{2}\right)} \otimes D \widetilde{\left(A_{2}, A_{5}\right.}\right)\right)=(V H \otimes M) \uplus$ $(V H \otimes(0.93,0.97,0.99,1))=(0.92,0.98,0.99,1)$.

Consideremos las amenazas de la Tabla 7.4.1 sobre los activos del sistema. El riesgo provocado por dichas amenazas se muestran en la Tabla 7.4.2. 
Tabla 7.4.1: Amenazas sobre activos

\begin{tabular}{cccccc}
\hline Activo & Amenaza & Probabilidad & Degradación & & \\
\hline \hline & & & $\mathrm{C}$ & $\mathrm{I}$ & $\mathrm{D}$ \\
\hline$A_{1}$ & $T_{1}^{1}$ & $\mathrm{H}$ & $\mathrm{M}$ & $\mathrm{H}$ & $\mathrm{MH}$ \\
\hline$A_{2}$ & $T_{1}^{2}$ & $\mathrm{M}$ & $\mathrm{H}$ & $\mathrm{M}$ & $\mathrm{MH}$ \\
& $T_{2}^{2}$ & $\mathrm{H}$ & $\mathrm{M}$ & $\mathrm{M}$ & $\mathrm{M}$ \\
\hline$A_{3}$ & $T_{1}^{3}$ & $\mathrm{MH}$ & $\mathrm{H}$ & $\mathrm{H}$ & $\mathrm{M}$ \\
\hline$A_{4}$ & $T_{1}^{4}$ & $\mathrm{H}$ & $\mathrm{H}$ & $\mathrm{MH}$ & $\mathrm{M}$ \\
\hline
\end{tabular}

Tabla 7.4.2: Riesgo sobre $A_{5}$

\begin{tabular}{cccc}
\hline & $\mathrm{C}$ & $\mathrm{I}$ & $\mathrm{D}$ \\
\hline \hline$T_{1}^{1}$ & $(2898,5622,75,13449,15,19500)$ & $(6210,10620,75,23230,35,30000)$ & $(4554,8121,75,18339,75,25500)$ \\
\hline$T_{1}^{2}$ & $(2929,5,5565,375,13449,15,19500)$ & $(1367,1,2946,375,7786,35,12675)$ & $(2148,3,4255,875,10617,75,16575)$ \\
\hline$T_{2}^{2}$ & $(2929,5,5565,375,13449,15,19500)$ & $(2929,5,5565,375,13449,15,19500)$ & $(2929,5,5565,375,13449,15,19500)$ \\
\hline$T_{1}^{3}$ & $(3712,5,7044,375,17598,75,25500)$ & $(3712,5,7044,375,17598,75,25500)$ & $(1732,5,3729,375,10188,75,16575)$ \\
\hline$T_{1}^{4}$ & $(2362,5,4876,875,12905,75,19500)$ & $(1732,5,3729,375,10188,75,16575)$ & $(1102,5,2581,875,7471,75,12675)$ \\
\hline
\end{tabular}

Consideremos las salvaguardas de transmisión de fallos de la Tabla 7.4.3, las salvaguardas paliativas de la Tabla 7.4.5 y las preventivas de la Tabla 7.4.4 sobre las amenazas de la Tabla 7.4.1.

El problema consiste en seleccionar paquetes de salvaguardas de cada tipo de modo que se minimice:

Tabla 7.4.3: Salvaguardas de transmisión de fallos

\begin{tabular}{ccc|ccc|ccc|ccc}
\hline & $A_{4}$ & \multicolumn{3}{|c|}{$A_{3}$} & \multicolumn{3}{c}{$A_{2}$} & \multicolumn{3}{c}{$A_{1}$} \\
\hline Etiqueta & Efecto & Coste & Etiqueta & Efecto & Coste & Etiqueta & Efecto & Coste & Etiqueta & Efecto & Coste \\
\hline \hline$S_{1}^{45}$ & $\mathrm{M}$ & 205 & $S_{1}^{35}$ & $\mathrm{M}$ & 198 & $S_{1}^{25}$ & $\mathrm{M}$ & 203 & $S_{1}^{14}$ & $\mathrm{M}$ & 178 \\
\hline$S_{2}^{45}$ & $\mathrm{~L}$ & 124 & $S_{2}^{35}$ & $\mathrm{~L}$ & 100 & $S_{2}^{25}$ & $\mathrm{M}$ & 198 & $S_{2}^{14}$ & $\mathrm{M}$ & 160 \\
\hline$S_{3}^{45}$ & $\mathrm{ML}$ & 230 & $S_{3}^{35}$ & $\mathrm{M}$ & 123 & $S_{3}^{25}$ & $\mathrm{~L}$ & 170 & $S_{3}^{14}$ & $\mathrm{~L}$ & 120 \\
\hline$S_{4}^{45}$ & $\mathrm{M}$ & 189 & $S_{4}^{35}$ & $\mathrm{M}$ & 167 & $S_{1}^{23}$ & $\mathrm{~L}$ & 143 & $S_{4}^{14}$ & $\mathrm{~L}$ & 105 \\
\hline$S_{5}^{45}$ & $\mathrm{~L}$ & 104 & $S_{5}^{35}$ & $\mathrm{~L}$ & 89 & $S_{2}^{23}$ & $\mathrm{M}$ & 178 & $S_{1}^{12}$ & $\mathrm{~L}$ & 120 \\
\hline$S_{6}^{45}$ & $\mathrm{M}$ & 167 & $S_{6}^{35}$ & $\mathrm{M}$ & 178 & $S_{3}^{23}$ & $\mathrm{M}$ & 154 & $S_{2}^{12}$ & $\mathrm{M}$ & 180 \\
\hline$S_{7}^{45}$ & $\mathrm{M}$ & 178 & $S_{7}^{35}$ & $\mathrm{M}$ & 209 & $S_{4}^{23}$ & $\mathrm{M}$ & 190 & $S_{3}^{12}$ & $\mathrm{~L}$ & 104 \\
\hline$S_{8}^{45}$ & $\mathrm{~L}$ & 98 & $S_{8}^{35}$ & $\mathrm{~L}$ & 100 & $S_{5}^{23}$ & $\mathrm{~L}$ & 102 & $S_{4}^{12}$ & $\mathrm{M}$ & 200 \\
\hline
\end{tabular}




\subsection{SELECCIÓN ÓPTIMA DE SALVAGUARDAS EN LA GESTIÓN DE RIESGOS}

Tabla 7.4.4: Salvaguardas paliativas

\begin{tabular}{|c|c|c|c|c|c|c|c|c|c|}
\hline \multirow[t]{2}{*}{ Etiqueta } & \multicolumn{3}{|c|}{$\widetilde{e}$} & \multirow[t]{2}{*}{ Coste } & \multirow[t]{2}{*}{ Etiqueta } & \multicolumn{3}{|c|}{$\widetilde{e}$} & \multirow[t]{2}{*}{ Coste } \\
\hline & $\mathrm{C}$ & I & $\mathrm{D}$ & & & $\mathrm{C}$ & I & $\mathrm{D}$ & \\
\hline$S_{1}^{T_{1}^{1} p a l}$ & $\mathrm{H}$ & $\mathrm{H}$ & $\mathrm{H}$ & 520 & $S_{1}^{T_{1}^{2} p a l}$ & $\mathrm{H}$ & $\mathrm{H}$ & $\mathrm{H}$ & 496 \\
\hline$S_{2}^{T_{1}^{1} p a l}$ & $\mathrm{M}$ & $\mathrm{L}$ & M & 250 & $S_{2}^{T_{1}^{2} p a l}$ & $\mathrm{VL}$ & $\mathrm{L}$ & $\mathrm{ML}$ & 110 \\
\hline$S_{3}^{T_{1}^{1} p a l}$ & $\mathrm{~L}$ & $\mathrm{~L}$ & $\mathrm{VL}$ & 100 & $S_{3}^{T_{1}^{2} p a l}$ & ML & M & $\mathrm{L}$ & 78 \\
\hline$S_{4}^{T_{1}^{1} p a l}$ & ML & $\mathrm{VL}$ & $\mathrm{L}$ & 96 & $S_{1}^{T_{2}^{2} p a l}$ & $\mathrm{M}$ & $\mathrm{L}$ & $\mathrm{M}$ & 195 \\
\hline$S_{5}^{T_{1}^{1} p a l}$ & $\mathrm{VL}$ & $\mathrm{L}$ & ML & 110 & $S_{2}^{T_{2}^{2} p a l}$ & $\mathrm{~L}$ & $\mathrm{~L}$ & VL & 89 \\
\hline$S_{6}^{T_{1}^{1} p a l}$ & ML & M & $\mathrm{L}$ & 78 & $S_{3}^{T_{2}^{2} p a l}$ & ML & $\mathrm{VL}$ & $\mathrm{L}$ & 56 \\
\hline$S_{1}^{T_{1}^{3} p a l}$ & $\mathrm{H}$ & $\mathrm{H}$ & $\mathrm{H}$ & 535 & $S_{1}^{T_{1}^{4} p a l}$ & $\mathrm{H}$ & $\mathrm{H}$ & $\mathrm{H}$ & 539 \\
\hline$S_{2}^{T_{1}^{3} p a l}$ & $\mathrm{~L}$ & $\mathrm{~L}$ & $\mathrm{VL}$ & 89 & $S_{2}^{T_{1}^{4} p a l}$ & $\mathrm{~L}$ & $\mathrm{~L}$ & $\mathrm{VL}$ & 110 \\
\hline$S_{3}^{T_{1}^{3} p a l}$ & $\mathrm{H}$ & $\mathrm{H}$ & $\mathrm{H}$ & 670 & $S_{3}^{T_{1}^{4} p a l}$ & ML & $\mathrm{H}$ & $\mathrm{L}$ & 478 \\
\hline$S_{4}^{T_{1}^{3} p a l}$ & ML & $\mathrm{H}$ & $\mathrm{L}$ & 537 & $S_{3}^{T_{1}^{4} p a l}$ & $\mathrm{ML}$ & $\mathrm{H}$ & $\mathrm{L}$ & 495 \\
\hline$S_{5}^{T_{1}^{3} p a l}$ & $\mathrm{H}$ & $\mathrm{L}$ & ML & 477 & $S_{4}^{T_{1}^{4}} \mathrm{pal}$ & $\mathrm{H}$ & $\mathrm{H}$ & $\mathrm{H}$ & 689 \\
\hline
\end{tabular}

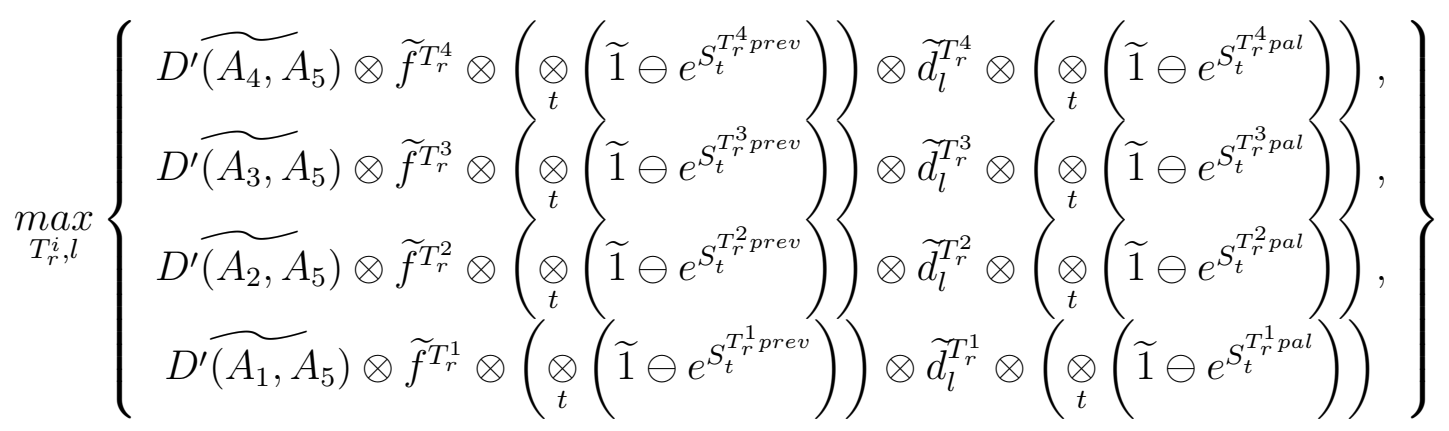

s.a

$$
\sum_{i j} X_{i j} C_{i j}^{\prime}+\sum_{r i-p r e v} X_{t}^{r i-p r e v} C_{r i-p r e v}^{\prime}+\sum_{r i-p a l} X_{t}^{r i-p a l} C_{r i-p a l}^{\prime} \leq C
$$

donde $\widetilde{D^{\prime}\left(A_{i}, A_{5}\right)}$ resulta de recalcular $\left.\widetilde{D\left(A_{i}, A_{5}\right.}\right)$ multiplicando el valor inicial de cada arco por la reducción provocada por el efecto de las salvaguardas de transmisión de fallos en dicho arco.

La función objetivo considera $3 \times 5=15$ elementos de riesgos, y el tamaño del conjunto de soluciones factibles es $2^{32+22+16}=1.18 \times 10^{21}$.

Consideraremos un presupuesto de 5000 unidades monetarias.

La solución inicial se obtiene generando aleatoriamente un paquete de salvaguardas de cada tipo para cada amenaza. Un paquete de salvaguardas de transmisión de fallos está a 
Tabla 7.4.5: Salvaguardas preventivas

\begin{tabular}{ccc|ccc}
\hline Etiqueta & $\widetilde{e}$ & Coste & Tag & $\widetilde{e}$ & Coste \\
\hline \hline$S_{1}^{T_{1}^{1}}$ prev & $\mathrm{H}$ & 367 & $S_{1}^{T_{1}^{2}}$ prev & $\mathrm{H}$ & 470 \\
\hline$S_{2}^{T_{1}^{1}}$ prev & $\mathrm{H}$ & 485 & $S_{2}^{T_{1}^{2}}$ prev & $\mathrm{ML}$ & 89 \\
\hline$S_{3}^{T_{1}^{1} \text { prev }}$ & $\mathrm{ML}$ & 100 & $S_{1}^{T_{2}^{2} \text { prev }}$ & $\mathrm{L}$ & 56 \\
\hline$S_{4}^{T_{1}^{1}}$ prev & $\mathrm{ML}$ & 120 & $S_{2}^{T_{2}^{2} \text { prev }}$ & $\mathrm{ML}$ & 98 \\
\hline \hline$S_{1}^{T_{1}^{3} \text { prev }}$ & $\mathrm{ML}$ & 125 & $S_{1}^{T_{1}^{4} \text { prev }}$ & $\mathrm{M}$ & 127 \\
\hline$S_{2}^{T_{1}^{3} \text { prev }}$ & $\mathrm{H}$ & 498 & $S_{2}^{T_{1}^{4}}$ prev & $\mathrm{ML}$ & 100 \\
\hline$S_{3}^{T_{1}^{3} \text { prev }}$ & $\mathrm{L}$ & 84 & $S_{3}^{T_{1}^{4} \text { prev }}$ & $\mathrm{L}$ & 67 \\
\hline$S_{4}^{T_{1}^{3} \text { prev }}$ & $\mathrm{L}$ & 75 & $S_{4}^{T_{1}^{4}}$ prev & $\mathrm{L}$ & 54 \\
\hline
\end{tabular}

su vez formado por diferentes paquetes en cada arco. El coste total de la solución inicial, así como de las vecinas consideradas, debe ser obviamente inferior a 5000.

El entorno de una solución está formada por todas las soluciones factibles cuyos paquetes de salvaguardas se diferencien en a lo sumo una salvaguarda.

La temperatura inicial, como en la Sección anterior, se calcula de modo que la probabilidad de aceptación de cualquier solución peor que la inicial sea al menos 0.9 , independientemente de lo mala que sea la solución del entorno, es decir:

$$
e^{-\left(f(y)-f\left(x_{0}\right)\right) / T_{0}} \geq 0,9 \forall y \in N\left(x_{0}\right),
$$

para lo cual basta calcular una cota superior $M \geq\left(f(y)-f\left(x_{0}\right)\right.$.

En virtud del criterio de ordenación de Murakami utilizado, podemos considerar $M=$ $\max _{l, i, r}\left\{\bar{X}_{\widetilde{R}_{(l)}^{T_{i}^{i}}}\right\}$, que obviamente se alcanza cuando no se considera salvaguarda alguna sobre el sistema: $M=19077$ y $T_{0}=181064$.

La forma de enfriamiento consiste en reducir la temperatura un $10 \%$ cada 20 iteraciones del algoritmo, es decir, partiendo de $T=T_{0}$, la temperatura se actualiza en $T=0.9 T$ cada 20 iteraciones. Finalmente, el criterio de parada es que no se haya mejorado durante el último $30 \%$ de la iteraciones totales.

En la Figura 7.4.2 puede verse la trayectoria de la abscisa del centroide del riesgo máximo, que llega a reducirse al nivel 4044.302 (antes de la implementación de salvaguardas es 19077). En la Tabla 7.4.6 puede verse la solución obtenida, cuyo coste asciende a 4850 unidades monetarias. Finalmente, el riesgo una vez implementadas las salvaguardas 


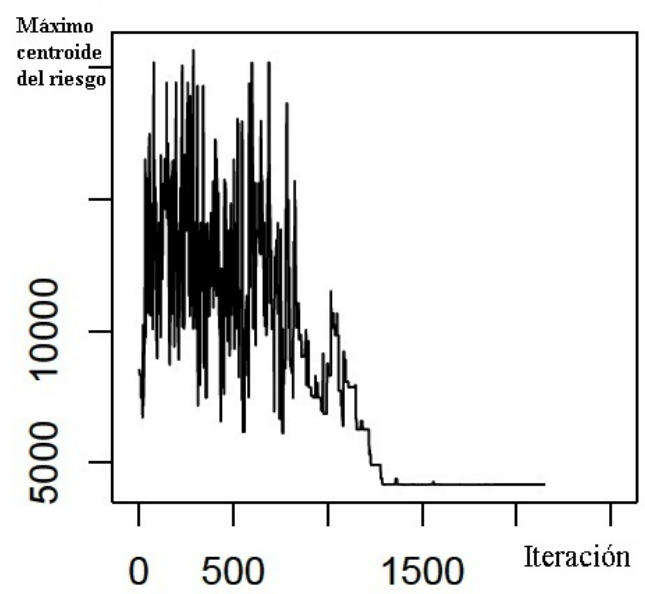

Figura 7.4.2: Trayectoria del máximo centroide del riesgo en la búsqueda de la solución óptima

propuestas se muestra en la Tabla 7.4.7.

Tabla 7.4.6: Solución

\begin{tabular}{|c|c|c|c|c|}
\hline & S. transmisión & & S. preventivas & S. paliativas \\
\hline arco & paquete & amenaza & paquete & paquete \\
\hline$d\left(A_{4}, A_{5}\right)$ & $(10100111)$ & $T_{1}^{1}$ & $(0011)$ & $(000011)$ \\
\hline$d\left(A_{3}, A_{5}\right)$ & (10111110) & $T_{1}^{2}$ & $(00)$ & $(110)$ \\
\hline$d\left(A_{2}, A_{5}\right)$ & $(110)$ & $T_{2}^{2}$ & $(00)$ & $(101)$ \\
\hline$d\left(A_{2}, A_{3}\right)$ & (01111) & $T_{1}^{3}$ & $(0010)$ & $(00000)$ \\
\hline$d\left(A_{1}, A_{4}\right)$ & $(0100)$ & $T_{1}^{4}$ & $(0100)$ & $(00000)$ \\
\hline$d\left(A_{1}, A_{2}\right)$ & (0101) & & & \\
\hline
\end{tabular}

Tabla 7.4.7: Riesgo sobre $A_{5}$ obtenido tras la implementación de las salvaguardas propuestas

\begin{tabular}{|c|c|c|c|}
\hline & $\mathrm{C}$ & I & $\mathrm{D}$ \\
\hline$T_{1}^{1}$ & $(16.87,161.72,936.23,3681.49)$ & $(32.70,239.68,1295.59,5197.40)$ & $(25.12,198.56,1576.70,5777.12)$ \\
\hline$T_{1}^{2}$ & $(0,49.58,458.07,1791.16)$ & $(0,29.75,289.73,1397.10)$ & $(0,24.64,352.60,1552.93)$ \\
\hline$T_{2}^{2}$ & $(0,49.58,458.07,1791.16)$ & $(0,29.75,289.73,1397.10)$ & $(76.02,379.33,2074.33,5588.43)$ \\
\hline$T_{1}^{3}$ & $(12.18,110.49,647.25,2465.61)$ & $(21.93,147.32,744.34,2958.74)$ & $(6.82,58.49,487.14,1923.18)$ \\
\hline$T_{1}^{4}$ & $(34.81,245.53,1176.82,3793.25)$ & $(62.67,327.37,1353.35,4551.90)$ & $(19.49,129.98,885.71,2958.74)$ \\
\hline
\end{tabular}




\section{Capítulo 8 UN EJEMPLO ILUSTRATIVO}

\subsection{Introducción}

En este capítulo presentamos un ejemplo tomado de la documentación oficial de la metodología MAGERIT [37]. El enunciado del problema se ha tomado íntegramente de dicha documentación, y para resolverlo utilizaremos la metodología borrosa desarrollada en esta tesis doctoral. El ejemplo analiza el caso de una unidad administrativa que utiliza un sistema de información (SI) para el desarrollo de sus tareas internas y para la prestación de servicios de atención administrativa a los ciudadanos (administración electrónica).

La unidad que se presenta lleva años tramitando expedientes en el ámbito municipal. En los primeros años lo hacía de forma manual y actualmente por medio de un sistema informático propio. El sistema almacena la información en un archivo central que funciona como memoria histórica, permitiendo recuperar datos y conservar los expedientes cerrados.

Los usuarios pueden realizar sus tramitaciones vía web, usando su NIF y una contraseña personal como identificación, pero también tienen la opción de realizar estos procedimientos de forma presencial a través de un funcionario de la oficina que tiene acceso directo al sistema informático.

El responsable del proyecto de administración electrónica, preocupado por la seguridad del sistema de información y consciente de que un fallo en el servicio conllevaría un serio daño a la imagen de su unidad, argumenta la conveniencia de lanzar un proyecto de análisis y gestión de riesgos sobre el SI.

Se constituye un comité de seguimiento formado por los responsables de los servicios involucrados: atención a usuarios, asesoría jurídica, servicios informáticos y seguridad física, y se mantiene una reunión inicial con la dirección de la unidad administrativa en la que se exponen los puntos principales de un primer análisis realizado por el responsable del 
proyecto de administración electrónica, que queda habilitado como director del proyecto de análisis y gestión de riesgos. En este proyecto participarán dos personas de su equipo junto con una empresa consultora externa contratada a la que se le exige identificar nominalmente a las personas que van a participar y firmar un acuerdo de confidencialidad.

El alcance del proyecto es el servicio de tramitación electrónica, presencial y remoto, y se estudiará la seguridad de los expedientes que se manejan y se almacenan diariamente en el archivo central.

Se analizaron equipos y redes de comunicaciones, dejando en principio fuera del estudio elementos que podrían ser relevantes en un análisis más detallado, como los datos de identificación y autenticación de los usuarios de los sistemas o las instalaciones. En un futuro se prevé lanzar un proyecto de análisis y gestión de riesgos en el SI más detallado que profundice en dichos aspectos. El análisis se limita al ámbito de la administración (local) excluyéndose la evaluación de la seguridad de los servicios subsidiarios que se emplean.

\subsection{Análisis de riesgos}

La fase de análisis de riesgos se inicia con una serie de entrevistas a los responsables designados por el comité de seguimiento con objeto de perfilar y describir correctamente el sistema. Esta información se desglosa en diferentes elementos que se detallan a continuación.

\subsubsection{Servicio de tramitación}

El servicio de tramitación se presta por medio de una aplicación informática desarrollada sobre una base de datos a la que se accede a través de un sistema de identificación local del usuario que controla sus privilegios de acceso. En la tramitación presencial, es el funcionario que está atendiendo al usuario final, quien se identifica frente al sistema. En el caso de la tramitación remota, el usuario final se identifica directamente.

La tramitación incluye una fase de solicitud (entrada de datos) y una fase de respuesta (entrega de datos). La notificación se realiza por correo, certificado en el caso de tramitación presencial, y electrónico en el caso de tramitación electrónica.

El expediente que se abre al iniciar una tramitación se almacena localmente en la 
oficina y se consulta una serie de datos del archivo central de información. Al cierre del expediente, los datos y un informe de las actuaciones realizadas se remiten al archivo central para su custodia, eliminándose la información de los equipos locales.

En todo el proceso de tramitación se usa el correo electrónico como medio interno de comunicación entre el personal y como mecanismo de notificación a los usuarios externos. Como norma, no se debe emplear el correo como transporte de documentos; estos siempre serán servidos por medio de accesos web.

\subsubsection{Servicio de archivo central}

Existe un servicio centralizado de archivo y recuperación de documentos que funciona por medio de una intranet. Los usuarios acceden por medio de una interfaz web local, que se conecta mediante una red privada virtual con el servidor remoto, identificándose por medio de su NIF. Este servicio sólo está disponible para el personal de la unidad y para el empleado virtual que presta el servicio de tramitación remota.

\subsubsection{Equipamiento informático}

La unidad dispone de varios equipos personales que poseen un navegador web, un cliente de correo electrónico sin almacenamiento local de los mensajes y un paquete ofimático estándar (procesador de textos y hoja de cálculo).

No se realizan copias de seguridad de los discos duros de los equipos personales y existe un procedimiento de instalación / actualización que borra el disco local y reinstala el sistema íntegro.

Los equipos no disponen de unidades de memoria extraíble de ningún tipo (disquetes, CD, DVD, USB, etc) y se dispone de un servidor de tamaño medio, de propósito general, dedicado a las tareas de:

- Servidor de ficheros.

- Servidor de mensajería electrónica, con almacenamiento local y acceso vía web.

- Servidor de bases de datos: expedientes en curso e identificación de usuarios.

- Servidor web para la tramitación remota y para la intranet local. 


\subsubsection{Comunicaciones}

Se dispone de una red de área local que cubre las instalaciones de trabajo y la sala de equipos. Está explícitamente prohibida la instalación de módems de acceso remoto y redes inalámbricas, existiendo un procedimiento rutinario de inspección.

Existe una conexión ADSL a Internet contratada a un operador comercial. Sobre esta conexión se prestan múltiples servicios de tramitación remota, de correo electrónico, de acceso a información y como red privada virtual con el archivo central.

La conexión a Internet se realiza a través de un cortafuegos que limita las comunicaciones a nivel de red, permitiendo únicamente el intercambio de correo electrónico con el servidor de correo y el intercambio web con el servidor web.

La red privada virtual se establece al inicio de la jornada, cortándose automáticamente a la hora de cierre. En el establecimiento los equipos terminales se reconocen mutuamente y establecen una clave de sesión para la jornada. No hay intervención de ningún operador local.

Muchos servicios dependen de la conexión a internet, y en ocasiones, se han producido incidencias tales como cortes de servicio debidos a obras municipales y a una deficiente prestación del servicio por parte del proveedor. Por todo ello:

1. Se ha establecido un contrato con el proveedor que establece que en caso de interrupción o mal funcionamiento del servicio, el operador debe abonar unas indemnizaciones pactadas de antemano en proporción al periodo de interrupción.

2. Se ha contratado con otro proveedor un enlace digital (RDSI) de respaldo, enlace que habitualmente no está establecido, pero que se activa automáticamente cuando el enlace ADSL se interrumpe durante más de 10 minutos.

\subsubsection{Seguridad física}

El personal trabaja en las dependencias interiores de la unidad, salvo el personal de atención directa al público que lo hace en las salas externas habilitadas. El acceso a las dependencias internas está limitado a las horas de oficina, quedando cerrado con llave fuera de dicho horario. En horas de oficina hay un control de entrada que identifica a los empleados y registra su hora de entrada y de salida. 


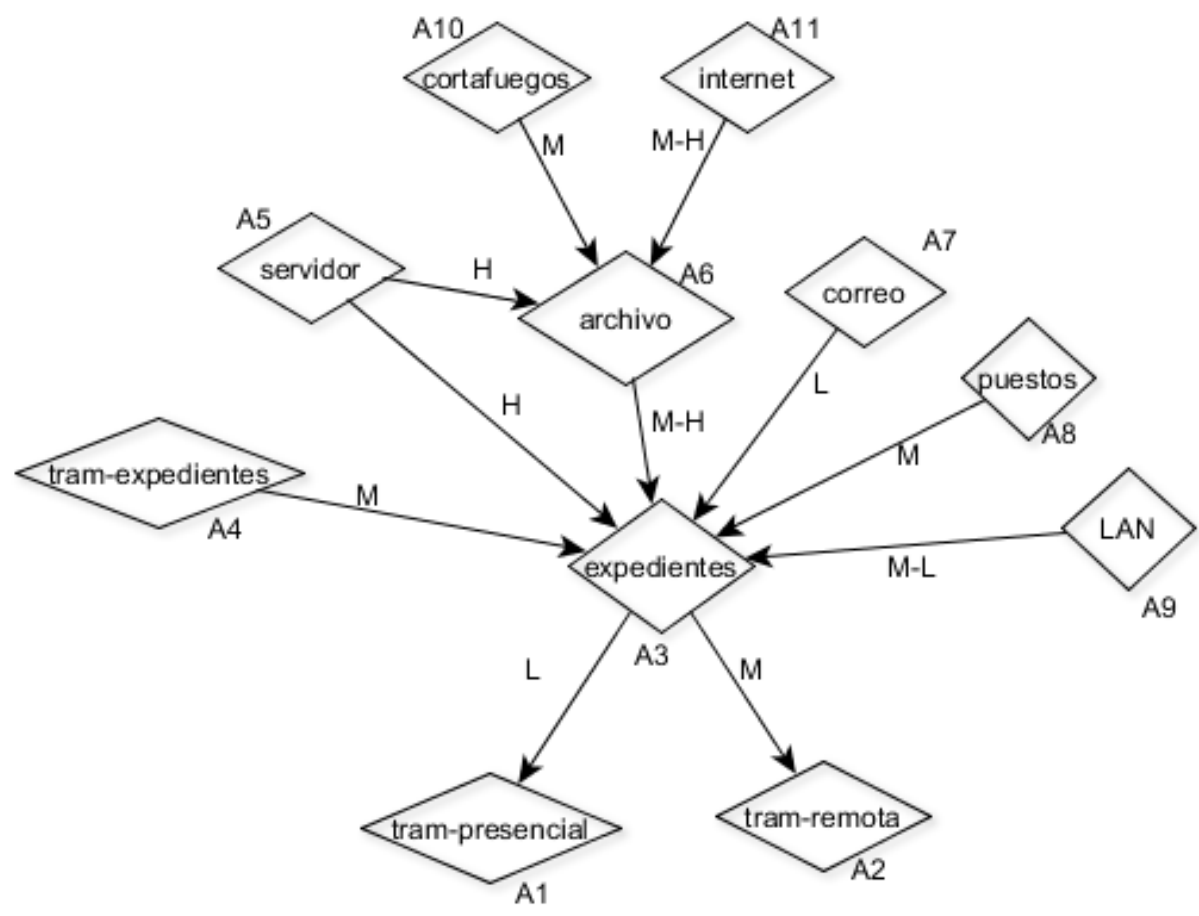

Figura 8.2.1: Dependencias entre activos del SI.

La sala de equipos es una habitación interior que permanece cerrada con llave y cuya responsabilidad recae en el administrador de sistemas. La sala dispone de un sistema de detección y extinción de incendios que se revisa anualmente y dista 50 metros de la canalización de agua más cercana.

Los locales de la unidad ocupan íntegramente la cuarta planta de un edificio de oficinas de 12 plantas. Los controles de acceso son propios de la unidad, no del edificio, que es de uso compartido con otras actividades. Se desconocen las instalaciones de las plantas superior e inferior, que no corresponden a la unidad administrativa.

\subsubsection{Análisis borroso}

A partir de la información establecida en las secciones anteriores se establece el grafo de dependencias de la Figura 8.2.1 y, siguiendo la metodología borrosa propuesta, primero consideramos los términos lingüísticos con sus respectivos números borrosos trapezoidales que se muestran en la Tabla 8.2.1.

A continuación, se realizan entrevistas con los expertos y se establecen los términos que expresan sus grados de influencia entre activos en el SI. Los grados de dependencia 
Tabla 8.2.1: Términos lingüísticos

\begin{tabular}{cc}
\hline Término & Número borroso \\
\hline \hline Muy bajo (VL) & $(0,0,0,0.05)$ \\
\hline Bajo (L) & $(0,0.05,0.15,0.25)$ \\
\hline Medio-Bajo (M-L) & $(0.15,0.25,0.35,0.45)$ \\
\hline Medio (M) & $(0.35,0.45,0.55,0.65)$ \\
\hline Medio-Alto (M-H) & $(0.55,0.65,0.75,0.85)$ \\
\hline Alto(H) & $(0.75,0.85,0.95,1)$ \\
\hline Muy-Alto (VH) & $(0.95,1,1,1)$ \\
\hline
\end{tabular}

Tabla 8.2.2: Grados de dependencias de activos de soporte calculados de forma inmedianta

\begin{tabular}{cccc}
\hline & tram-presencial & expedientes & tram-remota \\
\hline \hline tram-expedientes & $\mathrm{M} \otimes \mathrm{L}$ & $\mathrm{M}$ & $\mathrm{M} \otimes \mathrm{M}$ \\
\hline archivo & $\mathrm{M}-\mathrm{H} \otimes \mathrm{L}$ & $\mathrm{M}-\mathrm{H}$ & $\mathrm{M}-\mathrm{H} \otimes \mathrm{M}$ \\
\hline correo & $\mathrm{L} \otimes \mathrm{L}$ & $\mathrm{L}$ & $\mathrm{L} \otimes \mathrm{M}$ \\
\hline puestos & $\mathrm{M} \otimes \mathrm{L}$ & $\mathrm{M}$ & $\mathrm{M} \otimes \mathrm{M}$ \\
\hline LAN & $\mathrm{M}-\mathrm{L} \otimes \mathrm{L}$ & $\mathrm{M}-\mathrm{L}$ & $\mathrm{M}-\mathrm{L} \otimes \mathrm{M}$ \\
\hline
\end{tabular}

directos aportados por los expertos se muestran sobre los arcos del grafo de la Figura 8.2.1.

Nótese que únicamente los arcos (servidor, archivo) y (servidor, expedientes) tienen un alto grado de dependencia, mientras que los arcos (internet, archivo) y (archivo, expedientes) tienen un grado de influencia asignado medio alto.

Entonces, calculamos los grados de dependencia de los activos de soporte sobre los activos terminales. En primer lugar, calculamos el grado de dependencia para los activos que influyen directamente sobre los activos terminales, los cuales se obtienen de forma inmediata y se muestran en la Tabla 8.2.2.

La Tabla 8.2.3 muestra los las operaciones de las cuales se derivan los grados de dependencia de los activos cortafuegos, internet y servidor con respecto a los activos terminales, mientras que en la Tabla 8.2.4 se muestran los grados de dependencia resultantes de los activos de soporte sobre los activos terminales.

A continuación, los expertos asignan términos lingüísticos para valorar las componente de cada activo terminal.

La metodología MAGERIT utiliza originalmente una escala de números enteros entre 1 y 10 para valorar las componentes de cada activo [37]. Los criterios de asignación se 
Tabla 8.2.3: Grados de dependencia sobre los activos terminales para cortafuegos, internet y servidor

\begin{tabular}{cccc}
\hline & tram-presencial & expedientes & tram-remota \\
\hline \hline cortafuegos & $\mathrm{M} \otimes \mathrm{M}-\mathrm{H} \otimes \mathrm{L}$ & $\mathrm{M} \otimes \mathrm{M}-\mathrm{H}$ & $\mathrm{M} \otimes \mathrm{M}-\mathrm{H} \otimes \mathrm{M}$ \\
\hline internet & $\mathrm{M}-\mathrm{H} \otimes \mathrm{M}-\mathrm{H} \otimes \mathrm{L}$ & $\mathrm{M}-\mathrm{H} \otimes \mathrm{M}-\mathrm{H}$ & $\mathrm{M}-\mathrm{H} \otimes \mathrm{M}-\mathrm{H} \otimes \mathrm{M}$ \\
\hline servidor & {$[\mathrm{H} \oplus(\mathrm{H} \otimes \mathrm{M}-\mathrm{H})] \otimes \mathrm{L}$} & $\mathrm{H} \oplus(\mathrm{H} \otimes \mathrm{M}-\mathrm{H})$ & {$[\mathrm{H} \oplus(\mathrm{H} \otimes \mathrm{M}-\mathrm{H})] \otimes \mathrm{M}$} \\
\hline
\end{tabular}

especifican oficialmente en la propia documentación de la metodología [37, 38, 39]. En esta tesis hemos trasladado los valores anteriores a la escala de términos lingüísticos, de modo que los valores de los tres activos terminales se muestran en la Tabla 8.2.5.

Nótese que las componentes autenticidad y trazabilidad han sido divididas en este ejemplo en sendas nuevas componentes que corresponden a datos y servicios respectivamente. En consecuencia, se considerarán siete componentes por cada activo: confidencialidad (Confid.), integridad (Integr.), autenticidad de los datos (D. Aut.), autenticidad del servicio (S. Aut.), trazabilidad de los datos (D. Tra.), trazabilidad del servicio (S. Tra.) y disponibilidad (Disp.).

Los valores acumulados son calculados para los activos de soporte por medio de la Ecuación 6.2.1, cuyos resultados se muestran en la Tabla 8.2.6. Por ejemplo, el valor acumulado para el activo archivo en cada componente se calcula de la siguiente forma:

$$
\begin{aligned}
& \widetilde{v}_{\operatorname{archivo}_{(1)}}=\left[(\mathrm{M}-\mathrm{H} \otimes \mathrm{L}) \otimes v_{\text {tram-presencial }}^{(1)}++\right. \\
& +\left[\mathrm{M}-\mathrm{H} \otimes v_{\text {expedientes }_{(1)}}\right]+\left[(\mathrm{M}-\mathrm{H} \otimes \mathrm{M}) \otimes v_{\text {tram-remota }_{(1)}}\right] \\
& =(0,302,0,422,0,562,0,722) \\
& \widetilde{v}_{\operatorname{archivo}_{(2)}}=\left[(\mathrm{M}-\mathrm{H} \otimes \mathrm{L}) \otimes v_{\text {tram-presencial }_{(2)}}\right]+ \\
& +\left[\mathrm{M}-\mathrm{H} \otimes v_{\operatorname{expedientes}_{(2)}}\right]+\left[(\mathrm{M}-\mathrm{H} \otimes \mathrm{M}) \otimes v_{\text {tram-remota }_{(2)}}\right] \\
& =(0,192,0,292,0,412,0,552) \\
& \widetilde{v}_{\operatorname{archivo}_{(7)}}=\left[(\mathrm{M}-\mathrm{H} \otimes \mathrm{L}) \otimes v_{\text {tram-presencial }_{(7)}}\right]+ \\
& +\left[\mathrm{M}-\mathrm{H} \otimes v_{\text {expedientes }_{(7)}}\right]+\left[(\mathrm{M}-\mathrm{H} \otimes \mathrm{M}) \otimes v_{\text {tram-remota }_{(7)}}\right] \\
& =(0,28,0,086,0,197,0,352)
\end{aligned}
$$

A continuación, consideremos una amenaza sobre el activo archivo con una degradación dada por $\widetilde{D}=(\mathrm{H}, \mathrm{L}, \mathrm{VL}, \mathrm{M}, \mathrm{M}-\mathrm{H}, \mathrm{VH}, \mathrm{H})$. La Tabla 8.2 .6 muestra las valoraciones en las siete componentes sobre los activos. El impacto de la amenaza sobre dichas componentes 
Tabla 8.2.4: Grados de dependencia resultantes sobre los activos terminales

\begin{tabular}{cccc}
\hline & tram-presencial & expedientes & tram-remota \\
\hline \hline tram-expedientes & $(0,0.022,0.082,0.162)$ & $(0.35,0.45,0.55,0.65)$ & $(0.122,0.202,0.302,0.422)$ \\
\hline archivo & $(0,0.032,0.112,0.212)$ & $(0.55,0.65,0.75,0.85)$ & $(0.192,0.292,0.412,0.552)$ \\
\hline correo & $(0,0.002,0.022,0.062)$ & $(0,0.05,0.15,0.25)$ & $(0,0.022,0.082,0.162)$ \\
\hline puestos & $(0,0.022,0.082,0.162)$ & $(0.35,0.45,0.55,0.65)$ & $(0.122,0.202,0.302,0.422)$ \\
\hline LAN & $(0,0.012,0.052,0.112)$ & $(0.15,0.25,0.35,0.45)$ & $(0.052,0.1120 .192,0.292)$ \\
\hline cortafuegos & $(00.014,0.061,0.138)$ & $(0.192,0.292,0.412,0.552)$ & $(0.067,0.131,0.226,0.359)$ \\
\hline internet & $(0,0.021,0.084,0.18)$ & $(0.302,0.422,0.562,0.722)$ & $(0.105,0.190,0.309,0.469)$ \\
\hline servidor & $(0,0.046,0.147,0.25)$ & $(0.853,0.932,0.985,1)$ & $(0.298,0.419,0.542,0.65)$ \\
\hline
\end{tabular}

Tabla 8.2.5: Valor de los activos terminales

\begin{tabular}{cccc}
\hline & tram-presencial & expedientes & tram-remota \\
\hline \hline confidencialidad & $\mathrm{M}-\mathrm{H}$ \\
\hline integridad & $\mathrm{M}$ & \\
\hline autenticidad de los datos & & $\mathrm{M}$ & \\
\hline autenticidad del servicio & $\mathrm{M}-\mathrm{H}$ & $\mathrm{M}-\mathrm{H}$ \\
\hline trazabilidad de los datos & & $\mathrm{M}$ & \\
\hline trazabilidad del servicio & $\mathrm{M}$ & & $\mathrm{M}$ \\
\hline disponibilidad & $\mathrm{M}$ & $\mathrm{M}-\mathrm{L}$ \\
\hline
\end{tabular}

se calcula multiplicando por el vector anterior de degradación (Tabla 8.2.7).

Nótese que la función de similitud puede ser usada en cualquier paso de la metodología propuesta para derivar un término lingüístico asociado con el número borroso trapezoidal que resulta de los cálculos de valores acumulados, impacto o riesgo. Sin embargo, es conveniente mantener los números borrosos que se van obteniendo en cada paso con objeto de no perder información a lo largo del proceso.

Usando pesos iguales y la distancia del infinito en la función de similitud de Vicente et al. [62] el impacto sobre las componentes del activo archivo es (M, M-L, VL, L, M-L, M-L, L).

A continuación, suponemos que los expertos asignan una frecuencia alta a la amenaza. Entonces podemos calcular el riesgo mediante la Ecuación 6.2.3, de la que se obtienen los valores de la Tabla 8.2.7. También podemos calcular el impacto y el riesgo sobre los activos que influyen sobre el activo (archivo), es decir, sobre servidor, cortafuegos e internet (Tabla 8.2.7).

Mediante la función de similitud podemos identificar el riesgo sobre el activo archivo en la escala borrosa inicial, que es (M, L, VL, L, M-L, M-L, L). Finalmente, la Tabla 
8.2.8 muestra los términos lingüísticos asociados con el riesgo del activo atacado y sobre aquéllos que influyen sobre éste.

\subsection{Selección de salvaguardas}

A continuación, consideramos el conjunto de salvaguardas de transmisión de fallos entre los activos de soporte que se muestran en la Tabla 8.3.1, y el umbral borroso $\widetilde{U}=$ $(0,0,0.05,0.1)$ bajo el cual el grado de dependencia entre los activos de soporte y los activos terminales es aceptable, y sea $\alpha=0.95$. Entonces, ejecutamos el algoritmo de programación dinámica como sigue:

Etapa 0: $L_{0}=\left\{\right.$ tram-presencial $\left(A_{1}\right)$, tram-remota $\left(A_{2}\right)$, expedientes $\left.\left(A_{3}\right)\right\}$ que son los activos terminales del sistema. Identificamos salvaguardas para reducir el grado de dependencia de $A_{3}$ sobre $A_{1}$ y $A_{2}$ a un nivel aceptable, $S\left(\widetilde{D D}\left(A_{3}, A_{1}\right), \widetilde{U}\right) \geq 0.95$ y $S\left(\widetilde{D D}\left(A_{3}, A_{2}\right), \widetilde{U}\right) \geq 0.95$, respectivamente, de modo que se minimice el coste.

Con respecto al activo $A_{3}$, la solución está representada por el vector $x^{3,1}=\left(x_{1}^{3,1}, \ldots, x_{8}^{3,1}\right)$, (Tabla 8.3.1) donde $x_{p}^{3,1}=1$ si la salvaguarda $S_{p}^{3,1}$ es seleccionada. Para el activo $A_{2}$ se obtiene la solución $x^{3,2}=\left(x_{1}^{3,2}, \ldots, x_{10}^{3,2}\right)$. Ambos conjuntos de salvaguardas son seleccionados de forma independiente tras resolver los siguientes problemas de optimización:

$$
\begin{array}{cc}
\text { Minimizar } \quad c_{1}^{3,1} x_{1}^{3,1}+\ldots+c_{8}^{3,1} x_{8}^{3,1} & \text { Minimizar } c_{1}^{3,2} x_{1}^{3,2}+\ldots+c_{10}^{3,2} x_{10}^{3,2} \\
\text { s.a } & \text { s.a } \\
\qquad\left(\widetilde{D D}\left(A_{3}, A_{1}\right), \widetilde{U}\right) \geq 0.95 & \quad S\left(\widetilde{D D}\left(A_{3}, A_{2}\right), \widetilde{U}\right) \geq 0.95 \\
\quad x_{p}^{3,1} \in\{0,1\}, p=1, \ldots, 8 & x_{q}^{3,2} \in\{0,1\}, q=1, \ldots, 10
\end{array}
$$

La solución óptima es $x^{3,1^{*}}=(0,0,1,0,0,0,0,1)$ y $x^{3,2^{*}}=(0,0,1,0,0,0,1,1,0,0)$, con costes 220 y 646, respectivamente (Tabla 8.3.2) y se obtiene como resultado de aplicar recocido simulado, tal y como se ha indicado en capítulos anteriores.

Etapa 1: $L_{1}=\left\{\right.$ tram-expedientes $\left(A_{4}\right)$, archivo $\left(A_{6}\right)$, correo $\left(A_{7}\right)$, puestos $\left(A_{8}\right)$, LAN $\left.\left(A_{9}\right)\right\}$. Seleccionamos salvaguardas para reducir los grados de dependencia directos entre los activos de $L_{1}$ y los activos terminales a un nivel aceptable, $S\left(\widetilde{D D}\left(A_{i}, A_{1}\right), \widetilde{U}\right) \geq 0.95$, $S\left(\widetilde{D D}\left(A_{i}, A_{2}\right), \widetilde{U}\right) \geq 0.95$ y $S\left(\widetilde{D D}\left(A_{i}, A_{3}\right), \widetilde{U}\right) \geq 0.95$, con $A_{i} \in L_{1}$, minimizando los 
Tabla 8.2.6: Valor acumulado por los activos de soporte

\begin{tabular}{|c|c|c|}
\hline Comp. & tram-expedientes & archivo \\
\hline Confid. & 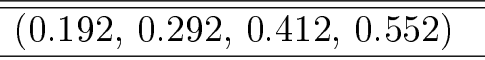 & $(0.302,0.422,0.562,0.722)$ \\
\hline Integr. & $(0.122,0.202,0.302,0.422)$ & $(0.192,0.292,0.412,0.552)$ \\
\hline D. Aut. & $(0.122,0.202,0.302,0.422)$ & $(0.192,0.292,0.412,0.552)$ \\
\hline S. Aut. & $(0.067,0.144,0.274,0.447)$ & $(0.105,0.207,0.367,0.565)$ \\
\hline D. Tra. & $(0.122,0.202,0.302,0.422)$ & $(0.192,0.292,0.412,0.552)$ \\
\hline S. Tra. & $(0.042,0.100,0.204,0.351)$ & $(0.067,0.144,0.274,0.447)$ \\
\hline Disp. & $(0.018,0.060,0.146,0.275)$ & $(0.028,0.086,0.197,0.352)$ \\
\hline Comp. & correo & puestos \\
\hline Confid. & $(0.0,0.032,0.112,0.212)$ & $(0.192,0.292,0.412,0.552)$ \\
\hline Integr. & $(0.0,0.022,0.082,0.162)$ & $(0.122,0.202,0.302,0.422)$ \\
\hline D. Aut. & $(0.0,0.022,0.082,0.162)$ & $(0.122,0.202,0.302,0.422)$ \\
\hline S. Aut. & $(0.0,0.016,0.077,0.183)$ & $(0.067,0.144,0.2740 .447)$ \\
\hline D. Tra. & $(0.0,0.022,0.082,0.162)$ & $(0.122,0.202,0.302,0.422)$ \\
\hline S. Tra. & $(0.0,0.011,0.057,0.141$ & $(0.042,0.100,0.204,0.351)$ \\
\hline Disp. & $(0.0,0.006,0.040,0.1110$ & $(0.018,0.060,0.146,0.275)$ \\
\hline Comp. & & cortafuegos \\
\hline Confid. & 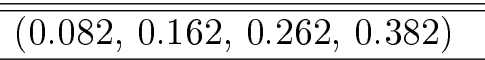 & 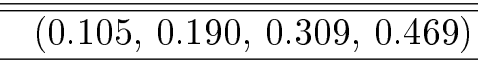 \\
\hline Integr. & $(0.052,0.112,0.192,0.292)$ & $(0.067,0.131,0.226,0.359)$ \\
\hline D. Aut. & $(0.052,0.112,0.192,0.292)$ & $(0.067,0.131,0.226,0.359)$ \\
\hline S. Aut. & $(0.028,0.080,0.178,0.320)$ & $(0.037,0.094,0.208,0.386)$ \\
\hline D. Tra. & $(0.052,0.112,0.192,0.292)$ & $(0.067,0.131,0.226,0.359)$ \\
\hline S. Tra. & $(0.018,0.055,0.131,0.249)$ & $(0.023,0.065,0.154,0.302)$ \\
\hline Disp. & $(0.007,0.033,0.094,0.195)$ & $(0.010,0.039,0.110,0.236)$ \\
\hline Comp. & internet & servidor \\
\hline Confid. & $(0.166,0.274,0.625,0.614)$ & 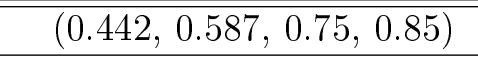 \\
\hline Integr. & $(0.105,0.190,0.309,0.469)$ & $(0.281,0.406,0.55,0.65)$ \\
\hline D. Aut. & $(0.105,0.190,0.309,0.469)$ & $(0.281,0.406,0.550 .65)$ \\
\hline S. Aut. & $(0.058,0.135,0.280,0.491)$ & $(0.132,0.264,0.478,0.647)$ \\
\hline D. Tra. & $(0.105,0.190,0.309,0.469)$ & $(0.281,0.406,0.55,0.65)$ \\
\hline S. Tra. & $(0.037,0.094,0.208,0.386)$ & $(0.084,0.183,0.360,0.516)$ \\
\hline Disp. & $(0.015,0.056,0.149,0.303)$ & $(0.036,0.101,0.259,0.407)$ \\
\hline
\end{tabular}


Tabla 8.2.7: Impacto y riesgo sobre los activos archivo, servidor, cortafuegos e internet

\begin{tabular}{ccc}
\hline & & \\
Comp. & Impacto $($ archivo $)$ & Riesgo $($ archivo $)$ \\
\hline \hline Confid. & $(0.227,0.359,0.534,0.722)$ & $(0.170,0.305,0.508,0.722)$ \\
\hline Integr. & $(0,0.015,0.062,0.138)$ & $(0,0.012,0.059,0.138)$ \\
\hline D. Aut. & $(0,0,0,0.028)$ & $(0,0,0,0.028)$ \\
\hline S. Aut. & $(0.037,0.093,0.202,0.367)$ & $(0.028,0.079,0.192,0.367)$ \\
\hline D. Tra. & $(0.106,0.190,0.309,0.469)$ & $(0.079,0.162,0.294,0.470)$ \\
\hline S. Tra. & $(0.064,0.144,0.275,0.448)$ & $(0.048,0.123,0.261,0.448)$ \\
\hline Disp. & $(0.022,0.074,0.187,0.352)$ & $(0.016,0.063,0.178,0.352)$ \\
\hline & & \\
Comp. & Impacto $($ servidor $)$ & Riesgo $($ servidor $)$ \\
\hline \hline Confid. & $(0.283,0.465,0.702,0.85)$ & $(0.212,0.396,0.667,0.85)$ \\
\hline Integr. & $(0,0.019,0.081,0.162)$ & $(0,0.016,0.077,0.162)$ \\
\hline D. Aut. & $(0,0,0,0.0325)$ & $(0,0,0,0.032)$ \\
\hline S. Aut. & $(0.040,0.111,0.259,0.421)$ & $(0.030,0.094,0.246,0.421)$ \\
\hline D. Tra. & $(0.132,0.247,0.407,0.552)$ & $(0.099,0.201,0.386,0.552)$ \\
\hline S. Tra. & $(0.068,0.171,0.355,0.516)$ & $(0.051,0.145,0.337,0.516)$ \\
\hline Disp. & $(0.023,0.081,0.243,0.407)$ & $(0.017,0.069,0.230,0.407)$ \\
\hline & & \\
Comp. & Impacto $($ cortafuegos $)$ & Riesgo $($ cortafuegos $)$ \\
\hline \hline Confid. & $(0.028,0.073,0.1612,0.305)$ & $(0.021,0.062,0.154,0.305)$ \\
\hline Integr. & $(0,0.002,0.019,0.058)$ & $(0,0.002,0.018,0.058)$ \\
\hline D. Aut. & $(0,0,0,0.012)$ & $(0,0,0,0.012)$ \\
\hline S. Aut. & $(0.005,0.019,0.063,0.163)$ & $(0.003,0.016,0.06,0.163)$ \\
\hline D. Tra. & $(0.013,0.038,0.094,0.198)$ & $(0.010,0.033,0.089,0.198)$ \\
\hline S. Tra. & $(0.008,0.029,0.085,0.196)$ & $(0.006,0.025,0.081,0.196)$ \\
\hline Disp. & $(0.003,0.015,0.058,0.154)$ & $(0.002,0.013,0.055,0.154)$ \\
\hline Comp. & Impacto $($ internet $)$ & Riesgo (internet $)$ \\
\hline \hline Confid. & $(0.069,0.152,0.301,0.522)$ & $(0.051,0.129,0.286,0.522)$ \\
\hline Integr. & $(0,0.006,0.035,0.1)$ & $(0,0.005,0.033,0.01)$ \\
\hline D. Aut. & $(0,0,0,0.02)$ & $(0,0,0,0.02)$ \\
\hline S. Aut. & $(0.011,0.04,0.116,0.272)$ & $(0.008,0.034,0.11,0.272)$ \\
\hline D. Tra. & $(0.032,0.080,0.174,0.339)$ & $(0.024,0.068,0.165,0.339)$ \\
\hline S. Tra. & $(0.019,0.061,0.156,0.329)$ & $(0.015,0.052,0.149,0.329)$ \\
\hline Disp. & $(0.007,0.031,0.107,0.258)$ & $(0.005,0.027,0.101,0.258)$ \\
\hline & & \\
\hline
\end{tabular}


Tabla 8.2.8: Términos lingüísticos para el riesgo sobre cada activo

\begin{tabular}{ccccc}
\hline Component & archivo & cortafuegos & internet & servidor \\
\hline \hline confidencialidad & $\mathrm{M}$ & $\mathrm{L}$ & $\mathrm{M}-\mathrm{L}$ & $\mathrm{M}$ \\
\hline integridad & $\mathrm{L}$ & $\mathrm{VL}$ & $\mathrm{VL}$ & $\mathrm{L}$ \\
\hline autenticidad de los datos & $\mathrm{VL}$ & $\mathrm{VL}$ & $\mathrm{VL}$ & $\mathrm{VL}$ \\
\hline autenticidad del servicio & $\mathrm{L}$ & $\mathrm{L}$ & $\mathrm{L}$ & $\mathrm{L}$ \\
\hline trazabilidad de los datos & $\mathrm{M}-\mathrm{L}$ & $\mathrm{L}$ & $\mathrm{L}$ & $\mathrm{M}-\mathrm{L}$ \\
\hline trazabilidad del servicio & $\mathrm{M}-\mathrm{L}$ & $\mathrm{L}$ & $\mathrm{L}$ & $\mathrm{M}-\mathrm{L}$ \\
\hline disponibilidad & $\mathrm{L}$ & $\mathrm{L}$ & $\mathrm{L}$ & $\mathrm{L}$ \\
\hline
\end{tabular}

costes.

Nótese que $\widetilde{D D}\left(A_{4}, A_{1}\right)=\widetilde{d d}\left(A_{4}, A_{3}\right) \otimes \widetilde{D D}\left(A_{3}, A_{1}\right)$ y $\widetilde{D D}\left(A_{4}, A_{2}\right)=\widetilde{d d}\left(A_{4}, A_{3}\right) \otimes$ $\widetilde{D D}\left(A_{3}, A_{2}\right)$, y el valor de $\widetilde{D D}\left(A_{3}, A_{1}\right)$, así como $\widetilde{D D}\left(A_{3}, A_{2}\right)$, ya han sido calculados en la etapa anterior, y análogamente con el resto de activos de $L_{1}$.

Para seleccionar el conjunto de salvaguardas sobre $L_{1}$ debemos resolver cinco problemas de optimización, por ejemplo, para tram-expedientes resolvemos el problema:

$$
\begin{array}{r}
\text { Minimizar } \quad c_{1}^{4,3} x_{1}^{4,3}+\ldots+c_{8}^{4,3} x_{8}^{4,3} \\
\text { s.a } \\
S\left(\widetilde{D D}\left(A_{4}, A_{1}\right), \widetilde{U}\right) \geq 0.95 \\
S\left(\widetilde{D D}\left(A_{4}, A_{2}\right), \widetilde{U}\right) \geq 0.95 \\
S\left(\widetilde{D D}\left(A_{4}, A_{3}\right), \widetilde{U}\right) \geq 0.95 \\
x_{p}^{4,3} \in\{0,1\}, p=1, \ldots, 8
\end{array}
$$

La solución óptima a partir del recocido simulado es $x^{4,3^{*}}=(0,1,1,1,0,0,0,0)$, con un coste asociado de 623 . El resto de soluciones con sus respectivos costes se muestran en la Tabla 8.3.2.

Etapa 2: $L_{2}=\left\{\right.$ servidor $\left(A_{5}\right)$, cortafuegos $\left(A_{10}\right)$, internet $\left.\left(A_{11}\right)\right\}$. Identificamos un conjunto de salvaguardas de coste mínimo que mantenga los grados de dependencia de los activos $L_{2}$ sobre los activos terminales por debajo del nivel aceptable.

El grado de dependencia del activo servidor $\left(A_{5}\right)$ sobre los activos terminales puede 
Tabla 8.3.1: Salvaguardas disponibles

\begin{tabular}{cc}
\hline Activo & Salvaguarda $\left(\right.$ etiqueta $\left(S_{p}^{u, v}\right)$, efecto $\left(e_{p}^{u, v}\right)$, coste $\left.\left(c_{p}^{u, v}\right)\right)$ \\
\hline \hline expedientes & $S^{3,1}:\left\{\left(S_{1}^{3,1}, \mathrm{M}, 250\right),\left(S_{2}^{3,1}, \mathrm{~L}, 120\right),\left(S_{3}^{3,1}, \mathrm{~L}, 100\right),\left(S_{4}^{3,1}, \mathrm{M}-\mathrm{L}, 179\right)\right.$, \\
& $\left.\left(S_{5}^{3,1}, \mathrm{M}, 225\right),\left(S_{6}^{3,1}, \mathrm{M}-\mathrm{L}, 160\right),\left(S_{7}^{3,1}, \mathrm{~L}, 100\right),\left(S_{8}^{3,1}, \mathrm{M}-\mathrm{L}, 120\right)\right\}$ skip skip \\
& $S^{3,2}:\left\{\left(S_{1}^{3,2}, \mathrm{~L}, 100\right),\left(S_{2}^{3,2}, \mathrm{M}-\mathrm{L}, 127\right),\left(S_{3}^{3,2}, \mathrm{M}, 234\right),\left(S_{4}^{3,2}, \mathrm{~L}, 180\right),\left(S_{5}^{3,2}, \mathrm{~L}, 147\right)\right.$, \\
& $\left.\left(S_{6}^{3,2}, \mathrm{~L}, 127\right),\left(S_{7}^{3,2}, \mathrm{M}, 234\right),\left(S_{8}^{3,2}, \mathrm{M}, 178\right),\left(S_{9}^{3,2}, \mathrm{~L}, 220\right),\left(S_{10}^{3,2}, \mathrm{M}-\mathrm{L}, 170\right)\right\}$ \\
\hline tram-exp. & $S^{4,3}:\left\{\left(S_{1}^{4,3}, \mathrm{M}-\mathrm{L}, 196\right),\left(S_{2}^{4,3}, \mathrm{~L}, 108\right),\left(S_{3}^{4,3}, \mathrm{M}, 205\right),\left(S_{4}^{4,3}, \mathrm{M}-\mathrm{H}, 310\right)\right.$, \\
& $\left.\left(S_{5}^{4,3}, \mathrm{M}, 245\right),\left(S_{6}^{4,3}, \mathrm{~L}, 169\right),\left(S_{7}^{4,3}, \mathrm{M}-\mathrm{L}, 208\right),\left(S_{8}^{4,3}, \mathrm{M}, 254\right)\right\}$ \\
\hline cortafuegos & $S^{10,6}:\left\{\left(S_{1}^{10,6}, \mathrm{M}, 234\right),\left(S_{2}^{10,6}, \mathrm{M}, 267\right),\left(S_{3}^{10,6}, \mathrm{M}, 215\right),\left(S_{4}^{10,6}, \mathrm{M}-\mathrm{H}, 280\right)\right.$, \\
& $\left.\left(S_{5}^{10,6}, \mathrm{M}-\mathrm{L}, 200\right),\left(S_{6}^{10,6}, \mathrm{M}-\mathrm{H}, 295\right),\left(S_{7}^{10,6}, \mathrm{~L}, 167\right),\left(S_{8}^{10,6}, \mathrm{M}, 203\right)\right\}$ \\
\hline internet & $S^{11,6}:\left\{\left(S_{1}^{11,6}, \mathrm{M}-\mathrm{H}, 302\right),\left(S_{2}^{11,6}, \mathrm{M}-\mathrm{L}, 129\right),\left(S_{3}^{11,6}, \mathrm{M}, 235\right)\right.$, \\
& $\left.\left(S_{4}^{11,6}, \mathrm{M}, 256\right),\left(S_{5}^{11,6}, \mathrm{M}, 231\right),\left(S_{6}^{11,6}, \mathrm{~L}, 178\right),\left(S_{7}^{11,6}, \mathrm{M}-\mathrm{H}, 289\right)\right\}$ \\
\hline servidor & $S^{5,3}:\left\{\left(S_{1}^{5,3}, \mathrm{M}, 207\right),\left(S_{2}^{5,3}, \mathrm{~L}, 109\right),\left(S_{3}^{5,3}, \mathrm{M}-\mathrm{H}, 245\right),\left(S_{4}^{5,3}, \mathrm{M}, 267\right),\left(S_{5}^{5,3}, \mathrm{M}-\mathrm{L}, 102\right)\right\}$ \\
& $S^{5,6}:\left\{\left(S_{1}^{5,6}, \mathrm{M}, 238\right),\left(S_{2}^{5,6}, \mathrm{~L}, 134\right),\left(S_{3}^{5,6}, \mathrm{M}-\mathrm{L}, 256\right),\left(S_{4}^{5,6}, \mathrm{~L}, 111\right),\left(S_{5}^{5,6}, \mathrm{M}, 208\right)\right\}$ \\
\hline archivo & $S^{6,3}:\left\{\left(S_{1}^{6,3}, \mathrm{M}, 248\right),\left(S_{2}^{6,3}, \mathrm{M}, 224\right),\left(S_{3}^{6,3}, \mathrm{M}, 200\right)\right.$, \\
& $\left.\left(S_{4}^{6,3}, \mathrm{M}-\mathrm{L}, 167\right),\left(S_{5}^{6,3}, \mathrm{~L}, 110\right),\left(S_{6}^{6,3}, \mathrm{M}-\mathrm{H}, 256\right)\right\}$ \\
\hline puestos & $S^{8,3}:\left\{\left(S_{1}^{8,3}, \mathrm{M}, 257\right),\left(S_{2}^{8,3}, \mathrm{M}, 234\right),\left(S_{3}^{8,3}, \mathrm{~L}, 189\right)\right.$, \\
& $\left.\left(S_{4}^{8,3}, \mathrm{M}, 236\right),\left(S_{5}^{8,3}, \mathrm{M}, 204\right),\left(S_{6}^{8,3}, \mathrm{~L}, 104\right)\right\}$ \\
\hline correo & $S^{9,3}:\left\{\left(S_{1}^{9,3}, \mathrm{~L}, 110\right),\left(S_{2}^{9,3}, \mathrm{~L}, 124\right),\left(S_{3}^{9,3}, \mathrm{M}-\mathrm{L}, 143\right)\right.$, \\
& $\left.\left(S_{4}^{9,3}, \mathrm{M}, 206\right),\left(S_{5}^{9,3}, \mathrm{M}, 237\right),\left(S_{6}^{9,3}, \mathrm{~L}, 170\right)\right\}$ \\
\hline LAN & $S^{7,3}:\left\{\left(S_{1}^{7,3}, \mathrm{M}, 234\right),\left(S_{2}^{7,3}, \mathrm{~L}, 201\right),\left(S_{3}^{7,3}, \mathrm{M}, 245\right)\right.$, \\
& $\left.\left(S_{4}^{7,3}, \mathrm{~L}, 178\right),\left(S_{5}^{7,3}, \mathrm{M}, 205\right),\left(S_{6}^{7,3}, \mathrm{M}, 200\right)\right\}$ \\
\hline
\end{tabular}

Tabla 8.3.2: Selección óptima de salvaguardas y costes asociados

\begin{tabular}{|c|c|c|c|}
\hline Activo & Solución óptima & Coste & Etapa \\
\hline \multirow[t]{2}{*}{ expedientes } & $x^{3,2^{*}}=(0,0,1,0,0,0,1,1,0,0)$ & 646 & 0 \\
\hline & $x^{3,1^{*}}=(0,0,1,0,0,0,0,1)$ & 220 & 0 \\
\hline tram-expedientes & $x^{4,3^{*}}=(0,1,1,1,0,0,0,0)$ & 623 & 1 \\
\hline archivo & $x^{6,3^{*}}=(0,1,1,0,0,1)$ & 680 & 1 \\
\hline correo & $x^{7,3^{*}}=(0,0,0,1,0,0)$ & 206 & 1 \\
\hline puestos & $x^{8,3^{*}}=(0,1,0,1,1,0)$ & 674 & 1 \\
\hline$L A N$ & $x^{9,3^{*}}=(0,0,0,1,1,1)$ & 583 & 1 \\
\hline \multirow[t]{2}{*}{ servidor } & $x^{5,3^{*}}=(1,0,1,1,1)$ & 1267 & 2 \\
\hline & $x^{5,6^{*}}=(1,0,0,0,1)$ & & 2 \\
\hline cortafuegos & $x^{10,6^{*}}=(0,0,0,0,0,0,0,0)$ & 0 & 2 \\
\hline internet & $x^{11,6^{*}}=(0,0,0,0,0,0,0)$ & 0 & 2 \\
\hline
\end{tabular}


Tabla 8.3.3: Grado de dependencia después de la implementación de salvaguardas

\begin{tabular}{cccc}
\hline Activo & tram-presencia & expedientes & tram-remota \\
\hline \hline expedientes & $(0,0.002,0.08,0.16)$ & 1 & $(0,0.04,0.09,0.17)$ \\
\hline tram-exp. & $(0,0.01,0.05,0.13)$ & $(0.01,0.04,0.10,0.19)$ & $(0,0.01,0.05,0.11)$ \\
\hline archivo & $(0,0.01,0.05,0.13)$ & $(0.01,0.04,0.10,0.19)$ & $(0,0.01,0.05,0.11)$ \\
\hline correo & $(0,0,0.01,0.05)$ & $(0,0.02,0.08,0.16)$ & $(0,0,0.01,0.04)$ \\
\hline puestos & $(0,0.01,0.05,0.13)$ & $(0.01,0.04,0.09,0.17)$ & $(0,0.01,0.05,0.11)$ \\
\hline LAN & $(0,0,0.03,0.09)$ & $(0.01,0.04,0.10,0.19)$ & $(0,0.01,0.03,0.08)$ \\
\hline servidor & $(0,0,0,0.03)$ & $(0,0.03,0.100 .22)$ & $(0,0,0,0.03)$ \\
\hline cortafuegos & $(0,0,0.02,0.08)$ & $(0,0.01,0.05,0.12)$ & $(0,0,0.02,0.07)$ \\
\hline internet & $(0,0,0.03,0.11)$ & $(0,0.02,0.07,0.16)$ & $(0,0,0.03,0.09)$ \\
\hline
\end{tabular}

se calcula como sigue:

$$
\begin{aligned}
& \widetilde{D D}\left(A_{5}, A_{1}\right)=\left[\left(\widetilde{d d}\left(A_{5}, A_{6}\right) \otimes \widetilde{D D}\left(A_{6}, A_{3}\right)\right) \oplus \widetilde{d d}\left(A_{5}, A_{3}\right)\right] \otimes \widetilde{D D}\left(A_{3}, A_{1}\right), \\
& \widetilde{D D}\left(A_{5}, A_{2}\right)=\left[\left(\widetilde{d d}\left(A_{5}, A_{6}\right) \otimes \widetilde{D D}\left(A_{6}, A_{3}\right)\right) \oplus \widetilde{d d}\left(A_{5}, A_{3}\right)\right] \otimes \widetilde{D D}\left(A_{3}, A_{2}\right), \\
& \widetilde{D D}\left(A_{5}, A_{3}\right)=\left(\widetilde{d d}\left(A_{5}, A_{6}\right) \otimes \widetilde{D D}\left(A_{6}, A_{3}\right)\right) \oplus \widetilde{d d}\left(A_{5}, A_{3}\right),
\end{aligned}
$$

$\widetilde{D D}\left(A_{3}, A_{1}\right)$ y $\widetilde{D D}\left(A_{3}, A_{2}\right)$ fueron calculados en la Etapa 0 , mientras que $\widetilde{D D}\left(A_{6}, A_{3}\right)$ fue calculado en la Etapa 1.

El problema de optimización correspondiente al activo servidor $\left(A_{5}\right)$ es

$$
\begin{aligned}
& \text { Minimizar } \quad c_{1}^{5,3} x_{1}^{5,3}+\ldots+c_{5}^{5,3} x_{5}^{5,3}+c_{1}^{5,6} x_{1}^{5,6}+\ldots+c_{5}^{5,6} x_{5}^{5,6} \\
& \text { s.a } \\
& S\left(\widetilde{D D}\left(A_{5}, A_{1}\right), \widetilde{U}\right) \geq 0.95 \\
& S\left(\widetilde{D D}\left(A_{5}, A_{2}\right), \widetilde{U}\right) \geq 0.95 \\
& S\left(\widetilde{D D}\left(A_{5}, A_{3}\right), \widetilde{U}\right) \geq 0.95 \\
& x_{p}^{5,3}, x_{p}^{5,6} \in\{0,1\}, p=1, \ldots, 5
\end{aligned}
$$

y las soluciones óptimas que resultan utilizando recocido simulado son $x^{5,3^{*}}=(1,0,1,1,1)$ y $x^{5,6^{*}}=(1,0,0,0,1)$ con un coste asociado de 1267 (Tabla 8.3.2).

La Tabla 8.3.2 muestra las soluciones óptimas obtenidas en todo el proceso, mientras que la Tabla 8.3.3 muestra el grado de dependencia de los activos de soporte sobre los activos terminales después de implementar las salvaguardas seleccionadas.

Nótese que no se han seleccionado salvaguardas sobre los activos cortafuegos $\left(A_{10}\right)$ e internet $\left(A_{11}\right)$ (Tabla 8.3.3). Este hecho se debe a que la implementación de salvaguardas óptimas para el activo archivo en la primera etapa reduce el grado de dependencia de 
dichos activos sobre los activos terminales (véase la Tabla 8.3.3, fila 3). En consecuencia, el grado de dependencia de los activos cortafuegos $\left(A_{10}\right)$ y internet $\left(A_{11}\right)$, que únicamente están conectados al activo archivo, es suficientemente bajo, de modo que no se requiere implementar nuevas salvaguardas sobre dichos activos.

Si ahora consideramos un umbral borroso $\widetilde{U}=(0,0,0.075,0.15)$ como nivel de aceptación de probabilidades de transmisión de fallos, entonces los costes asociados al conjunto óptimo de salvaguardas se reduce a 4443, como cabría esperar con un umbral más débil.

La Tabla 8.3.4 muestra el resultado completo para este nuevo umbral.

Tabla 8.3.4: Salvaguardas y costes para el nuevo umbral

\begin{tabular}{cccc}
\hline Activo & Solución óptima & Coste & Etapa \\
\hline \hline expedientes & $x^{3,2^{*}}=(\mathbf{1}, \mathbf{1}, 1,0,0,0, \mathbf{0}, 1,0,0)$ & $\mathbf{6 3 9}$ & 0 \\
& $x^{31^{*}}=(0,0, \mathbf{0}, 0,0,0,0,1)$ & $\mathbf{1 2 0}$ & 0 \\
\hline tram-expedientes & $x^{4,3^{*}}=(0, \mathbf{0}, 1,1,0,0,0,0)$ & $\mathbf{5 1 5}$ & 1 \\
\hline archivo & $x^{6,3^{*}}=(0,1,1,0,0,1)$ & 680 & 1 \\
\hline correo & $x^{7,3^{*}}=(0,0, \mathbf{1}, \mathbf{0}, 0,0)$ & $\mathbf{1 4 3}$ & 1 \\
\hline puestos & $x^{8,3^{*}}=(0,1,0,1,1,0)$ & 674 & 1 \\
\hline LAN & $x^{9,3^{*}}=(0,0,0, \mathbf{0}, 1,1)$ & $\mathbf{4 0 5}$ & 1 \\
\hline servidor & $x^{5,3^{*}}=(1,0,1,1,1)$ & 1267 & 2 \\
& $x^{5,6^{*}}=(1,0,0,0,1)$ & & 2 \\
\hline cortafuegos & $x^{10,6^{*}}=(0,0,0,0,0,0,0,0)$ & 0 & 2 \\
\hline internet & $x^{11,6^{*}}=(0,0,0,0,0,0,0)$ & 0 & 2 \\
\hline \multicolumn{4}{c}{}
\end{tabular}


CAPÍTULO 8 


\section{Capítulo 9 CONCLUSIONES Y LINEAS FUTURAS DE INVESTIGACIÓN}

El objetivo principal de esta tesis doctoral era desarrollar un modelo borroso de análisis y gestión de riesgos en los sistemas de información. Este modelo, a medio camino entre los modelos cualitativos y cuantitativos promovidos por las metodologías estatales, basadas a su vez en las normas internacionales de la serie ISO/IEC 27000, posee todas las ventajas de ambos, consistentes en admitir imprecisión en la información aportada por los expertos, al tiempo que permite el tratamiento computacional de la misma.

Los números borrosos trapezoidales son una herramienta interesante para modelar el juicio probabilístico de los expertos en sistemas de ayuda a la decisión cuando la información que se maneja es imprecisa. Estos números extienden a los números reales, de modo que si se posee información precisa sobre algunos elementos del sistema, ésta se puede integrar en el sistema borroso. Además, se puede medir la calidad de la información aportada por medio de la borrosidad y la consistencia de ésta, lo que permite un proceso de filtrado de la información sobre la que se basará el sistema de ayuda a la decisión.

Para la consecución del objetivo principal de la tesis, ha sido necesario alcanzar ciertos objetivos parciales. En primer lugar, se ha propuesto un método interactivo analistaexperto para la extracción de juicios probabilísticos borrosos de expertos sobre un evento cualquiera, evitando sesgos, para lo cual ha sido necesario previamente describir los elementos básicos de la Teoría de la Probabilidad Lingüística, extendiendo a la Teoría Clásica de la Probabilidad.

Por otro lado, se ha propuesto una aritmética adecuada que mejore las aritméticas borrosas usuales, simplificando los algoritmos que vamos a utilizar en el análisis de riesgos en los sistemas de la información, y funciones de similitud que mejoren las funciones se- 
ñaladas en la literatura, aportando propiedades deseables y corrigiendo los inconvenientes encontrados en otras funciones de similitud.

En concreto, se han desarrollado varias funciones de similitud de números borrosos trapezoidales y particularmente se ha construido una nueva función de similitud que establece un consenso entre el resto de funciones de similitud de la literatura. Esta función mejora al resto de funciones por las siguientes razones: tiene mejores propiedades que algunas funciones de la literatura, consideran todos los puntos de vista propuestos por el resto de las funciones de similitud, permite incorporar fácilmente nuevos puntos de vista (parámetros) de modo que puede mejorarse continuamente, y permite evaluar la necesidad de cada parámetro, de modo que podamos prescindir de aquéllos cuyos pesos sean muy reducidos.

Todos los elementos anteriores han sido incorporados en el modelo desarrollado para alcanzar el enfoque borroso del proceso análisis y gestión de riesgos en sistemas de la información. Además, muchos de ellos han supuesto contribuciones originales de calidad dentro del ámbito investigador, como así lo atestiguan las numerosas publicaciones y presentaciones en congresos internacionales derivadas, detalladas más adelante.

Con el modelo resultante, los expertos pueden valorar las probabilidades de fallo, mediante el diálogo metódico con el analista, así como los valores de los activos (en sus cinco componentes), las probabilidades de materialización de las amenazas y la degradación que puede sufrir los activos atacados. A partir de esta información, se obtienen automáticamente los indicadores de impacto y riesgo. Finalmente, se ha presentado un modelo de selección de salvaguardas de reducción de riesgos en los sistemas de información consistente en la reducción de las dependencias entre los activos de soporte y los activos terminales, minimizando costes. Se ha utilizado recocido simulado y programación dinámica en ambiente borroso, de modo que los expertos pueden asignar probabilidades de transmisión de fallos de modo impreciso. Una vez reducidas las probabilidades de transmisión de fallos deben seleccionarse salvaguardas preventivas sobre los activos de soporte.

A continuación, se detallan las publicaciones artículos (3 de ellos incluidos en JCR y uno en el proceso de revisión de una revista del JCR) y capítulos de libro (4) derivadas de la presente tesis doctoral, así como los proceedings (7) de congresos internacionales en los que se ha presentado, lo que muestra la calidad y originalidad del trabajo desarrollado. 
1. Sobre un modelo general de análisis de riesgos, bajo un enfoque borroso, en sistemas de información:

- E. Vicente, A. Mateos, A. Jiménez-Martín (2014), "Risk Analysis in Information Systems: a Fuzzification of the MAGERIT Methodology", Knowledge-Based Systems 66, 1-12. (JCR, Impacto: 3.06, Posición: 15/121 (T1)).

- E. Vicente, A. Mateos y A. Jiménez, "Análisis de Riesgos en los Sistemas de Información", Actas da 8a Conferencia Ibérica de Sistemas e Tecnologías de Informacao (CISTI 2013), ISBN 978-989-96247-9-5, 2013, pp. 557-562.

- E. Vicente, A. Jiménez y A. Mateos, “A Fuzzy Extension of MAGERIT Methodology for Risk Analysis in Information Systems", Proceedings of the IADIS Information Systems Conference (IS 2013), ISBN 978-972-8939-83-0, 2013, pp. $39-46$.

- E. Vicente, A. Jiménez y A. Mateos, "A Fuzzy Approach to Risk Analysis in Information Systems", Proceedings of the 2nd International Conference on Operations Research and Enterprise Systems, ISBN 978-989-8565-40-2, 2013, pp. 130-133.

2. Sobre funciones de similitud de números borrosos trapezoidales:

- E. Vicente-Cestero, A. Jiménez-Martín, A. Mateos (2015), "Similarity Functions for Generalized Trapezoidal Fuzzy Numbers: An Improved Comparative Analysis", Journal of Intelligent Fuzzy Systems 28, 821-833 (JCR, Impacto: 1.812, Posición: 45/123 (T2)).

- E. Vicente, A. Mateos, A. Jiménez-Martín (2014), "Searching for a Consensus Similarity Function for Generalized Trapezoidal Fuzzy Numbers", Decision Making and Soft Computing, R.M. de Moraes, E. E. Kerre, L. dos Santos Machado, J. Lu (eds.), World Scientific Proccedings Series on Computer Engineering and Information Science, Vol. 9, ISBN 978-981-4619-96-7, World Scientific Publishing, pp. $252-257$.

- E. Vicente, A. Mateos y A. Jiménez (2013), “A New Similarity Function for Generalized Trapezoidal Fuzzy Numbers", Artificial Intelligence and Soft Computing, L. Rutkowski, M. Korykowski, R. Scherer, R. Tadeusiewizc, L. Zadeh and J. 
Zurada (eds.), Lecture Notes in Artificial Intelligence 7894, Springer Heidelberg, pp. $400-411$.

3. Sobre un método interactivo analista-decisor de elicitación de probabilidades borrosas:

- E. Vicente, A. Mateos y A. Jiménez-Martín, "A Betting and Lottery-based Method for Fuzzy Probability Elicitation", International Journal of Uncertainty, Fuzziness and Knowledge-Based Systems (en proceso de revisión).

- E. Vicente, A. Mateos y A. Jiménez, "Elicitación de Probabilidades Difusas", Actas del XVII Congreso Español sobre Tecnologías y Lógica Fuzzy, ISBN 97884-15688-76-1, 2014, pp. 33-38.

- E. Vicente, A. Jiménez y A. Mateos, “An interactive method of fuzzy probability elicitation in risk analysis", Proceedings of the 4th International Conference on Risk Analysis and Crisis Response, C. Huang and C. Karahman (eds.), ISBN 978-1-138-00019-3, 2013, CRC Press, 223-228.

4. Sobre la selección de salvaguardas en la gestión del riesgo:

- E. Vicente, A. Mateos, A. Jiménez-Martín (2015), "Fuzzified Risk Management: Selection of Safeguards to Minimize the Maximum Risk", Computer Science and Information Systems 12 (2), 567-585 (JCR, Impacto: 0.477, Posición: 87/104 (T3)).

- E. Vicente, A. Mateos, A. Jiménez-Martín (2015), "A Fuzzy Model for Selecting Safeguards to Reduce Risks in Information Systems", E. Pinson, F. Valente, B. Vitoriano (eds.), Operations Research and Enterprise Systems, Communications in Computer and Information Science 509, ISBN 978-3-319-17508-9, Springer, 63-78.

- E. Vicente, A. Mateos, A. Jiménez (2014), "Selection of Safeguards for Fuzzified Risk Management in Information Systems", A. Rocha, A.M. Correira, F.B Tan, K.A. Stroetmann (eds.), New Perspectives in Information Systems and Technologies, Volume 1, Advances in Intelligent Systems and Computing 275, ISBN 978-3-319-05950-1, Springer, pp. 267-276. 
- A. Jiménez-Martín, E. Vicente y A. Mateos (2015), "Selección de salvaguardas en gestión del riesgo en sistemas de la información: Un enfoque borroso", Revista Ibérica de Sistemas y Tecnologías de la Información 15, ISSN 1646-9895, pp.83100.

- E. Vicente, A. Mateos y A. Jiménez, “A Fuzzy Approach based on Dynamic Programming and Metaheuristics for Selecting Safeguards for Risk Management for Information Systems", Proceedings of the 3rd International Conference on Operations Research and Enterprise Systems, ISBN 978-989-758-017-8, Scitepress, 2014, pp. 35-45.

\subsection{Líneas futuras de investigación}

La mayor crítica recibida por la metodología MAGERIT de análisis y gestión de riesgos en los sistemas de información radica en que se trata de un modelo estático, en el que difícilmente se puede actualizar la información de los expertos, y en el que los parámetros que definen las amenazas (probabilidad y degradación) sobre los activos son constantes. Sin embargo, dichos parámetros pueden cambiar en el tiempo y las condiciones ambientales pueden influir sobre éllos. Por ejemplo, una amenaza de incendio sobre las instalaciones no tendrá las mismas probabilidades de ocurrencia, ni la misma degradación, en todas las estaciones del año.

Es necesario entonces desarrollar modelos que permitan encontrar cuáles son los factores que influyen sobre las amenazas, construyendo bases de datos de incidencias lo más completas posibles, así como determinar en qué cuantía afectan estos factores a los parámetros de las amenazas. En este sentido, serán útiles los modelos de regresión logística borrosa para controlar las probabilidades de materialización de las amenazas, así como métodos de aprendizaje automático que nos permitan inferir la degradación que puede sufrir un activo en cada situación.

Esta información nos permitirá establecer un modelo de vigilancia y alerta temprana que evite la materialización de las amenazas o atenúe la degradación de los activos atacados. De este modo, se podrán identificar una serie de salvaguardas que se tomarán de forma eventual cuando las circunstancias transitorias lo recomienden. 


\section{Bibliografía}

[1] Abbas, A.E., Budescu, David V., Hsiu-Ting Y., Haggerty, R. (2008) A Comparison of Two Probability Encoding Methods: Fixed Probability vs. Fixed Variable Values. Decision Analysis, Vol. 5, No. 4, 190-202.

[2] Abu Aarqob, O.A., Shawagfeh, N., Abu Ghneim, O.A. (2008) Functions Defined on Fuzzy Real Numbers According to Zadeh's Extension. International Mathematical Forum, Vol. 3, No. 16, 763-776.

[3] Alberts, C., Dorofee, A. (2002) Managing Information Security Risks: The OCTAVE Approach. Addison-Wesley: New York.

[4] Alberts, C., Dorofee, A. (2005) OCTAVE-s Method Implementation Guide Version 2.0. Canergie Mellon University: Pittsburgh.

[5] Bonissone, P.P. (1987) Selecting Uncertainty Calculi and Granularity: An Experiment in Trading-off Precision, Complexity. In Uncertainty in Artificial Intelligence, Amsterdam, 57-66.

[6] Bortolan, G., Degani, R. (1985) A Review of Some Methods for Ranking Subsets. Fuzzy Sets and Systems, Vol. 15, 1-19.

[7] Cabrera Silva, M., Vinagre Bachiller, J.M., Jabbour Padilla, P., Román Muñoz, F. (2010) Implantación de un Sistema de Gestión de la Seguridad de la Información y Certificación ISO 27001 en la Administración Pública. Tecnimap 2010. Subdirección General de Tecnologías de la Información Ministerio de Sanidad y Política Social. Gobierno de España.

[8] Calder, A., Watkins, S. (2008) IT Governance: A Managerís Guide to Data Security, ISO27001/ISO 27002. Kogan Page: London.

[9] Chen, S.M. (1985) Operations on Fuzzy Numbers with Function Principle. Tamkang Journal of Management Science, Vol. 6, 13-25. 
[10] Chen, S.M. (1996) New Methods for Subjective Mental Workload Assessment, Fuzzy Risk Analysis. Cybernetics Systems, Vol. 27, 449-472.

[11] Chen, S.J., Chen, S.M. (2003) Fuzzy Risk Analysis Based on Similarity Measures of Generalized Fuzzy Numbers. IEEE Transactions on Fuzzy Systems, Vol. 11, 45-56.

[12] Chen, S.J., Chen, S.M. (2007) Fuzzy Risk Analysis Based on the Ranking of Generalized Trapezoidal Fuzzy Numbers. Applied Intelligence, Vol. 26, 1-11.

[13] Chen, S.J., Hwang, C.L. (1992) Fuzzy Multiple Attribute Decision Making. SpringerVerlang: Berlin.

[14] CCTA Risk Analysis, Management Method (CRAMM), Version 5.0 (2003) Central Computing, Telecommunications Agency (CCTA): London.

[15] Cojazzi, G., Guida, G., Pinola, L., Sardella, R., Baroni, P. (1987) KEEJAM: a Knowledge Engineering Methodology for Expert Judgement Acquisition, Modelling in Probabilistic Safety Assessment. In Advances in Safety, Reliability, Vol. 1, 199-206. C. Guedes Soares, Pergamon.

[16] Dekkers, A., Aarts, E. (1991) Global Optimization, Simulated Anealing. Mathematical Programming, Vol. 50, No. 1-3, 367-393.

[17] Dokas, I.M., Nordlander, T.E., Wallace, R.J., (2007) Fuzzy Fault Tree Representation, Maintenance Based on Frames and Constraint Technologies: a Case Study. In: Proc. K-CAP'07 Workshop on Knowledge Capture, Constraint Programming, Whistler, British Columbia, Canada.

[18] Duarte, A., Pantrigo, J.J., Gallego, M. (2007) Metaheurísticas. Servicio de Publicaciones de la Universidad Rey Juan Carlos: Madrid.

[19] Font, J.J., Miralles, A., Sanchis M. (2012) On the Fuzzy Number Space with the Level Convergence Topology. Journal of Function Spaces, Applications, Vol. 2012, $1-11$.

[20] Hejazi, S.R., Doostparast, A., Hosseini, S.M. (2011) An Improved Fuzzy Risk Analysis Based on a New Similarity Measures of Generalized Fuzzy Numbers. Expert Systems with Applications, Vol. 38, 9179-9185. 
[21] Gil, M.A., López-Díaz, M., Ralescu, D. (2006) Overview on the Development of Fuzzy Random Variables. Fuzzy Sets and Systems, Vol. 157, 2546-2557.

[22] García Cascales, M.S. (2009) Métodos para la Comparación de Alternativas Mediante un Sistema de Ayuda a la Decisión (SAD) y Soft Computing. Tesis doctoral. Universidad Politécnica de Cartagena.

[23] Gomathi, N., Sivaraman, V.L. (2012) A Novel Similarity Measure Between Generalized Fuzzy Numbers. International Journal of Computer Theory and Engineering, Vol. 4, 448-450.

[24] Gómez Fernández, L., Álvarez A. (2012) Guía de Aplicación de la Norma UNE-ISO 27001 sobre Seguridad en Sistemas de Información para Pymes. AENOR ediciones: Madrid.

[25] Halliwell, J. (2007) Linguistic Probability Theory. PhD Thesis. University of Edinburgh.

[26] ISO/IEC 17799-2005 (2005) Information Tecnology — Security Techniques - Code of Practice for Information Security Management. Geneva, Switzerland.

[27] ISO/IEC 27001:2005 (2005) Information technology - Security Techniques — Information Security Management Systems - Requirements. Geneva, Switzerland.

[28] ISO/IEC 27000:2009 (2009) Information Security Management Systems - Overview, Vocabulary. Geneva, Switzerland.

[29] ISO/IEC 27003:2010 (2010) Information security management system implementation guidance. Geneva, Switzerland.

[30] ISO/IEC TR 13335-3:1998 (1998) Information Technology — Guidelines for the Management of IT Security - Part 3: Techniques for the Management of IT Security. Geneva, Switzerland.

[31] ISO/IEC 27005:2011 (2011) Information Technology - Security Techniques - Information Security Risk Management. Geneva, Switzerland.

[32] Jiménez-Martín, A., Vicente, E., Mateos, A. (2015) Selección de Salvaguardas en Gestión del Riesgo en Sistemas de la Información: Un Enfoque Borroso. Revista Ibérica de Sistemas y Tecnologías de la Información, Vol. 15, 83-100. 
[33] Kirkpatrick, S., Gelatt, C.D., Vecchi, Jr., M.P. (1983) Optimization by Simulated Annealing. Science, Vol. 220, No. 4598, 671-680.

[34] Kumar, A., Singh, P., Kaur, J. (2010) Generalized Simplex Algorithm to Solve Fuzzy Linear Programming Problems with Ranking of Generalized Fuzzy Numbers. Turkish Journal of Fuzzy Systems, Vol. 1, No. 2, 80-103.

[35] Kwakernaak, H. (1978) Fuzzy Random Variables. Part I: Definitions, Theorems. Information Science, Vol. 15, 1-29.

[36] Kwakernaak, H. (1979) Fuzzy Random Variables. Part II: Algorithms, Examples for the Discrete Case. Information Science, Vol. 17, 253-278.

[37] López Crespo, F., Amutio Gómez, M.A., Candau, J., Mañas, J.A. (2006a) Methodology for Information Systems Risk. Analysis and Management (MAGERIT version 2). Book I-The Method. Ministerio de Administraciones Públicas: Madrid.

[38] López Crespo, F., Amutio Gómez, M.A., Candau, J., Mañas, J.A. (2006b) Methodology for Information Systems Risk Analysis, Management (MAGERIT version 2). Book II-Catalogue of Elements. Ministerio de Administraciones Públicas: Madrid.

[39] López Crespo, F., Amutio Gómez, M.A., Candau, J., Mañas, J.A. (2006c) Methodology for Information Systems Risk Analysis, Management (MAGERIT version 2). Book III-The Techniques. Ministerio de Administraciones Públicas: Madrid.

[40] Martín del Brío, B., Sanz Molina. A. (2006) Redes Neuronales y Sistemas Borrosos. Ra-Ma: Madrid.

[41] Mehari (2010) - Risk Analysis, Treatment Guide. Club de la Sécurité de l' information Francais (CSIF): Paris.

[42] Merkhofer, M. (1987) Quantifying Judgemental Uncertainty: Methodology, Experiences, Insights. IEEE Transactions on Systems, Management and Cybernetics, Vol. 17, No. 5, 741-752.

[43] Morgan, M.G., Henrion, M. (1990) Uncertainty: A Guide to Dealing With Uncertainty in Quantitative Risk, Policy Analysis. Cambridge University Press: Cambridge. 
[44] Murakami, S., Maeda, S., Imamura, S. (1983) Fuzzy Decision Analysis on the Development of Centralized Regional Energy Control System. In Proc. IFAC Symposium on Fuzzy Information Knowledge Representation and Decision Analysis, 363-368.

[45] Stoneburner, G., Gougen, A. (2002) NIST 800-30 Risk Management. Guide for Information Technology Systems. National Institute of Standard, Technology: Gaithersburg.

[46] Syalim, A., Hori, Y., Sakurai, K. (2009) Comparison of Risk Analysis Methods: Mehari, Magerit, NIST800-30, Microsoft's Security Management Guide. In: Proc. Int. Conference disponibilidad, Reliability, Security, 726-735.

[47] Peña, R. (2006) De Euclides a Java. Historia de los Algoritmos y de los Lenguajes de Programación. NiVola: Madrid

[48] Ralescu, D.A. (1995) Fuzzy Probabilities and Their Applications to Statistical Inference, In: B. Bouchon-Meunier, R.R. Yager, L.A. Zadeh (eds.), Advances in Intelligent Computing-IPMU-94, Lecture Notes in Computer Science, Vol. 945, 217-222.

[49] Ríos, D., Ríos, S., Martín, J., Jiménez, A. (2008) Simulación. Métodos y Aplicaciones. Ra-Ma: Madrid.

[50] Ríos-Insua, S. (1988) Investigación Operativa. Centro de Estudios Ramón Areces: Madrid.

[51] Sagaya Roseline, S., Henry Amirtharaj, E.C. (2011) Generalized Fuzzy Modified Distribution Method for Generalized Fuzzy Transportation Problem. International Multidisciplinary Research Journal, Vol. 1, No. 10, 12-15.

[52] Saint-Germain, R. (2005) Information Security Management Best Practice Based on ISO/IEC 17799. The Information Management Journal, 60-67.

[53] Soo, K.J. (2000) How Much Is Enough? A Risk-Management Approach to Computer Security, Consortium for Research on Information Security and Policy (CRISP). Stanford University.

[54] Spetzler, C.S., Von Holstein. C.A. (1975) Probability Encoding in Decision Analysis. Management Science, Vol. 22, No. 3, 340-358. 
[55] Sridevi, B., Nadarajan, R. (2009) Fuzzy Similarity Measure for Generalized Fuzzy Numbers, International Journal Open Problems Computational Mathematics, Vol. 2, No. 2, 240-253.

[56] Tversky, A., Kahneman, D. (1974) Judgment under Uncertainty: Heuristics, Biases. Science, New Series, Vol. 185, No. 4157, 1124-1131.

[57] U.S. Nuclear Regulatory Commission (1990) Severe Accident Risks: An Assessment for Five U.S. Nuclear Power Plants. NRC Publications: New York.

[58] VV.AA. (2008) Expert Elicitation: Methodological Suggestions for its use in Environmental Health Impact Assessments. National Institute for Public Health, the Environment.

[59] VV.AA. (2011) Expert Elicitation Task Force White Paper. U.S. Environmental Protection Agency.

[60] Verdigay J.L., Vergara E.R. (2000) Fuzzy Termination Criteria in Knapsack Problem Algorithms. Mathware, Soft Computing, Vol. 7, 89-97.

[61] Vicente, E., Jiménez, A., Mateos, A. (2013a) A Fuzzy Approach to Risk Analysis in Information Systems. In: Proc. 2nd International Conference on Operations Research, Enterprise Systems, 130-133. Barcelona, Spain.

[62] Vicente, E., Mateos, A., Jiménez, A. (2013b) A New Similarity Function for Generalized Trapezoidal Fuzzy Numbers. In: Proc. 12th International Conference on Artificial Intelligence, Soft Computing, 400-411. Zakopane, Poland.

[63] Vicente, E., Jiménez, A., Mateos, A. (2013c) An interactive method of fuzzy probability elicitation in risk analysis. In: 4th International Conference on Risk Analysis, Crisis Response, 223-228. Istanbul, Turkey.

[64] Vicente, E., Mateos, A., Jiménez, A. (2013d) Análisis de Riesgos en los Sistemas de Información. In: Actas 8 a Conferencia Ibérica de Sistemas e Tecnologías de Informacao (CISTI 2013), 557-562. Lisbon, Portugal.

[65] Vicente, E., Jiménez, A., Mateos, A. (2013e) A Fuzzy Extension of MAGERIT Methodology for Risk Analysis in Information Systems. In: Proc. IADIS Information Systems Conference, 39-46. Lisbon, Portugal. 
[66] Vicente, E., Mateos, A., Jiménez-Martín, A. (2014a) A Fuzzy Approach based on Dynamic Programing, Metaheuristics for Selecting Safeguard for Risk Management for Information Systems. In: Proc. 3nd International Conference on Operations Research, Enterprise Systems, 35-45. Angers, Loire Valley, France.

[67] Vicente, E., Mateos, A., Jiménez-Martín, A. (2014b) Selection of Safeguards for Fuzzified Risk Management in Information Systems. In: New Perspectives in Information Systems, Technologies, Lecture Notes in Computer Science, 267-276. Springer, Switzerland.

[68] Vicente, E., Mateos, A., Jiménez-Martín, A. (2014c) Searching for a Consensus Similarity Function for Generalized Trapezoidal Fuzzy Numbers. In: Decision Making and Soft Computing. World Scientific Proc. Series on Computer Engineering and Information Science, 252-257.

[69] Vicente, E., Mateos, A., Jiménez-Martín, A. (2014d), Risk Analysis in Information Systems: a Fuzzification of the MAGERIT Methodology. Knowledge-Based Systems, Vol. 66, 1-12.

[70] Vicente, E., Mateos, A., Jiménez, A. (2014e) Elicitación de Probabilidades Difusas, In: Actas XVII Congreso Español sobre Tecnologías y Lógica Fuzzy, 33-38. Zaragoza, España.

[71] Vicente, E., Jiménez, A., Mateos, A. (2015a) Similarity Functions for Generalized Trapezoidal Fuzzy Numbers: An Improved Comparative Analysis. Journal of Intelligent, Fuzzy Systems, Vol. 28, 821-833.

[72] Vicente, E., Mateos, A., Jiménez-Martín, A. (2015b) Fuzzified Risk Management: Selection of Safeguards to Minimize the Maximum Risk. Computer Science and Information Systems, Vol. 12, No. 2, 567-585.

[73] Vicente, E., Mateos, A., Jiménez-Martín, A. (2015c) A Fuzzy Model for Selecting Safeguards to Reduce Risks in Information Systems. In: Operations Research and Enterprise Systems, Communications in Computer and Information Science. Springer, 63-78. 
[74] Wang, J., Hao, J. (2006) A New Version of 2-Tuple Fuzzy Linguistic Representation Model for Computing with Words. IEEE Transactions on Fuzzy Systems, Vol. 14, $435-445$.

[75] Wang, X., Kerre, E.E. (2001) Reasonable Properties for the Ordering of Fuzzy Quantities (I, II). Fuzzy Sets and Systems, Vol. 118, No. 3, 375-385.

[76] Wei, S.H., Chen, S.M. (2009) A New Approach for Fuzzy Risk Analysis Based on Similarity Measures of Generalized Fuzzy Numbers. Expert Systems with Applications, Vol. 36, 589-598.

[77] Wen, J., Fan, X., Duanmu, D., Yong, D. (2011) A Modified Similarity Measure of Generalized Fuzzy Numbers, Procedia Engineering, Vol. 15, 2773-2777.

[78] Xu, Z., Shang, S., Qian, W., Shu, W. (2010) A Method or Fuzzy Risk Analysis Based on the New Similarity of Trapezoidal Fuzzy Numbers. Expert Systems with Applications, Vol. 37, 1920-1927.

[79] Zadeh, L.A. (1965) Fuzzy Sets. Information Control, Vol. 8, 338-353.

[80] Zadeh, L.A. (1968) Probability Measures of Fuzzy Events. Journal of Mathematical Analysis, Applications, Vol. 23, 421-427.

[81] Zadeh, L.A. (1973) Outline of a New Approach to the Analysis of Complex Systems, Decision Processes. IEEE Transactions Systems Management and Cybernetics, Vol. $3,28-44$.

[82] Zadeh, L.A. (1975) The Concept of a Linguistic Variable, its Application to Approximate Reasoning. Part 1. Information Science, Vol. 8, 199-249.

[83] Zadeh, L.A. (1975) The Concept of a Linguistic Variable, its Application to Approximate Reasoning. Part 2. Information Science, Vol. 8, 301-353.

[84] Zadeh, L.A. (1975) The Concept of a Linguistic Variable, its Application to Approximate Reasoning. Part 3. Information Science, Vol. 9, 43-80.

[85] Zhu, L., Xu, R. (2012) Fuzzy Risk Analysis based on Similarity Measure of Generalized Fuzzy Numbers. In: Fuzzy Enginering and Operations Research. Springer: Berlin. 